\title{
Microbial interactions in the fish gut
}
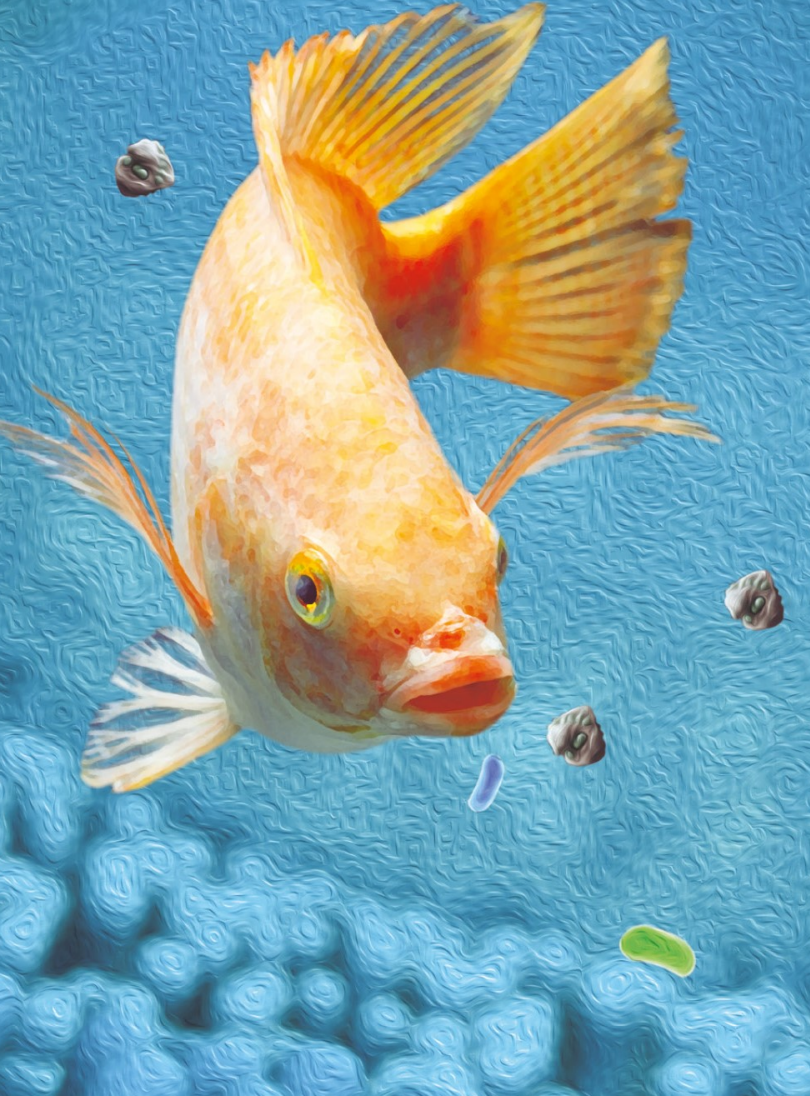

$$
\text { 6) Christios Giatsis }
$$





\section{Microbial interactions in the fish gut}

Christos Giatsis 


\section{Thesis committee}

\section{Promotor}

Prof. Dr Johan A.J. Verreth

Professor of Aquaculture and Fisheries

Wageningen University

\section{Co-promotors}

Dr Marc C.J. Verdegem

Associate professor, Aquaculture and Fisheries Group

Wageningen University

Dr Detmer Sipkema

Assistant professor, Laboratory of Microbiology

Wageningen University

\section{Other members}

Prof. Dr Jerry Wells, Wageningen University

Dr Kari J.K. Attramadal, NTNU, Trondheim, Norway

Prof. Dr Peter Bossier, Gent University, Belgium

Dr Walter J.J. Gerrits, Wageningen University

This research was conducted under the auspices of the Graduate School WIAS (Wageningen Institute of Animal Sciences). 


\section{Microbial interactions in the fish gut}

\section{Christos Giatsis}

\section{Thesis}

submitted in fulfillment of the requirements for the degree of doctor at Wageningen University

by the authority of the Rector Magnificus

Prof. Dr A.P.J. Mol,

in the presence of the

Thesis Committee appointed by the Academic Board

to be defended in public

on Friday 21 October 2016

at 4 p.m. in the Aula. 
Christos Giatsis

Microbial interactions in the fish gut 198 pages.

PhD thesis, Wageningen University, Wageningen, NL (2016)

With references, with summary in English

ISBN: 978-94-6257-877-7

DOI: $10.18174 / 387232$ 
Fiat justitia - ruat caelum -Ancient Roman proverb- 



\section{Contents}

Chapter 1

Introduction and thesis outline

\section{Chapter 2}

The colonization dynamics of the gut microbiota in tilapia larvae

\section{Chapter 3}

The impact of rearing environment on the development of gut microbiota in tilapia larvae

\section{Chapter 4}

Steering the gut microbiome of tilapia larvae with microbial diets

\section{Chapter 5}

Probiotic legacy effects on gut microbial assembly in tilapia larvae

Chapter 6

General discussion

References

Summary

Acknowledgements

About the author

List of publications

Training and Supervision Plan 



\title{
Chapter 1
}

\author{
Introduction and thesis outline
}

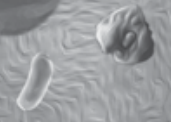

(b)

(2)

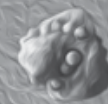

\section{(ic)}




\subsection{Bottlenecks in aquaculture}

Aquaculture is growing more rapidly than all other animal food producing sectors, with an average yearly growth rate of more than $6 \%$ over the last 20 years (FAO, 2014). Over the past years there has been a considerable progress in aquaculture mainly of empirical character, but partly also of fundamental nature. This progress together with the increasing demand of seafood has made aquaculture a profitable business.

Despite significant advances, the aquaculture still faces major bottlenecks related to suboptimal growth, inexplicable mortalities and diseases as well as malformations and variable quality of juveniles (Attramadal et al., 2012). Attempts in overcoming the aforementioned difficulties focused mainly on broodstock management and breeding, optimization of water quality and on species dietary requirements. However, fish performance (health and growth) still varies considerably between identically reared individuals, even when from the same sibling group. Fish-microbe interactions have been suggested to be a key factor in explaining this lack of reproducibility (Vadstein et al., 2013).

Gut microbiota influences a wide range of biological processes in humans (Rawls, 2007; Sekirov et al., 2010), domesticated terrestrial animals (Chaucheyras-Durand and Durand, 2010; McFall-Ngai et al., 2013) and fish (Nayak, 2010b; Sullam et al., 2012). Despite the significant contribution of several studies on fish gut microbiota, our understanding on the functional significance of microbial fluctuations lags well behind that of terrestrial vertebrates.

\subsection{Fish in a microbial world}

The nutrient rich environment of aquaculture tanks and ponds favors the proliferation of microbes. Exposed body surfaces of fish, including the gut, are quickly colonized right after hatching mostly due to the influence of water communities (Reitan et al., 1998; Verschuere et al., 2000). Feed microbiota becomes important later mostly from the time that active feeding occurs (Hansen and Olafsen, 1999; Ringo and Birkbeck, 1999). This dynamic community is subjected to changes related to the developmental stage of the host and constantly adapts to shifts in the nutritional and environmental situation (Nayak, 2010b; Romero and Navarrete, 2006; Navarrete et al., 2012; Li et al., 2012b). Fish gut microbiota vary within species due to factors such as season, salinity, $\mathrm{pH}$, temperature, trophic level and feed (Nayak, 2010b; Sullam et al., 2012).

Although water and feed are the main sources of microbes, fish and their gut bacteria seem to have more complex relationships than one would expect from a simple reflection of the free-living microbial community in the environment (Sullam et al., 2012). For the ingested microbes, the gut provides different (micro) habitat niches leading to the 
establishment of a very complex microbial community upon which the host has a great influence (Sommer and Bäckhed, 2013; Hansen and Olafsen, 1999).

\subsection{Host-microbe interactions}

Fish microbial communities are strongly influenced by, but also influence, their hosts. (Sullam et al., 2012). The beneficial role of the intestinal microbiota in fish is recently being explored, suggesting that microbiota stimulates nutrient metabolism, innate immune response and epithelial proliferation (Roeselers et al., 2011). Additionally, gut microbiota constrains the colonization of infection agents and, by interacting with the host, mediate the development, maintenance and effective functionality of the intestinal mucosa (Dimitroglou et al., 2011).

The majority of the ingested microbes are only transient in the gut (Sugita et al., 1996). For ingested bacteria to proliferate and persist as "resident" microbiota, they must be capable to adapt to the environmental conditions inside the gut, such as nutrient availability, $\mathrm{pH}$ and digestive enzymes (Hansen and Olafsen, 1999). Host dependent selective pressures impacting gut microbiota in fish, are mostly attributed to gut habitat (ecology) and host genotype (Yan et al., 2012; Rawls et al., 2006; Navarrete et al., 2012).

Nevertheless, insights in the roles of microbial communities and the factors that drive their diversity remain limited. Apart from a few pathogens, host-microbe interactions in fish are still poorly understood and unravelling composite relationships within the fish gut remains challenging mostly due to the ecological and functional complexity of this ecosystem.

\subsection{Microbial community management}

Lack of understanding on the driving forces governing microbial colonization in the gut makes it difficult to develop applications or to interfere in the process of microbial community assembly. The following strategies have so far mainly been applied for microbial control management in aquaculture.

Reduction of microbial load: This strategy mostly aims at the reduction of microbes in a non-selective manner. Such methods include the use of antibiotics or disinfectants either by administrating them directly to the host or by treating the surrounding environment. Besides the risk of promoting antibiotic resistant microbes, the main drawback of these methods is the proliferation of opportunistic microbes due to the large and non-selective reduction of microbial load (Hess-Erga et al., 2010). 
Reduction of organic load: This is a strategy for semi-selective microbial reduction in the sense of a non-specific targeting of particular taxa. However, different microbes use different substrates and the removal of easily degradable organic matter selects against opportunistic heterotrophic bacteria. The easily degradable organic matter is removed from the water, thus indirectly decreasing the overall bacterial load in the system (Attramadal et al., 2012). However, rapid fluctuations of the organic load in the system should be avoided due to the unpredictable proliferation of opportunistic species.

Use of pro and prebiotics: The use of live microorganisms (probiotics) that confer a health benefit to the host appears promising in aquaculture (Pérez-Sánchez et al., 2014). Probiotics reduce infections caused by bacterial pathogens (Gatesoupe, 1999; Martínez Cruz et al., 2012) and are successfully used as immunostimulants (Cain and Swan, 2010; Lepage et al., 2012; Song et al., 2014) and growth promoters in fish and shrimp (Pérez-Zánchez et al., 2013; Merrifield et al., 2010). However, these strains seem to persist only temporarily in the gut after supplementation is stopped. The use of prebiotics as a nutritional substrate for the selective enrichment of specific probiotic species could facilitate the establishment/ selection of beneficial bacteria.

Apart from the use of antibiotics and the implementation of hygiene protocols, microbial management techniques have limited fundamental scientific backing and there has been a rush to empirical solutions bypassing the need for more fundamental knowledge on host-microbial interactions in aquaculture species. Establishing a desired gut microbiota in fish larvae and assuring proper functionality is not straight forward. First colonization in particular, can be assumed to have long-term impact for larval health. This encourages the development of microbial management strategies to steer first colonization. Developing the proper tools for microbial assembly management implies gaining insight into the ecological mechanisms and processes that govern it, which is the main focus of this thesis.

\subsection{Thesis aim and outline}

All experimental chapters of this thesis focused on the early developmental stages (i.e. from first feeding to 6 weeks old) of Nile tilapia Oreochromis niloticus (Linnaeus) larvae. Tilapia larvae were chosen mostly because: a) early life stages are crucial for fish development and thus the base for a successful production cycle, b) tilapia generally feeds in shallow waters grazing on sediments, which creates a direct contact of the fish with a large and diverse microbial load and c) the impact of free-living bacteria on gut microbiota is more pronounced during early ontogenetic stages when the fish gut is not yet fully developed and the immune system is still immature. 
This thesis starts from the concept that the fish gut microbial communities (MC) are influenced by the host itself and by the free-living MC of host's habitat. However, colonization of the fish gut is partially a stochastic process, with successful bacteria being those that are present at the right moment and place. Stochastic phenomena have been proposed as a cause of high inter-individual variation in gut microbiota (Fjellheim et al., 2007) which could in turn be associated with highly variable quality and health of reared animals.

To comprehend the full range of diversity of gut microbiota, it is very important to characterize and quantify the inter- and intra-individual variation in space and time. In fact, the characterization of the variation between identically reared individuals can serve as baseline to determine the contribution of stochastic factors to the overall variation. For this reason we initially characterized the variation between identically reared individuals to determine the contribution of stochastic factors to the overall variation. In chapter $\mathbf{2}$ we quantified the spatio-temporal variation of water and gut microbiota of Nile tilapia larvae, reared in different production systems i.e. recirculating or active suspension systems (RAS vs. AS). The location effects on gut microbiota of newly hatched larvae were compared to individuals reared within the same or between replicate tanks, and between replicate and different systems for a period of 6 weeks.

Due to rapid shifts in the microbial community composition during early ontogenetic stages, it is difficult to relate changes in gut microbiota to alterations of a single environmental factor. By understanding the factors underlying the successful colonization of ingested microbes and the community assembly we could increase predictability and repeatability of gut microbiota interventions. Regarding the relative importance of water and feed microbiota in determining gut microbial composition, outcomes from experimental studies are controversial. There seems to be a general acceptance that diet is a major contributor of microbes for the gut of fish larvae (Nayak, 2010b; Reid et al., 2009), but this hypothesis is not supported across studies (Bakke et al., 2013). In order to provide first insights into the forces that structure fish gut communities and illustrate $M C$ affinities to symbiotic and free-living communities, the similarity among gut bacteria of fish and the free-living bacteria present in their environments (i.e. water) was investigated. Chapter $\mathbf{3}$ evaluates the relative contribution of water and feed microbiota to gut microbiota composition of larvae. Therefore, the composition of gut microbiota of tilapia larvae reared in either RAS or AS systems from the moment of first feeding was characterized at different time points. Better understanding of how microbiota in water, feed and gut interact will help improving the design, microbial management and nutrient cycling of fish rearing systems.

In chapters 2 and 3, input of bacteria via the feed was the common denominator (all treatments received the same commercial feed) whereas the impact of water MC on gut communities was evaluated by testing the effect of different rearing systems. In chapter 4 the opposite scenario was investigated i.e. all larvae shared the same water (reared in 
the same system), but were fed with different diets. We explored the potentials of steering gut communities during early life stages of tilapia via microbial manipulations of feed communities. We hypothesized that if gut microbial composition is strongly shaped by selective pressures in the gut, then similar gut microbial communities should develop regardless of the dietary treatment. Contrary, if the gut microbial community is mostly shaped by the composition of the microbial community present in the diet, this will result in a significantly different gut microbiota between treatments. To test our hypothesis fish larvae were fed four diets with microbiota derived from aerobic, methanogenic or denitrifying sludge reactors. All individuals were reared in the same system to avoid variation between treatments due to differences in water microbiota. We performed 16S rRNA gene sequencebased comparisons between gut microbial communities from different treatments and we associated them with the ones present in feed and water.

Probiotics have been widely applied in aquaculture for many years. However, probiotic strains often colonize the gut only transiently and quickly fall below detection limits (Gatesoupe, 1999; Grześkowiak et al., 2012). Currently, delivery of probiotics through feed in larval stages is the most common strategy for management of larval gut microbiota in aquaculture (Nayak, 2010a). The administration of the probiotic strain on early ontogenetic stage, when the gut microbiota still develops, could enhance gut-colonization of the probiotic itself and may therefore lead to the development of distinct gut communities. In chapter $\mathbf{5}$ we tested whether an early contact of larvae with bacteria has a long lasting effect on gut microbial community composition on later life stages, i.e. an imprinting effect. We hypothesized that an early contact of fish larvae with the probiotic strain would have an impact on shaping gut microbiota even after the administration of the probiotic discontinues. To do this, we constrained the initial contact with microbes from the environment by producing both axenic hosts and hosts with a known microbial community (addition of a single strain probiotic). Subsequently, the imprinting hypothesis was tested by exposing the two host types to similar husbandry conditions.

Finally, chapter 6 discusses the main results from all experimental chapters in the context of the existing literature, and overall conclusions and practical implications are presented. Furthermore, a meta-analysis of 16S rRNA gene sequence data from 12 different studies on gut microbiota of different fish species was performed. The meta-analysis incorporated datasets from the three studies included in this thesis, two unpublished datasets from our previous studies on tilapia larvae and seven publically available gut microbiome datasets from studies performed in trout, carp, zebrafish and tilapia. By examining the phylogenetic similarity among gut bacteria of the same and different fish species from different habitats, diets, facilities and developmental stage, we investigated the factors that primarily shape these communities. The role of host selectivity over environmental impact on gut communities was investigated by comparing phylogenetic distance between gut bacteria of the different fish species and studies. 


\section{Chapter 2}

The colonization dynamics of the gut microbiota in tilapia larvae

i)
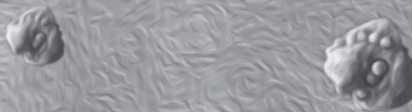

This chapter has been published as:

Giatsis C, Sipkema D, Smidt H, Verreth J, Verdegem M (2014) The Colonization Dynamics of the Gut Microbiota in Tilapia Larvae. PLoS ONE 9(7): e103641. doi:10.1371/journal.pone.0103641 
The gut microbiota of fish larvae evolves fast towards a complex community. Both host and environment affect the development of the gut microbiota; however, the relative importance of both is poorly understood. Determining specific changes in gut microbial populations in response to a change in an environmental factor is very complicated. Interactions between factors are difficult to separate and any response could be masked due to high interindividual variation even for individuals that share a common environment. In this study we characterized and quantified the spatio-temporal variation in the gut microbiota of tilapia larvae, reared in recirculating aquaculture systems (RAS) or active suspension tanks (AS). Our results showed that variation in gut microbiota between replicate tanks was not significantly higher than within tank variation, suggesting that there is no tank effect on water and gut microbiota. However, when individuals were reared in replicate RAS, gut microbiota differed significantly. The highest variation was observed between individuals reared in different types of system (RAS vs. AS). Our data suggest that under experimental conditions in which the roles of deterministic and stochastic factors have not been precisely determined, compositional replication of the microbial communities of an ecosystem is not predictable. 


\subsection{Introduction}

The gut of fish harbors a diverse microbial community. It provides niches for adherence, colonization and proliferation of mutualistic, benign commensal and pathogenic microbial species that affect many physiological and immunological functions of the host (Cahill, 1990; Gómez and Balcázar, 2008; Hansen and Olafsen, 1999). The microbial community in the gut changes with the developmental stage of the host and constantly adapts to the nutritional and environmental situation (Nayak, 2010b; Romero and Navarrete, 2006; Navarrete et al., 2012; Li et al., 2012b). Impacts on fish gut microbiota are more pronounced during early ontogenetic stages when the fish gut is not yet fully developed and the immune system is immature (Gatesoupe, 1999).

However, due to high inter-individual variation between fish and rapid changes in the microbial community composition during early life stages, it is difficult to relate changes in gut microbiota to alterations of a single factor. It has been suggested that inter-individual variation in gut microbial community composition both in humans (Walker et al., 2011) and animals (Haenen et al., 2013) might mask treatment effects. High individual variation was suggested as the reason for not detecting differences in gut microbiota in Atlantic salmon (Salmo salar) fed with different diets (Ringø et al., 2008). High inter-individual variation in quantity, diversity and richness of gut bacteria was also observed between individuals from the same tank in Bluefin tuna (Gatesoupe and Covès, 2012) as well as in cod larvae (Fjellheim et al., 2011).

Inter-individual variation in gut microbiota between individuals reared under the same conditions can be partially explained by stochastic processes (Fjellheim et al., 2007). However, "stochastic variation" cannot just be considered as "noise". To comprehend the full range of genetic and metabolic diversity of gut microbiota, it is very important to characterize and quantify the inter- and intra-individual variation in space and time. In fact, the characterization of the variation between identically reared individuals can serve as baseline to determine the contribution of stochastic factors to the overall variation.

In this study we characterized and quantified the spatio-temporal variation of water and gut microbiota of Nile tilapia larvae, reared for six weeks in two replicate Recirculating aquaculture systems (RAS). The location effects on larvae gut microbiota were compared for individuals reared within the same or between replicate tanks, and between replicate RAS systems. To determine the generality of any pattern observed in the RAS, and to avoid any affinity of the results with the specific habitat (RAS), temporal and replication effects were also studied in replicate active suspension (AS) systems also known as zeroexchange activated sludge systems or biofloc systems (Crab et al., 2012). 


\subsection{Materials and methods}

\subsubsection{Ethics Statement}

The experiment was approved by the Ethical Commission for Animal Experiments of Wageningen University (Project Name: Promicrobe; Registration code: 2009055d).

\subsubsection{Experimental animals and set up}

Three to four days old fertilized Nile tilapia eggs, obtained from TilAqua International (Velden, the Netherlands) were incubated at $27^{\circ} \mathrm{C}$. Two different culture systems were used to rear the newly hatched larvae: a recirculating aquaculture system (RAS) with two replicates ( $\mathrm{Ra}$ and $\mathrm{Rb}$ ), and an active suspension (AS) system with five replicates (AS 1-5). Each RAS contained five 20-L tanks and the tanks were connected to the same water purification unit. The two replicate RAS systems were not connected to each other. The five 120-L AS tanks were independent units and they did not share the same water. Initially, all systems were filled with water from the same source. In addition, before the start of the experiment, water and filter materials from the two RAS were mixed. Water of the five AS systems was treated similarly. The larvae were incubated together in a common tank before stocking. In each tank, 100 randomly selected swim-up larvae (7 days post fertilization) were introduced before the first feed application. Feeding started 9 days post fertilization (referred to as day 0; D00) and was continued for 42 days. Each day, larvae were fed with $0.5 \mathrm{~mm}$ commercial starter tilapia diet (F-0.5 GR Pro Aqua Brut - Trouw Nutrition, France) until apparent satiation for maximum 30 minutes at 9.00, 12.30 and 16.00 hours. The same type of feed, originating from a common batch, was used throughout the 42 day experimental period. Feed pellets were introduced slowly while observing feeding behavior, and administration stopped when it took more than 15 seconds before fishes reacted to newly fed pellets. Just before the first feeding, water and gut samples were collected, to determine the "initial" microbiota. Other samples were taken before the first daily feeding on day 07, 14, 28 and 42 (Figure S2.1).

Water physicochemical characteristics were maintained at safe levels for Nile tilapia larvae (pH 6.6 - 8.5, temperature $26-28^{\circ} \mathrm{C}, \mathrm{NH}_{3}-\mathrm{N}<0.2 \mathrm{mg} \mathrm{L}^{-1}, \mathrm{NO}_{2}-\mathrm{N}<1 \mathrm{mg} \mathrm{L}^{-1}$ and DO $>5 \mathrm{mg} \mathrm{L}^{-1}$ ). The photoperiod was set to 12 hours light -12 hours dark. During the experiment both RAS and AS operated as fully closed systems.

\subsubsection{Collection of gut and water samples}

On each sampling day ten larvae per tank were collected for microbial community analysis. The larvae were euthanized with $0.6 \mathrm{~g} \mathrm{~L}^{-1}$ Tricaine Methanesulfonate (TMS, 
Crescent Research Chemicals, Phoenix, Arizona, USA), buffered with $0.12 \mathrm{~g} \mathrm{~L}^{-1}$ sodium bicarbonate in water originating from the corresponding rearing tank. Subsequently, larvae were rinsed with $70 \%$ ethanol and sterile water before dissecting out aseptically the gut under a dissection microscope. Whole gut samples were flash frozen in liquid nitrogen and stored individually at $-80^{\circ} \mathrm{C}$ until subsequent analyses.

All tools and dissecting surfaces were disinfected with chloramine-T (Halamid-d, Veip BV, The Netherlands) and 70\% ethanol between dissections. In addition, the tools were always held in a propane gas flame before use.

From each tank, $250 \mathrm{~mL}$ water samples were collected at the same time of gut sampling. The water was filtered through $0.45 \mu \mathrm{m}$ (type HAWP) and $0.22 \mu \mathrm{m}$ (type GTTP) membrane filters (Millipore - Isopore) using a vacuum apparatus.

The microbiota in the water and gut was analyzed using denaturing gradient gel electrophoresis (DGGE) of PCR-amplified 16S ribosomal RNA (rRNA) gene fragments. One water and 3 gut samples were taken from each of the 15 tanks and analyzed by using PCRDGGE on days $0,7,14,28$ and 42. In addition, 1 water and 3 gut samples were taken from 2 replicate tanks of each RAS and from 2 AS tanks. Those samples were analyzed by 454 pyrosequencing of partial 16S rRNA genes on days 7 and 42. Samples that were analyzed with 454 pyrosequencing were a subset of the sample set that was analyzed with PCRDGGE.

\subsubsection{Genomic DNA isolation}

DNA was extracted from larval gut samples using the DNeasy Blood \& Tissue Kit (Qiagen, Venlo, Netherlands) according to the manufacturer's protocol with the following modifications: The gut samples were added to $180 \mu \mathrm{L}$ enzymatic lysis buffer and incubated at $37^{\circ} \mathrm{C}$ for 1 hour. Furthermore, $40 \mu \mathrm{L}$ proteinase $\mathrm{K}$ and $180 \mu \mathrm{L}$ ATL buffer were added to improve cell lysis, and the mix was incubated for $1.5 \mathrm{~h}$ at $55^{\circ} \mathrm{C}$. Cell-lysis was further optimized by performing an additional step in which gut tissue was homogenized in $200 \mathrm{~mL}$ AL buffer (Qiagen) with the aid of a custom bead mix (4 glass beads 2-3 $\mathrm{mm}, 0.5 \mathrm{~g}$ zirconia beads 0,1 mm) (MO-BIO Carlsbad, CA USA) and using the FastPrep instrument (QBioGene, Irvine, CA, USA) for $1 \mathrm{~min}$ at 6,000 rpm. The samples were eluted twice in $50 \mu \mathrm{L} A E$ buffer. DNA concentration was measured with a NanoDrop ND-1000 spectrophotometer (NanoDrop ${ }^{\circledR}$ Technologies, Wilmington, DE), and DNA samples were stored at $-20^{\circ} \mathrm{C}$ until use.

For DNA extraction from water samples, the FastDNA SPIN kit for soil (MP Biomedicals, Ohio, USA) was used. The DNA was extracted from water membrane filters. Briefly, homogenization was achieved by addition of $978 \mu \mathrm{L}$ sodium phosphate and $122 \mu \mathrm{L}$ MT buffer, and the cell lysis in the lysing matrix was enhanced by a bead beating step of 40 
$\mathrm{s}$ at $6000 \mathrm{rpm}$. DNA purification was achieved by addition of $1 \mathrm{~mL}$ silica binding matrix and $500 \mu \mathrm{L}$ SEWS-M (salt ethanol wash) followed by centrifugation at $14,000 \mathrm{~g}$ for $5 \mathrm{~min}$. The DNA was eluted by the addition of $50 \mu \mathrm{L}$ DES (DNA elution solution ultra-pure water) and incubated at room temperature for $5 \mathrm{~min}$. Subsequently, the DNA was collected by centrifugation at $14,000 \mathrm{~g}$ for $5 \mathrm{~min}$. For more details see instructions given by the manufacturer.

\subsubsection{PCR-DGGE analysis}

Target fragments of the bacterial 16S ribosomal RNA gene were amplified from the extracted DNA by PCR by using the following cycling conditions: $95^{\circ} \mathrm{C}$ for $2 \mathrm{~min}$, followed by 35 cycles consisting of $95^{\circ} \mathrm{C}$ for $30 \mathrm{~s}, 53^{\circ} \mathrm{C}$ for $40 \mathrm{~s}$ and $72^{\circ} \mathrm{C}$ for $1 \mathrm{~min}$ and then a final 5 min extension step at $72^{\circ} \mathrm{C}$. Samples were cooled to $4^{\circ} \mathrm{C}$. PCR for DGGE was performed by using primers L1401-R (5'-CGGTGTGTACAAGACCC-3') and U968-F (5'-CGCCCGGGGCGCGC CCCGG GCGGGGCGGGGGCACGGGGGGAACGCGAAGAACCTTAC-3') fitted with a GCclamp (Nubel et al., 1996). The PCR reaction mixture consisted of Phusion HF buffer, $0.2 \mu \mathrm{M}$ of each primer, $200 \mu \mathrm{M}$ of each dNTP, and 1 unit of Phusion Hot Start II High Fidelity Polymerase. To the $50 \mu \mathrm{L}$ reactions $20-50 \mathrm{ng}$ of DNA was added. Five $\mu \mathrm{L}$ of all PCR products were visualized by gel electrophoresis using $1 \%$ agarose gel with ethidium bromide to check the quality. DGGE analysis of PCR amplicons was performed as described previously (Muyzer et al., 1993) using the DCode system (Bio-Rad Laboratories, Hercules, CA). Polyacrylamide gels consisted of $8 \%$ (vol/vol) polyacrylamide (37.5:1 acrylamide-bisacrylamide) in $0.5 x$ Tris-acetate-EDTA. A denaturing acrylamide containing $7 \mathrm{M}$ urea and $40 \%$ formamide was defined as $100 \%$. The gels were poured from the top by using a gradient maker (Econopump; Bio-Rad, La Jolla, CA) and pumping the solution at a speed of $4.5 \mathrm{~mL} \mathrm{~min}^{-1}$. A gradient from 30 to $60 \%$ was used for the separation of the PCR amplicons. Electrophoresis was performed for $16 \mathrm{~h}$ at $85 \mathrm{~V}$ in a $0.5 x$ Tris-acetate-EDTA buffer at a constant temperature of $60^{\circ} \mathrm{C}$. Subsequently, gels were stained with $\mathrm{AgNO}_{3}$ according to the method described by Sanguinetti et al. (Sanguinetti et al., 1994).

\subsubsection{Pyrosequencing}

For more detailed 16S rRNA gene-based microbial composition profiling, barcoded amplicons from the V1-V2 region of 16S rRNA genes were generated by PCR using the 27F-DegS primer (Van den Bogert et al., 2011) that was appended with the titanium sequencing adaptor $A$ and an 8 nucleotide sample-specific barcode (Hamady et al., 2008) at the $5^{\prime}$ end. As a reverse primer, an equimolar mix of two primers $338 \mathrm{R} \mathrm{I}$ and II (van den Bogert et al., 2013) was used that carried the titanium adaptor $B$ at the $5^{\prime}$ end. Extracted DNA was diluted to a concentration of $20 \mathrm{ng} \mathrm{LL}^{-1}$ based on Nanodrop readings. PCR was performed using a GS0001 thermocycler (Gene Technologies, Braintree, United Kingdom). 
The PCR mix (100 $\mu \mathrm{L}$ final volume) contained $20 \mu \mathrm{L}$ of $5 \times$ HF buffer (Finnzymes, Vantaa, Finland), $2 \mu \mathrm{L} 10 \mathrm{mM}$ (each nucleotide) PCR-grade Nucleotide Mix (Roche Diagnostic $\mathrm{GmbH}$, Mannheim, Germany), $1 \mu \mathrm{L}$ of Phusion hot start II High-Fidelity DNA polymerase $(2 \mathrm{U} / \mu \mathrm{L})$ (Finnzymes), $500 \mathrm{nM}$ of the reverse primer mix and the forward primer (Biolegio BV, Nijmegen, The Netherlands); $2 \mu \mathrm{L}$ (i.e. $40 \mathrm{ng}$ ) template DNA and $65 \mu \mathrm{L}$ nuclease free water. PCR was performed under the following conditions: $98^{\circ} \mathrm{C}$ for $30 \mathrm{~s}$ to activate the polymerase, followed by 30 cycles consisting of denaturation at $98^{\circ} \mathrm{C}$ for $10 \mathrm{~s}$, annealing at $56^{\circ} \mathrm{C}$ for $20 \mathrm{~s}$, and elongation at $72^{\circ} \mathrm{C}$ for $20 \mathrm{~s}$, and a final extension at $72^{\circ} \mathrm{C}$ for $10 \mathrm{~min}$. Twenty $\mu \mathrm{L}$ of the PCR products were analyzed by $1 \%(\mathrm{w} / \mathrm{v})$ agarose gel electrophoresis in the presence of $1 \times S_{S B R}{ }^{\oplus}$ Safe (Invitrogen, Carlsbad, CA, USA) and purified from gel using the High Pure PCR Cleanup Micro Kit (Roche Diagnostics) according to manufacturer's instructions. DNA concentrations of gel-purified amplicons were measured by a Nanodrop ${ }^{\circledR}$ ND-1000 spectrophotometer, and purified PCR products were mixed in equimolar amounts, run again on an agarose gel and subsequently excised and purified using a DNA gel extraction kit (Milipore, Billerica, MA, USA). Nucleotide sequences were generated by pyrosequencing using an FLX genome sequencer in combination with titanium chemistry (GATC-Biotech, Konstanz, Germany). Pyrosequencing data were deposited at the European Bioinformatics Institute in the sequence read archive under study accession number PRJEB4462 and sample accession numbers ERS343984 - ERS344037.

The 454 pyrosequencing analysis was paired to the DGGE data by using samples collected from 2 replicate tanks on day 07 and 42, for the 2 RAS and 2 AS. We used both complementary methods for the characterization of spatiotemporal variation in the microbial communities in order to evaluate whether the outcome was consistent and comparable between methods, allowing for more general statements regarding the consequences for study design. Although pyrosequencing provided also useful direct sequence information with respect to the composition and ecology of the microbial communities in the samples, this was beyond the scope of this study and will be addressed in a separate paper.

\subsubsection{Normalization between DGGE gels}

On every DGGE gel a standard reference marker consisting of an amplicon mix of 10 different cloned bacterial 16S rRNA genes was included at 3 different positions, for digital gel normalization. These 10 fragments of the reference marker produced a known distinctive pattern defined by the position of the bands. The designation of the intergel band classes was based upon their relative position on the profile compared with the standard reference used, as described above. An overall comparison of the reference markers between all gels showed that all markers from the 15 gels clustered together, with a similarity higher than $95 \%$ and regardless of the gels that they belonged to, 
indicating that a valid comparison in community fingerprints was possible also between multiple gels. Using a standard reference marker to allow intra and inter-gel comparisons, has been suggested elsewhere (Joossens et al., 2011; Goodhead et al., 2013; Machiels et al., 2013; Thompson, 2014; Ercolini, 2004; Kowalchuk et al., 2006; Vanhoutte et al., 2004; Li et al., 2014b; Temmerman and Scheirlinck, 2003; Tourlomousis et al., 2010; Muyzer and Smalla, 1998). In addition to that, the inter-gel variation among profiles was tested at the beginning of the DGGE analysis. To this end, randomly selected samples from 2 or 3 different gels were selected and re-loaded into a single gel. DGGE gels (Figures S2.2, S2.3 and S2.4) showed that samples were clearly grouped based upon their band pattern and not upon the gel they belonged to, allowing for a fair inter-gel comparison.

\subsubsection{Data handling and statistical analysis}

DGGE patterns were analyzed with Bionumerics software 5.1 (Applied Maths, St-MartensLatem, Belgium) following the manufacturer's instructions. The patterns were normalized and individual bands were initially marked automatically (5\% minimum profiling), followed by visual inspection and manual correction whenever necessary. For automatic band matching the position tolerance of the fingerprints was set to $1 \%$ (percentage of the pattern length) maximum shift between two bands. Optimization for the best possible matching was set to a maximum allowable shift of $0.5 \%$. The band-classes were arbitrarily generated in a global alignment of all entries (DGGE lanes) of combined DGGE gels, by tracing common bands across different profiles. The designation of the band-classes was based on their position in the profile compared with the reference marker used as a normalization standard, to ensure gel-to-gel comparability. The bands were furthermore inspected manually for consistency. As measure of relative abundance, relative intensity of each band within individual DGGE profiles was used. Subsequently, data were square root transformed to decrease the importance of the most dominant bands in the subsequent analysis (Legendre and Gallagher, 2001).

Pyrosequencing data were analyzed using the QIIME 1.5.0 pipeline (Caporaso et al., 2010), and quality filtering (de-noising) was performed as follows. Low quality sequences were removed using default parameters (i. reads with fewer than 200 or more than 1000 nucleotides; ii. reads with more than 6 ambiguous nucleotides, homopolymer runs exceeding 6 bases, reads with missing quality scores and reads with a mean quality score lower than 25 ; iii. reads with mismatches in the primer sequence), and operational taxonomic units (OTUs) were identified at the $97 \%$ identity level. Representative sequences from the OTUs were aligned using PyNAST (DeSantis et al., 2006). The taxonomic affiliation of each OTU was determined using the RDP Classifier at a confidence threshold of $80 \%$ against the 12_10 Greengenes core set (Wang et al., 2007). Possible chimeric OTUs were identified using QIIME's ChimeraSlayer and removed from the initially generated OTU list, producing a final set of non-chimeric OTUs. 
For the DGGE data, there were five possible factors in the experimental design: "origin" (two levels; gut and water; fixed), "date" (five levels; day 0, 7, 14, 28, 42; fixed), "system type" (two levels; RAS and AS; fixed), "replicate system" (2 RAS or 5 AS; nested in system type: random) and "tank" (five levels, tank 1, 2, 3, 4 and 5, nested in replicate system: random). Because of the highly skewed distribution of bacterial species and the large number of zeros contributed by rare species, the assumption of multivariate normal distribution was unrealistic. For that reason a permutation-based multivariate ANOVA (PERMANOVA) was used to analyze the data set (Anderson, 2001). This method allows multivariate data to be analyzed on the basis of any distance or dissimilarity measure. The distance matrix was based on Bray Curtis dissimilarity (Bray and Curtis, 1957) due to its desirable properties when compared to other distance measures for analyzing environmental data. For example, the Bray Curtis coefficient does not increase the similarity between two samples when a common species absence occurs (Clarke, 1993) which is a very useful property when analyzing biological assemblage data with many zeros. For each term in the analysis, 9999 permutations of raw data units were performed to calculate P values, and when there were not enough possible permutations a Monte Carlo sample was drawn from the theoretical asymptotic permutation distribution (Anderson and Robinson, 2003).

In addition to PERMANOVA, analysis of similarities (ANOSIM) was used to give an insight into the degree of separation between the tested groups of samples. ANOSIM tests the null hypothesis that the average rank similarity between samples within a group is the same as the average rank similarity between samples belonging to different groups. The analysis produces an $\mathrm{R}$ statistic that generally ranges from 0 to 1 (Chapman and Underwood, 1999). An R of 1 indicates complete separation whereas an R of 0 indicates that the null hypothesis is true. The statistical significance of $R$ statistic is assessed by random permutations of the group membership to obtain the empirical distribution of $R$ under the null-model and is free of any assumption of normality (Clarke, 1993).

Although neither PERMANOVA nor ANOSIM explicitly assume common variances among groups, they are both sensitive to differences in multivariate dispersion. To test the hypothesis of equal within group dispersion (for both methods) PERMDISP analysis as a multivariate non-Euclidean equivalent to traditional Levene's test was used (Anderson, 2006). The analysis was used for two reasons: i. as a complementary test to avoid any kind of misinterpretation of the outcome of the two previous methods mostly due to type II error, and ii. to give insight of within and between groups variation. Homogeneity of dispersion among groups was calculated as an average distance $( \pm \mathrm{SE})$ of group members (samples) from the group's centroid. PERMDISP was used to test the null hypothesis of no difference between groups dispersion. Significant effects on group dispersion were tested for "tank", "replicate system", "system type" and "date". 
Non metric multi-dimensional scaling (nMDS) was performed to represent the samples in a low dimensional space in a way that relative distances of all points are in the same rank order as the relative dissimilarities of the samples as measured by Bray Curtis index. "Stress" values in nMDS indicate how well the multidimensional relationships among the samples are represented in the low dimensional space.

Hierarchical agglomerative clustering with group average linking (based on Bray Curtis similarity) was used to identify "natural groupings" (meant as non-predefined groups) of samples, in such a way that partitioning of groups indicates differences in the microbial community between them. To verify cluster patterns even for the most clearly congregated samples, cluster analysis was used in combination with nMDS plots, as well as the results from the estimation of the components of variation in PERMANOVA.

All statistical analyses were performed by using the multivariate statistical software package Primer V6 (Primer-E Ltd, Plymouth, UK).

\subsection{Results}

During the experiment, the water quality was maintained within preset limits resulting in above $99 \%$ survival. Fish grew on average $11.17 \pm 0.06 \% \mathrm{~g}$ in RAS and $11.03 \pm 0.05 \%$ body weight $\mathrm{d}^{-1}$ in AS with a feed conversion ratio of $0.64 \pm 0.01$ in RAS and $0.70 \pm 0.01$ $( \pm \mathrm{SD}$ ) in AS. The final weight reached was $1.24 \pm 0.03 \mathrm{~g}$ in RAS and $1.17 \pm 0.03 \mathrm{~g}$ in AS. No significant differences were observed between replicate systems, neither for water quality nor for fish growth $(P>0.05)$.

\subsubsection{Overall contribution of factors in microbial dynamics}

PERMANOVA of DGGE data revealed significant effects of all main factors ("system type", "replicate system", "date" and "origin") except for "tank" (Table 2.1). A similar picture emerged for the pyrosequencing data. The highest fraction of total variation was explained by the main factor "origin", followed by "system type", "date" and "replicate system" (Figure 2.1).

Table 2.1. Overall PERMANOVA test based on DGGE data for main experimental factors. P values are based on 9999 Monte Carlo (MC) permutations. Effects of the interaction terms are not shown in the table.

\begin{tabular}{cccc}
\hline Factor & df & Pseudo-F & P(MC) \\
\hline System type & 1 & 5.9632 & 0.0001 \\
Date & 4 & 4.9623 & 0.0001 \\
Origin & 1 & 9.1458 & 0.0001 \\
Replicate system & 5 & 5.6948 & 0.0001 \\
Tank & 8 & 1.0661 & 0.2891 \\
\hline
\end{tabular}


Lowest similarity was observed between gut and water microbiota. Gut samples were separated into RAS and AS systems, confirming that system is the principal factor differentiating gut microbiota. In both systems, gut samples differed significantly between day 07 and 42. Differences between gut samples from "replicate systems" were less pronounced than for "origin", "system type" and "date".

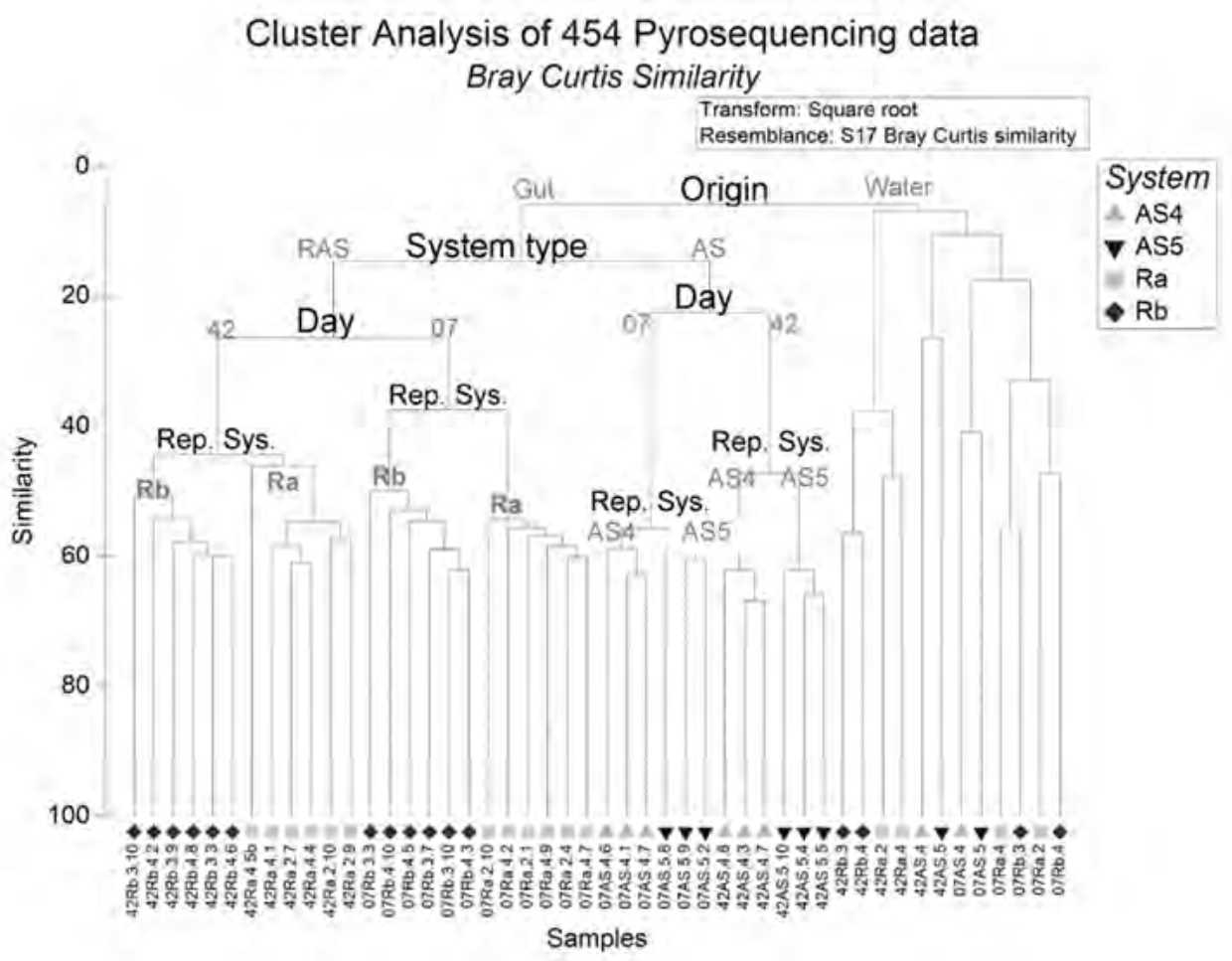

Figure 2.1. Hierarchical clustering with Unweighted Pair Group with Arithmetic Mean (UPGMA) linkage of gut and water samples based on 454 data. On y-axis: similarity percentage based on Bray Curtis similarity, on x-axis: all individual samples of gut (left) and water (right). D07 and 42: sampling day 7 \& 42, AS 4 \& 5: replicate active suspension systems 4 \& 5, Ra and Rb: replicate recirculating system a and b. Numbers 2, 4 \& 3, 4: replicate tanks from $\mathrm{Ra}$ and $\mathrm{Rb}$ respectively. Last digits following the tank number indicate the number of replicate fish in each tank. Since only one water sample was taken from each tank, the last digits were omitted from water sample's ID. (e.g. 42Rb.3.10: Gut of day 42, from recirculating system b, tank 3, fish 10 whereas 07Ra.2: Water of day 7, from recirculating system $\mathrm{a}$, tank 2 )

\subsubsection{Variation in gut and water microbiota from replicate systems}

Recirculating systems (RAS): Gut microbiota of Ra and Rb differed consistently $(\mathrm{P}<0.05$; Table 2.2) during the 42 days experimental period, irrespective of the choice of analytical method (DGGE and 454) or statistical test (PERMANOVA or ANOSIM). The nMDS ordination plots of the DGGE data showed a clear distinction between the microbiota of individuals 
reared in $\mathrm{Ra}$ and $\mathrm{Rb}$ for all dates (Figure. 2.2), confirming the $\mathrm{R}$ statistic in ANOSIM (Table 2.2). The comparison of water microbiota between $\mathrm{Ra}$ and $\mathrm{Rb}$ also differed consistently (Figure 2.3).

\section{Gut Microbiota}
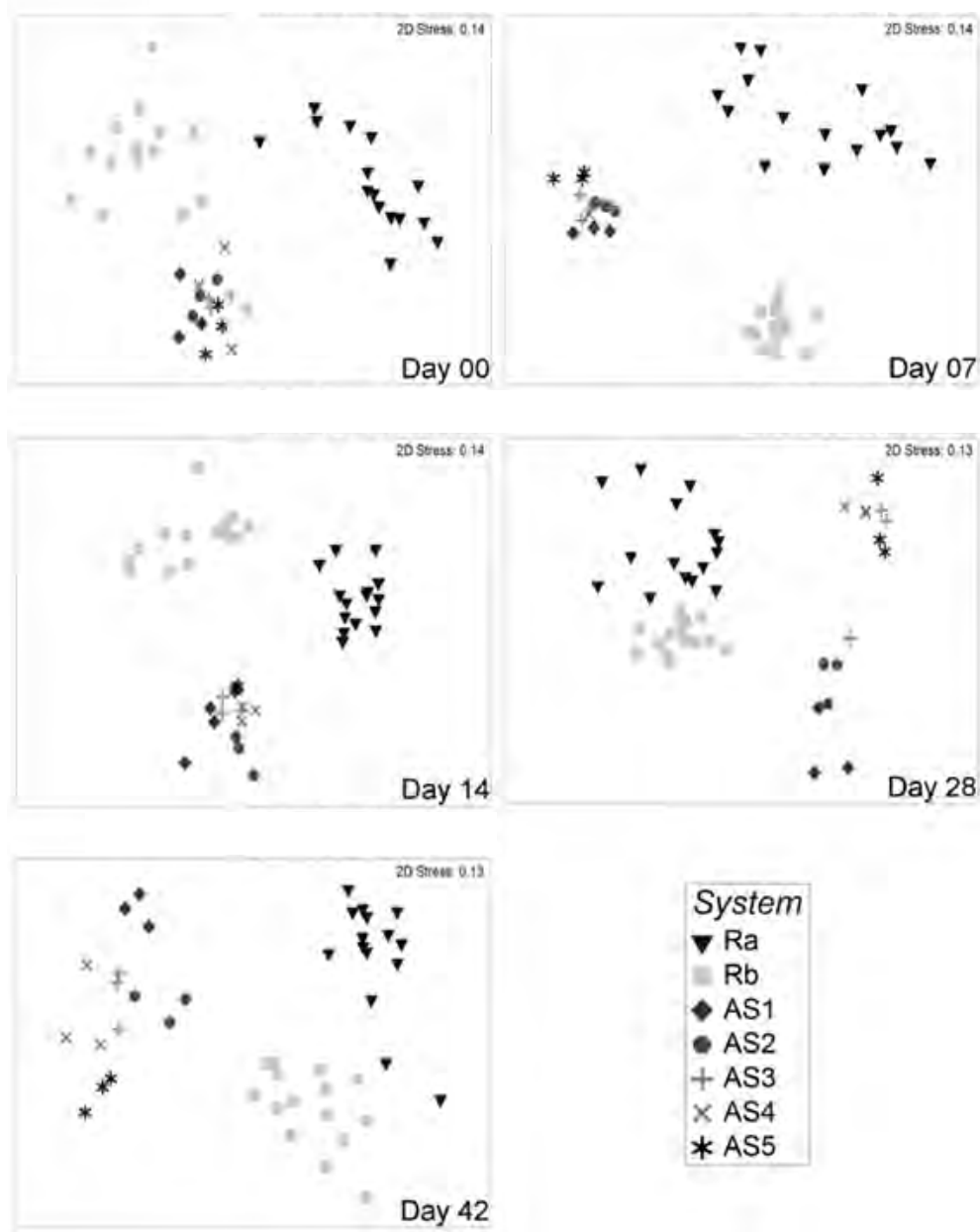

Figure 2.2. Non metric dimensional scaling (nMDS) of gut microbiota from individuals reared in different systems over time. Each point represents the gut microbiota of one individual. Plots are based on Bray Curtis distance after square root transformation of relative abundance DGGE data. D00, 07, 14, 28, 42: sampling days 0 , 7, 14, 28 \& 42 respectively, AS1-5: replicate active suspension system 1 to 5, Ra \& Rb: replicate recirculating system a \& b. Stress values are reported for the two dimension and are indicative of the goodness of fit of data into the plot. 


\section{Water Microbiota}

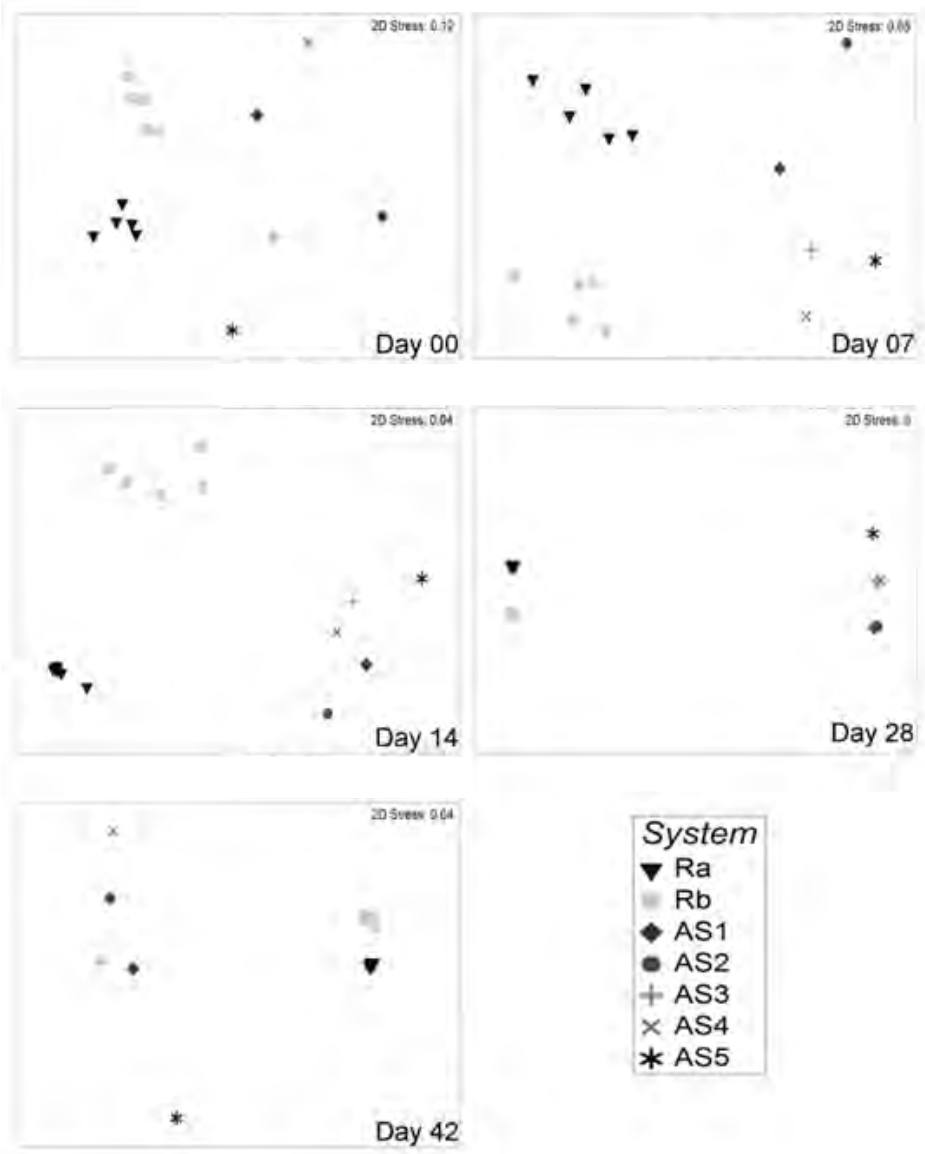

Figure 2.3. Non metric dimensional scaling (nMDS) of water microbiota from different systems over time. Each point represents the water microbiota from each tank. Plots are based on Bray Curtis distance after square root transformation of relative abundance DGGE data. D00, 07, 14, 28, 42: sampling days $0,7,14,28$ \& 42 respectively, AS1-5: replicate active suspension system 1 to 5 , Ra \& Rb: replicate recirculating system a \& b. Stress values are reported for the two dimensions and are indicative of the goodness of fit of data into the plot.

Active suspension systems (AS): Gut microbiota was different between individuals reared in different AS systems at day 07 and day 14, whereas this was not the case on day 0 (Table 2.2). On day 07, the five AS systems were not statistically different when using PERMANOVA. Nevertheless, out of 10 possible pairwise comparisons, six comparisons indicated significant differences between the five AS systems ( $P$ values of each of the pairwise tests are not shown). ANOSIM's R statistic suggested a clear distinction between AS systems for both DGGE and 454 data on day 07. At day 14, the same pattern emerged (6/10 pairwise tests showed differences, and ANOSIM's R statistic was 0.542). 
Table 2.2. Pairwise comparisons between replicate systems for RAS and AS separately, based on DGGE and 454 pyrosequencing data sets. Tests were performed by sample type per sampling day, with $\mathrm{P}$ values for each comparison from two different statistical tests (PERMANOVA and ANOSIM). ANOSIM results are complementary to the PERMANOVA to provide information on the degree of separation between groups, suggested by $R$ statistic. The 2/10, 6/10 \& 7/10 indicate the number of significant out of the total available comparisons. D00, 07, 14, 28, 42: sampling day $0,7,14,28 \& 42$ respectively, AS1-5: replicate active suspension system 1 till 5, Ra \& Rb: replicate recirculating system a \& b. P values are based on 9999 Monte Carlo permutations.

\begin{tabular}{|c|c|c|c|c|c|c|c|c|c|c|c|c|}
\hline \multirow{2}{*}{$\begin{array}{l}\text { Statistical } \\
\text { test }\end{array}$} & \multirow{2}{*}{\multicolumn{2}{|c|}{$\begin{array}{l}\text { Analytical } \\
\text { Method }\end{array}$}} & \multicolumn{2}{|c|}{ D00 } & \multicolumn{2}{|c|}{ D07 } & \multicolumn{2}{|c|}{ D14 } & \multicolumn{2}{|c|}{ D28 } & \multicolumn{2}{|c|}{ D42 } \\
\hline & & & AS1-5 & $\mathbf{R a}-\mathbf{R b}$ & AS1-5 & Ra-Rb & AS1-5 & $\mathbf{R a}-\mathbf{R b}$ & AS1-5 & $\mathbf{R a}-\mathbf{R b}$ & AS1-5 & $\mathbf{R a}-\mathbf{R b}$ \\
\hline \multirow{3}{*}{$\begin{array}{l}\text { Perm. } \\
\text { ANOVA }\end{array}$} & \multirow{6}{*}{ 与 } & DGGE & $2 / 10$ & P:0.0001 & $6 / 10$ & P:0.0003 & $6 / 10$ & P:0.0001 & $7 / 10$ & $\mathrm{P}: 0.0003$ & $7 / 10$ & $P: 0.0001$ \\
\hline & & 454 & & & P:0.2079 & P:0.0025 & & & & & $\mathrm{P}: 0.0242$ & $P: 0.016$ \\
\hline & & ncer & R:0.428 & $\mathrm{R}: 0.925$ & $\mathrm{R}: 0.787$ & $\mathrm{R}: 0.772$ & R:0.542 & $R: 0.922$ & R:0.745 & R:0.596 & R:0.907 & $R: 0.862$ \\
\hline \multirow{3}{*}{ ANOSIM } & & DGGE & P:0.0005 & P:0.0001 & P:0.0001 & P:0.0001 & P:0.0001 & P:0.0001 & P:0.0001 & P:0.0001 & P:0.0001 & P:0.0001 \\
\hline & & & & & $\mathrm{R}: 1$ & $R: 1$ & & & & & $\mathrm{R}: 1$ & $\mathrm{R}: 0.846$ \\
\hline & & & & & P:0.1 & P:0.002 & & & & & $\mathrm{P}: 0.1$ & $\mathrm{P}: 0.002$ \\
\hline \multirow{3}{*}{$\begin{array}{c}\text { Perm. } \\
\text { ANOVA } \\
\text { ANOSIM }\end{array}$} & \multirow{3}{*}{ 岳 } & \multirow{3}{*}{ DGGE } & & $\mathrm{P}: 0.0002$ & & $\mathrm{P}: 0.0212$ & & P:0.0002 & & $\mathrm{P}: 0.0001$ & & $\mathrm{P}: 0.0008$ \\
\hline & & & & $R: 0.996$ & & $R: 0.972$ & & $\mathrm{R}: 1$ & & $\mathrm{R}: 1$ & & $R: 0.988$ \\
\hline & & & & $\mathrm{P}: 0.008$ & & P:0.008 & & $P: 0.008$ & & P:0.008 & & P:0.008 \\
\hline
\end{tabular}

At days 28 and 42, AS systems were different (Table 2.2). Due to lack of replicate samples pairwise comparisons between water samples of AS systems were not possible. To evaluate differences between RAS and AS systems, pooled data of Ra and Rb were compared with pooled data from AS. Pairwise comparisons (Table 2.3) for PERMANOVA and ANOSIM showed that microbiota in gut or water were different between RAS and AS from day 07 onwards $(P<0.001)$.

Table 2.3. Pairwise comparisons between RAS and AS systems based on DGGE and 454 pyrosequencing data sets. Tests were performed by sample type per sampling day, with $P$ values for each comparison from two different statistical tests (PERMANOVA and ANOSIM). ANOSIM analysis is complementary to PERMANOVA as it provides information on the degree of separation between groups, suggested by R statistic. N.A.: No pyrosequencing data available for that day. D00,07,14,28,42: sampling day 0,7,14,28 \& 42 respectively, AS1-5: replicate active suspension system 1 till 5, Ra \& Rb: replicate recirculating system a \& b. P values are based on 9999 Monte Carlo permutations.

\begin{tabular}{|c|c|c|c|c|c|c|c|}
\hline \multirow{2}{*}{$\begin{array}{c}\text { Statistical } \\
\text { test }\end{array}$} & \multirow{2}{*}{\multicolumn{2}{|c|}{$\begin{array}{l}\text { Analytical } \\
\text { Method }\end{array}$}} & \multicolumn{5}{|c|}{ RAS vs. AS } \\
\hline & & & D00 & D07 & D14 & D28 & D42 \\
\hline \multirow{3}{*}{$\begin{array}{l}\text { Perm. } \\
\text { ANOVA }\end{array}$} & \multirow{6}{*}{5} & DGGE & $\mathrm{P}: 0.0710$ & $P: 0.0022$ & P:0.0202 & $\mathrm{P}: 0.0063$ & $\mathrm{P}: 0.0099$ \\
\hline & & 454 & NA & P:0.0152 & NA & NA & P:0.0056 \\
\hline & & DGGF & R:0.244 & $\mathrm{R}: 0.731$ & R:0.496 & R:0.881 & R:0.872 \\
\hline \multirow{3}{*}{ ANOSIM } & & DGUE & P:0.0020 & P:0.0001 & P:0.0001 & P:0.0001 & $\mathrm{P}: 0.0001$ \\
\hline & & & & $\mathrm{R}: 1$ & & & $\mathrm{R}: 1$ \\
\hline & & 454 & NA & P:0.0001 & NA & NA & $\mathrm{P}: 0.0001$ \\
\hline \multirow{3}{*}{$\begin{array}{c}\text { Perm. } \\
\text { ANOVA } \\
\text { ANOSIM }\end{array}$} & \multirow{3}{*}{$\frac{\substack{w \\
\frac{⿱ ㇒}{5}}}{3}$} & \multirow{3}{*}{ DGGE } & P:0.0983 & P:0.008 & P:0.0187 & P:0.0136 & P:0.0062 \\
\hline & & & $\mathrm{R}: 0.820$ & $\mathrm{R}: 0.944$ & $\mathrm{R}: 0.940$ & $\mathrm{R}: 1$ & R:0.990 \\
\hline & & & P:0.0010 & P:0.0010 & P:0.0003 & P:0.0020 & P:0.0020 \\
\hline
\end{tabular}




\subsubsection{Variation in gut microbiota of larvae reared in different tanks}

The extent of variation in gut microbiota of animals reared in replicate tanks of the same Recirculating system was evaluated based on pairwise comparisons of profiles obtained by either DGGE (five tanks per system) or 454 pyrosequencing (two tanks per system). On day 0 , gut microbiota was similar ( $P>0.05$ for all pairwise comparisons) between all replicate tanks in either $\mathrm{Ra}$ or $\mathrm{Rb}$. For all subsequent sampling days, the majority (72\% of all possible comparisons) of the pairwise tests indicated that gut microbiota was not different between replicate tanks ( $P>0.05$, Table 2.4$)$.

Table 2.4. Pairwise comparisons between individuals reared in replicate tanks of the same system. Analysis was performed both on DGGE and 454 pyrosequencing data sets. D00,07,14,28,42: sampling day 0,7,14,28 \& 42 respectively, $\mathrm{Ra} \& \mathrm{Rb}$ : replicate recirculating system a \& b. Tank numbers 6-10 \& 11-15, refer to replicate tanks of Ra and $\mathrm{Rb}$, respectively. P values are based on 9999 Monte Carlo permutations. P values $<0.05$ are highlighted in bold.

\begin{tabular}{|c|c|c|c|c|c|c|c|c|c|c|c|c|c|}
\hline 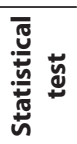 & $\begin{array}{l}\text { Analytical } \\
\text { Method }\end{array}$ & $\begin{array}{c}\text { Ra } \\
\text { tanks }\end{array}$ & D00 & D07 & D14 & D28 & D42 & $\begin{array}{c}\mathbf{R b} \\
\text { tanks }\end{array}$ & D00 & D07 & D14 & D28 & D42 \\
\hline \multirow{11}{*}{ 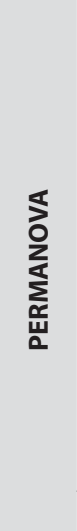 } & \multirow{10}{*}{ DGGE } & $6-7$ & 0.286 & 0.429 & 0.124 & 0.130 & 0.029 & 11-12 & 0.709 & 0.099 & 0.273 & 0.047 & 0.520 \\
\hline & & $6-8$ & 0.535 & 0.494 & 0.155 & 0.026 & 0.009 & $11-13$ & 0.631 & 0.168 & 0.383 & 0.533 & 0.737 \\
\hline & & $6-9$ & 0.217 & 0.042 & 0.026 & 0.081 & 0.011 & $11-14$ & 0.330 & 0.052 & 0.068 & 0.044 & 0.493 \\
\hline & & $6-10$ & 0.365 & 0.017 & 0.043 & 0.298 & 0.009 & $11-15$ & 0.269 & 0.014 & 0.091 & 0.122 & 0.024 \\
\hline & & $7-8$ & 0.338 & 0.260 & 0.295 & 0.177 & 0.298 & $12-13$ & 0.272 & 0.266 & 0.325 & 0.547 & 0.768 \\
\hline & & 7-9 & 0.150 & 0.015 & 0.027 & 0.302 & 0.294 & $12-14$ & 0.212 & 0.071 & 0.013 & 0.049 & 0.341 \\
\hline & & $7-10$ & 0.126 & 0.008 & 0.083 & 0.233 & 0.105 & $12-15$ & 0.114 & 0.017 & 0.028 & 0.228 & 0.024 \\
\hline & & $8-9$ & 0.453 & 0.055 & 0.037 & 0.081 & 0.243 & $13-14$ & 0.604 & 0.149 & 0.151 & 0.557 & 0.596 \\
\hline & & $8-10$ & 0.458 & 0.025 & 0.092 & 0.068 & 0.147 & $13-15$ & 0.383 & 0.055 & 0.187 & 0.653 & 0.074 \\
\hline & & $9-10$ & 0.542 & 0.067 & 0.665 & 0.432 & 0.230 & 14-15 & 0.319 & 0.144 & 0.408 & 0.104 & 0.079 \\
\hline & 454 & $7-9$ & NA & 0.432 & NA & NA & 0.411 & $13-14$ & NA & 0.452 & NA & NA & 0.432 \\
\hline
\end{tabular}

\subsubsection{Temporal dynamics in gut and water microbiota}

The temporal dynamics in gut microbiota was tested separately for $\mathrm{Ra}$ and $\mathrm{Rb}$ and for AS (AS1 to AS5) systems. PERMANOVA on DGGE data revealed a significant impact of time on gut microbiota (Table 2.5). Pairwise comparisons of DGGE patterns obtained for consecutive sampling dates were performed, and for all systems there was a significant time effect for every pairwise comparison (Table 2.5, $\mathrm{P}<0.05$ ). Also for water samples, the temporal variation of microbiota was tested separately for Ra and Rb. At "system type" level, pairwise comparisons of samples taken at consecutive dates indicated that the water-associated microbiota was different between dates $(P<0.05)$ (Table 2.5). 
Table 2.5. Pairwise comparisons between consecutive days for RAS and AS replicate systems separately. Analysis is based on DGGE data sets. Tests were performed by sample type, with $\mathrm{P}$ values for each comparison from two different statistical tests (PERMANOVA and ANOSIM). ANOSIM results are complementary to the PERMANOVA as they provide information on the degree of separation between groups, suggested by $\mathrm{R}$ statistic. D00, 07, 14, 28, 42: sampling day $0,7,14,28$ \& 42 respectively, AS1-5: replicate active suspension system 1 till 5, Ra \& Rb: replicate recirculating system a \& b. P values are based on 9999 Monte Carlo permutations.

\begin{tabular}{|c|c|c|c|c|c|c|c|c|c|}
\hline \multirow{3}{*}{$\begin{array}{c}\text { Statistical } \\
\text { test }\end{array}$} & & \multirow{3}{*}{$\begin{array}{l}\text { Groups } \\
\text { (dates) }\end{array}$} & \multicolumn{5}{|c|}{ AS } & \multicolumn{2}{|c|}{ RAS } \\
\hline & & & AS1 & AS2 & AS3 & AS4 & AS5 & $\mathbf{R a}$ & $\mathbf{R b}$ \\
\hline & & & $P(M C)$ & $P(M C)$ & $P(M C)$ & $\mathbf{P}(\mathbf{M C})$ & $P(M C)$ & (P) & (P) \\
\hline \multirow{4}{*}{ PERMANOVA } & \multirow{5}{*}{5} & 00,07 & 0.010 & 0.001 & 0.005 & 0.010 & 0.003 & 0.002 & 0.006 \\
\hline & & 07,14 & 0.009 & 0.013 & 0.002 & 0.001 & 0.001 & 0.001 & 0.010 \\
\hline & & 14,28 & 0.008 & 0.006 & 0.015 & 0.001 & 0.003 & 0.002 & 0.002 \\
\hline & & 28,42 & 0.010 & 0.005 & 0.033 & 0.008 & 0.005 & 0.005 & 0.004 \\
\hline ANOSIM & & $\begin{array}{c}\text { Global R } \\
\text { (all groups) }\end{array}$ & $\mathrm{R}: 1$ & R:0.997 & R:0.833 & $\mathrm{R}: 1$ & $\mathrm{R}: 1$ & $\begin{array}{l}\text { R:0.906 } \\
\text { P:0.001 }\end{array}$ & $\begin{array}{l}R: 0.938 \\
P: 0.001\end{array}$ \\
\hline \multirow{5}{*}{ PERMANOVA } & \multirow{6}{*}{$\frac{\text { 䍃 }}{3}$} & $\begin{array}{l}\text { Groups } \\
\text { (dates) }\end{array}$ & & & AS & & & $\mathbf{R a}$ & $\mathbf{R b}$ \\
\hline & & 00,07 & & & 0.0079 & & & 0.0003 & 0.0017 \\
\hline & & 07,14 & & & 0.0041 & & & 0.0006 & 0.0009 \\
\hline & & 14,28 & & & 0.0028 & & & 0.0001 & 0.0001 \\
\hline & & 28,42 & & & 0.0091 & & & 0.0003 & 0.0004 \\
\hline ANOSIM & & $\begin{array}{c}\text { Global R } \\
\text { (all groups) }\end{array}$ & \multicolumn{5}{|c|}{ R:0.788 / P: 0.0001} & $\begin{array}{l}R: 0.999 \\
P: 0.0001\end{array}$ & $\begin{array}{c}\text { R:0.989 } \\
\text { P:0.0001 }\end{array}$ \\
\hline
\end{tabular}

\subsubsection{Within group dispersion as a measure of dissimilarity between individuals}

One way ANOVA on Bray Curtis similarity indicated a clear tank or system effect, with individuals being more similar within than between tanks. Between replicate systems the similarity was even lower (Figure $2.4 \mathrm{~A} \& \mathrm{~B}$ ). When comparing similarity between individuals of replicate systems, AS replicate systems were more similar than RAS systems (Figure 2.4 C). These differences in Bray Curtis similarity concurred with differences in group dispersion (Figure 2.4 D). Mean group dispersion indicated that microbiota of individuals reared in AS systems were less dispersed, thus more similar, than for individuals reared in RAS until culture day 28 (Figure $2.4 C \& D$ ). By day 42 the differences in dispersion became non-significant ( $P$ $($ perm $)=0.095)$. There was a significant overall time effect on the group dispersion within system. In AS systems dispersion increased (individuals became less similar) over time (AS: $\mathrm{F}=26.205\left(\mathrm{df}_{\text {system }}: 4, \mathrm{df}_{\text {time*samples }}: 70\right), \mathrm{P}($ perm $\left.)=0.001\right)$, whereas in RAS systems dispersion decreased (RAS: $F=11.683\left(\mathrm{df}_{\text {system }}: 4 \mathrm{df}_{\text {time*samples }^{*}}: 145\right), \mathrm{P}($ perm $\left.)=0.0001\right)$, until the dispersion within the two systems converged on day 28 and 42 (Figure $2.4 \mathrm{D}$ ). These trends were confirmed by the Bray Curtis similarity means over time for AS and RAS (Figure 2.4 C). 


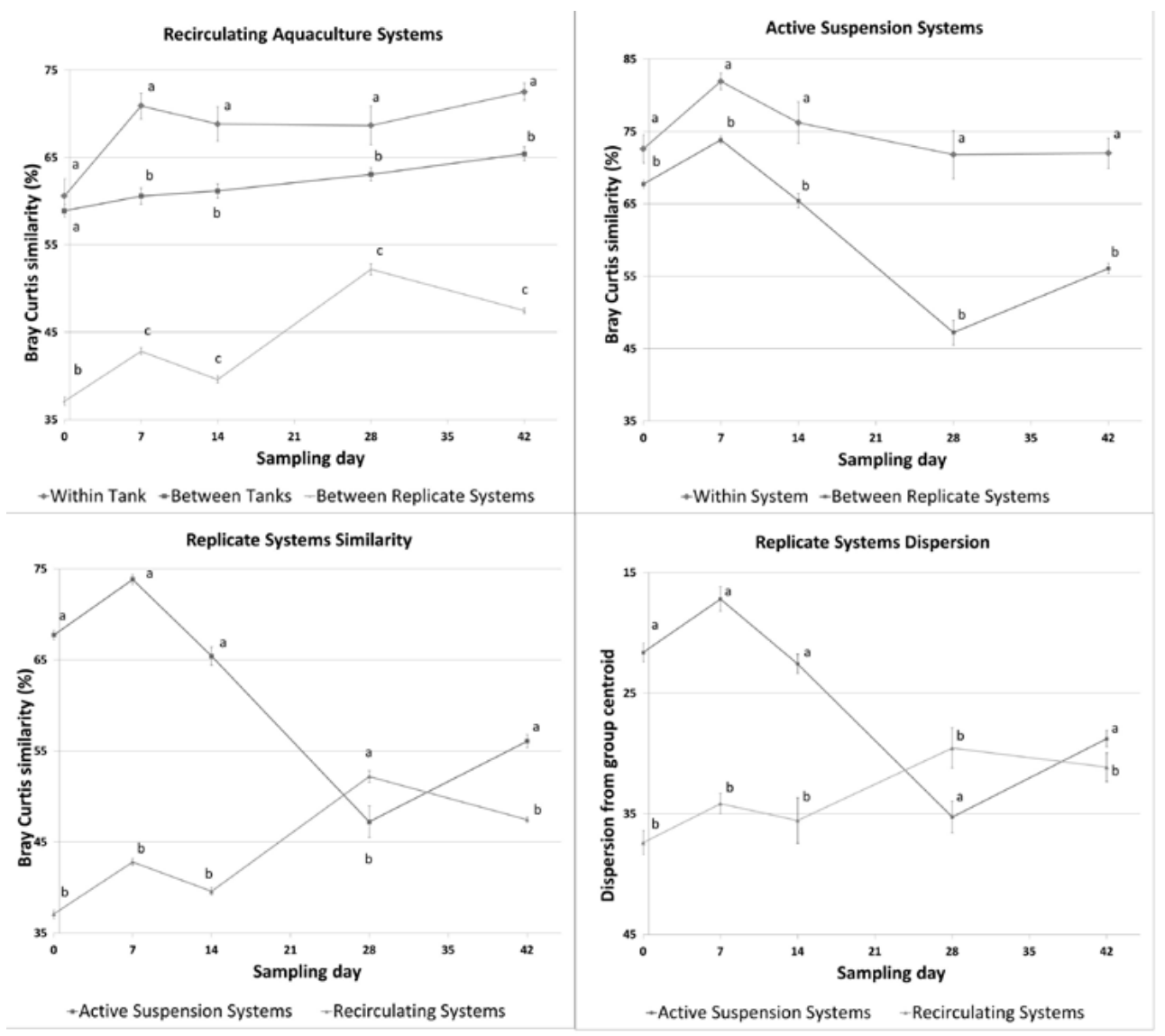

Figure 2.4. Bray Curtis similarity (\%) (A, B \& C) and dispersion from centroid based on Euclidian distance (D) for each sampling day. Points represent mean values of gut microbiota between individuals reared either in the same tank (Within tank), replicate tanks (Between tank) or between systems (Between System). (A): Comparison for Recirculating Aquaculture Systems (RAS). (B) Comparison for Active Suspension (AS). (C) Comparison between RAS and AS Systems \& (D) Dispersion of samples from group centroid in RAS and AS systems. Error bars show standard error. Different data labels ( $a$, $b$ and $c)$ per sampling day indicated significant difference $(P<0.05)$ based on one-way ANOVA and Bonferroni ranking test for $A, B \& C$ and permutation dispersion test for $D,(P($ perm $)<0.05)$.

\subsection{Discussion}

In this study we characterized the spatio-temporal variation in the gut microbiota of tilapia larvae, reared in two different types of aquaculture systems. As was mentioned in the introduction, a subset of samples was analyzed using two different analytical methods, namely DGGE and pyrosequencing of PCR-amplified 16S rRNA gene fragments. The comparison revealed that data obtained by both methods were not contradicting each other. DGGE as a method has some specific limitations, for instance, the separation of relatively small DNA fragments, the co-migration of DNA fragments with different 
sequences, the detection of hetero-duplex molecules and the limited sensitivity of detection of rare community members (Muyzer, 1999). In addition, multiple comparisons among different DGGE gels might lead to a false positive conclusion due to high gel to gel variation (Muyzer et al., 1993; Ferris et al., 1996). However, in this study these errors were small and did not jeopardize the broader picture, as it was also confirmed by the pyrosequencing data analysis.

\subsubsection{Differences in gut and water microbiota in different tanks}

The results showed that variation in individual gut microbiota within tanks was similar to the variation between tanks. This can probably be explained by the fact that larvae shared the water source and feed. To this end, it is interesting to note that pairwise comparisons indicated that the microbiota in larvae reared in replicate tanks in the same RAS were mostly similar, but not in all cases (Table 2.4). Bakke et al. (2013), pointed out that when sampling a few fish in only two replicate tanks, on one occasion gut microbiota differed between two tanks, whereas on another occasion gut microbiota was similar between two other tanks. When sampling only a few individuals in only two tanks the power of the analysis is low. We sampled only three individuals per tank resulting in 10 possible unique permutations. This allows only for a maximum significance level of $10 \%$ and in such cases Monte Carlo permutation was used. Nevertheless, unique permutation based $P$ values are preferred when the minimum significance level drops below $1 \%$; this will be realized when sampling a minimum of individuals per tank. Anderson et al. (2006) suggested that examining average within/between group dissimilarities and dispersion, as well as using unconstrained ordination plots, helps to reveal the nature of differences among groups detected by PERMANOVA. In our case, nMDS plots of the DGGE data and the cluster analysis of the pyrosequencing data did not show a clear separation of gut microbiota between larvae reared in replicate tanks. Moreover, ANOSIM's R statistic of tank pairwise comparisons was very low or even negative, also suggesting there is no tank effect on gut microbial communities. Bakke et al. (2013), in contrast to our findings, reported differences in gut microbiota between replicate tanks. This might be due to cumulative differences in water microbiota between replicate tanks and variation in microbiota of daily fed live feeds as opposed to the pelleted commercial diet used in our study. Another reason might be that Bakke et al. (2013), extracted DNA from whole cod larvae after homogenization. Although larvae were disinfected externally, the possibility of contamination cannot be excluded. In our study, fish guts from comparatively much larger tilapia larvae were dissected aseptically after sterilizing body surfaces, with lower risk for contamination. High within tank variation in gut bacteria of cod larvae was also reported by Fjellheim et al. (2011). Here too, larvae were fed live feeds, and whole larvae were used for DNA extraction. In addition, larvae were sampled only from one tank per treatment, and conclusions were drawn based on a combination of culture dependent 
and independent techniques. These results should be considered with caution, because the cultivability of microbiota varies with species composition.

\subsubsection{Differences in gut and water microbiota between replicate systems}

Gut microbiota between replicate AS systems became different within one week $(P<$ $0.05)$, whereas gut microbiota of the individuals reared in $\mathrm{Ra}$ and $\mathrm{Rb}$ was different already from day 0 ( $P<0.05 ; 43.8 \% \pm 0.26$ SE Bray Curtis similarity). Microbiota in water was also different $(P<0.05)$ between $\mathrm{Ra}$ and $\mathrm{Rb}$. Verschuere et al. (1997), monitored the water microbial communities in three identical Artemia culture series, showing distinct microbial communities developing in each of them, suggesting differentiation is stochastic. This concurs with the observed differences of microbiota in gut and water between replicated RAS or AS systems in this study (Figure 2.1). On each sampling day, based on their gut microbiota, larvae reared in Ra differed from those reared in Rb. Similarly, larvae reared in AS4 and AS5 differed (Figure 2.1; P $<0.05$ ). This difficulty to replicate systems when studying individual gut microbiota makes experimental design challenging.

In our study, water quality parameters and fish growth were not significantly different between replicate systems (data not shown), yet their microbial communities differed. The observed differences in microbial composition do not necessarily imply differences in functionality (Mouchet et al., 2012). Functional redundancy suggests that functional diversity of an ecosystem is additive when species are complementary, or decreases, when species share functions (Bell et al., 2005). Our results suggest that different treatments (for example, testing dietary effects on gut microbiota) should preferably be tested in tanks within the same system, to reduce variation due to system replication.

\subsubsection{Differences in gut and water microbiota between different types of rearing systems}

Except for day 0, water and gut microbiota differed between RAS and AS, suggesting a clear system effect. Larval growth, feed conversion and survival between RAS and AS were similar (data not shown), and it is thus safe to assume that observed differences in gut microbiota were not caused by growth related factors or health status of the larvae. Regarding water, rearing system type also affected microbial communities. Possible underlying mechanisms will be explored in a separate paper focusing on differences in bacterial community species composition based on pyrosequencing data.

One question is whether differences in gut microbiota can be explained by differences in water microbiota. Water microbiota, together with feed microbiota, have a large impact on gut microbiota in early life stages (Hansen and Olafsen, 1999). Bakke et al. (2013), suggested that relatively small differences in water microbiota may impose significant 
differences in larval microbiota, and this might also be the case in our study. Cluster analysis of Bray Curtis similarity of relative abundance data showed that only $10 \%$ of the gut and water microbiota was overlapping (Figure 2.1). Nevertheless, species subdominant or even below the detection threshold in the water might be dominant in the gut, or vice versa.

The lack of significant differences in gut microbiota between RAS and AS on day 0 might be due to two reasons; i. high similarity between the microbial communities of the two systems or ii. high within system variation (dispersion). Anderson (2006) suggested that PERMANOVA test should be used combined with a test of homogeneity of multivariate dispersion (PERMDISP). Our results showed that dispersion in RAS was significantly higher at day 0 , (compared to the rest of the days), and this was most likely the reason that gut microbiota from larvae reared in RAS did not differ significantly from the ones reared in AS. This might as well explain why water microbial communities between the two systems did not differ on day 0 , as PERMDISP confirms that water microbiota among the five different AS systems was more dispersed on day 0 than on any other day of the experiment.

\subsubsection{Temporal variation in gut and water microbiota}

It is interesting to observe, that in spite of the enormous changes during early development, the effect of "date" was not the most pronounced among factors. The "date" effect to a large extend is linked to structural and functional changes of the gut during early development, including changes in the gut microbiota (Wilson and Castro, 2010). Changes could be induced by fluctuations in $\mathrm{pH}$, gastric secretions and digestive enzymes activity, presence of bile salts, nutrients availability (from endogenous to exogenous feeding), as well as some stochastic events (Hansen and Olafsen, 1999; Verschuere et al., 1997).

When plotting the temporal trajectories of gut microbiota of larvae reared in RAS and AS, both systems clearly differed from day 07 onwards. While the trajectories of the five AS systems were very different, changing almost stochastically (plots not shown), the two replicate RAS trajectories were similar even if the two replicate RAS did not share the same water source (Figure 2.5). 
Gut Microbiota (Recirculating Systems RAS) Time Trajectory of Groups Centroid
Gut Microbiota (Active Suspension Systems AS)

Time Trajectory of Groups Centroid

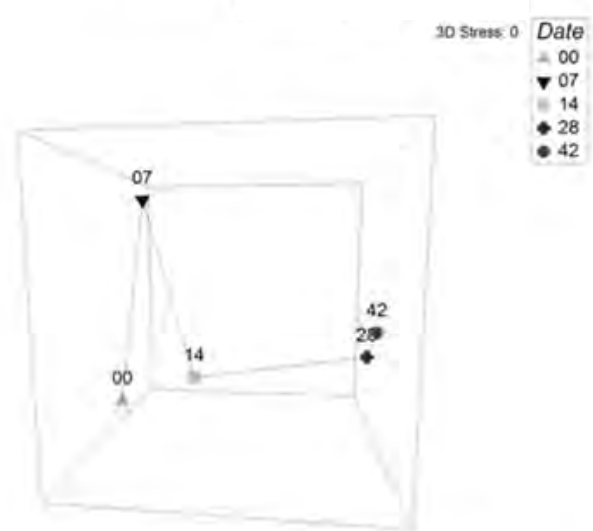

Gut Microbiota (Recirculating System Ra) Time Trajetory of Groups Centroid

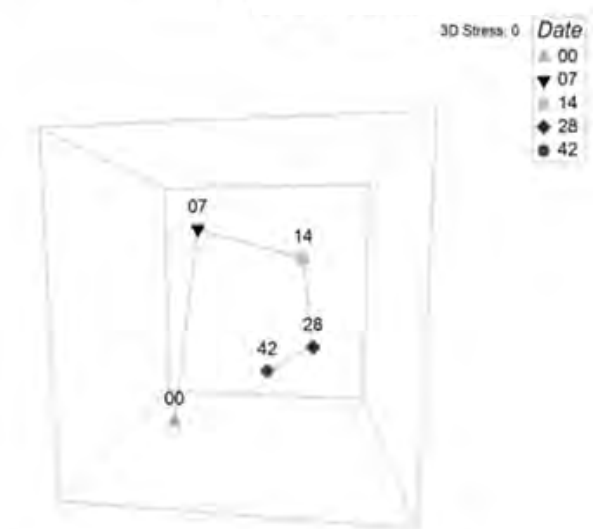

Gut Microbiota (Recirculating System Rb) Time Trajectory of Groups Centroid
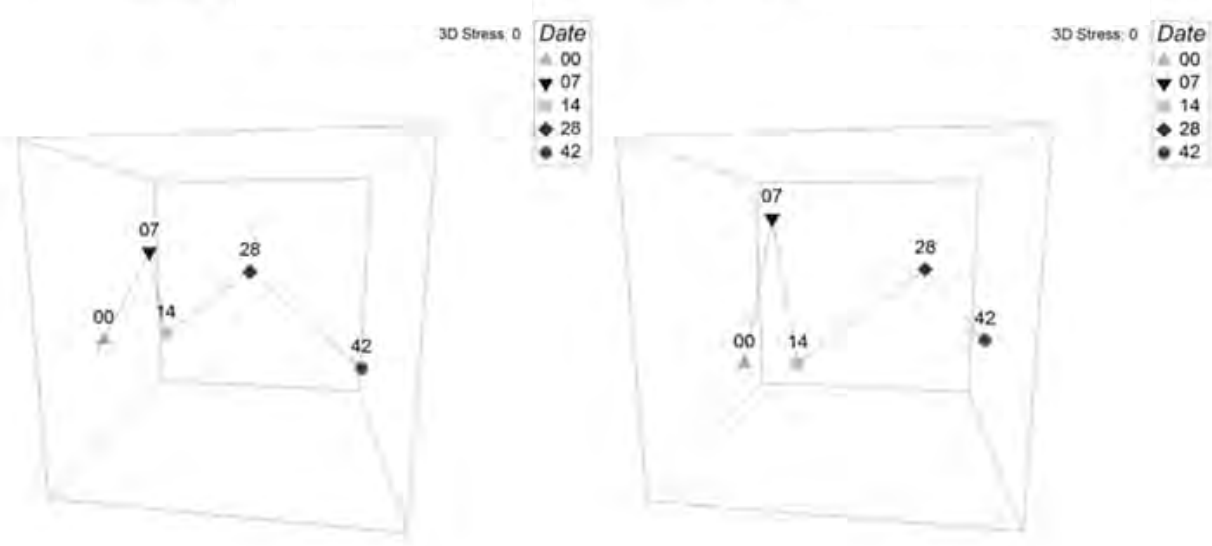

Figure 2.5. Three dimensional nMDS plots of gut microbiota from different systems over time (trajectory). Plots are based on Bray Curtis distance after square root transformation of relative abundance DGGE data. D00, 07, 14, 28,42 : sampling days $0,7,14,28 \& 42$ respectively. Each point in the plots represents the group centroid and the shift of group average microbiota in time. Zero stress values for each plot are indicative of the fit due to the representation of the centroids. AS and RAS: active suspension and recirculating system, Ra \& Rb: replicate recirculating system $a \& b$.

There was a clear distinction of day $0,7 \& 14$ from days 28 and 42 for the RAS systems, whereas for the AS systems such a separation was not evident. This agrees with the observed overall (all five points) lower dispersion of AS gut samples compared to dispersion of RAS gut samples. This might be due to two reasons; i. Gut microbiota changed less over time in AS than in RAS; ii. Microbiota of individuals was more similar on each sampling day in AS than in RAS. A possible explanation is that in AS systems 
solids remain in the fish tank and most of the organic carbon and nitrogen is available for heterotrophic bacteria. These bacteria are abundant in high concentrations in the water reaching densities of $10^{7} \mathrm{CFU} \mathrm{mL}^{-1}$ (Burford et al., 2003). Bacteria, protozoa, algae and zooplankton form bioflocs, are directly available to fish (2007). Grazing on bioflocs might have caused gut microbiota in AS to be more uniform than in RAS.

\subsection{Conclusions}

Microbiota in water and in larval guts between replicate systems was very different. When individuals share the same water, the rearing tank had a minor effect on gut microbiota. Compositional replication of the microbial communities at system level was not successful. Apparently, our understanding and control of underlying deterministic and stochastic factors is insufficient. This poses many challenges when researching treatment effects on gut or water microbiota. We recommend to investigate treatment effects on gut microbiota within the same system (fish share the same water source), rather than between replicate systems, unless systems can be replicated within treatment. Our results showed that gut microbiota of individuals between tanks of the same system did not differ, whereas between replicate systems they did. The observed rapid and stochastic changes of microbiota in gut and water over time, suggests that long term studies should be interpreted carefully. Observations of start and endpoint do not provide information about the temporal variation in between.

\section{Acknowledgements}

We would like to thank Hans Heilig, Menno Ter Veld and Muhamad Amin for their contribution on samples collection and laboratory analysis. We also thank all participants of the EU-project Promicrobe, whose enthusiasm and expertise helped to shape ideas expressed in this manuscript. 


\section{Supplementary material}

\begin{tabular}{|c|}
\hline Factor \\
\hline $\begin{array}{c}\text { System } \\
\text { Type }\end{array}$ \\
\hline $\begin{array}{c}\text { Replicate } \\
\text { System }\end{array}$ \\
\hline Tank \\
\hline Origin \\
\hline Date \\
\hline
\end{tabular}

\section{Schematic overview of experimental
factors and levels}

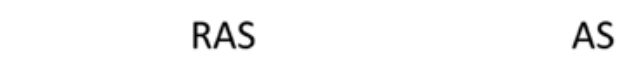

(Recirculating Aquaculture System) (Active Suspension)
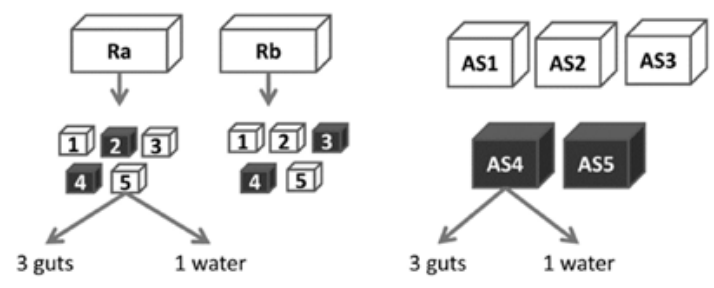

Figure S2.1. Schematic overview of experimental factors and levels. Five active suspension (AS) and 2 recirculating aquaculture systems (RAS) were used. The replicate RAS are named Ra and Rb; the replicate AS systems are named AS1 through AS5. Each RAS contained five tanks which shared the same water source. AS systems did not have sub-divisions. For DGGE analysis, three guts and water were sampled from each tank in RAS (10 tanks total) and each AS (5 systems) on sampling day 00, 07, 14, 28 \& 42. Sub-sets of samples for DGGE of Ra2, $\mathrm{Ra} 4, \mathrm{Rb} 3$ and Rb4 (dark shaded tanks), and active suspension systems AS4 and AS5 (also dark shaded), taken on days 07 and 42, were used for pyrosequencing.

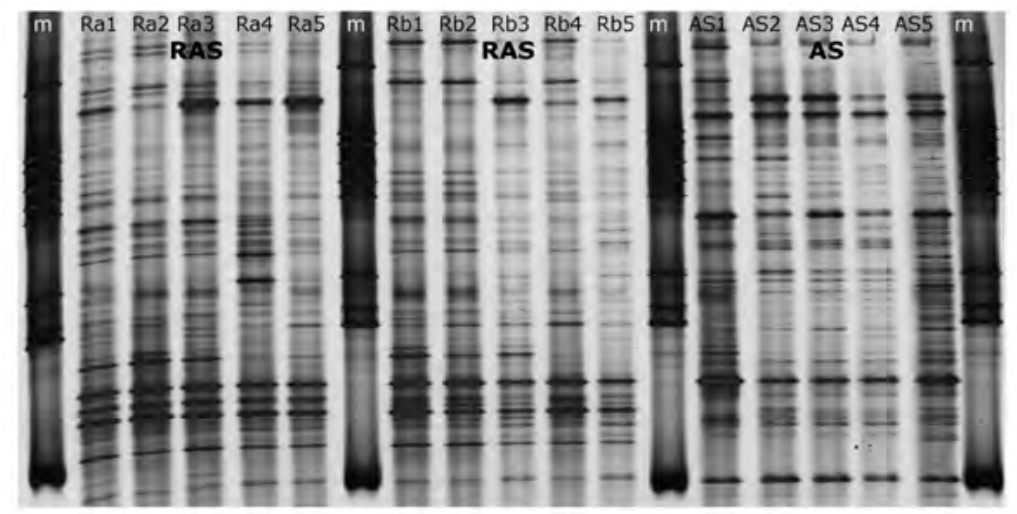

Figure S2.2. Denaturing gradient gel electrophoresis (DGGE) of gut microbiota on day 28. Each lane displays the banding pattern (fingerprint) of gut microbiota from an individual fish. Samples were taken from different systems on experimental day 28. Ra and Rb: Recirculating aquaculture systems $a$ and $b$, from tank 1 to 5, AS1-5: Active suspension systems 1 through 5, m: standard reference marker consisting of an amplicon mix of 10 different cloned bacterial $16 \mathrm{~S}$ rRNA genes used for digital gel normalization. 


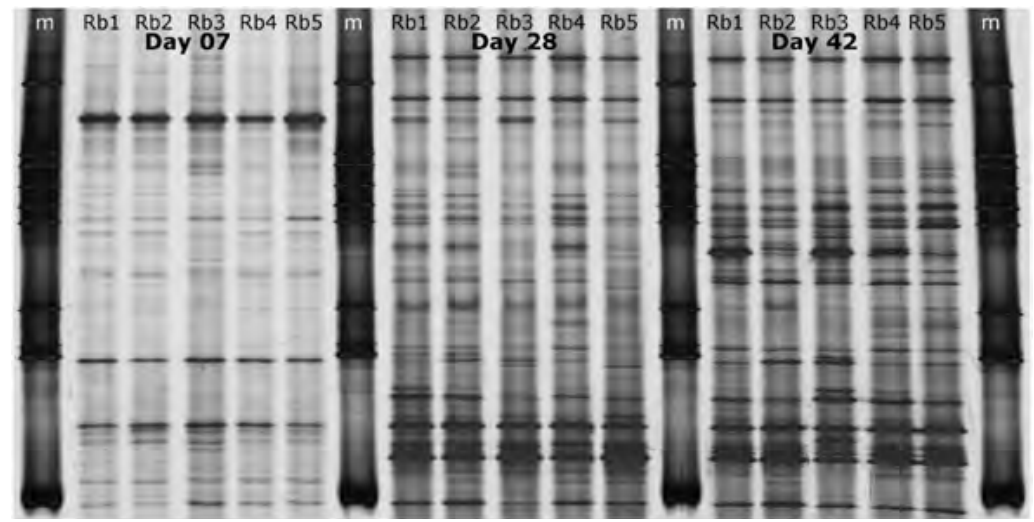

Figure S2.3. Denaturing gradient gel electrophoresis (DGGE) of gut microbiota of system Rb over time. Each lane displays the banding pattern (fingerprint) of gut microbiota from an individual fish. Samples were taken from the same system on experimental day 07,28 and 42 . Rb: Recirculating aquaculture systems $b$, from tank 1 to 5 , m: standard reference marker consisting of an amplicon mix of 10 different cloned bacterial $16 \mathrm{~S}$ rRNA genes used for digital gel normalization.

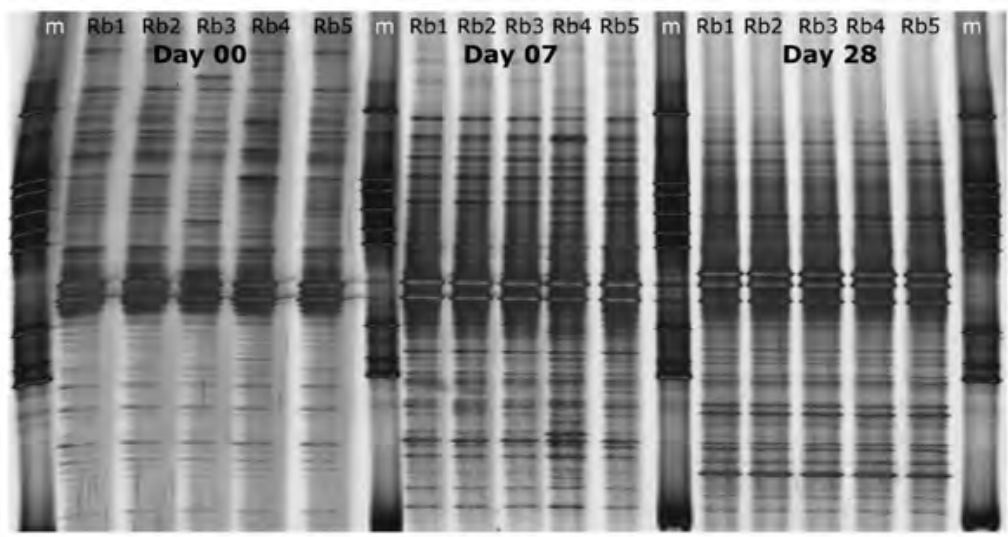

Figure S2.4. Denaturing gradient gel electrophoresis (DGGE) of water microbiota from system Rb over time. Each lane displays the banding pattern (fingerprint) of water microbiota from each tank. Samples were taken from the same system on experimental day 00,07 and 28 . Rb: Recirculating aquaculture systems $b$, from tank 1 through 5, m: standard reference marker consisting of an amplicon mix of 10 different cloned bacterial $16 \mathrm{~S}$ rRNA genes used for digital gel normalization. 


\section{Chapter 3}

The impact of rearing environment on the development of gut microbiota in tilapia larvae

i
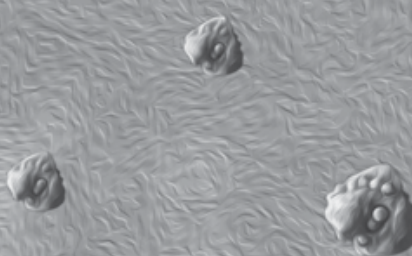

This chapter has been published as:

Christos Giatsis, Detmer Sipkema, Hauke Smidt, Hans Heilig, Giulia Benvenuti, Johan Verreth, Marc Verdegem (2015) The impact of rearing environment on the development of gut microbiota in tilapia larvae. Sci. Rep. 5, 18206; doi: 10.1038/ 
This study explores the effect of rearing environment on water bacterial communities (BC) and the association with those present in the gut of Nile tilapia larvae (Oreochromis niloticus, Linnaeus) grown in either recirculating or active suspension systems. 454 pyrosequencing of PCR-amplified 16S rRNA gene fragments was applied to characterize the composition of water, feed and gut bacteria communities. Observed changes in water BC over time and differences in water $\mathrm{BC}$ s between systems were highly correlated with corresponding water physico-chemical properties. Differences in gut bacterial communities during larval development were correlated with differences in water communities between systems. The correlation of feed $\mathrm{BC}$ with those in the gut was minor compared to that between gut and water, reflected by the fact that 4 to 43 times more OTUs were shared between water and gut than between gut and feed BC. Shared OTUs between water and gut suggest a successful transfer of microorganisms from water into the gut, and give insight about the niche and ecological adaptability of water microorganisms inside the gut. These findings suggest that steering of gut microbial communities could be possible through water microbial management derived by the design and functionality of the rearing system. 


\subsection{Introduction}

Gut microbiota influences a wide range of biological processes of their host, including digestion, innate immunity, proliferation of epithelial cells and structural and functional maturation of the gut in humans (Rawls, 2007; Sekirov et al., 2010), domesticated terrestrial animals (Chaucheyras-Durand and Durand, 2010; McFall-Ngai et al., 2013) and fish (Nayak, 2010b; Sullam et al., 2012). However, our understanding of the roles and drivers for community diversity and species dominance within the gut is presently limited (Walker et al., 2011).

Fish are exposed to higher microbial loads in the aquatic environment than terrestrial domesticated animals are in air or soil (Verschuere et al., 2000). Thus, this closer contact with the surrounding water likely affects early gut colonization. Whilst water seems to affect the fish gut microbiota already from mouth opening onwards (Reitan et al., 1998), feed microbiota becomes important at a later development stage (Hansen and Olafsen, 1999; Ringo and Birkbeck, 1999). However, although water and feed are the two main sources of microorganisms available to fish, the factors underlying the successful colonization of ingested microbes and the community assembly inside the gut are still poorly understood (De Schryver and Vadstein, 2014). For this reason, predictability and repeatability of gut microbiota manipulations is currently limited.

The nutrient rich environment of high density aquaculture is conducive to the proliferation of microbes. To this end, aquaculture management aims to minimize the amount of nutrients in the rearing tank. For example, in recirculating aquaculture systems (RAS) the first step of water purification is solids removal (Crab et al., 2007; Gutierrez-Wing and Malone, 2006). In contrast, in active suspension (AS) systems, fish excreta and feed left overs are mineralized inside the rearing tank. AS systems are characterized by the formation of bioflocs (aggregates of microorganisms, colloids, organic polymers and dead cells), which contribute to the maintenance of good water quality while providing additional nutrients to the fish (De Schryver et al., 2008).

In the present study, we hypothesized that water bacterial communities would differ between the two rearing environments (i.e. RAS vs. AS systems) due to differences in systems' design. Assuming that gut colonization is strongly affected by the composition of the microbiota in the water, we expected that composition of gut communities would differ between systems as well. Compared to RAS, bacteria in the water of AS systems have a higher chance of being successfully transferred in the gut, due to the constant grazing of larvae on bioflocs. Thus, a greater influence of AS water microbiota on the gut is expected. We used 454 pyrosequencing of PCR-amplified bacterial $16 \mathrm{~S}$ ribosomal RNA (rRNA) gene fragments to characterize for the first time the composition of gut BCs of Nile tilapia Oreochromis niloticus (Linnaeus) reared for 42 days in RAS or AS tanks from 
the moment of first feeding. The progressive gut colonization in fish reared in the two systems and the similarity of water and feed BCs with those in the gut were evaluated at different time points. Furthermore, the potential role of the most predominant bacteria found in this study is discussed in detail based on a comprehensive literature review.

\subsection{Materials and methods}

\subsubsection{Experimental animals and set up}

The experiment was performed in accordance with relevant guidelines and approved by the Ethical Committee of Wageningen University for animal experiments (Registration code: $2009055 \mathrm{~d}$ ). One batch of three days post-fertilization ( $3 \mathrm{dpf}$ ) eggs were washed out from the mouth of a female Nile tilapia and incubated for four days at $27^{\circ} \mathrm{C}$. Eggs were incubated in a tank until swim-up stage and received water from the same source. For rearing the larvae, two different culture systems were used: a recirculating aquaculture system (RAS) and an active suspension (AS) system with two replicates each (Ra\&Rb and AS1\&AS2), which were initially filled with the same water coming from a well. Replicate systems did not share the same water. Only for RAS systems, each replicate also contained 2 replicate tanks ( $R a 1, R a 2 \& R b 1, R b 2$ ) which were connected to the same water treatment unit (biofilter). In each tank, 100 randomly selected swim-up larvae (7 dpf) were introduced before first feeding and before mouth opening. Mouth opening occurred within the experimental rearing systems. Feeding started $9 \mathrm{dpf}$ (referred to as day 0) and was continued for 42 days. Fish were fed three times a day to apparent satiation with a starter feed (F-0.5 GR Pro Aqua Brut - Trouw Nutrition, France; 57\% crude protein, 15\% crude fat, $8.5 \%$ carbohydrates, $11 \%$ crude ash, $0.6 \%$ crude fiber, $1.7 \%$ phosphorus). A 42 day culture period was considered sufficiently long for all major stages of the gut development (Fujimura and Okada, 2007). Water, feed and gut samples were collected on day 7 and 42. Further details about the experimental setup, animals and description of the rearing systems can be found elsewhere (Giatsis et al., 2014).

\subsubsection{Sampling of guts, water and feed}

On sampling days, three tilapia larvae were randomly collected from Ra1, Ra2, Rb1, Rb2, AS1 and AS2 for gut BC analysis. After euthanization, the larvae were rinsed with $70 \%$ ethanol and sterile water, before dissecting the gut. Gut samples were flash-frozen in liquid nitrogen and stored individually at $-80{ }^{\circ} \mathrm{C}$ until further processing. Furthermore, $250 \mathrm{~mL}$ of water was collected from each tank. Water was simultaneously filtered through a 0.45 $\mu \mathrm{m}$ (type HAWP) over a $0.22 \mu \mathrm{m}$ (type GTTP) membrane filter (Millipore - Isopore ${ }^{\mathrm{TM}}$ ) using a vacuum apparatus. Filters were stored at $-80^{\circ} \mathrm{C}$ until processing. The $0.45 \mu \mathrm{m}$ filters were 
used in order to avoid clogging of the $0.22 \mu \mathrm{m}$ with large size particles during filtration. However, both filters were used for DNA extraction from water. Finally, also 2 grams of feed were collected and stored at $-80^{\circ} \mathrm{C}$ until processing. All gut, water and feed samples were stored and analyzed individually. Samples were not pooled neither were the corresponding DNA extracts.

On a weekly basis, total ammonia nitrogen (TAN-N), nitrite $\left(\mathrm{NO}_{2}^{-}-\mathrm{N}\right)$, nitrate $\left(\mathrm{NO}_{3}^{-}-\mathrm{N}\right)$, orthophosphate $\left(\mathrm{PO}_{4}^{3-}\right)$, carbon dioxide $\left(\mathrm{CO}_{2}\right)$, urea, dissolved oxygen (DO), $\mathrm{pH}$, conductivity and temperature $\left({ }^{\circ} \mathrm{C}\right)$ were measured in each tank according to Giatsis et al. (2014).

\subsubsection{Isolation of DNA}

DNA was extracted from larval gut samples using the DNeasy Blood \& Tissue Kit (Qiagen, Venlo, Netherlands), whereas the FastDNA SPIN kit for soil (MP Biomedicals, Ohio, USA) was used for water and feed samples. The use of different physical, mechanical, chemical and enzymatic lysis protocols, different purification and precipitation techniques and different reagents, have shown to affect genomic DNA yield and quality. This was confirmed by Lever et al. (2015), however, the authors furthermore concluded that the observed effects are almost always sample dependent. This being said, before our study, genomic DNA was extracted from all sample types by using two extraction protocols. Since the DNeasy Blood \& Tissue Kit resulted in a very low DNA yield from the water and feed samples and a high DNA yield was obtained with the FastDNA SPIN kit for soil, and hence the latter was used for all water and feed samples in this study. In contrast, the FastDNA SPIN kit for soil showed a low yield for DNA extraction from gut samples, while a high yield was obtained with the DNeasy Blood \& Tissue Kit. Therefore, the DNeasy Blood \& Tissue Kit was used for all gut samples in this study.

Modifications of the manufacturer's protocol with respect to enhancement of cell lysis (i.e. bead beating), DNA purification and elution processes, were made as previously described by Giatsis et al. (2014).

\subsubsection{PCR and 454 Pyrosequencing}

Barcoded amplicons from the V1-V2 region of $16 \mathrm{~S}$ rRNA genes were generated by PCR using the 27F-DegS primer (Van den Bogert et al., 2011) appended with titanium sequencing adaptor $A$ and an $8 \mathrm{nt}$ sample-specific barcode (Hamady et al., 2008) at the $5^{\prime}$ end. A detailed protocol for the PCR is given by Giatsis et al. (2014). Nucleotide sequences were generated by pyrosequencing using an FLX genome sequencer in combination with titanium chemistry (GATC-Biotech, Konstanz, Germany). These sequence data were submitted to the European Bioinformatics Institute under study accession No PRJEB4462 and sample accession No ERS343984 - ERS344037. 


\subsubsection{Data handling}

Pyrosequencing data were analyzed using the QIIME 1.5.0 pipeline (Caporaso et al., 2010) and quality filtering was performed as follows: low quality sequences were removed using default parameters. Removed reads included (i) reads with less than 200 or more than 1000 nucleotides; (ii) reads with more than 6 ambiguous nucleotides, homopolymer runs exceeding 6 bases, reads with missing quality scores and reads with a mean quality score lower than 25; and (iii) reads with mismatches in the primer sequence. Operational taxonomic units (OTUs) were identified at the $97 \%$ identity level. Representative sequences from the OTUs were aligned using PyNAST (DeSantis et al., 2006). The taxonomic affiliation of each OTU was determined using the RDP classifier at a confidence threshold of $80 \%$ against the 12_10 Greengenes core set (Wang et al., 2007). Possible chimeric OTUs were identified using QIIME's ChimeraSlayer and removed from the initially generated OTU list, producing a final set of non-chimeric OTUs.

To visualize possible commonalities and anti-correlations between gut, water and feed BCs, physical samples and OTUs were plotted as nodes in a bipartite network. In the network analysis, the average relative abundance of OTUs in replicate samples was represented. To cluster the OTUs and hosts in this network, the Edge-weighted Spring Embedded algorithm as implemented in Cytoscape 3.0.2 was used (Saito et al., 2012).

\subsubsection{Statistical analysis}

A repeated measure ANOVA was applied for water quality measurements on day 7 and 42 with "system" (RAS or AS) as main factor and "day" (7 or 42) as repeated measure. Based on Draftsman plots (variable pair-wise scatter plots) of water quality data, the TAN values were log-transformed (Figure S3.1). Subsequently, all environmental variables were normalized. The Euclidean distance of normalized environmental variables was used as dissimilarity measure. Gut, water and feed BC data were expressed as relative abundance of all OTUs in each sample, and Bray Curtis similarity was calculated based on square root transformed data.

A distance-based linear model (distLM) was used for analyzing and modelling the relationship between the bacterial community composition, as described by a resemblance matrix, and the predictor variables (i.e. water parameters). When not enough possible permutations were available, P-values for testing the null hypothesis of no relationship between the two datasets were obtained by Monte Carlo permutations (a sample was drawn from the theoretical asymptotic permutation distribution) (Anderson and Robinson, 2003). Forward selection of the environmental variables was applied and distance-based redundancy analysis (dbRDA) was used for visualization (Legendre and Anderson, 1999; Anderson et al., 2006). 
Based on Bray Curtis similarity of relative abundance data, RELATE analysis evaluated the relatedness between water and gut BC by calculating Spearman's $\rho$ correlation coefficient between all elements. Similarity percentages (SIMPER) analysis was used to determine the OTUs which contributed most to the discrimination or relatedness of samples in each group. To measure alpha-diversity of the bacterial communities: the total number of observed species $(S)$, Pielou's evenness $\left(J^{\prime}=H^{\prime} / \log (S)\right)$ and Shannon $\left(H^{\prime}=-S U M\left(P_{i}^{*} \operatorname{In}\left(P_{i}\right)\right)\right)$ were calculated $\left(\mathrm{P}_{\mathrm{i}}\right.$ is the proportion of the total count (abundance) arising from the ith species). As species richness and evenness can only be compared between samples when sample sizes are equal (Hurlbert, 1971), resulting reads were randomly selected so as to standardize to the sample with the least number of obtained reads $(n=1719)$. To visualize community evenness, dominance plots were created based on relative abundance data. OTU relative abundance (y-axis) was averaged per sample type (gut and water), for each system (AS and RAS) per day (7 and 42). Cumulative relative abundance was plotted against the increasing species rank ( $x$-axis).

Statistical analyses were performed using Primer 6 (version 6.1.11) (Primer-E Ltd., Plymouth, United Kingdom) and its PERMANOVA add-on package (Gorley and Clarke, 2006). Venn diagrams were produced in Venny online freeware (Oliveros, 2007).

\subsubsection{Phylogenetic analysis}

The first 150 OTUs contributing most to differences between groups were considered for a more thorough phylogenetic analysis. Representative sequences of these OTUs were aligned using the online SINA alignment service of the ARB-Silva database (Pruesse et al., 2012). Aligned sequences were imported into ARB in the Silva release 115 SSU NR 99 tree (Quast et al., 2013) using the ARB parsimony method without changing the tree topology. Three near neighbors of all 150 OTUs were selected according to the following criteria: (i) they were at least 800 nucleotides long and included the entire sequenced amplicon, and (ii) neighbors per OTU were picked from different published studies. An alignment containing only the neighbors was exported before constructing a Bayesian tree. Ambiguous regions of the alignment were systematically removed using the program Gblocks v.0.91b (Castresana, 2000). Default parameters were used, except allowing a minimum block length of 5 and gaps in $50 \%$ of positions. Phylogenetic trees were calculated by Bayesian analysis, using a locally installed version of MrBayes 3.2 (Ronquist et al., 2012). All parameters were treated as unknown variables with uniform prior-probability densities at the beginning of each run, and their values were estimated from the data during the analysis. All Bayesian analyses were initiated with random starting trees and were run for $10^{7}$ generations. The number of chains was set to four and Markov chains were sampled every 1000 iterations. Points prior to convergence were determined graphically and discarded. Calculated trees were imported into ARB, and short sequences obtained in this study were subsequently added by use of the ARB parsimony method without changing the tree topology. 


\subsection{Results}

\subsubsection{The effect of rearing system and time on water quality}

Analysis of environmental data showed significant differences in water quality between RAS and AS. Conductivity, $\mathrm{NO}_{3}-\mathrm{N}$ and $\mathrm{PO}_{4}-\mathrm{P}$ were higher, whereas $\mathrm{CO}_{2}, \mathrm{DO}$ and $\mathrm{pH}$ were lower in RAS $(P<0.05)$ than in AS (Table 3.1).

Table 3.1. ANOVA results with system as main factor and day as repeated measure. Average water quality parameters calculated for recirculating aquaculture system (RAS) or active suspension (AS) and for day 7 and 42 ( $n=4)$ are given. The right column gives the "System * Day" interaction $P$ values (bold for $P<0.05$ ). Within system or day, bold values indicate significant difference $(P<0.05)$. TAN: Total ammonia nitrogen; NO2-N: Nitrite nitrogen; NO3-N: Nitrate nitrogen; PO43-P: Phosphate phosphorous; CO2: Carbon dioxide; T: Temperature; DO: Dissolved oxygen; Cond.: Conductivity.

\begin{tabular}{|c|c|c|c|c|c|c|c|c|c|}
\hline \multirow{3}{*}{ Parameter } & \multicolumn{4}{|c|}{ System } & \multicolumn{4}{|c|}{ Day } & \multirow{3}{*}{ Syst*Day } \\
\hline & \multicolumn{2}{|c|}{ RAS } & \multicolumn{2}{|c|}{ AS } & \multicolumn{2}{|c|}{7} & \multicolumn{2}{|c|}{42} & \\
\hline & 7 & 42 & 7 & 42 & RAS & AS & RAS & AS & \\
\hline TAN (mg L-1) & 0.03 & 0.05 & 0.06 & 0.04 & 0.03 & 0.06 & 0.05 & 0.04 & 0.047 \\
\hline NO2 (mg L-1) & \multicolumn{2}{|c|}{0.03} & \multicolumn{2}{|c|}{0.11} & \multicolumn{2}{|c|}{0.04} & \multicolumn{2}{|c|}{0.10} & 0.303 \\
\hline NO3 (mg L-1) & 12.4 & 54.3 & 4.5 & 28.2 & 12.4 & 4.5 & 54.3 & 28.2 & 0.000 \\
\hline PO4 (mg L'-1) & \multicolumn{2}{|c|}{1.46} & \multicolumn{2}{|c|}{1.04} & \multicolumn{2}{|c|}{0.37} & \multicolumn{2}{|c|}{2.13} & 0.098 \\
\hline $\mathrm{CO} 2\left(\mathrm{mg} \mathrm{L}^{-1}\right)$ & 59.1 & 9.4 & 72.9 & 39.0 & 59.1 & 72.9 & 9.4 & 39.0 & 0.003 \\
\hline Urea $\left(\mathrm{mg} \mathrm{L}^{-1}\right)$ & \multicolumn{2}{|c|}{0.02} & \multicolumn{2}{|c|}{0.07} & \multicolumn{2}{|c|}{0.03} & \multicolumn{2}{|c|}{0.06} & 0.465 \\
\hline $\mathrm{T}\left({ }^{\circ} \mathrm{C}\right)$ & \multicolumn{2}{|c|}{27.3} & \multicolumn{2}{|c|}{27.5} & \multicolumn{2}{|c|}{27.2} & \multicolumn{2}{|c|}{27.6} & 0.884 \\
\hline pH & 8.06 & 7.28 & 8.15 & 8.10 & 8.06 & 8.15 & 7.28 & 8.10 & 0.002 \\
\hline $\mathrm{DO}\left(\mathrm{mg} \mathrm{L}^{-1}\right)$ & \multicolumn{2}{|c|}{7.72} & \multicolumn{2}{|c|}{8.00} & \multicolumn{2}{|c|}{8.18} & \multicolumn{2}{|c|}{7.55} & 0.150 \\
\hline Cond $\left(\mu \mathrm{S} \mathrm{cm}^{-1}\right)$ & 402 & 820 & 260 & 456 & 402 & 260 & 820 & 456 & 0.002 \\
\hline
\end{tabular}

Conductivity, $\mathrm{NO}_{3}-\mathrm{N}$ and $\mathrm{PO}_{4}-\mathrm{P}$ increased, and $\mathrm{CO}_{2}, \mathrm{DO}$ and $\mathrm{pH}$ dropped between day 7 and $42(\mathrm{P}<0.05)$. In contrast, temperature and concentrations of $\mathrm{NO}_{2}-\mathrm{N}$, urea and TAN were neither significantly different $(P>0.05)$ between RAS and AS, nor between day 7 and 42 .

\subsubsection{Correlation of environmental parameters with water bacterial communities}

Distance-based linear model (DistLM) analysis revealed that $\mathrm{pH}$, conductivity, $\mathrm{NO}_{3}-\mathrm{N}$ and $\mathrm{PO}_{4}^{3}-\mathrm{P}$ together explained $68 \%\left(\mathrm{R}^{2}\right.$ sequential) of the observed total variation in the composition of the bacterial community in the water. Conductivity and $\mathrm{pH}$ strongly correlated with differences in $\mathrm{BC}$ s between RAS and AS, while $\mathrm{PO}_{4}-\mathrm{P}$ and $\mathrm{NO}_{3}-\mathrm{N}$ correlated with differences in the communities between day 7 and 42 within system (Figure 3.1). The same parameters showed a highly significant system * day interaction with repeated measures ANOVA of water quality data (Table 3.1). 


\section{Distance based Redundancy Analysis (dbRDA) of water microbiota and environmental parameters}

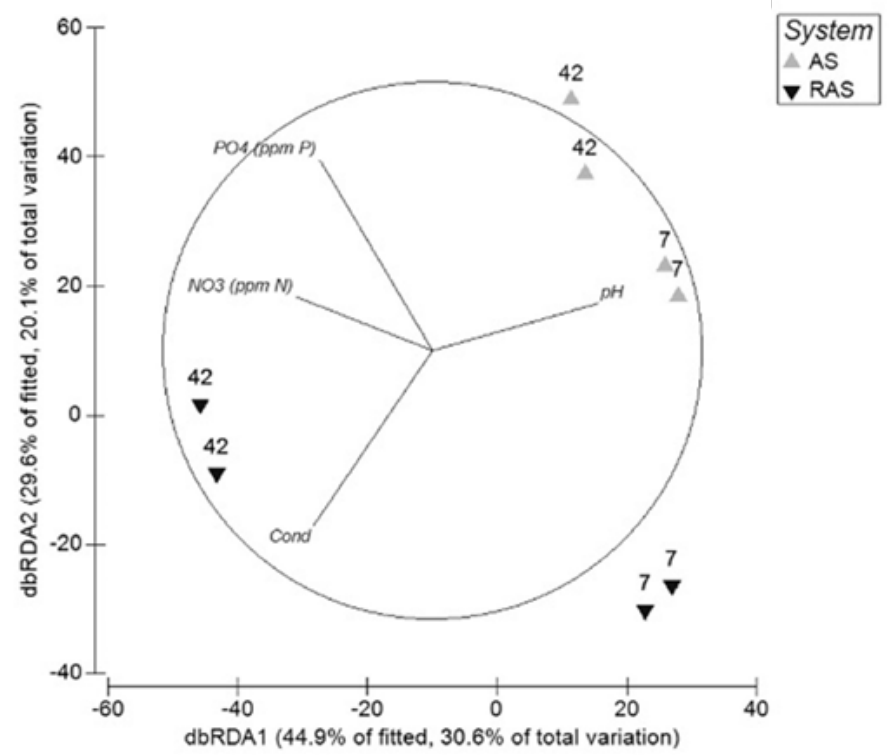

\begin{tabular}{ccccc}
\multicolumn{4}{c}{ Distance based Linear Model (DistLM) - MARGINAL TESTS } \\
Variable & SS(trace) & Pseudo-F & $P$ & $\begin{array}{c}\text { Proportion of explained } \\
\text { variation }\end{array}$ \\
$\mathrm{NO}_{3}-\mathrm{N}(\mathrm{ppm} \mathrm{N})$ & 11842 & 4.2141 & 0.0002 & 0.29647 \\
$\mathrm{PO}_{4}-\mathrm{P}(\mathrm{ppm} \mathrm{P})$ & 11195 & 3.8941 & 0.0002 & 0.28027 \\
$\mathrm{pH}$ & 12081 & 4.3359 & 0.0011 & 0.30245 \\
Cond & 11895 & 4.2408 & 0.0001 & 0.29779
\end{tabular}

res.df: 10

\section{DistLM - SEQUENTIAL TESTS (Specified solution)}

$\begin{array}{cccc}\mathrm{R}^{2} & \mathrm{RSS} & \text { No. Variables } & \text { Selection of variables } \\ 0.68073 & 12753 & 4 & \mathrm{NO}_{3}-\mathrm{N}, \mathrm{PO}_{4}-\mathrm{P}, \mathrm{pH}, \mathrm{Cond}\end{array}$

Figure 3.1. Distance based Redundancy Analysis (dbRDA) of water microbiota. Relative position of water samples in the biplot is based on Bray Curtis similarity of square root transformed relative abundance at the OTU level. Vectors indicate the weight and direction of those water quality parameters that were best predictors of water bacterial composition as suggested by the results of the distance-based linear model (distLM). The dbRDA axes describe the percentage of the fitted or total variation explained by each axis while being constrained to account for group differences. Sample IDs indicate the sampling day (7 and 42) and the rearing system (AS \& RAS: Active suspension and recirculating aquaculture system with two replicates each). $\mathrm{NO}_{3}^{-}-\mathrm{N}$ : Nitrate nitrogen; $\mathrm{PO}_{4}{ }^{3-}$ -P: Phosphate phosphorous; Cond.: Conductivity. 


\subsubsection{Similarity of water bacterial communities between replicate systems over time}

Overall, 11,658 OTUs were identified in all samples. At OTU level, the average similarity of water BC within AS on day 7 was 64\% (Table 3.2). The 5 OTUs (SIMPER analysis), in order of relative abundance, explaining most of the average similarity were OTU 9828 (order Sphingomonadales), 4552 (genus Limnohabitans), 8066 (genus Sediminibacterium), 2756 (genus Rhodobacter) and 11945 (genus Nitrospira). Combined, these 5 OTUs accounted for $49 \%$ of the bacterial community in AS water on day 7 (Table S3.1).

Table 3.2. Percentage average Bray Curtis similarity between gut, water and feed bacterial communities. Values are based on SIMPER analysis of square root transformed relative abundance data at the OTU level. Values within brackets indicate the standard deviation of the average gut Bray Curtis similarity within group, as a measure of multivariate within group dispersion. AS \& RAS: Active suspension and recirculating aquaculture systems respectively; AS1 \& AS2: Replicate active suspension system 1 \& 2; Ra \& Rb: Replicate recirculating aquaculture system a \& b; 7 and 42: Sampling day 7 and 42.

\begin{tabular}{|c|c|c|c|c|c|c|}
\hline \multicolumn{7}{|c|}{$\%$ Average Bray Curtis similarity } \\
\hline & \multicolumn{3}{|c|}{ Gut } & \multicolumn{3}{|c|}{ Water } \\
\hline & $\begin{array}{c}\text { Between } \\
\text { replicate AS } \\
(\mathrm{AS} 1, \mathrm{AS} 2)\end{array}$ & $\begin{array}{c}\text { Between } \\
\text { replicate RAS } \\
(\mathrm{Ra}, \mathrm{Rb})\end{array}$ & $\begin{array}{l}\text { Between } \\
\text { AS \& RAS }\end{array}$ & $\begin{array}{c}\text { Between } \\
\text { replicate AS } \\
(\mathrm{AS} 1, \mathrm{AS} 2)\end{array}$ & $\begin{array}{c}\text { Between } \\
\text { replicate RAS } \\
(\mathrm{Ra}, \mathrm{Rb})\end{array}$ & $\begin{array}{l}\text { Between } \\
\text { AS \& RAS }\end{array}$ \\
\hline Day 7 & $68.9( \pm 7.6)$ & $55.1( \pm 15.9)$ & 21.6 & 63.8 & 59.0 & 23.9 \\
\hline \multirow[t]{2}{*}{ Day 42} & $59.9( \pm 18.1)$ & $56.4( \pm 11.0)$ & 10.8 & 25.8 & 60.8 & 1.8 \\
\hline & \multicolumn{3}{|c|}{ Between day 7 \& 42 of gut } & \multicolumn{3}{|c|}{ Between day $7 \& 42$ of water } \\
\hline AS & \multicolumn{3}{|c|}{21.6} & \multicolumn{3}{|c|}{17.3} \\
\hline RAS & \multicolumn{3}{|c|}{27.2} & \multicolumn{3}{|c|}{3.5} \\
\hline & \multicolumn{3}{|c|}{ Day 7} & \multicolumn{3}{|c|}{ Day 42} \\
\hline & $\begin{array}{c}\text { Between } \\
\text { gut \& water }\end{array}$ & $\begin{array}{l}\text { Between } \\
\text { gut \& feed }\end{array}$ & $\begin{array}{c}\text { Between } \\
\text { water \& feed }\end{array}$ & $\begin{array}{c}\text { Between } \\
\text { gut \& water }\end{array}$ & $\begin{array}{l}\text { Between } \\
\text { gut \& feed }\end{array}$ & $\begin{array}{c}\text { Between water } \\
\text { \& feed }\end{array}$ \\
\hline AS & 4.1 & 0.1 & 0.1 & 8.5 & 0.1 & 0.1 \\
\hline RAS & 3.9 & 0.1 & 0.1 & 8.1 & 0.1 & 0.1 \\
\hline
\end{tabular}

On day 42 , the water $\mathrm{BC}$ average similarity within AS decreased to $26 \%$ (Table 3.2). Almost $60 \%$ of this similarity was attributed to OTU 8066 (genus Sediminibacterium), 14254 (family Comamonadaceae), and 4686 (order Sphingobacteriales) (Table S3.1). Other predominant OTUs of AS water BC on day 42 included: OTU 5688 (genus Fimbriimonas) and 7261 (genus Armatimonas).

Overall, water bacterial communities in AS systems on day 7 was $17 \%$ similar with BC present on day 42 since most of the predominant OTUs on one day were absent or rare on the other (Table 3.2). Despite the low similarity, 85 OTUs were shared between the two dates, with a cumulative relative abundance of $63 \%$ on day 7 and $52 \%$ on day 42 (Table 
3.3). OTU 8066 contributed most to the similarity, whereas OTU 9828 contributed most to the dissimilarity between the two days (Table S3.1).

Table 3.3. Number of unique and shared OTUs and relative abundance of shared OTUs between days. Shaded abundance values correspond to shaded sampling group in the first column. Sampling groups are compared between sampling days for each system. OTU: Operational taxonomic unit; AS \& RAS: Active suspension and recirculating aquaculture systems; $07 \& 42$ : Sampling days 7 and 42 respectively.

\begin{tabular}{|c|c|c|c|c|}
\hline \multicolumn{5}{|c|}{ Shared OTUs between days per system } \\
\hline Group & $\begin{array}{l}\text { Number of } \\
\text { shared OTUs }\end{array}$ & $\begin{array}{l}\% \text { abundance } \\
\text { of shared OTUs }\end{array}$ & $\begin{array}{l}\text { OTUs shared among } \\
\text { gut or water samples }\end{array}$ & $\begin{array}{l}\text { OTUs shared between } \\
\text { all gut \& water samples }\end{array}$ \\
\hline \multirow{2}{*}{$\begin{array}{l}\text { Gut_07_AS vs. } \\
\text { Gut_42_AS }\end{array}$} & \multirow{2}{*}{131} & 76.4 & \multirow{4}{*}{52} & \multirow{8}{*}{2} \\
\hline & & 86.4 & & \\
\hline \multirow{2}{*}{$\begin{array}{l}\text { Gut_07_RAS vs. } \\
\text { Gut_42_RAS }\end{array}$} & \multirow{2}{*}{302} & 91.0 & & \\
\hline & & 51.2 & & \\
\hline \multirow{2}{*}{$\begin{array}{l}\text { Water_07_AS vs. } \\
\text { Water_42_AS }\end{array}$} & \multirow{2}{*}{85} & 62.9 & \multirow{4}{*}{18} & \\
\hline & & 51.9 & & \\
\hline \multirow{2}{*}{$\begin{array}{c}\text { Water_07_RAS vs. } \\
\text { Water_42_RAS }\end{array}$} & \multirow{2}{*}{98} & 76.1 & & \\
\hline & & 56.8 & & \\
\hline
\end{tabular}

In RAS, the average similarity of water BC on day 7 was $59 \%$ (Table 3.2). More than half of this similarity was attributed to only two predominant OTUs: OTU 7333 (genus Polynucleobacter) and 9828 (family Sphingomonadales), which together had a cumulative relative abundance of $46.8 \%$ on that day (Table S3.1).

On day 42 , similarity of water BC within RAS remained at levels similar to day 7 . This similarity was mostly attributed to OTU 7090 (family Rhodocyclaceae), 14286 (genus Limnohabitans), and 4377 (genus Rhodobacter). Other predominant OTUs of RAS water BC on day 42 included: OTU 5688 (genus Fimbriimonas) and 7261 (genus Armatimonas). The aforementioned five OTUs accounted for $55.2 \%$ of the total bacterial community on that day (Table S3.1).

Bacterial communities in the RAS water differed considerably between day 7 and 42 (3.5\% similarity) (Table 3.2). Predominant OTUs in water on day 7 were almost absent on day 42 and vice versa. For example, OTU 7090 was absent on day 7 whilst accounted for $35 \%$ of the community on day 42 , thus contributing about $20 \%$ to the difference between the two days (Table S3.1). Despite the low similarity, water BC in RAS shared 98 OTUs between day 7 and 42 , with a cumulative relative abundance of $76 \%$ and $57 \%$, respectively (Table 3.3). 


\subsubsection{Similarity of water bacterial communities between systems}

Already on day 7, the average similarity between AS and RAS was only 24\% (Table 3.2). The two systems shared 113 OTUs with a cumulative relative abundance of $62.5 \%$ and $73 \%$ in AS and RAS systems, respectively (Table 3.4). On day 42, the similarity of water $\mathrm{BC}$ between the two systems decreased to $2 \%$, since almost all predominant OTUs in AS water, were absent in RAS and vice versa. Overall, water from the two systems shared 40 OTUs with a cumulative relative abundance of $30 \%$ in AS and only $4 \%$ in RAS (Table 3.4).

In terms of evenness, an overall system (AS \& RAS) * day (7 \& 42) interaction was observed. On day 7, 17 OTUs had a cumulative relative abundance of $60 \%$ in AS water, whereas on day 42 , the same percentage was attributed to six OTUs. In RAS water, $60 \%$ of the relative abundance was attributed to 6 OTUs on day 7 and three OTUs on day 42 (Table S3.1). Additionally, over time species richness decreased from 683 to 394 OTUs in AS water and from 815 to 552 in RAS. This observation is also in agreement with the results of the dominance plots and the biodiversity indices which showed that the richness and evenness of water bacterial communities in AS decreased between day 7 and 42, whereas this difference was less pronounced in RAS (Figure S3.2 \& Table S3.2).

Table 3.4. Number of unique and shared OTUs within and between systems. Percentages indicate the relative abundance of shared OTUs within each system. Operational taxonomic unit; AS \& RAS: Active suspension and recirculating aquaculture systems; $07 \& 42$ : Sampling days 7 and 42 respectively.

\begin{tabular}{lccc}
\hline \multicolumn{4}{c}{ Shared OTUs between systems per day } \\
\hline Group & Total number of OTUs & $\begin{array}{c}\text { Number of } \\
\text { shared OTUs }\end{array}$ & $\begin{array}{c}\text { Within group \% abundance } \\
\text { of shared OTUs }\end{array}$ \\
\hline Gut_07_AS & 732 & 247 & 70.7 \\
Gut_07_RAS & 1003 & & 71.4 \\
\hline Gut_42_AS & 279 & 90 & 43.9 \\
Gut_42_RAS & 879 & 113 & 50.7 \\
\hline Water_07_AS & 683 & 62.5 \\
Water_07_RAS & 815 & 73.0 \\
\hline Water_42_AS & 394 & 40 & 30.3 \\
Water_42_RAS & 552 & 3.6 \\
\hline
\end{tabular}

\subsubsection{Similarity of gut bacterial communities between replicate systems over time}

On day 7 , average similarity of gut bacterial communities within AS was $69 \%$ (Table 3.2). Half of this similarity was attributed to OTUs 4790 (species Mycobacterium Ilatzerense), 1651 (genus Rhodobacter), 10562 (genus Gordonia), 10599 (family Bradyrhizobiaceae) and 8299 (genus Sporocytophaga). Those five OTUs accounted for a cumulative relative abundance of $42.2 \%$ in AS (Table S3.1). On day 42, the similarity of gut BC between samples 
dropped to $60 \%$. More than $80 \%$ of this similarity was attributed to OTUs 4563 (family Isosphaeraceae), OTU 12537 (genus Arthrobacter) and OTU 4790 (species Mycobacterium llatzerense). Those predominant OTUs were encountered on both days, albeit at different relative abundances (Table 3.5). M. Ilatzerense (OTU 4790) was the only OTU with a high relative abundance on both sampling days. Despite the 131 shared OTUs between the two days, gut BC was $21.6 \%$ similar between days (Table 3.2, Table 3.3).

Within RAS, average similarity of gut BC between samples was $55 \%$ on day 7 (Table 3.2). More than $60 \%$ of this similarity was due to OTUs 10599 (family Bradyrhizobiaceae), 1005 (genus Rhodococcus), 4790 (Mycobacterium llatzerense) and 12555 (genus Agrococcus) (Table S3.1). On day 42, average similarity between gut samples remained similar. This similarity was mostly attributed to OTUs 7966 (family Peptostreptococcaceae), 4790 (Mycobacterium Ilatzerense), 7955 (family Mogibacteriaceae), 1005 (genus Rhodococcus) and 2787 (Cetobacterium somerae). These five OTUs accounted for a cumulative relative abundance of $59.5 \%$ in RAS. All predominant gut OTUs of day 7 were also present on day 42 , albeit at lower relative abundance, whereas the OTUs most predominant on day 42 were absent on day 7 (Table S3.1). Similar as for AS, M. Ilatzerense was the shared OTU with a high relative abundance on both sampling days. In RAS, 302 gut OTUs with a cumulative relative abundance of $91 \%$ and $51 \%$ respectively, were shared between days (Table 3.3).

\subsubsection{Similarity of gut bacterial communities between systems}

On day 7, similarity of gut BC between systems was 22\% (Table 3.2). Most of the predominant OTUs were present in both systems, although at different relative abundance (Table 3.5). OTUs 1651 and 8299 were predominant in AS but absent in RAS and OTU 3934 was predominant in RAS though absent in AS. Despite the low similarity in gut BC, the two systems shared 247 OTUs with a cumulative relative abundance of about $71 \%$ in both systems (Table 3.4).

On day 42 , similarity between systems decreased to $11 \%$ (Table 3.2). This was partly due to the difference in the abundance of OTU 4563 (family Isosphaeraceae) between the two systems (40.8\% in AS and absent in RAS) (Table 3.5). Among the shared OTUs on that day, $M$. Ilatzerense was the OTU with high relative abundance in both systems. Overall, the number of shared OTUs between systems decreased over time from 247 to 90 (Table 3.4).

In terms of biodiversity of the BCs, a system (AS \& RAS) * day (7 \& 42) interaction was observed. On day $7,60 \%$ of cumulative relative abundance in AS was attributed to 13 OTUs whereas on day 42 to only two; this is clearly depicted also in the large decrease of the Shannon index over time (Table S3.2). On the contrary, in RAS, the $60 \%$ of the abundance was attributed to five and six OTUs on day 7 and 42, respectively (Table S3.1) while the biodiversity indices remained on similar levels. Over time, in AS the total 
number of observed gut species decreased from 732 to 279 , whereas this decrease was not so evident in RAS. This observation is in agreement with the results of the dominance plots for day 7 (Figure S3.2).

Table 3.5. Heatmap of the most predominant gut, water and feed OTUs on day $\mathbf{7}$ and $\mathbf{4 2}$. Values represent the $\%$ average relative abundance of each OTU within each sample group and colour codes are proportional to increasing OTU abundance (from green: lower, to red: higher relative abundance). 07 and D42: sampling day 7 \& 42; AS \& RAS: Active suspension and recirculating aquaculture systems respectively.

\begin{tabular}{|c|c|c|c|c|c|c|c|c|c|c|c|c|}
\hline \multicolumn{3}{|c|}{ Taxonomy } & 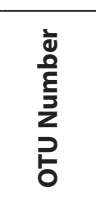 & 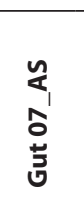 & 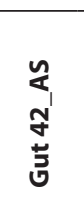 & 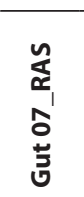 & 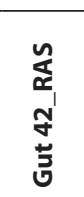 & 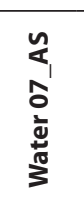 & 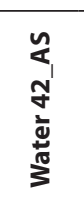 & 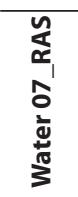 & 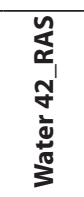 & 总 \\
\hline \multirow{6}{*}{\multicolumn{2}{|c|}{ Actinobacteria }} & M. llat & 4790 & 19.37 & 16.17 & 11.42 & 12.06 & 1.23 & 0.16 & 3.39 & 0.23 & 0.00 \\
\hline & & Gordonia & 10562 & 5.94 & 0.00 & 2.36 & 0.09 & 0.01 & 0.00 & 0.00 & 0.01 & 0.00 \\
\hline & & Rhodococcus & 1005 & 0.44 & 0.00 & 15.17 & 9.21 & 0.01 & 0.00 & 0.56 & 0.06 & 0.00 \\
\hline & & Agrococcus & 12555 & 0.01 & 0.00 & 6.80 & 0.13 & 0.00 & 0.00 & 0.03 & 0.00 & 0.00 \\
\hline & & Corynebacterium & 5872 & 0.01 & 0.00 & 0.00 & 0.00 & 0.00 & 0.00 & 0.00 & 0.00 & 12.34 \\
\hline & & Arthrobacter & 12537 & 1.17 & 19.29 & 0.00 & 0.01 & 0.07 & 0.36 & 0.00 & 0.00 & 0.00 \\
\hline \multirow{2}{*}{\multicolumn{2}{|c|}{ Bacteroidetes }} & Sporoc & 8299 & 3.80 & 0.00 & 0.00 & 0.00 & 0.06 & 0.00 & 0.00 & 0.00 & 0.00 \\
\hline & & Sediminibacterium & 8066 & 0.00 & 0.00 & 0.00 & 0.00 & 4.29 & 20.30 & 0.01 & 0.07 & 0.00 \\
\hline \multirow{4}{*}{\multicolumn{2}{|c|}{ Firmicutes }} & Peptostreptococcaceae & 7966 & 0.44 & 0.04 & 0.00 & 18.13 & 0.01 & 0.00 & 0.00 & 1.70 & 0.00 \\
\hline & & Kestanbolensis & 1521 & 0.00 & 0.00 & 0.00 & 0.03 & 0.00 & 0.00 & 0.00 & 0.00 & 4.12 \\
\hline & & Mogibacteriaceae & 7955 & 0.02 & 0.01 & 0.00 & 8.40 & 0.00 & 0.00 & 0.00 & 0.26 & 0.00 \\
\hline & & Facklamia & 3532 & 0.00 & 0.00 & 0.00 & 0.00 & 0.00 & 0.00 & 0.00 & 0.00 & 3.94 \\
\hline \multicolumn{2}{|c|}{ Fusobacteria } & Psychrilyobacter & 2650 & 0.00 & 0.00 & 0.00 & 0.00 & 0.00 & 0.00 & 0.00 & 0.00 & 7.11 \\
\hline \multicolumn{2}{|c|}{ Nitrospirae } & Nitrospira & 11945 & 0.00 & 0.00 & 0.01 & 0.01 & 4.10 & 0.77 & 0.18 & 0.01 & 0.00 \\
\hline \multicolumn{2}{|c|}{ Planctomycetes } & Isosphaeraceae & 4563 & 3.27 & 40.75 & 0.00 & 0.00 & 0.03 & 1.95 & 0.00 & 0.00 & 0.00 \\
\hline \multirow{11}{*}{ 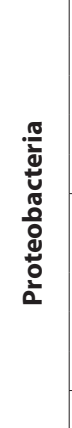 } & \multirow{5}{*}{ Alpha } & Bradyrhizobiaceae & 10599 & 3.78 & 0.27 & 18.92 & 0.63 & 0.08 & 0.02 & 0.66 & 0.02 & 0.00 \\
\hline & & Sphingomonadales & 9828 & 0.00 & 0.00 & 0.00 & 0.00 & 18.30 & 0.09 & 15.66 & 0.16 & 0.00 \\
\hline & & Rhodospirillaceae & 4701 & 0.00 & 0.01 & 0.00 & 0.00 & 0.05 & 1.40 & 0.03 & 10.73 & 0.00 \\
\hline & & Rhodobacter & 1651 & 9.35 & 0.05 & 0.00 & 0.05 & 1.10 & 0.01 & 0.00 & 0.00 & 0.00 \\
\hline & & Rhodobacter & 4377 & 0.00 & 0.00 & 0.00 & 0.00 & 0.00 & 0.00 & 0.00 & 12.55 & 0.00 \\
\hline & \multirow{5}{*}{ Beta } & Limnohabitans & 4552 & 0.01 & 0.00 & 0.00 & 0.00 & 7.03 & 3.00 & 0.04 & 0.01 & 0.00 \\
\hline & & Polynucleobacter & 7333 & 0.00 & 0.00 & 0.00 & 0.00 & 0.33 & 0.40 & 31.16 & 0.11 & 0.00 \\
\hline & & Comamonadaceae & 14254 & 0.00 & 0.03 & 0.00 & 0.00 & 0.07 & 5.25 & 0.00 & 0.00 & 0.00 \\
\hline & & Rhodocyclaceae & 7090 & 0.00 & 0.00 & 0.00 & 0.00 & 0.00 & 0.01 & 0.00 & 35.63 & 0.00 \\
\hline & & Limno & 14286 & 0.00 & 0.00 & 0.00 & 0.00 & 0.04 & 0.01 & 0.03 & 10.73 & 0.00 \\
\hline & Gamma & Photobacterium & 14212 & 0.00 & 0.00 & 0.00 & 0.00 & 0.00 & 0.00 & 0.00 & 0.00 & 10.90 \\
\hline
\end{tabular}

\subsubsection{Predominant OTUs of feed bacterial communities}

Since only one feed sample was analyzed per sampling day, no statistical comparison between sampling days was performed. OTUs 5872 (genus Corynebacterium), 14212 (genus Photobacterium), 3747 (genus Sporosarcina), 2650 (genus Psychrilyobacter), 3532 (genus Facklamia) and 1521 (species Anoxybacillus kestanbolensis), were among the most abundant OTUs on both days (Table S3.1, Table 3.5). 


\subsubsection{Relationship between gut, water and feed bacterial communities}

RELATE analysis revealed a strong correlation (Spearman rank correlation Rho 0.807, P $<0.0001$ ) between bacterial communities in gut and water (Table S3.3). In other words, differences among water and gut samples followed similar patterns, even though the bacterial community composition in gut and water was different $(\mathrm{P}<0.001)$. On day 7 , Proteobacteria dominated the water of both systems. In RAS, Proteobacteria was also the dominant phylum on day 42, but Fusobacteria and Firmicutes, which were absent on day 7, appeared. In AS systems, Bacteriodetes and Armatimonadetes replaced a large fraction of the Proteobacteria by day 42. Feed-associated BC was dominated by Firmicutes and Proteobacteria, followed by Actinobacteria and Fusobacteria (Figure 3.2).

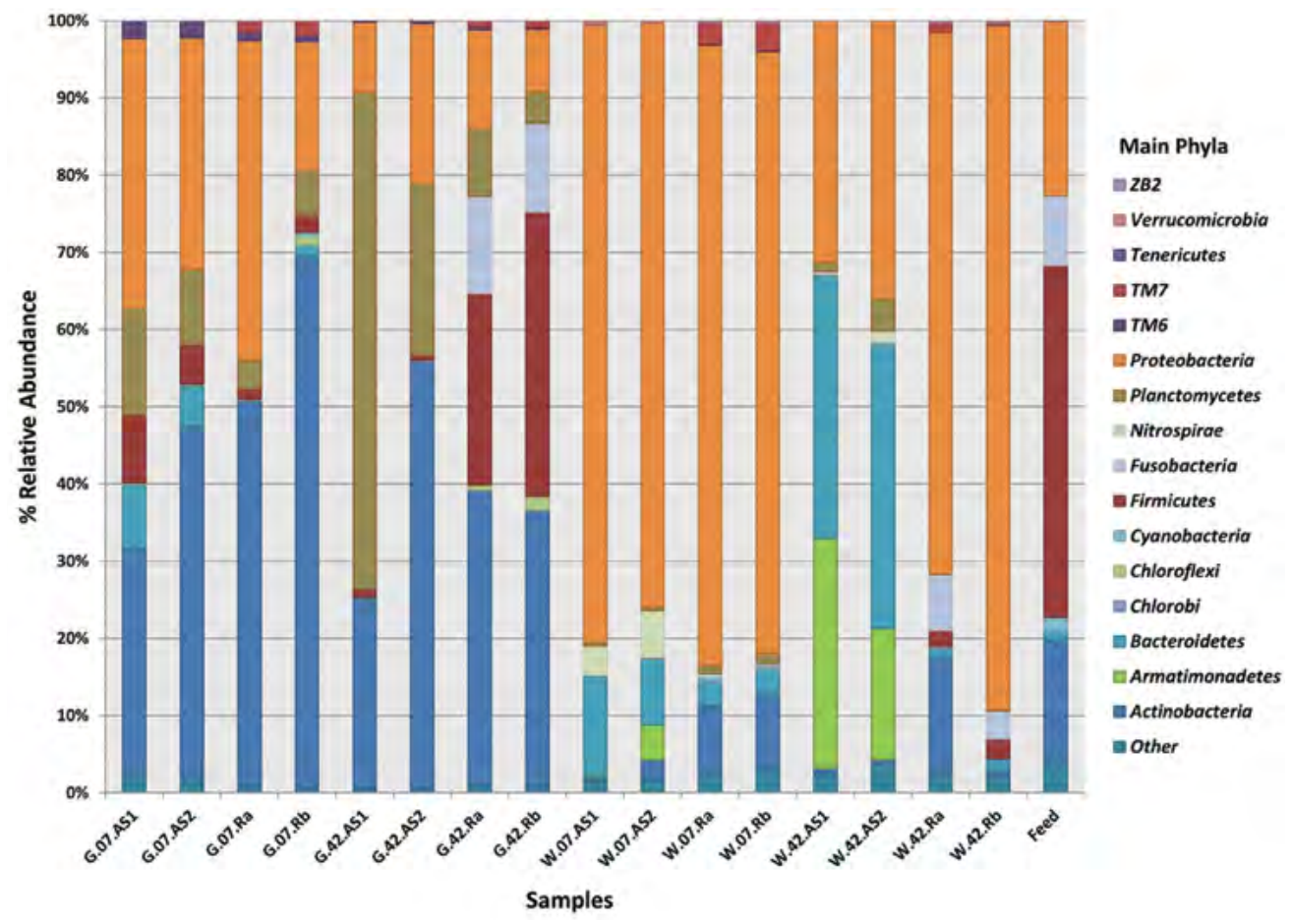

Figure 3.2. Cumulative bar charts of the main phyla present in either gut or water samples. Percentages show the relative abundance of each phylum in the gut or water of each replicate system on either day 7 or 42 . Phyla in the feed represent the average values of feed samples from both day 7 and 42. AS1 \& AS2: Replicate active suspension system 1 and 2, Ra \& Rb: Recirculating aquaculture system $a$ and $b$.

In spite of a high inter-sample correlation, average similarity between gut and water bacterial communities in AS was between $4 \%$ and $8 \%$ for both days (Table 3.2). This was mostly due to the low number of subdominant OTUs shared between gut and water (Figure 3.3). This low similarity between gut and water on day 7 was attributed to 41 
shared OTUs which represented $50 \%$ of the cumulative relative abundance in gut and $29 \%$ in water. On day 42,40 shared OTUs between gut and water had a cumulative relative abundance of $90 \%$ and $40 \%$, respectively (Table 3.6 ).

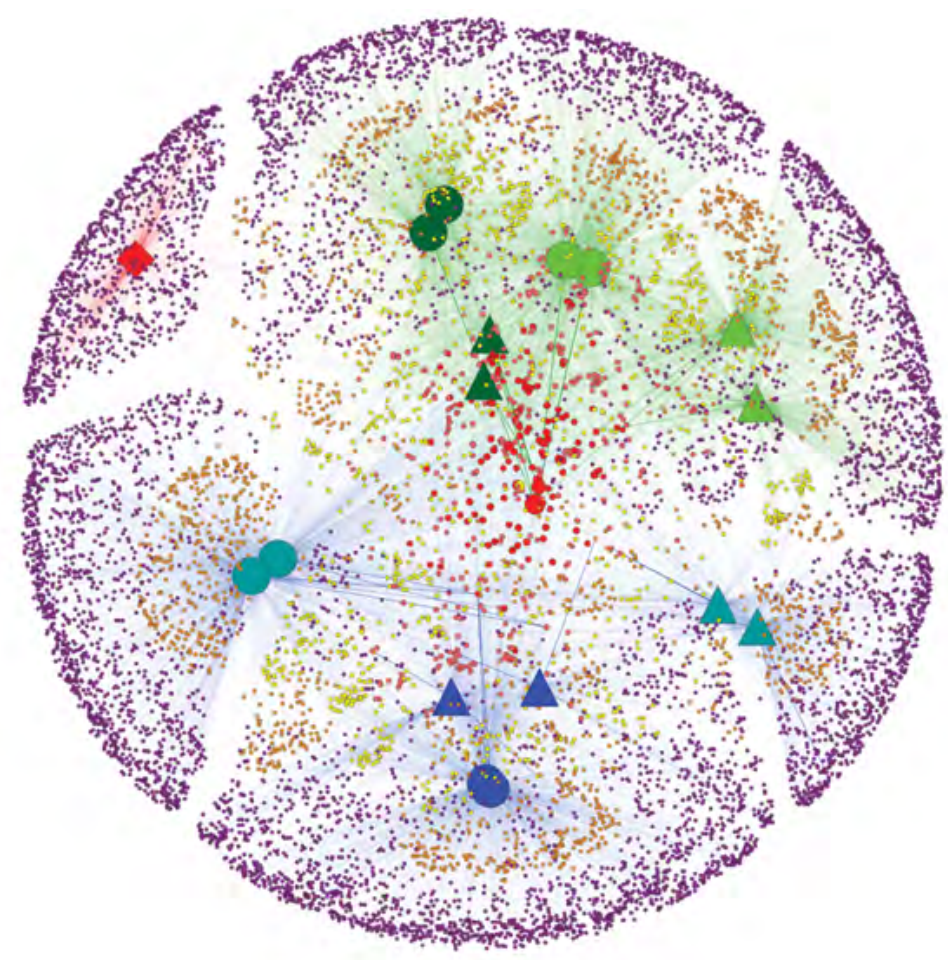

Figure 3.3. Network-based analyses of gut, water and feed bacterial communities. The network diagram is colorcoded by: sample type (gut: green, water: blue and feed: red); day 07 (circles), day 42 (triangles) and feed which represents a pooled sample both from day 7 and 42 (square); system (gut AS: Active suspension system with two replicates: dark green; gut RAS: Recirculating aquaculture system with two replicates: light green; water AS: Active suspension system with two replicates: dark blue; water RAS: Recirculating aquaculture system with two replicates: light blue). Sample nodes are oversized to be distinguished from OTU nodes. Colour and size of OTU nodes represent assignments of OTUs to sample nodes based on the amount of samples that were shared with (small nodes are shared with less samples compared to larger ones); purple OTU nodes: OTUs present in only one sample; orange OTU nodes: OTUs shared between two samples; yellow OTU nodes: shared between 3 or 4 samples; pink OTU nodes: shared between 5 to 7 samples; red OTU nodes: shared between 8 to 16 samples). Thickness/brightness of edges connecting two nodes is proportional to the relative abundance of the specific OTU in the physical sample to which it connects.

In RAS, similarity between gut and water BC was 4 and $8 \%$ in the two day respectively (Table 3.2). Similarity on day 7 was attributed to 155 shared OTUs with a cumulative relative abundance of $83.5 \%$ and $20 \%$, in gut and water respectively. On day 42 , the 86 shared OTUs had a cumulative relative abundance of $81 \%$ and $12 \%$ (Table 3.6). 
On the two days, gut shared with feed ten and five OTUs in AS and eight and three in RAS (Table 3.6). For both systems and days, the similarity between gut and feed was below 1\% (Table 3.2). In AS, OTU 5872 was the only dominant OTU in feed encountered in the gut on day 7. Overall, only two OTUs were shared among gut, water and feed bacterial communities in AS or RAS on day 7, and only one OTU on day 42 (Table 3.6).

Table 3.6 Total number and percentage relative abundance of shared OTUs per system on each sampling day. Shaded relative abundance values correspond to shaded sampling group in the first column. Sample types are compared within system for each days. OTU: Operational taxonomic unit; AS \& RAS: Active suspension and recirculating aquaculture systems; $07 \& 42$ : Sampling days 7 and 42 respectively.

\begin{tabular}{|c|c|c|c|c|c|c|c|c|c|c|}
\hline \multirow{2}{*}{ Group } & \multicolumn{10}{|c|}{ Number and \% abundance of shared OTUs } \\
\hline & \multicolumn{3}{|c|}{ 07_AS } & \multicolumn{2}{|c|}{ 07_RAS } & \multicolumn{2}{|c|}{ 42_AS } & \multicolumn{3}{|c|}{ 42_RAS } \\
\hline \multirow{2}{*}{ Gut vs. Water } & \multicolumn{3}{|c|}{41} & \multicolumn{2}{|c|}{155} & \multicolumn{2}{|c|}{40} & \multicolumn{3}{|c|}{86} \\
\hline & 50.0 & & 28.6 & 83.5 & 20.2 & & 39.7 & 81. & & 12.1 \\
\hline \multirow{2}{*}{ Gut vs. Feed } & \multicolumn{3}{|c|}{10} & \multicolumn{2}{|c|}{8} & \multicolumn{2}{|c|}{5} & \multicolumn{3}{|c|}{3} \\
\hline & 0.2 & & 18.6 & 1.1 & 2.5 & & 1.1 & 0.0 & & 0.8 \\
\hline \multirow{2}{*}{ Water vs. Feed } & \multicolumn{3}{|c|}{3} & \multicolumn{2}{|c|}{3} & \multicolumn{2}{|c|}{3} & \multicolumn{3}{|c|}{3} \\
\hline & 3.2 & & 0.1 & 2.4 & 0.1 & 0.3 & 1 & & 2 & 0.1 \\
\hline \multirow{2}{*}{ Gut vs. Water vs. Feed } & \multicolumn{3}{|c|}{2} & \multicolumn{2}{|c|}{2} & \multicolumn{2}{|c|}{1} & \multicolumn{3}{|c|}{1} \\
\hline & 0.1 & 2.2 & 0.1 & 0.9 & 0.1 & 0.0 & 0.1 & 0.0 & 0.1 & 0.1 \\
\hline
\end{tabular}

\subsection{Discussion}

The higher levels of nitrate observed in RAS compared to AS could be due to the higher nitrification rates. In RAS, through the water re-use loop, settable solids consisting mainly of organic matter, are removed before aerobic chemoautotrophic nitrifying bacteria convert ammonia via nitrite into nitrate in the biofilter (Crab et al., 2007; Gutierrez-Wing and Malone, 2006; Schreier et al., 2010). In AS, solids were not removed from the fish rearing unit, but maintained in suspension. The accumulating organic matter in AS was used as substrate for biofloc formation (Avnimelech, 2007; Azim and Little, 2008; De Schryver et al., 2008; Hargreaves, 2006). Heterotrophic bacteria in bioflocs immobilize $\mathrm{NH}_{3}-\mathrm{N}$, reducing the need for nitrification. Nitrification concurs with reduction of alkalinity. This explains why in AS the $\mathrm{pH}$ was higher than in RAS. However, the lower $\mathrm{pH}$ in RAS did not result in a higher $\mathrm{CO}_{2}$ level, because the amount of free $\mathrm{CO}_{2}$ in a system depends on the total amount of carbon dioxide/bicarbonate/carbonate (i.e. the total alkalinity) present (Ebeling et al., 2006).

In AS systems, a nitrification-denitrification coupling might have contributed to the observed lower $\mathrm{NO}_{3}-\mathrm{N}$ concentrations (da Silva et al., 2013; Ray et al., 2011). Nitrification occurs in the aerobic surface layer of bioflocs, whereas denitrification can take place in deeper anoxic layers (de Kreuk et al., 2005; Satoh et al., 2003; Third et al., 2003). The 
high relative abundance of Nitrospira in water bacterial communities of AS on both days, suggests the occurrence of nitrification in those systems. Nitrospira is an aerobic, lithoautotrophic, nitrite-oxidizing bacterium that is commonly encountered in aquaculture systems or in freshwater activated sludge particles (Mußmann et al., 2013). However, this genus exhibits high substrate affinity and has been mainly reported in filter materials, biofilters of freshwater, brackish and marine systems (Sugita et al., 2005; Kruse et al., 2013; Schreier et al., 2010; Itoi et al., 2007; Brown et al., 2013).

The second most abundant OTU (14254) in AS water of day 42 belonged to the Comamonadaceae family. Members of this family play a role in nitrate removal as well as in poly-hydroxy-alkanoate (PHA) metabolism. This indicates the metabolic and regulatory relationships between PHA degradation and denitrification by PHA-degrading denitrifiers in anoxic environments (Khan et al., 2002; Ginige et al., 2005; Sadaie et al., 2007; Adav et al., 2010a).

The $\mathrm{PO}_{4}-\mathrm{P}$ concentration in RAS has been higher than in AS. Microbial phosphate assimilation was suggested as the main cause of the lower phosphate levels in biofloc systems when compared to those in RAS (Brito et al., 2013; da Silva et al., 2013; Luo et al., 2014). The most predominant OTU in RAS water on day 42 was a member of family Rhodocyclaceae. This family contains mainly aerobic or denitrifying bacteria which live in aquatic habitats and exhibit very versatile metabolic capabilities. Rhodocyclaceae species are commonly reported in waste water where they play an important role in bioremediation (e.g. phosphate removal) (Quan et al., 2006; Fernández et al., 2009; Tsai et al., 2005; Ebrahimi et al., 2010; Adav et al., 2010b; Guo et al., 2011). Information at genus level on its role in phosphorus cycling would be needed to explain differences in abundance between AS and RAS.

DistLM analysis revealed a high correlation between water quality parameters and the bacterial communities present in the water. A high fraction of the total variation $(68 \%)$ in water $\mathrm{BC}$ was explained by $\mathrm{pH}, \mathrm{NO}_{3}-\mathrm{N}, \mathrm{PO}_{4}-\mathrm{P}$ and conductivity. Ebeling et al. 2006, described under which conditions autotrophic, heterotrophic, nitrifying or denitrifying processes dominate in aquatic systems (Ebeling et al., 2006). In fact, differences in water quality between RAS and AS were caused by differences in system design and management, which induced differences in composition and functionality of water $\mathrm{BC}$ and vice versa. Although the percentage of explained variation in water BC by environmental factors was high, it remains challenging to control bacterial communities composition, species dynamics and functionality because: (a) possibly other factors, presently not measured, are better "predictors" of the bacterial communities and (b) there is a high degree of stochasticity involved in how bacterial communities structures change over time, even in replicated systems (Giatsis et al., 2014). In addition, interpretation of the DISTLM results should be done with care, especially when sample size is low. Despite the fact that permutation 
methods allowed us to reach the desirable significance level to test our hypothesis (i.e. correlation between water quality and water microbial communities), the high percentage of explained variation does not directly imply causality between the two datasets, but correlation. Future research, including more systems and data points should be performed in order to verify if the observed correlations are maintained.

The time * system interaction for water $\mathrm{BC}$ revealed very interesting trends. First, the similarity of water BC between replicate AS tanks decreased over time, whereas in RAS it remained stable. This suggests that sustainable "replication of water bacterial communities" is more feasible in RAS than in AS. Secondly, the average similarity of water BC between day 7 and 42 in AS was higher than in RAS. Although in RAS more OTUs were shared between days, water bacterial communities in AS remained five times more similar over time compared to that in RAS. This observation could be partly explained by some predominant OTUs in the water, which over time, were more robust in AS than in RAS. For example, Sediminibacterium (OTU 8066) in AS water was among the most predominant OTUs on both days. This genus contains two species exhibiting high versatility in types of used substrates including nonstarch polysaccharides (NSPs), oligosaccharides and others. These species were found in lakes, freshwater reservoirs, activated sludge reactors, stream biofilms and nutrient rich environments (Kim et al., 2013; Kahlisch et al., 2012; Torrentó et al., 2011; Wang et al., 2012; Singleton et al., 2011; Khemkhao et al., 2011; Besemer et al., 2012).

Water BCs similarity between AS and RAS systems decreased considerably over time, as shown by the noticeable (from 247 to 90 ) decrease of the number of shared OTUs (Table 3.4). On day 7, the predominant water OTUs of either AS or RAS were found in both systems though in different relative abundances. On day 42, the predominant OTUs in water of AS systems were not encountered in RAS, and vice versa. Both systems were initially inoculated with water from the same source, and thus with a similar BC, which shows that the system design and function induced the development of a system-specific specialized bacterial community.

It is important at this point to remember that regarding water BCs in particular, the observations and patterns discovered between the two systems, or over time, are just a useful first starting point. This is firstly because in some cases sample size was low (especially for within system comparisons), though high enough to allow statistical power. Secondly, the environments in this study, although relevant to aquaculture are not natural since they operate under controlled conditions. This means that it is uncertain whether the observed patterns would occur in natural habitats or for example in an extensive outdoor aquaculture system (e.g. a fish pond). Nevertheless, this work can be the basis of future work addressing the implications that rearing systems have on water and fish gut BCs and provide awareness on system microbial management in aquaculture. 
The described differences in composition of water BC between the two systems give rise to the question whether these differences had an impact on gut communities, and of course to which extent. The assumption has been that water provides the first microbial colonizers from the moment of mouth opening until first feeding, whereas feed microbiota would dominate during later development (Reitan et al., 1998; Ringo and Birkbeck, 1999; Hansen and Olafsen, 1999). Bakke et al. (2013), suggested that relatively small differences in water BC might impose significant differences in gut BC of fish larvae (Bakke et al., 2013). In line with this suggestion, our results showed a high and consistent correlation between the bacterial community composition in water and gut (Spearman's $\rho: 0.807)$.

Although the similarity of water BC between replicate AS decreased over time, the average similarity of gut BC decreased little. Similarly in RAS, a significant decrease in average similarity of water $B C$ over time was not observed in gut which remained eight times higher in gut than in water (Table 3.2). A considerable number of gut OTUs was shared between day 7 and 42. For example in RAS, OTU 1005 (genus Rhodococcus) was a predominant OTU in the gut on both days. The genus Rhodococcus has been reported in gut microbiota of sole, red rock fish, Norwegian mackerel, USA smelt, rainbow trout and shrimp (Kim et al., 2007; Sanchez et al., 2012; Spanggaard et al., 2000; Huber et al., 2004).

The relatively larger effect of time on water communities suggests that water BC were more sensitive to environmental changes than bacterial communities residing in the gut of fish larvae. To a certain degree, microbial community stability highly depends upon potential disturbances of the two ecosystems i.e. the gut and the water, as well as the ability of a community to respond resiliently. Preservation of homeostasis at the intestine should be in the gut microbiota's best interest, in order to provide a convenient longterm habitat. In favor of both, host also contributes to a fairly stable environment leading to a homeostatic interaction between host and gut microbiota. However, during early fish development, rapid anatomical, physiological and immunological changes occur in the gut, which makes it plausible to think that equally substantial changes should have also occurred in the gut communities. Fernandez et. al (2000), evaluated the behavior of different microbial communities under perturbed conditions and suggested that stability of the functionality does not necessarily imply stability of community structures. This means that in our study, observed changes in the gut community structure over time, could have occurred side-by-side with changes in community functionality. In the same line of thought, observed changes of water physicochemical properties might have been responsible for the relative change of water BCs over time, but whether the latter compromised functional stability of the community, is not known. Further research is required to elucidate the relationship between microbial community structure (i.e. composition, diversity) and its ability to organize in a way that assures the ability of counteracting the stress effects, by maintaining functionality. 
Regarding predominant species, an interesting system * day interaction was observed in the gut communities. On day 7, the number of observed gut species in the AS systems was higher than in RAS. This higher richness coincided with higher gut community evenness i.e. higher biodiversity. On day 42 a considerable decrease both in the community richness and evenness was observed in AS. The latter means that a smaller fraction of the different species was present in dominant numbers whereas this was not so evident for fish reared in the RAS systems. Based on our data, it is not possible to clarify with certainty the reason for the observed differences between the two systems. Nevertheless, the fact that similar temporal changes in community biodiversity were also observed in water BCs might point out the role of water as a microbial reservoir available to the fish gut. However, more studies would be necessary to test how changes in community structure of a habitat impact gut microbial biodiversity of the organisms living therein and whether such changes affect the functionality of the gut community.

Similarity of gut bacterial communities between AS and RAS was overall low and further decreased over time. On day 7, OTU 1651 (genus Rhodobacter) was a predominant OTUs in the gut of AS, but absent in RAS. The genus Rhodobacter contains Gram-negative bacteria widely distributed in fresh water as well as marine and hypersaline habitats (Table S3.4, Figure S3.3). Rhodobacter species have been reported in the gut of grass carp, rainbow trout, Siberian sturgeon, catfish, Atlantic cod and Pacific white shrimp (Wu et al., 2012b; Wong et al., 2013; Mansfield et al., 2010; Geraylou et al., 2013; Di Maiuta et al., 2013; Fjellheim et al., 2011; Zhang et al., 2014), as well as in the water of aquaculture systems (Tang et al., 2014; Rurangwa and Verdegem, 2014).

A similar pattern was observed for the gut BC in RAS. OTU 7966, (family Peptostreptococcaceae) was predominant in the gut on day 42 , absent on day 7 , and present at very low relative abundance levels in AS. This suggests that both systems and developmental stage of the fish influence the presence and abundance of this OTU. Peptostreptococcaceae is a family of obligate anaerobic bacteria (Ringø et al., 1995). In salmon, Peptostreptococcaceae was detected as a member of both the allochthonous and autochthonous microbiota regardless of the fish diet, indicating that Peptostreptococcaceae might be a natural part of the intestinal microbiota of this species (Hartviksen et al., 2014). The family has been also reported in the gut microbiota of yellow catfish (Wu et al., 2012a, 2010) and common carp (van Kessel et al., 2011).

In this study, out of 11,658 OTUs, two OTUs were detected in all gut and water samples. Of these, Mycobacterium llatzerense, a member of the phylum Actinobacteria, was present at high relative abundance in all gut samples, and less abundant in water samples. The second OTU, a member of the Bradyrhizobiaceae, was among the predominant gut OTUs on day 7 for both systems, less abundant on day 42 , and also present in all water samples. Whether its presence in the water was due to fish defecation or whether it originated from water and managed to proliferate in the fish gut is presently not known. 
M. llatzerense, has not been previously reported in aquaculture systems but has been reported in hemodialysis water, and drinking water production and distribution systems (Dubrou et al., 2013). The association of M. llatzerense with groundwater (Table S3.4, Figure S3.3) may reflect a more psychrophilic profile of this species compared to other Mycobacteria, and might justify its presence in our systems which rely on well water. Although M. Ilatzerense has not been previously reported in fish gut microbiota, the high and consistent abundance in all guts suggests the preference of these bacteria for the gut environment of tilapia larvae. M. Ilatzerense is facultative autotrophic, aerobic, non-fermentative, and involved in hydrogen oxidation and alkaline dephosphorylation (Gomila et al., 2008).

Members of the Bradyrhizobiaceae family have been found in sewage treatment plants (Zhang et al., 2012), municipal wastewater treatment plants (Ye and Zhang, 2013), active granular activated carbon filters and drinking water distribution systems (Table S3.4, Figure S3.3) (Liu et al., 2012b). This is in agreement with the presence of Bradyrhizobiaceae in all water samples of this study. In fish, the Bradyrhizobiaceae family was overrepresented in the gut microbiota of sea bass (Carda-Diéguez et al., 2014), and was also reported in the stomach of yellow catfish (Wu et al., 2012a). Since the family contains more than ten different genera, it is difficult to speculate about the role and functionality of these microorganisms in the fish gut.

More information and relevant literature regarding major functions and processes as well as environment preference of the encountered OTUs are provided in Table S3.4 of the supplementary material.

Interestingly, the OTUs shared between gut and water had a higher relative abundance in the gut than in the water. In turn, almost none of the dominant OTUs in water were detected in the gut. This could suggest that host-specificity for particular microbial species is modulated by selective pressures within the host gut attributed to gut habitat (i.e. physiology, anatomy) and host's genotype (Yan et al., 2012; Rawls et al., 2006; Navarrete et al., 2012). On the other hand in water, low abundance of the predominant gut OTUs can be partly explained by fish defecation. Apparently, conditions in water are suboptimal for the growth of defecated bacteria mostly due to the ecological preference of the latter for the gut habitat (i.e. pH, anoxic conditions, etc.), adhesion sites and nutrients availability therein. Despite the significant effort in defining the forces which impact the assembly of gut microbial communities, the underlying mechanisms attributed to the impact of the environment over the host selectivity are still poorly understood.

Feed predominant bacteria were mostly absent in water and gut. Only $1 \%$ of gut OTUs was shared with feed at very low relative abundances. It is important at this point to mention that these results do not underestimate the importance of feed per se on gut microbial communities. Feed composition, nutrients, production technology, etc. have been all found to affect gut microbiota in mammals (Scott et al., 2011; Flint et al., 2007; Walker et al., 
2011; Turnbaugh et al., 2009b; Muegge et al., 2011; Delsuc et al., 2014) and fish (Merrifield et al., 2011; Ingerslev et al., 2014; Meziti et al., 2012; Hartviksen et al., 2014). This is not the case in our study where the same feed was used in all treatments. We put forward that the low number of subdominant bacteria shared between the feed and the gut indicates the low influence that feed communities had in the gut compared with those present in the water. Bakke et al. 2013, working with cod larvae, suggested that microbiota in water had a stronger influence than microbiota in the feed on the microbial community composition in the gut (Bakke et al., 2013). This was clearly pictured in our OTU network (Figure 3.3) where only a few OTUs present in feed were also present in water or gut samples. Corynebacterium (OTU 5873) was an exception since it was the predominant OTU in feed but also present in the gut of fish larvae in AS on day 7. Corynebacteria are found in a broad variety of habitats such as soil, plants and food products (Yassin et al., 2003).

\section{Shared and unique OTUs between gut, water \& feed microbiota}
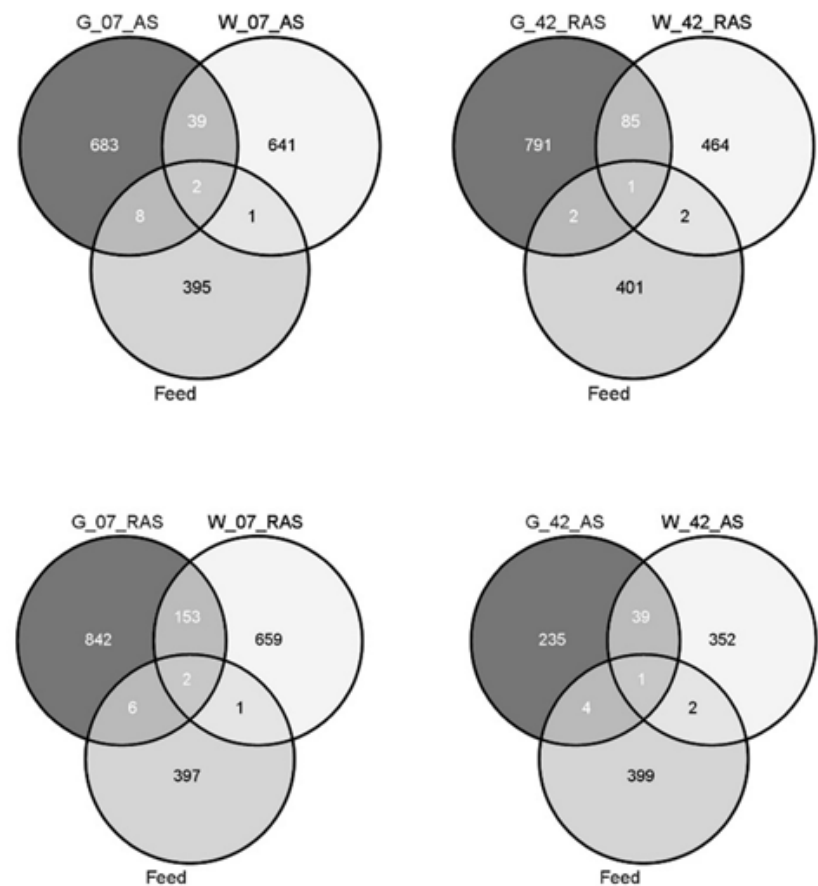

Figure 3.4. Venn diagrams of bacterial communities between sample types. Number of reads in all samples was normalized to the minimum number of reads found in one of the samples (1719 reads). The Venn diagram illustrates overlap of bacterial OTUs between gut, water and feed samples for two different systems over time. Numbers inside circles indicate the number of unique OTUs in one of the subsets based on absence/presence data from replicate systems. Numbers of unique OTUs indicate those OTUs that were present in both replicate systems and in at least one of the samples within each replicate system. Numbers in the overlapping regions indicate the number of shared OTUs between subsets. AS \& RAS: active suspension and recirculating aquaculture systems; 07 and 42: Sampling day 7 and 42 respectively. 
The low average similarity and the small number of OTUs shared between gut, water and feed (Table 3.2, Figure 3.4) suggest that the microbial community in the gut is not a simple reflection of the microbes from the environment. In our study, bacterial communities in rearing water influenced those present in the gut; nevertheless they only had a limited effect on the predominant gut species. Factors affecting community development include: suitability of adhesion sites and substrates availability, host physiology, gut anatomy and abiotic components in different sections of the gut, as well as the host's non-specific and specific immunity (Clements et al., 2014; Hansen and Olafsen, 1999). Thus, the resulting gut microbial community can be very different from the microbiota in water or feed. Sullam et al. (2012) stated that fish hindgut microbial communities resemble much more those of mammals, than those present in water or feed. Our study confirms that fish, like other vertebrates, harbor specialized gastrointestinal communities which are a skewed selection of microbes present in water and feed.

\subsection{Conclusion}

The culture system strongly affected the composition and development of bacterial communities in water. Despite low similarities between gut and water, observed changes in water bacterial communities were highly correlated with changes in the gut. However, there is no clear evidence that bacteria in the water from one system had a larger impact on gut communities than the other. The potential role of the few shared or discriminant species explaining a fraction of the observed similarity was also evaluated. Most of the predominant or shared OTUs in the fish gut have also been found in other fish species, which suggests that these microorganisms fulfil similar roles in the gut of different species. By studying the roles and functionalities of the shared species, novel insights on their niche and ecological adaptability of microbiota in water and gut, and to a lesser extend feed, will be generated. A better understanding of how microbiota in water, feed and gut interact will help improving the design, microbial management and nutrient cycling of fish rearing systems. Future research should focus on disentangling the key processes governing selection and propagation of water and feed microbiota, which ultimately become important species of the microbial community in the adult fish gut.

\section{Acknowledgements}

This work was funded by The European Community's Seventh Framework Program (FP7/2007-2013) under grant agreement no. 227197 Promicrobe “Microbes as positive actors for more sustainable aquaculture". 


\section{Supplementary material}

Table S3.1. Similarity percentages (SIMPER) indicating the most abundant OTUs contributing to the discrimination between groups, or to the relatedness of samples within a group. Average similarity: Overall Bray Curtis similarity among samples within the same group; Average dissimilarity: Overall Bray Curtis dissimilarity between samples of the two groups in comparison; Av. Abundance: Average relative abundance of an OTU within a group; Av. Sim.: Contribution of an OTU to the average group similarity; Av. Diss.: Contribution of an OTU to the average dissimilarity between the two groups in comparison; Sim/SD: Ratio of the average contribution of an OTU to the average similarity within the group, divided by the SD (in the case of AS water microbiota, \# indicates that no value was calculated due to the lack of replication and estimation of the SD) ; Diss./SD: Ratio of the average contribution of an OTU to the average dissimilarity within the group, divided by the SD (low SD suggests consistency of a discriminant OTU); Contrib\%: Percentage contribution of an OTU to the similarity within a group or to the dissimilarity between groups in comparison; Cum.\%: Cumulative percentage contribution of OTU's based on column Contrib\%; OTU: Operational taxonomic unit No; AS \& RAS: Active suspension and recirculating aquaculture systems respectively; 7 and 42: Sampling day 7 and 42.

\section{Type: Gut, Day: 7, System: AS}

Average similarity: 68.91

\begin{tabular}{|c|c|c|c|c|c|c|}
\hline Taxonomic ID & 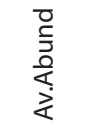 & 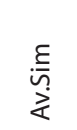 & 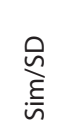 & 命 & $\stackrel{\circ}{\stackrel{\circ}{\xi}}$ & $\stackrel{?}{b^{\prime}}$ \\
\hline $\begin{array}{l}\text { Bacteria Actinobacteria Actinobacteria Actinomycetales } \\
\text { Mycobacteriaceae Mycobacterium llatzerense }\end{array}$ & 19.37 & 13.68 & 1.83 & 19.85 & 19.85 & 4790 \\
\hline $\begin{array}{l}\text { Bacteria Proteobacteria Alphaproteobacteria } \\
\text { Rhodobacterales Rhodobacteraceae Rhodobacter }\end{array}$ & 9.35 & 7.01 & 3.97 & 10.17 & 30.02 & 1651 \\
\hline $\begin{array}{l}\text { Bacteria Actinobacteria Actinobacteria } \\
\text { Actinomycetales Gordoniaceae Gordonia }\end{array}$ & 5.94 & 4.68 & 6.67 & 6.79 & 36.81 & 10562 \\
\hline $\begin{array}{l}\text { Bacteria Proteobacteria Alphaproteobacteria } \\
\text { Rhizobiales Bradyrhizobiaceae }\end{array}$ & 3.78 & 3.22 & 4.68 & 4.68 & 41.49 & 10599 \\
\hline $\begin{array}{l}\text { Bacteria Bacteroidetes Cytophagia } \\
\text { Cytophagales Cytophagaceae Sporocytophaga }\end{array}$ & 3.8 & 2.97 & 3.87 & 4.3 & 45.79 & 8299 \\
\hline $\begin{array}{l}\text { Bacteria Planctomycetes Planctomycetia } \\
\text { Pirellulales Pirellulaceae A17 }\end{array}$ & 3.3 & 2.79 & 4.85 & 4.04 & 49.84 & 8792 \\
\hline $\begin{array}{l}\text { Bacteria Planctomycetes Planctomycetia } \\
\text { Gemmatales Isosphaeraceae }\end{array}$ & 3.27 & 2.46 & 2.81 & 3.57 & 53.41 & 4563 \\
\hline $\begin{array}{l}\text { Bacteria Proteobacteria Alphaproteobacteria } \\
\text { Rhizobiales }\end{array}$ & 2.54 & 2.11 & 5.9 & 3.06 & 56.47 & 14302 \\
\hline
\end{tabular}




\section{Type: Gut, Day: 7, System: RAS}

Average similarity: 55.06

Taxonomic ID

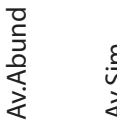

Bacteria Proteobacteria Alphaproteobacteria

$\begin{array}{llllll}18.92 & 11.59 & 1.29 & 21.06 & 21.06 & 10599\end{array}$

Rhizobiales Bradyrhizobiaceae

Bacteria Actinobacteria Actinobacteria

$\begin{array}{llllll}15.17 & 9.28 & 1.37 & 16.86 & 37.91 & 1005\end{array}$

Actinomycetales Nocardiaceae Rhodococcus

Bacteria Actinobacteria Actinobacteria Actinomycetales

$\begin{array}{llllll}11.43 & 9.02 & 3.02 & 16.38 & 54.3 & 4790\end{array}$

Mycobacteriaceae Mycobacterium llatzerense

Bacteria Actinobacteria Actinobacteria

$\begin{array}{llllll}6.8 & 4.66 & 2 & 8.47 & 62.77 & 12555\end{array}$

Actinomycetales Microbacteriaceae Agrococcus

Type: Gut, Day: 7, System: AS vs. RAS

Average dissimilarity $=78.38$

\begin{tabular}{|c|c|c|c|c|c|c|c|}
\hline & $\begin{array}{l}\frac{n}{4} \\
\frac{0}{3} \\
0 \\
0\end{array}$ & 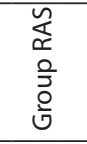 & & & & & \\
\hline Taxonomic ID & 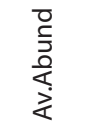 & 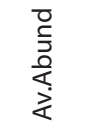 & $\frac{\cong}{\stackrel{n}{0}}$ & $\begin{array}{l}\stackrel{0}{\curvearrowleft} \\
\stackrel{n}{\Delta}\end{array}$ & 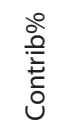 & 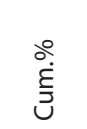 & $\stackrel{?}{\circ}$ \\
\hline $\begin{array}{l}\text { Bacteria Proteobacteria Alphaproteobacteria Rhizobiales } \\
\text { Bradyrhizobiaceae }\end{array}$ & 3.78 & 18.92 & 7.58 & 1.23 & 9.67 & 9.67 & 10599 \\
\hline $\begin{array}{l}\text { Bacteria Actinobacteria Actinobacteria Actinomycetales } \\
\text { Nocardiaceae Rhodococcus }\end{array}$ & 0.44 & 15.17 & 7.36 & 1.52 & 9.39 & 19.07 & 1005 \\
\hline $\begin{array}{l}\text { Bacteria Actinobacteria Actinobacteria Actinomycetales } \\
\text { Mycobacteriaceae Mycobacterium llatzerense }\end{array}$ & 19.37 & 11.43 & 5.14 & 1.35 & 6.56 & 25.63 & 4790 \\
\hline $\begin{array}{l}\text { Bacteria Proteobacteria Alphaproteobacteria } \\
\text { Rhodobacterales Rhodobacteraceae Rhodobacter }\end{array}$ & 9.35 & 0 & 4.68 & 2.51 & 5.96 & 31.59 & 1651 \\
\hline $\begin{array}{l}\text { Bacteria Actinobacteria Actinobacteria Actinomycetales } \\
\text { Microbacteriaceae Agrococcus }\end{array}$ & 0.01 & 6.8 & 3.4 & 1.9 & 4.33 & 35.93 & 12555 \\
\hline $\begin{array}{l}\text { Bacteria Actinobacteria Actinobacteria Actinomycetales } \\
\text { Gordoniaceae Gordonia }\end{array}$ & 5.94 & 2.36 & 3.27 & 1.59 & 4.17 & 40.1 & 10562 \\
\hline $\begin{array}{l}\text { Bacteria Actinobacteria Thermoleophilia } \\
\text { Solirubrobacterales }\end{array}$ & 0 & 5.79 & 2.89 & 2.72 & 3.69 & 43.79 & 3934 \\
\hline
\end{tabular}


Type: Water, Day: 7, System: AS

Average similarity: 63.75

Taxonomic ID

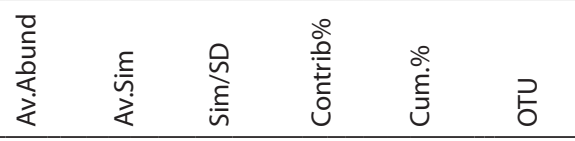

Bacteria Proteobacteria Alphaproteobacteria

Sphingomonadales

$\begin{array}{cccccc}18.3 & 14.75 & \text { \#\#\#\#\#\#\# } & 23.13 & 23.13 & 9828 \\ & & & & & \\ 7.03 & 5.81 & \text { \#\#\#\#\#\#\# } & 9.12 & 32.25 & 4552 \\ & & & & & \\ 4.29 & 4.1 & \text { \#\#\#\#\#\#\# } & 6.43 & 38.67 & 8066 \\ & & & & & \\ 5 & 3.81 & \text { \#\#\#\#\#\#\# } & 5.98 & 44.66 & 2756 \\ & & & & & \\ 4.1 & 2.96 & \text { \#\#\#\#\#\#\# } & 4.64 & 49.3 & 11945\end{array}$

Bacteria Proteobacteria Betaproteobacteria Burkholderiales Comamonadaceae Limnohabitans

stem: RAS

\section{Type: Water, Day: 7, System: RAS}

Average similarity: 58.96

\begin{tabular}{|c|c|c|c|c|c|c|}
\hline Taxonomic ID & 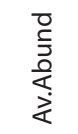 & 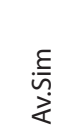 & $\underset{\text { है }}{\stackrel{0}{n}}$ & 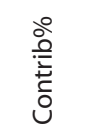 & 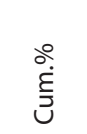 & $\stackrel{?}{\circ}$ \\
\hline $\begin{array}{l}\text { Bacteria Proteobacteria Betaproteobacteria Burkholderiales } \\
\text { Oxalobacteraceae Polynucleobacter }\end{array}$ & 31.16 & 24.13 & 3.1 & 40.92 & 40.92 & 7333 \\
\hline $\begin{array}{l}\text { Bacteria Proteobacteria Alphaproteobacteria } \\
\text { Sphingomonadales }\end{array}$ & 15.66 & 9.97 & 1.67 & 16.91 & 57.83 & 9828 \\
\hline
\end{tabular}

Type: Water, Day: 7, System: AS vs. RAS

Average dissimilarity $=76.04$

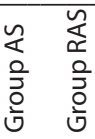

Taxonomic ID

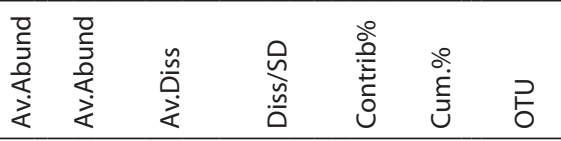

Bacteria Proteobacteria Betaproteobacteria Burkholderiales

Oxalobacteraceae Polynucleobacter

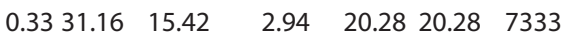

Bacteria Proteobacteria Alphaproteobacteria

Sphingomonadales

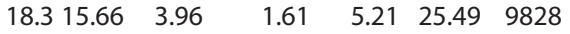

Bacteria Proteobacteria Alphaproteobacteria

Sphingomonadales

$\begin{array}{lllllll}7.03 & 0.04 & 3.49 & 5.37 & 4.6 & 30.08 & 4552\end{array}$

Bacteria Proteobacteria Alphaproteobacteria

Sphingomonadales

$\begin{array}{lllllll}1 & 5.25 & 2.49 & 1.09 & 3.28 & 33.36 & 10813\end{array}$

Bacteria Proteobacteria Alphaproteobacteria Rhodobacterales Rhodobacteraceae Rhodobacter

$\begin{array}{lllllll}5 & 0.11 & 2.44 & 3.86 & 3.21 & 36.57 & 2756\end{array}$ 
Bacteria Bacteroidetes Saprospirae Saprospirales

Chitinophagaceae Sediminibacterium

Bacteria Proteobacteria Alphaproteobacteria

Sphingomonadales

Bacteria Nitrospirae Nitrospira Nitrospirales Nitrospiraceae Nitrospira

Bacteria Actinobacteria Actinobacteria Actinomycetales Mycobacteriaceae Mycobacterium Ilatzerense

Bacteria Actinobacteria Actinobacteria Actinomycetales Mycobacteriaceae Mycobacterium Ilatzerense

Bacteria Proteobacteria Betaproteobacteria Burkholderiales Comamonadaceae

Bacteria Armatimonadetes Armatimonadia Armatimonadales

Armatimonadaceae Armatimonas

Bacteria Proteobacteria Alphaproteobacteria

Sphingomonadales Sphingomonadaceae Novosphingobium

\begin{tabular}{llllllll}
4.29 & 0.01 & 2.14 & 20.93 & 2.81 & 39.38 & 8066 \\
4.22 & 0 & 2.11 & 2.55 & 2.77 & 42.15 & 4582 \\
4.1 & 0.18 & 1.96 & 3.19 & 2.58 & 44.73 & 11945 \\
1.23 & 3.39 & 1.4 & 1.31 & 1.85 & 46.58 & 4790 \\
0.01 & 2.72 & 1.36 & 0.94 & 1.79 & 48.36 & 9738 \\
2.4 & 0.29 & 1.17 & 1.03 & 1.54 & 49.9 & 12945 \\
& & & & & & \\
\hline 2.09 & 0 & 1.05 & 1.02 & 1.38 & 51.28 & 7261 \\
0.78 & 2.6 & 0.92 & 0.99 & 1.21 & 52.49 & 6299 \\
\hline
\end{tabular}

\section{Type: Gut, Day: 42, System: AS}

Average similarity: 59.85

\begin{tabular}{|c|c|c|c|c|c|c|}
\hline Taxonomic ID & 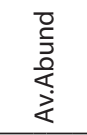 & 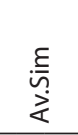 & $\underset{\text { है }}{\stackrel{0}{n}}$ & 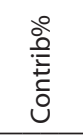 & ฏ̊ & $\stackrel{?}{\circ}$ \\
\hline $\begin{array}{l}\text { Bacteria Planctomycetes Planctomycetia Gemmatales } \\
\text { Isosphaeraceae }\end{array}$ & 40.76 & 27.08 & 1.56 & 45.25 & 45.25 & 4563 \\
\hline $\begin{array}{l}\text { Bacteria Actinobacteria Actinobacteria Actinomycetales } \\
\text { Micrococcaceae Arthrobacter }\end{array}$ & 19.29 & 14.57 & 3.77 & 24.34 & 69.59 & 12537 \\
\hline $\begin{array}{l}\text { Bacteria Actinobacteria Actinobacteria Actinomycetales } \\
\text { Mycobacteriaceae Mycobacterium llatzerense }\end{array}$ & 16.17 & 8.74 & 0.97 & 14.6 & 84.19 & 4790 \\
\hline
\end{tabular}

Type: Gut, Day: 42, System: RAS

Average similarity: 56.35

\begin{tabular}{|c|c|c|c|c|c|c|}
\hline Taxonomic ID & 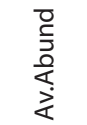 & 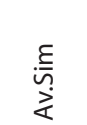 & $\stackrel{\vartheta}{\stackrel{0}{n}}$ & 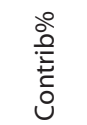 & $\stackrel{\circ}{\dot{\xi}}$ & $\stackrel{?}{\circ}$ \\
\hline $\begin{array}{l}\text { Bacteria Firmicutes Clostridia Clostridiales } \\
\text { Peptostreptococcaceae }\end{array}$ & 18.13 & 13.02 & 2.8 & 23.11 & 23.11 & 7966 \\
\hline $\begin{array}{l}\text { Bacteria Actinobacteria Actinobacteria Actinomycetales } \\
\text { Mycobacteriaceae Mycobacterium llatzerense }\end{array}$ & 12.06 & 9.44 & 2.99 & 16.75 & 39.86 & 4790 \\
\hline Bacteria Firmicutes Clostridia Clostridiales [Mogibacteriaceae] & 8.4 & 6.16 & 2.21 & 10.93 & 50.79 & 7955 \\
\hline $\begin{array}{l}\text { Bacteria Actinobacteria Actinobacteria Actinomycetales } \\
\text { Nocardiaceae Rhodococcus }\end{array}$ & 9.21 & 5.74 & 1.73 & 10.18 & 60.96 & 1005 \\
\hline $\begin{array}{l}\text { Bacteria Fusobacteria Fusobacteria Fusobacteriales } \\
\text { Fusobacteriaceae Cetobacterium somerae }\end{array}$ & 11.73 & 4.31 & 0.75 & 7.64 & 68.61 & 2787 \\
\hline
\end{tabular}




\section{Type: Gut, Day: 42, System: AS vs. RAS}

Average dissimilarity $=89.22$

$\begin{array}{ll}n & \text { n } \\ \frac{1}{2} & 0 \\ \frac{0}{2} & \frac{0}{3} \\ 0 & 0 \\ 0 & \frac{0}{0}\end{array}$

Taxonomic ID

\begin{tabular}{|c|c|c|c|c|c|c|}
\hline 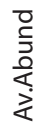 & 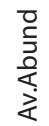 & 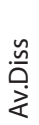 & $\begin{array}{l}\stackrel{0}{\tilde{n}} \\
\stackrel{n}{\Delta}\end{array}$ & 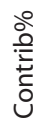 & $\stackrel{\circ}{\stackrel{\varepsilon}{J}}$ & 0 \\
\hline
\end{tabular}

Bacteria Planctomycetes Planctomycetia Gemmatales Isosphaeraceae

\begin{tabular}{|c|c|c|c|c|c|c|}
\hline 40.76 & 0 & 20.38 & 1.91 & 22.84 & 22.84 & 4563 \\
\hline 19.29 & 0.01 & 9.64 & 2.57 & 10.8 & 33.64 & 12537 \\
\hline 0.04 & 18.13 & 9.04 & 2.01 & 10.14 & 43.78 & 7966 \\
\hline 0 & 11.73 & 5.86 & 0.85 & 6.57 & 50.35 & 2787 \\
\hline 16.17 & 12.06 & 5.78 & 1.87 & 6.48 & 56.83 & 4790 \\
\hline 0 & 9.21 & 4.6 & 1.56 & 5.16 & 61.99 & 1005 \\
\hline 0.01 & 8.4 & 4.2 & 2.3 & 4.7 & 66.69 & 7955 \\
\hline 6.02 & 0 & 3.01 & 0.78 & 3.37 & 70.07 & 2401 \\
\hline 1.77 & 2.63 & 1.53 & 1.07 & 1.72 & 71.79 & 12852 \\
\hline 0.01 & 3.04 & 1.51 & 0.99 & 1.7 & 73.48 & 12238 \\
\hline 0 & 2.73 & 1.37 & 1.15 & 1.53 & 75.01 & 3934 \\
\hline
\end{tabular}

Bacteria Actinobacteria Actinobacteria Actinomycetales Micrococcaceae Arthrobacter

Bacteria Firmicutes Clostridia Clostridiales

Peptostreptococcaceae

Bacteria Fusobacteria Fusobacteria Fusobacteriales Fusobacteriaceae Cetobacterium somerae

Bacteria Actinobacteria Actinobacteria Actinomycetales Mycobacteriaceae Mycobacterium Ilatzerense

Bacteria Actinobacteria Actinobacteria Actinomycetales Nocardiaceae Rhodococcus

Bacteria Firmicutes Clostridia Clostridiales

Mogibacteriaceae

Bacteria Proteobacteria Gammaproteobacteria

Legionellales Legionellaceae Tatlockia

Bacteria Proteobacteria Gammaproteobacteria

Enterobacteriales Enterobacteriaceae Plesiomonas

shigelloides

Bacteria Actinobacteria Actinobacteria Actinomycetales Mycobacteriaceae Mycobacterium celatum

Bacteria Actinobacteria Thermoleophilia

Solirubrobacterales

$\frac{n}{1}$
$\frac{n}{2}$
$\frac{0}{2}$

\section{Type: Water, Day: 42, System: AS}

Average similarity: 25.82

Taxonomic ID

\begin{tabular}{|c|c|c|c|c|c|}
\hline $\begin{array}{l}\text { ర్ } \\
\text { క }\end{array}$ & 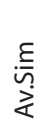 & 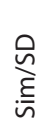 & 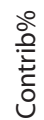 & 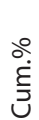 & ? \\
\hline
\end{tabular}

Bacteria Bacteroidetes Saprospirae Saprospirales

Chitinophagaceae Sediminibacterium

$20.31 \quad 11.43$ \#\#\#\#\#\#\# $44.28 \quad 44.28 \quad 8066$

Bacteria Proteobacteria Betaproteobacteria

Burkholderiales Comamonadaceae

$5.25 \quad 2.9 \quad$ \#\#\#\#\#\#\# $11.24 \quad 55.52 \quad 14254$

Bacteria Proteobacteria Alphaproteobacteria

Rhodospirillales Rhodospirillaceae

Bacteria Bacteroidetes Sphingobacteria Sphingobacteriales 


\section{Type: Water, Day: 42, System: RAS}

Average similarity: 60.79

Taxonomic ID

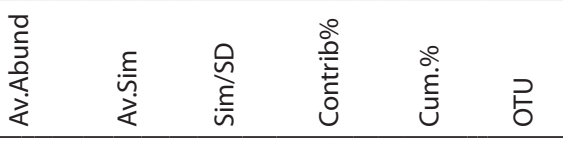

Bacteria Proteobacteria Betaproteobacteria

Rhodocyclales Rhodocyclaceae

$\begin{array}{llllll}35.64 & 30.69 & 3.97 & 50.48 & 50.48 & 7090\end{array}$

Bacteria Proteobacteria Betaproteobacteria

Burkholderiales Comamonadaceae Limnohabitans

$\begin{array}{llllll}10.73 & 6.8 & 4.31 & 11.18 & 61.66 & 14286\end{array}$

Bacteria Proteobacteria Alphaproteobacteria

Rhodobacterales Rhodobacteraceae Rhodobacter

$\begin{array}{llllll}12.56 & 3.32 & 0.41 & 5.47 & 67.13 & 4377\end{array}$

Type: Water, Day: 42, System: AS vs. RAS

Average dissimilarity $=98.24$

\begin{tabular}{|c|c|c|c|c|c|c|c|}
\hline & $\begin{array}{l}\text { 足 } \\
\text { 음 } \\
\text { 인 }\end{array}$ & 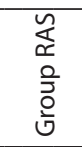 & & & & & \\
\hline Taxonomic ID & 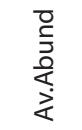 & 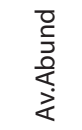 & $\frac{n}{\stackrel{n}{0}}$ & $\begin{array}{l}0 \\
\stackrel{n}{0} \\
\frac{n}{0}\end{array}$ & 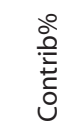 & 良 & $\stackrel{?}{\circ}$ \\
\hline $\begin{array}{l}\text { Bacteria Proteobacteria Betaproteobacteria } \\
\text { Rhodocyclales Rhodocyclaceae }\end{array}$ & 0.01 & 35.64 & 17.82 & 4.63 & 18.14 & 18.14 & 7090 \\
\hline $\begin{array}{l}\text { Bacteria Bacteroidetes Saprospirae Saprospirales } \\
\text { Chitinophagaceae Sediminibacterium }\end{array}$ & 20.31 & 0.07 & 10.12 & 2.13 & 10.3 & 28.43 & 8066 \\
\hline $\begin{array}{l}\text { Bacteria Armatimonadetes Fimbriimonadia } \\
\text { Fimbriimonadales Fimbriimonadaceae Fimbriimonas }\end{array}$ & 14.54 & 0 & 7.27 & 0.94 & 7.4 & 35.84 & 5688 \\
\hline $\begin{array}{l}\text { Bacteria Proteobacteria Alphaproteobacteria } \\
\text { Rhodobacterales Rhodobacteraceae Rhodobacter }\end{array}$ & 0 & 12.56 & 6.28 & 0.9 & 6.39 & 42.23 & 4377 \\
\hline $\begin{array}{l}\text { Bacteria Proteobacteria Betaproteobacteria } \\
\text { Burkholderiales Comamonadaceae Limnohabitans }\end{array}$ & 0.01 & 10.73 & 5.36 & 1.71 & 5.46 & 47.68 & 14286 \\
\hline $\begin{array}{l}\text { Bacteria Armatimonadetes Armatimonadia } \\
\text { Armatimonadales Armatimonadaceae Armatimonas }\end{array}$ & 8.11 & 0 & 4.05 & 0.94 & 4.13 & 51.81 & 7261 \\
\hline $\begin{array}{l}\text { Bacteria Bacteroidetes Sphingobacteria } \\
\text { Sphingobacteriales }\end{array}$ & 7.02 & 0 & 3.51 & 1.08 & 3.57 & 55.39 & 4686 \\
\hline $\begin{array}{l}\text { Bacteria Proteobacteria Betaproteobacteria } \\
\text { Burkholderiales Comamonadaceae }\end{array}$ & 5.25 & 0 & 2.63 & 2.09 & 2.67 & 58.06 & 14254 \\
\hline $\begin{array}{l}\text { Bacteria Fusobacteria Fusobacteria Fusobacteriales } \\
\text { Fusobacteriaceae Cetobacterium somerae }\end{array}$ & 0 & 5.02 & 2.51 & 1.58 & 2.55 & 60.61 & 2787 \\
\hline $\begin{array}{l}\text { Bacteria Actinobacteria Actinobacteria Actinomycetales } \\
\text { ACK-M1 }\end{array}$ & 0 & 4.96 & 2.48 & 0.9 & 2.52 & 63.14 & 5270 \\
\hline
\end{tabular}




\section{Type: Feed}

Average similarity: 59.36

Taxonomic ID

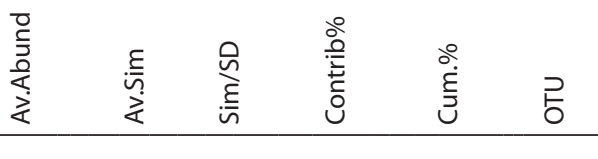

Bacteria Proteobacteria Gammaproteobacteria

Vibrionales Vibrionaceae Photobacterium

10.9

9.94 \#\#\#\#\#\#\# 16.75

$16.75 \quad 14212$

Bacteria Fusobacteria Fusobacteria Fusobacteriales

Fusobacteriaceae Psychrilyobacter

$\begin{array}{lllll}7.11 & 6.78 & \# \# \# \# \# \# \quad 11.42 \quad 28.16 \quad 2650\end{array}$

Bacteria Firmicutes Bacilli Lactobacillales

Aerococcaceae Facklamia

$3.94 \quad 3.83$ \#\#\#\#\#\#\# $6.46 \quad 34.62 \quad 3532$

Bacteria Firmicutes Bacilli Bacillales Bacillaceae

Anoxybacillus kestanbolensis

$\begin{array}{lllll}4.12 & 3.78 & \text { \#\#\#\#\#\#\# } 6.37 & 40.99 & 1521\end{array}$

Bacteria Firmicutes Bacilli Bacillales Planococcaceae

Sporosarcina

3.2 \#\#\#\#\#\# 5.39

46.38

3747

Bacteria Actinobacteria Actinobacteria

Actinomycetales Corynebacteriaceae

12.34

2.94 \#\#\#\#\#\#\# 4.95

51.32

5872

Corynebacterium

Table S3.2. Average alpha-diversity indices of gut, water and feed bacterial communities. Number of obtained sequences was standardized among samples to the minimum number of reads obtained in one of the samples $(n=1719)$. S: Richness (observed OTUs); J': Pielou's evenness $\left(J^{\prime}=H^{\prime} / \log (S)\right) ; H^{\prime}:$ Shannon index $\left(H^{\prime}=-S U M\left(P_{i}^{*} \ln \left(P_{i}\right)\right)\right)$, based on the OTU level. AS \& RAS: Active suspension and recirculating aquaculture systems; 07 \& 42 : Sampling days 7 and 42 respectively.

\begin{tabular}{lccc}
\hline Sampling Group & $\mathrm{S}$ & $\mathrm{J}$ & $\mathrm{H}^{\prime}(\mathrm{In})$ \\
\hline Gut_07_AS & 732 & 0.626 & 4.132 \\
Gut_07_RAS & 1003 & 0.532 & 3.674 \\
Gut_42_AS & 279 & 0.395 & 2.225 \\
Gut_42_RAS & 879 & 0.519 & 3.521 \\
\hline Water_07_AS & 683 & 0.690 & 4.504 \\
Water_07_RAS & 815 & 0.540 & 3.618 \\
Water_42_AS & 394 & 0.601 & 3.594 \\
Water_42_RAS & 552 & 0.494 & 3.118 \\
\hline Feed & 406 & 0.666 & 3.997 \\
\hline
\end{tabular}


Table S3.3. RELATE analysis testing for matched resemblance matrices based on Spearman's rank correlation coefficient. Water and gut microbiota data sets were square root transformed and resemblance matrices were based on Bray Curtis similarity among samples. Rho statistic indicates the degree of "relatedness" between the two matrices with highly related matrices having Rho statistic closer to one. BCs: Bacterial communities.

\begin{tabular}{lc}
\hline Resemblance matrices & Water BCs \\
& Vs. \\
Gut BCs \\
\hline Rank correlation method & Spearman's p \\
Sample statistic (Rho) & 0.807 \\
Significance level of sample statistic & 0.0001 \\
Number of permutations & 9999 \\
Number of permuted statistics greater than or equal to Rho & 0 \\
\hline
\end{tabular}

Table S3.4. List of the most predominant gut, water and feed OTUs on day 7 and 42 as presented on the heatmap Table 3. Column "Processes" and "Environment" provide information about the major functions and processes as well as the environments in which the most predominant OTUs were encountered as predicted based on a literature review (column "References").

\begin{tabular}{|c|c|c|c|c|}
\hline Taxonomy & $\begin{array}{l}\text { OTU } \\
\text { number }\end{array}$ & Processes & Environment & References \\
\hline $\begin{array}{l}\text { Actinobacteria } \\
\text { Actinomycetales } \\
\text { Mycobacteriaceae } \\
\text { Mycobacterium } \\
\text { Ilatzerense }\end{array}$ & 4790 & $\begin{array}{l}\text { Facultative autotropic, } \\
\text { aerobic, hydrogen } \\
\text { oxidation }\end{array}$ & $\begin{array}{l}\text { Haemodialysis water; tap } \\
\text { water production plant } \\
\text { and distribution system }\end{array}$ & $\begin{array}{l}\text { (Dubrou et al., 2013; } \\
\text { Gomila et al., 2008) }\end{array}$ \\
\hline $\begin{array}{l}\text { Proteobacteria } \\
\text { Alphaproteobacteria } \\
\text { Rhodobacterales } \\
\text { Rhodobacteraceae } \\
\text { Rhodobacter }\end{array}$ & 1651 & $\begin{array}{l}\text { Non-sulphur purple } \\
\text { bacteria, photosynthesis, } \\
\text { lithotrophs, aerobic and } \\
\text { anaerobic respiration, } \mathrm{N}_{2} \\
\text { fixation }\end{array}$ & $\begin{array}{l}\text { Hypersaline, saline and } \\
\text { freshwater }\end{array}$ & $\begin{array}{l}\text { (Bräuer et al., 2011; } \\
\text { Glöckner et al., 1999; } \\
\text { Hiraishi et al., 1995; } \\
\text { Kawasaki et al., 1993; } \\
\text { Stapleton et al., 2000; } \\
\text { Yang et al., 2012) }\end{array}$ \\
\hline $\begin{array}{l}\text { Actinobacteria } \\
\text { Actinobacteria } \\
\text { Actinomycetales } \\
\text { Gordoniaceae Gordonia }\end{array}$ & 10562 & $\begin{array}{l}\text { High GC-content, Gram+ } \\
\text { bacteria, degrade many } \\
\text { xenobiotics, potentially } \\
\text { pathogenic. }\end{array}$ & $\begin{array}{l}\text { Soil and mangrove } \\
\text { rhizospheres, waste } \\
\text { water treatment plants, } \\
\text { diseased humans, } \\
\text { hydrocarbon polluted soil, } \\
\text { oil wells }\end{array}$ & $\begin{array}{l}\text { (Arenskötter et al., 2004) } \\
\text {, }\end{array}$ \\
\hline $\begin{array}{l}\text { Proteobacteria } \\
\text { Alphaproteobacteria } \\
\text { Rhizobiales } \\
\text { Bradyrhizobiaceae }\end{array}$ & 10599 & $\begin{array}{l}\text { Contains } 10 \text { genera, } \\
\text { rhizobium associated } \\
\text { and animal associated } \\
\text { (e.g. cat scratch disease). } \\
\text { Many free living in } \\
\text { marine or fresh water, } \\
\text { some with } \mathrm{N}_{2} \text {-fixation. }\end{array}$ & $\begin{array}{l}\text { Sewage treatment } \\
\text { plants, municipal waste } \\
\text { waters, drinking water } \\
\text { distribution. In gut of } \\
\text { sea bass and stomach of } \\
\text { yellow catfish. }\end{array}$ & $\begin{array}{l}\text { (Liu et al., 2012b; Wang } \\
\text { et al., 2013; Yang et al., } \\
\text { 2012; Ye and Zhang, } \\
\text { 2013; Zhang et al., 2012) }\end{array}$ \\
\hline
\end{tabular}




\begin{tabular}{|c|c|c|c|c|}
\hline Taxonomy & $\begin{array}{l}\text { OTU } \\
\text { number }\end{array}$ & Processes & Environment & References \\
\hline $\begin{array}{l}\text { Actinobacteria } \\
\text { Actinobacteria } \\
\text { Actinomycetales } \\
\text { Nocardiaceae } \\
\text { Rhodococcus }\end{array}$ & 1005 & $\begin{array}{l}\text { Chemoheterotrophic, } \\
\text { some members are } \\
\text { facultative chemo- } \\
\text { lithoauthrophs. } \\
\text { Many processes. } \\
\text { Some species used as } \\
\text { immunostimulants, } \\
\text { others for production } \\
\text { of digestive enzymes. }\end{array}$ & $\begin{array}{l}\text { Wide distribution, } \\
\text { including soil, water } \\
\text { and in cells. Reported } \\
\text { in sole, red rock fish, } \\
\text { Norwegian mackerel, } \\
\text { USA smelt, rainbow } \\
\text { trout and shrimp gut. }\end{array}$ & $\begin{array}{l}\text { (Boutin et al., 2013a; } \\
\text { Khan et al., 2011; Kim } \\
\text { et al., 2007; Sanchez et } \\
\text { al., 2012; Sharifuzzaman } \\
\text { et al., 2014; Spanggaard } \\
\text { et al., 2000; Tinh et al., } \\
\text { 2007) }\end{array}$ \\
\hline $\begin{array}{l}\text { Proteobacteria } \\
\text { Alphaproteobacteria } \\
\text { Sphingomonadales }\end{array}$ & 9828 & $\begin{array}{l}\text { No info about functional } \\
\text { role. Sphingolipids in } \\
\text { outer membrane of cell } \\
\text { wall. }\end{array}$ & $\begin{array}{l}\text { Drinking water systems, } \\
\text { groundwater treatment } \\
\text { biofilms. }\end{array}$ & $\begin{array}{l}\text { (Holinger et al., 2014; } \\
\text { Liu et al., 2012a, 2012b; } \\
\text { Ultee et al., 2004; Vílchez } \\
\text { et al., 2011) }\end{array}$ \\
\hline $\begin{array}{l}\text { Proteobacteria } \\
\text { Betaproteobacteria } \\
\text { Burkholderiales } \\
\text { Comamonadaceae } \\
\text { Limnohabitans }\end{array}$ & 4552 & $\begin{array}{l}\text { Aerobic, facultative } \\
\text { anaerobic, chemo- } \\
\text { organotrophic. Affinity } \\
\text { for substrates } \\
\text { with organic acids, } \\
\text { monosaccharides and } \\
\text { amino acids. }\end{array}$ & $\begin{array}{l}\text { Bacteria found in water } \\
\text { column of freshwaters. }\end{array}$ & $\begin{array}{l}\text { (Hahn et al., 2010; } \\
\text { Jezbera et al., 2013; } \\
\text { Kasalický et al., 2010; } \\
\text { Šimek et al., 2013; Zeng } \\
\text { et al., 2012; Kasalický et } \\
\text { al., 2013) }\end{array}$ \\
\hline $\begin{array}{l}\text { Bacteroidetes } \\
\text { Saprospirae } \\
\text { Saprospirales } \\
\text { Chitinophagaceae } \\
\text { Sediminibacterium }\end{array}$ & 8066 & $\begin{array}{l}\text { Versatile in types of } \\
\text { substrates that are } \\
\text { degraded. Aerobic or } \\
\text { facultative anaerobic. }\end{array}$ & $\begin{array}{l}\text { Lakes, freshwater } \\
\text { reservoirs, activated } \\
\text { sludge reactors, stream } \\
\text { biofilms, soil }\end{array}$ & $\begin{array}{l}\text { (Besemer et al., 2012; } \\
\text { Khemkhao et al., 2011; } \\
\text { Kim et al., 2013; Qu and } \\
\text { Yuan, 2008; Singleton et } \\
\text { al., 2011; Torrentó et al., } \\
\text { 2011) }\end{array}$ \\
\hline
\end{tabular}




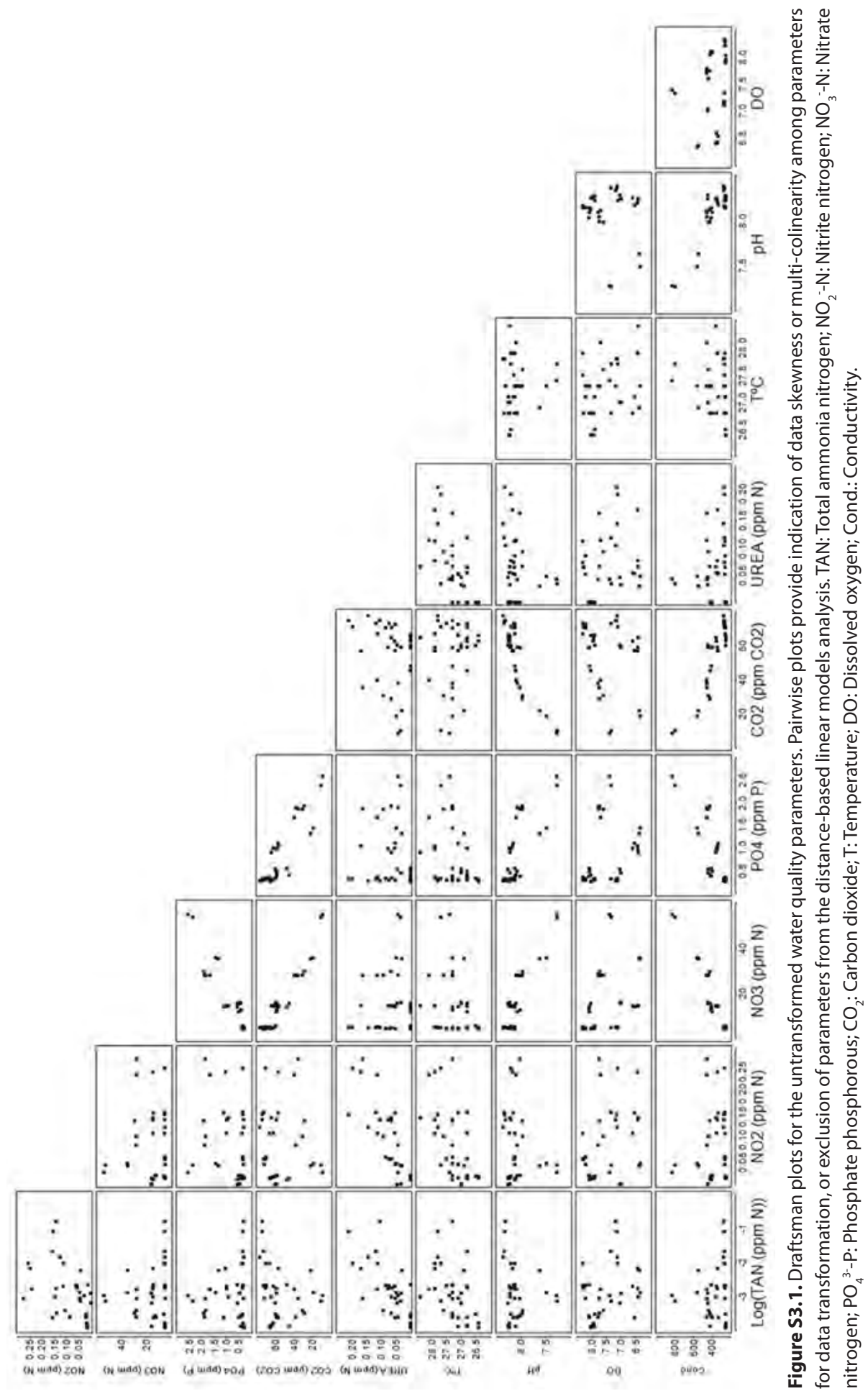



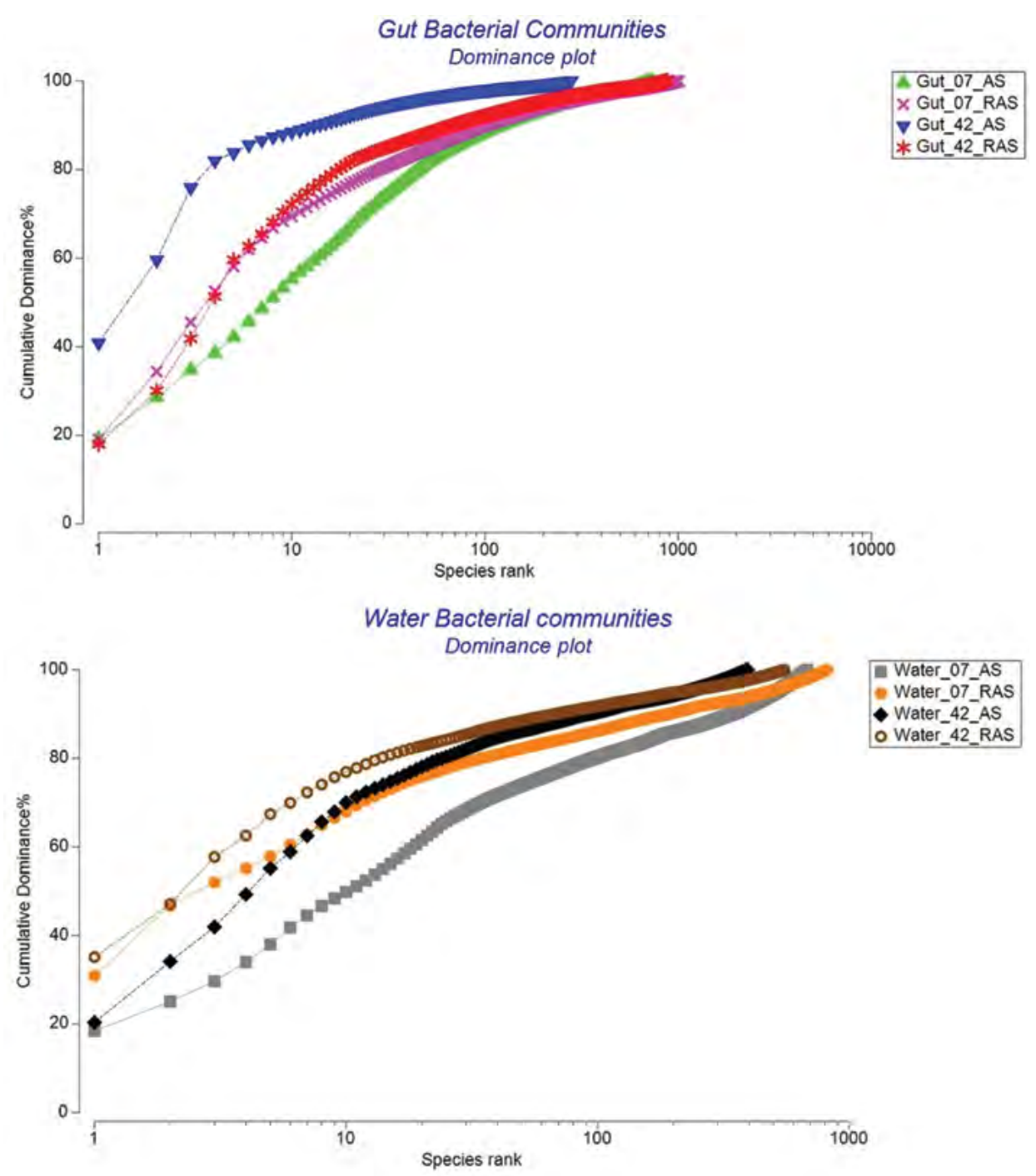

Figure S3.2. Dominance plots of gut and water OTUs from the two experimental days. Plots are based on average relative Bray Curtis OTU abundance from each system. The $x$-axis shows the log scale of the OTUs ranks in increasing order and the $y$-axis consists of the cumulative relative abundance of each OTU in each sample group. 07 and 42: sampling day 7 \& 42; AS \& RAS: Active suspension and recirculating aquaculture systems respectively. 


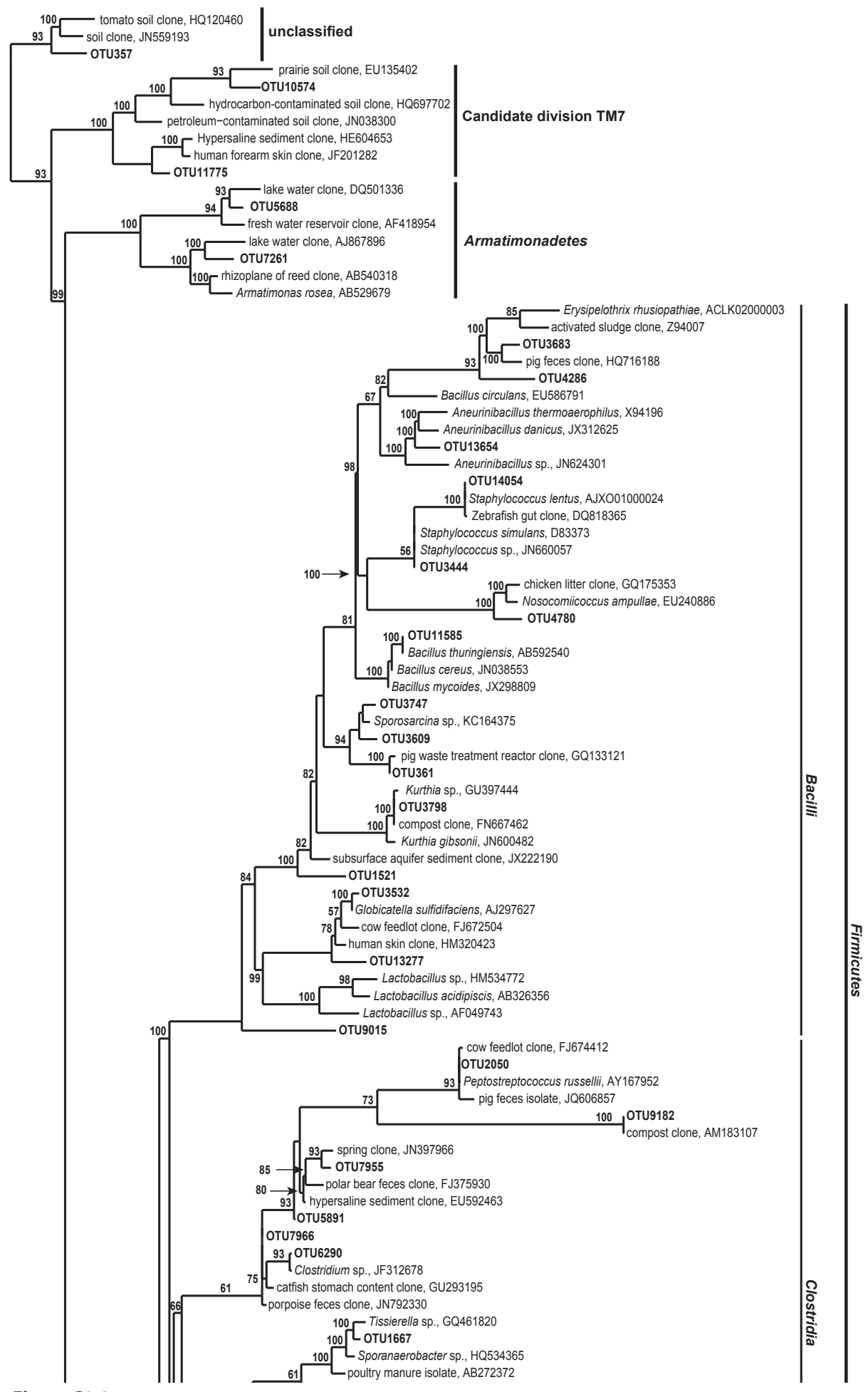

Figure S3.3. 


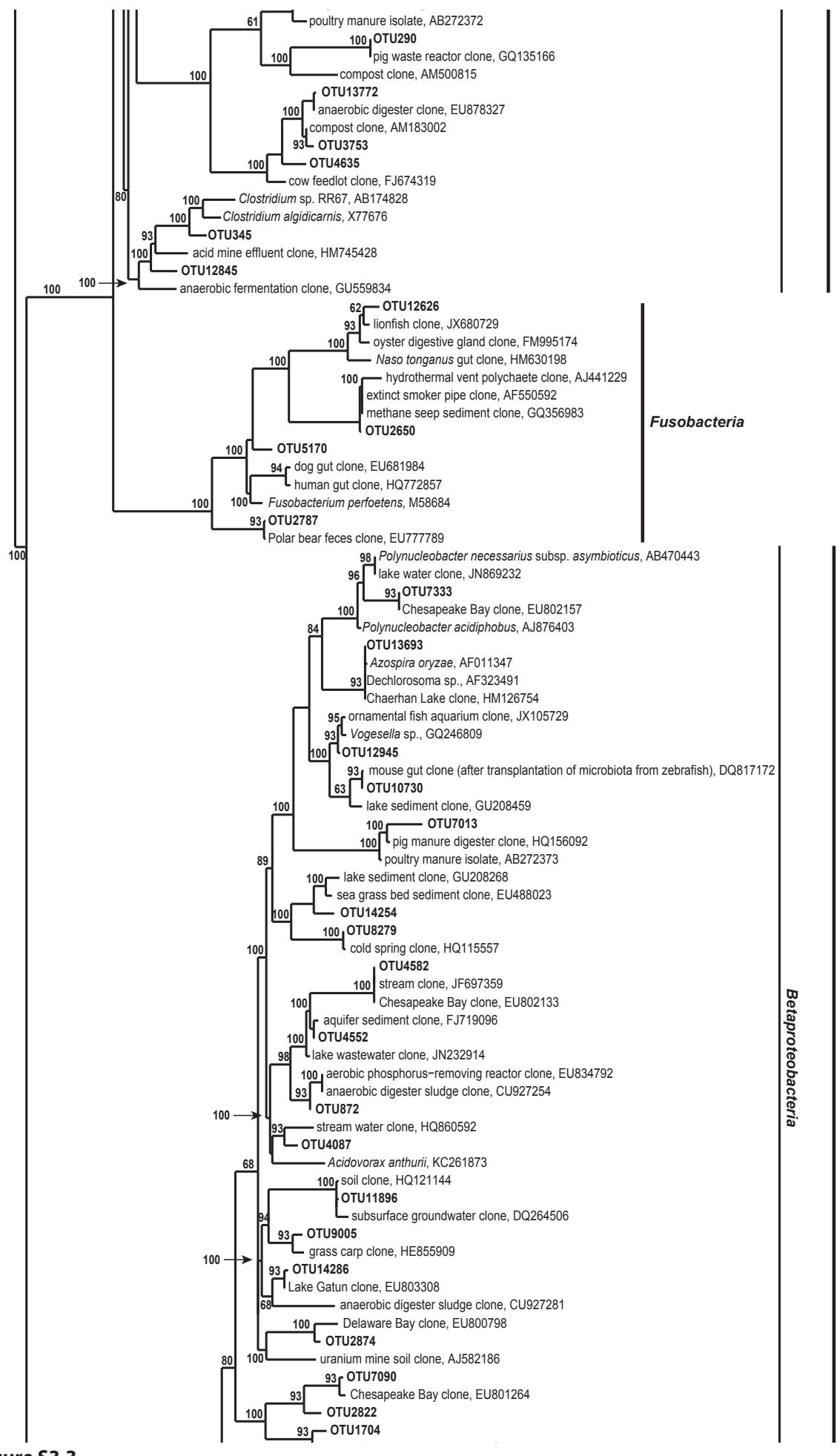

Figure S3.3. 


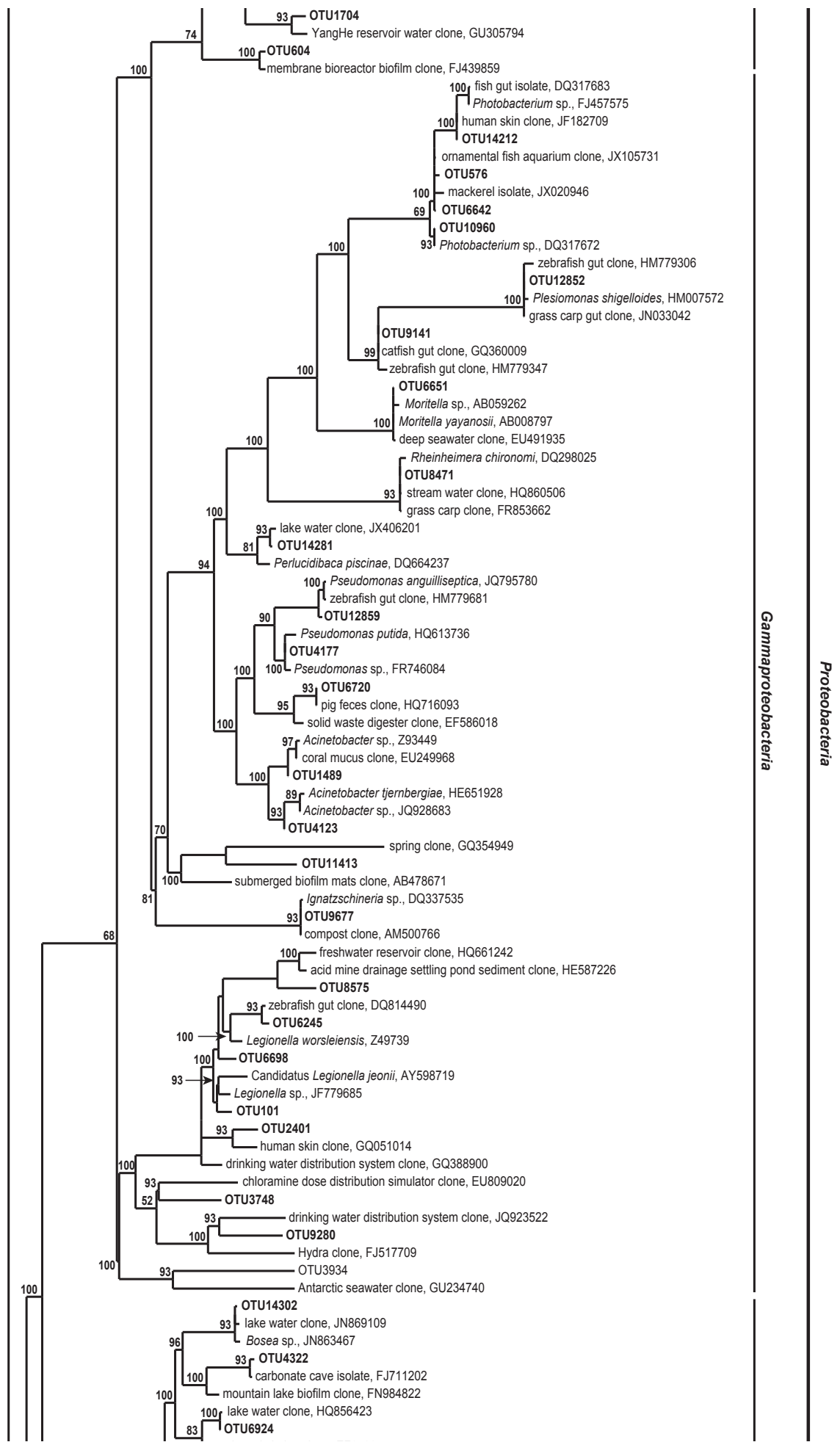

Figure S3.3. 

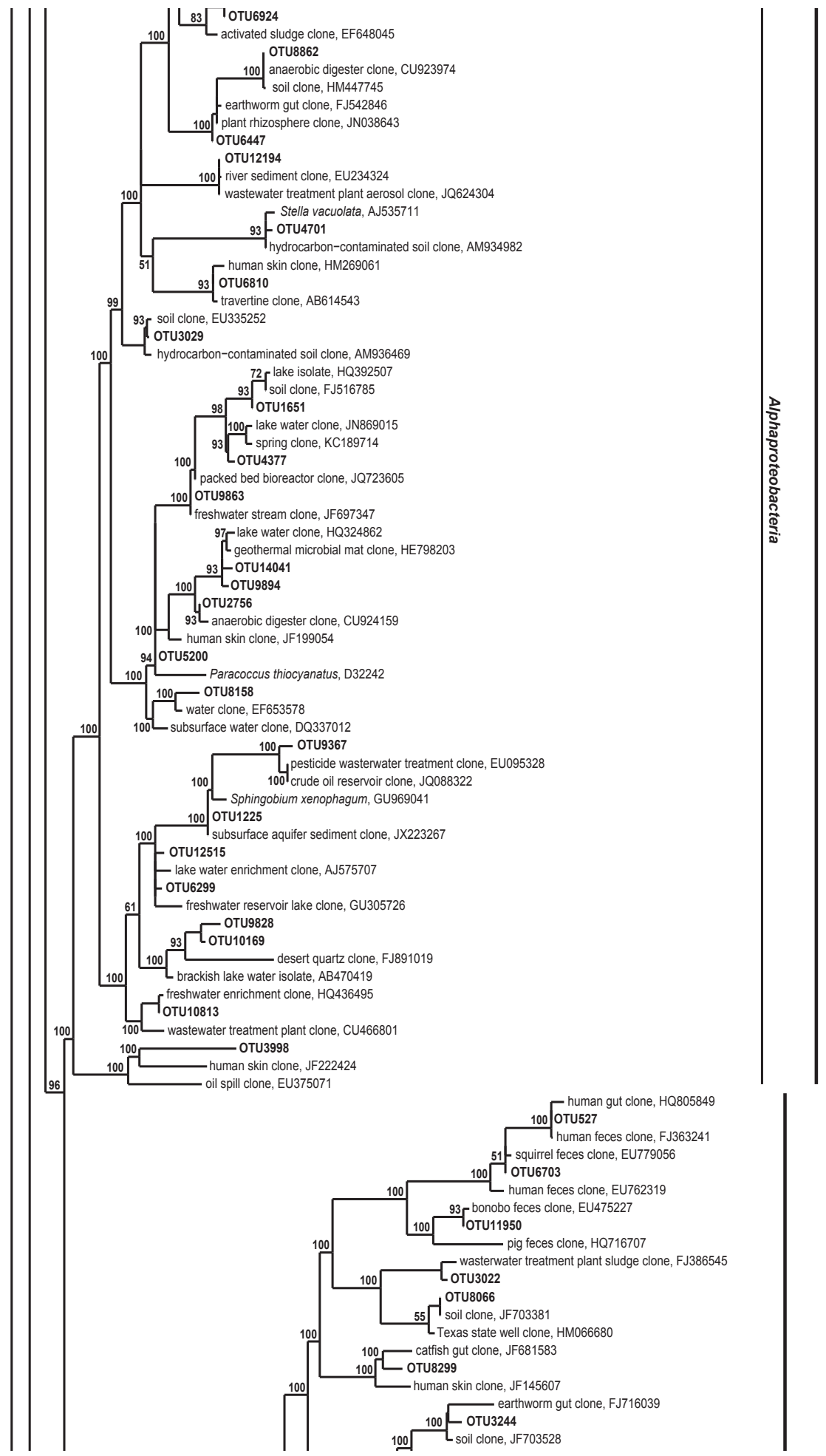

Figure S3.3. 


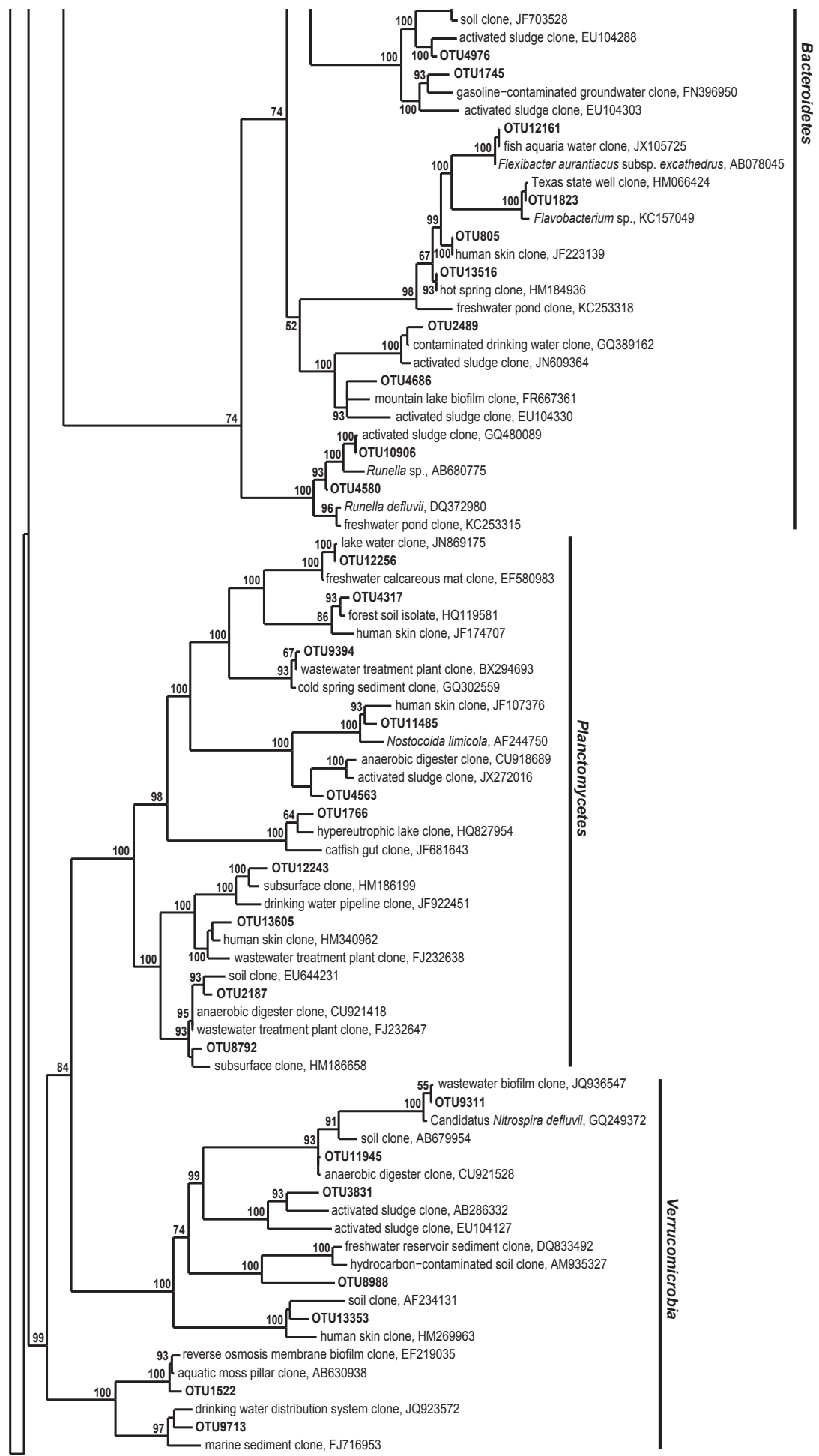

Figure S3.3. 

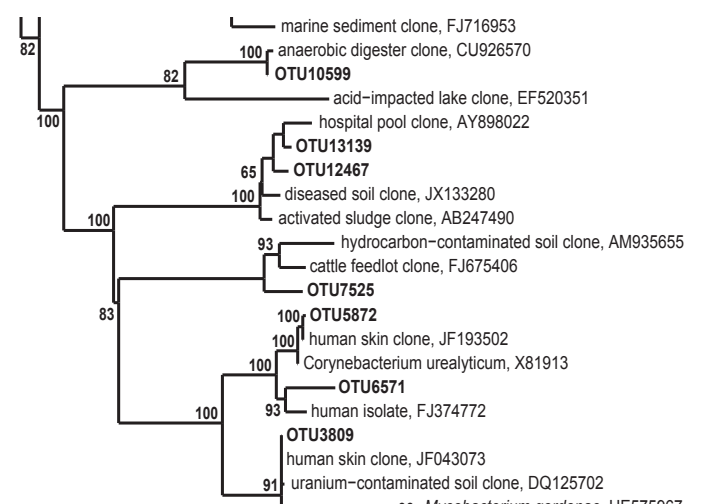

93. Mycobacterium gordonae, HE575967 ${ }_{100}^{93}$ OTU12537

$82 \longrightarrow$ hospital shower water clone, FJ382617

$68 \longrightarrow$ Mycobacterium komossense, X55591 OTU9738

${ }_{100}-$ Mycobacterium farcinogenes, X55592

OT wastewater isolate, AB773246

${ }_{90}{ }_{93}^{93}$ wastewater

93

- wetland isolate, FJ006915

OTU4790

100 river sediment isolate, EU167977

93. OTU12238

Anamox bioreactor clone, JX875885

${ }_{59}$ Mycobacterium sp., AF408993

$98 \longrightarrow$ OTU7180

- hospital shower water clone, FJ382219

93 Gordonia rhizosphera, AB004729

${ }_{93} \stackrel{93}{\text { OTU13780 }}$

L Gordonia polyisoprenivorans, HM195273

mangrove isolate, EF538739

93 OTU1005

89 Rhodococcus phenolicus, AM933579

Rhodococcus ruber, AB750389

100 OTU6755

oil-contaminated soil isolate, HM134277

$100-13$, rhizosphere isolate, JQ396591

OTU6761

$100 \rightarrow-$ intertidal sediment isolate, JX428894

Nocardia sp. isolate, JN896612

93 OTU11875

Nocardia takedensis, AB158277

100 plant isolate, FJ262979

93 OTU10161

Nocardia thailandica, JF797320

Nocardia asteroides, BAFO01000006

OTU7898

93 soil isolate, EU119253

99

\section{isolate, JN869461}

Figure S3.3. Bayesian phylogram of selected 16S rRNA gene sequences from this study (in bold) and closely related reference sequences. The numbers above or below the branches correspond to posterior probability (PP) values of the Bayesian analysis. PP values $<50$ are not indicated. The scale bar corresponds to the mean number of nucleotide substitutions per site.

OTU2028





\section{Chapter 4}

Steering the gut microbiome of tilapia larvae with microbial diets

is
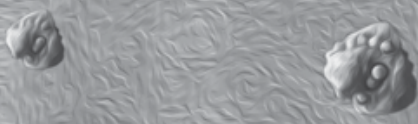

This chapter has been submitted for publication as:

Christos Giatsis, Marc Verdegem, Joost van Loo, Bram Rohaan, Johan Verreth, Hauke Smidt, Javier Ramiro-Garcia, Detmer Sipkema (2016). Steering the gut microbiome of tilapia larvae with microbial diets. 
Fish live and share their environment with microorganisms. The autochthonous microbiota of fish mostly originates from those present in their feed and water. However, the fish gut seems to be selective for the establishment of certain species, while at the same time being hostile to others. Despite the significant effort in defining the forces that impact the assembly of gut microbial communities, the underlying mechanisms attributed to the impact of the environment over the host selectivity are still poorly understood. In the present study the role of feed microbiota on gut communities of tilapia larvae was investigated. Fish were fed three experimental diets incorporated with sludge produced under aerobic, methanogenic or denitrifying conditions. We performed 16S rRNA gene sequence-based comparisons between gut microbial communities from different treatments and we associated them with the ones present in feed and water. Gut microbiota shared a much higher number of OTUs with microbiota in sludge-based feeds than with water, resulting in distinct gut communities between treatments. This finding implies that the tilapia gut microbiota has a certain plasticity, which makes it amenable to interventions. Nevertheless, in spite of observed changes in microbiota composition between treatments, a persistent core gut OTUs was maintained in all treatments. 


\subsection{Introduction}

The establishment of the microbial community in the gut is an essential part of the development in humans and animals, including fish (O'Hara and Shanahan, 2006; Ley et al., 2008; Sommer and Bäckhed, 2013; Lepage et al., 2012). Fish live and share the environment with dense microbial communities. This close association in the aquatic environment causes externally exposed body surfaces of fish, including the gut, to be quickly colonized after hatching or mouth opening (Reitan et al., 1998; Verschuere et al., 2000; Hansen and Olafsen, 1999). The digestive tract provides different (micro) habitat niches, resulting in the colonization of diverse microbiota (Sommer and Bäckhed, 2013; Hansen and Olafsen, 1999).

The autochthonous microbiota of fish originates from the environment. As fish produce axenic eggs, fish larvae have their first contact with microbes right after hatching (Verschuere et al., 2000). Legacy effects attributed to environmental factors, such as maternal influences, are known to affect the establishment of the gut microbiota in fish (Keskin et al., 1994; Olafsen, 2001; Sullam et al., 2012). However, the gut environment is selective for the establishment of certain species from the environment and the forces governing host selection are poorly understood.

In fish larvae, the selection of certain microbes in the gut has been suggested to be host-dependant (Li et al., 2012b). For instance, difference in gut microbiota between fish species sharing the same environment might be related to differences in the anatomy, immunology, feeding behaviour and physiology. In fish, host-selective capabilities were revealed in axenic zebrafish (Danio rerio), which received a faecal transplant derived from mice. The implanted mouse community subsequently shifted towards a state resembling a native zebrafish community (Rawls et al., 2006). In addition, zebrafish originating from the wild shared a core gut microbiota with those reared in captivity, demonstrating a host-specific microbial community in the gut (Roeselers et al., 2011). Also Wong et al. (2013) found that rainbow trout (O. mykiss) raised at different densities and fed different diets shared a core gut microbiota (Wong et al., 2013).

In a recent study, Giatsis et al. (2015) showed that feed microbiota had a very low impact on gut microbial communities. However, in that study a commercial pelleted feed was used. This suggests that perhaps feed microbial composition and/or load was not appropriate for a successful establishment of feed bacteria in the gut. Therefore, in this study three different microbial diets were prepared based on $1 \%$ inclusion (on dry matter basis) of sludge derived from aerobic, methanogenic or denitrifying reactors. Nile tilapia larvae were fed with a regular diet (i.e. control) or with the sludge-based diets and the effect of diet microbiota on the gut communities was assessed. All larvae were reared within the same system and shared the same water source in order to avoid variation between treatments due to differences in water microbiota. 
We hypothesized that if gut microbial composition is strongly shaped by selective pressures in the gut, then similar gut microbial communities should develop in all dietary treatments. Contrary, if the gut microbial community is strongly shaped by the composition of the microbial community present in the diet, this will result in a significantly different gut microbiota between treatments.

\subsection{Materials and Methods}

\subsubsection{Ethics statement}

The experiment was performed in accordance with relevant guidelines and approved by the Ethical Committee of Wageningen University for animal experiments (Project Name: Promicrobe; Registration code: 2012050b).

\subsubsection{Sludge and experimental diets preparation}

Three different types of sludge were produced in triplicate under aerobic, methanogenic and denitrifying conditions in customized reactors. Three sealable glass bottles (20 $L$ volume) with integrated electric heaters $(100 \mathrm{~W})$ were filled with $10 \mathrm{~L}$ tap water for each treatment. Magnetic stir bars were placed inside the bottles (450 rpm) to ensure solids suspension. In the aerobic sludge treatment, an air diffusor (air supply $5 \mathrm{~L} \mathrm{~h}^{-1}$ ) was placed to ensure oxygen-rich conditions. Both methanogenic and denitrifying reactors received $\mathrm{N}_{2}$ gas $\left(0.1 \mathrm{~L} \mathrm{~h}^{-1}\right)$. In the denitrifying reactors, $\mathrm{NaNO}_{3}$ was added to maintain the nitrate concentration above $200 \mathrm{mg} \mathrm{L}^{-1}$. The $\mathrm{pH}$, temperature, redox potential and nitrate concentration were measured daily and concentration of total ammonia nitrogen (TAN), nitrite and dissolved oxygen (DO) were measured weekly with a pH/Oxi 340i electrode (WTW GmbH, Weilheim, Germany) and the Merck Nitrate, Ammonium and Nitrite Test, respectively.

The sludge reactors were inoculated daily with faecal matter originating from a Recirculating Aquaculture System (RAS). The faecal matter was produced in RAS stocked with adult Nile tilapia fed a commercial diet, harvested with a sedimentation cone and stored into a glass bottle kept in ice to minimize the bacterial breakdown of collected matter. Approximately $1350 \mathrm{~mL}$ of faeces were collected daily at 8:45 AM, left standing undisturbed for solids to settle, collected by siphoning, manually mixed for $30 \mathrm{~s}$ and then equally divided over the nine sludge-reactors. For the diets preparation, sludge from the nine reactors was sampled daily and sludge-based diets were freshly prepared as described below. 


\subsubsection{Sludge incorporated diet production}

In total four experimental diets were tested. The three microbial diets consisted of three different types of sludge mixed with a commercial tilapia feed. The commercial feed was also used as the control treatment without sludge incorporation (Skretting Gemma Wean M0.5, 300-500 $\mu \mathrm{m}, 58 \%$ crude protein, $17 \%$ crude fat, $10 \%$ ash, $0.6 \%$ fibre, $1.3 \%$ phosphorus). The aerobic, methanogenic and denitrifying sludge was mixed with the commercial diet at $1 \%$ inclusion level on dry matter basis. The sludge suspension was first pipetted from the reactors and filtered through $0.2 \mu$ m (type GTTP) membrane filters (Millipore - Isopore ${ }^{\mathrm{TM}}$ ) by a vacuum pump for 1 minute. Subsequently, the retained sludge was collected and mixed with the commercial feed to create a solid paste. The control diet was the same commercial tilapia feed but instead of sludge, autoclaved water was used to form a paste of similar consistency as the sludge-based diets. After the preparation of each diet and the first feeding of the day, the remaining feed was sealed in an airtight bag (for the methanogenic and denitrifying diet, the oxic headspace was replaced by $\mathrm{N}_{2}$-gas) and stored at $5{ }^{\circ} \mathrm{C}$ until the next feeding of the day. The experimental diets were prepared on a daily basis.

\subsubsection{Experimental animals and rearing conditions}

A flow-through system (FTS) consisted of a buffer tank (200 L effective volume) and four holding tanks (i.e. four treatments) were used. In FTS, water flows continuously in the fish tanks and the outlet tank water is discarded without being recirculated. In such a way, cross transfer of feed particles, faecal matter and bacteria between treatments was avoided. No replicate FTS were used due to the focus of the experiment on the MC microbial communities composition in the gut of individual fish. Our previous research (Giatsis et al., 2014) revealed no differences in MC gut microbial communities composition between individuals reared in different tanks when the same water source was used.

One batch of full sibling Nile tilapia (Oreochromis niloticus) larvae was produced at the CARUS-Aquatic Research Facility (Wageningen UR). One hundred-5-days-post-fertilization (dpf) larvae were stocked in each tank. Feeding started at $9 \mathrm{dpf}$ (referred as day 0 ) and was continued for 16 days. Larvae were fed four times a day to apparent satiation for 30 minutes at 9:00, 11:30, 14:00 and 16:30. Water temperature was set to $28^{\circ} \mathrm{C}$, water exchange rate at $1 \mathrm{~L} \mathrm{~h}^{-1}$ and aeration inside the tanks was supplied at $5 \mathrm{~L} \mathrm{~h}^{-1}$. The photoperiod was set to 12 hours light/dark intervals.

Water quality parameters were maintained at safe levels for Nile tilapia larvae ( $\mathrm{pH}$ 8.1-8.6, temperature $28^{\circ} \mathrm{C}, \mathrm{TAN}<0.03 \mathrm{mg} \mathrm{L}^{-1}, \mathrm{NO}_{2}-\mathrm{N}<0.1 \mathrm{mg} \mathrm{L}^{-1}$ and $\mathrm{DO}>6 \mathrm{mg} \mathrm{L}^{-1}$ ). Temperature, dissolved oxygen and $\mathrm{pH}$ were measured daily with a $\mathrm{pH} / \mathrm{Oxi} 340 \mathrm{i}$ electrode (WTW GmbH, Weilheim, Germany). The $\mathrm{NO}_{3^{\prime}}$ TAN and $\mathrm{NO}_{2}$ concentrations were determined with a Merck Nitrate, Ammonium and Nitrite Test, respectively. 
The sampling scheme for microbiota analysis of gut, water, feed and sludge on sampling day 0,8 and 16, is provided in Table S4.1. Details regarding DNA isolation from gut, water, feed and sludge samples have been previously described elsewhere (Giatsis et al., 2014) and are available as supplementary material with the online version of this paper (Appendix).

\subsubsection{PCR and Illumina sequencing}

For 16S rRNA gene-based microbial composition profiling, barcoded amplicons from the V4 region of $16 \mathrm{~S}$ rRNA genes were generated by PCR using the $515 \mathrm{~F}$ and $806 \mathrm{R}$ primers (Caporaso et al., 2010). Seventy different barcodes were used per library in which the forward and reverse primer of one sample always carried the same barcode (Table S4.2). Primer sequence and barcode were separated by a 2-nucleotide linker sequence (GA for 515F and CG for 806R). Extracted DNA was diluted to a concentration of $20 \mathrm{ng} \mathrm{hL}^{-1}$ based on Nanodrop readings. PCR was performed using a GS0001 thermocycler (Gene Technologies, Braintree, United Kingdom). The PCR mix (50 $\mu \mathrm{L}$ final volume) contained $10 \mu \mathrm{L}$ of $5 \times \mathrm{HF}$ buffer (Finnzymes, Vantaa, Finland), $1 \mu \mathrm{L} 10 \mathrm{mM}$ (each nucleotide) PCR-grade Nucleotide Mix (Roche Diagnostic GmbH, Mannheim, Germany), $0.5 \mu \mathrm{L}$ of Phusion hot start II HighFidelity DNA polymerase (2U/ $\mu \mathrm{L}$ ) (Finnzymes), $1 \mu \mathrm{L} 10 \mu \mathrm{M}$ barcoded primer 515F (Biolegio BV, Nijmegen, The Netherlands), $1 \mu \mathrm{L} 10 \mu \mathrm{M}$ barcoded primer 806R, $36.5 \mu \mathrm{L}$ nuclease-free water and $1 \mu \mathrm{L} 20 \mathrm{ng} / \mu \mathrm{L}$ template DNA. Duplicate PCR reactions and a negative control, containing $1 \mu \mathrm{L}$ nuclease-free water, were run for every sample. PCR was performed under the following conditions: $98^{\circ} \mathrm{C}$ for $30 \mathrm{~s}$ to activate the polymerase, followed by 25 cycles consisting of denaturation at $98^{\circ} \mathrm{C}$ for $10 \mathrm{~s}$, annealing at $56^{\circ} \mathrm{C}$ for $10 \mathrm{~s}$, and elongation at $72^{\circ} \mathrm{C}$ for $10 \mathrm{~s}$, and a final extension at $72^{\circ} \mathrm{C}$ for $7 \mathrm{~min}$. Five $\mu \mathrm{L}$ of the PCR products were analyzed by $1 \%(w / v)$ agarose gel electrophoresis in the presence of $1 \times S Y_{B R}{ }^{\circledast}$ Safe (Invitrogen, Carlsbad, CA, USA). PCR products were purified using the High Pure PCR Cleanup Micro Kit (Roche Diagnostics) according to manufacturer's instructions and eluted in $10 \mu \mathrm{L}$ TE buffer. The DNA concentration in the PCR products was measured with the Qubit ${ }^{\circledR} 2.0$ Fluorometer (LifeTechnologies, Carlsbad, USA) and 70 barcoded PCR products were mixed per library using 200 ng DNA per sample. Four times $20 \mu \mathrm{L}$ per library were run on a 1\% agarose gel at $100 \mathrm{~V}$ for 45 minutes. The bands visible at approximately $300 \mathrm{bp}$ were excised from the gel and purified using the Zymoclean Gel DNA Recovery kit (Zymo Research) according to the manufacturer's instructions and eluted in $7 \mu \mathrm{L}$ nuclease-free water. Subsequently the library DNA was precipitated. First $0.1 \mathrm{~L}$ volume of $3 \mathrm{M}$ sodium acetate $(\mathrm{pH}$ 5.2) was added. Second, 3 volumes (calculated including the added sodium acetate) of $95 \%$ ethanol were also added. The library DNA was incubated overnight at $4^{\circ} \mathrm{C}$ before centrifugation at $13,400 \mathrm{~g}$ for $30 \mathrm{~min}$ at $4^{\circ} \mathrm{C}$. The supernatant was discarded and the pellet was rinsed with $400 \mu \mathrm{L} 70 \%$ ethanol and centrifuged for 15 minutes at $4^{\circ} \mathrm{C}$. Subsequently, the ethanol was discarded and the library DNA was dried in a vacuum centrifuge for $45 \mathrm{~s}$. The pellet was re-suspended in $16.5 \mu \mathrm{L}$ TE (1 $\mathrm{mM}$ Tris- $\mathrm{HCl}, 0.1 \mathrm{mM}$ EDTA, pH 8.0) and left for $1 \mathrm{~h}$ at $4^{\circ} \mathrm{C}$. 
The DNA concentration was measured with a Qubit ${ }^{\circledR} 2.0$ Fluorometer (Life Technologies, Carlsbad, USA) and $1 \mu \mathrm{L}$ was checked on a $1 \%$ agarose gel. Nucleotide sequences were generated using an Illumina HiSeq2000 sequencer at GATC-Biotech, Konstanz, Germany. Raw sequence data were deposited in GeneBank at NCBI under accession number SRP062676.

The pipeline and the parameters described in Ramiro-Garcia et al. (2016) were employed to convert the raw DNA sequence data to taxonomically labelled operational taxonomic unities (OTUs).

\subsubsection{Data handling and statistical analysis}

Microbial community data were expressed as relative abundance of all OTUs in each sample. Bray Curtis similarity between samples was calculated based on square root transformed data. Non metric multi-dimensional scaling (nMDS) was performed to represent the samples in a low dimensional space in a way that relative distances of all points were in the same rank order as the relative Bray Curtis dissimilarities of the samples. All statistical analyses were performed by using the multivariate statistical software package Primer V6 (Primer-E Ltd, Plymouth, UK).

To visualize shared OTUs between gut microbiota from different treatments and days, a network analysis was performed. Physical samples and OTUs are shown as nodes where each OTU is connected to each physical sample in which it was found. The group attributed layout, as implemented in Cytoscape 3.0.2 was used to cluster the OTUs in the network based on presence/absence data (Saito et al., 2012). Shared and unique OTUs of water gut or feed microbial communities were visualized with Venn diagrams. Venn diagrams were constructed by using the VENNY program without applying area proportionality to the 4-set diagrams (Oliveros, 2007).

\subsection{Results}

\subsubsection{Production of the different sludge types}

The aim of our study was to investigate whether gut microbial composition is strongly shaped by selective pressures in the gut and to which extent different microbial diets are able to modify those communities during early life development in fish.

Physicochemical parameters of the sludge reactors were significantly different among treatments. Redox values (mean $\pm \mathrm{SD}$ ) were highly negative in methanogenic $(-434.05 \mathrm{mV}$ $\pm 147.83)$, around zero in the denitrifying and positive in the aerobic reactors $(64.82 \mathrm{mV}$ 
\pm 32.79). Nitrate concentration was lower in the denitrifying and higher in both aerobic and methanogenic sludge reactor. Higher TAN was observed in the methanogenic and denitrifying reactors and highest nitrite was observed in the denitrifying ones (Table 4.1).

Table 4.1. Performance of reactors during sludge production under aerobic, methanogenic and denitrifying conditions. Table (ANOVA and Tukey's post-hoc test) shows mean values ( $n=3, \pm S D=$ standard deviation) of the measured parameters for each treatment during 23 weeks culture period (including the pre-experimental period). In each row, different superscripts indicate significant difference $(P<0.05)$. T: Temperature; Redox: Reduction oxidation; TAN: Total ammonia nitrogen; $\mathrm{NO}_{2}-\mathrm{N}$ : Nitrite nitrogen; $\mathrm{NO}_{3}-\mathrm{N}$ : Nitrate nitrogen; DO: Dissolved oxygen.

\begin{tabular}{lccc}
\hline Parameters & Aerobic & Methanogenic & Denitrifying \\
\hline $\mathbf{T}\left({ }^{\circ} \mathbf{C}\right)$ & $28.3 \pm 1.2^{\mathrm{a}}$ & $29.3 \pm 0.7^{\mathrm{c}}$ & $28.7 \pm 0.7^{\mathrm{b}}$ \\
$\mathbf{p H}$ & $8.5 \pm 0.3^{\mathrm{b}}$ & $7.8 \pm 0.6^{\mathrm{a}}$ & $8.7 \pm 0.7^{\mathrm{c}}$ \\
Redox (mV) & $64.8 \pm 32.8^{\mathrm{c}}$ & $-434.1 \pm 147.8^{\mathrm{a}}$ & $-2.3 \pm 146.7^{\mathrm{b}}$ \\
$\mathbf{N O}_{\mathbf{3}}\left(\mathbf{m g ~ L}^{-1}\right)$ & $118.5 \pm 116.6^{\mathrm{b}}$ & $6.7 \pm 29.1^{\mathrm{a}}$ & $181.0 \pm 87.6^{\mathrm{c}}$ \\
$\mathbf{T A N}_{\left(\mathbf{m g ~ L}^{-1}\right)}$ & $1.9 \pm 4.0^{\mathrm{a}}$ & $55.6 \pm 28.2^{\mathrm{b}}$ & $63.3 \pm 32.9^{\mathrm{b}}$ \\
$\mathbf{N O}_{\mathbf{2}}\left(\mathbf{m g ~ L}^{-1}\right)$ & $0.3 \pm 0.4^{\mathrm{a}}$ & $0.9 \pm 2.4^{\mathrm{a}}$ & $4.1 \pm 3.1^{\mathrm{b}}$ \\
$\mathbf{D O}\left(\mathbf{m g ~ L}^{-1}\right)$ & $5.5 \pm 2.3^{\mathrm{b}}$ & $0.1 \pm 0.2^{\mathrm{a}}$ & $0.6 \pm 0.7^{\mathrm{a}}$ \\
\hline
\end{tabular}

Microbial communities of the sludge differed considerably between the three reactor types. The high incorporation of sludge into the tilapia diets ( $99 \%$ on dry matter) resulted in differences of diet microbiota among treatments. Feed along with sludge samples clustered together according to the four dietary treatments and there were no large temporal changes observed over time (Figure 4.1). However, temporal variation of the microbial communities in the control diet (commercial feed) was higher than in the sludge based diets.

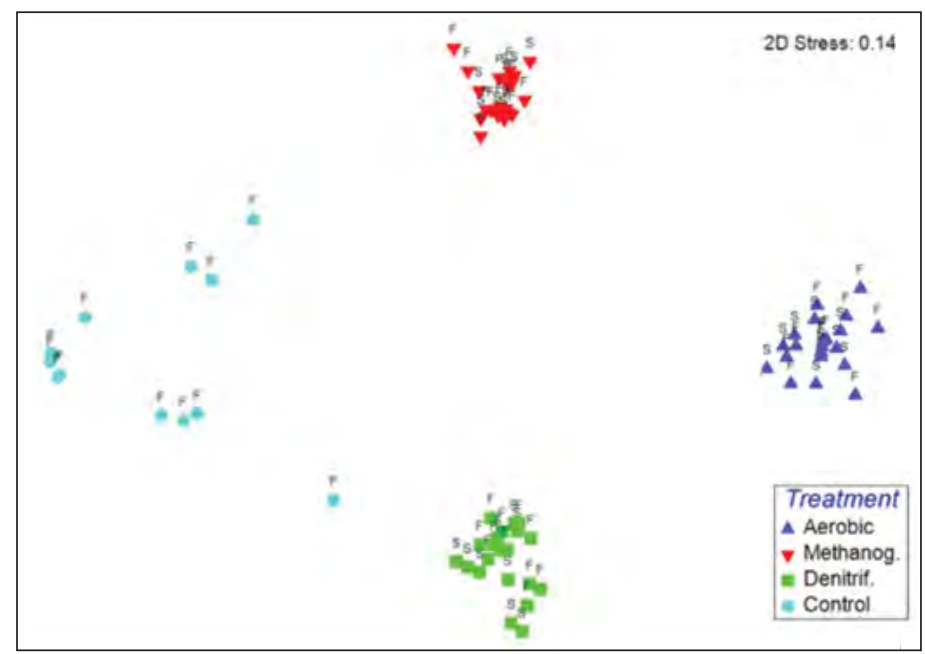

Figure 4.1. Non metric dimensional scaling ( $\mathrm{nMDS}$ ) of feed and sludge microbiota from the four dietary treatments. Each point represents a sludge (S) or feed microbiota (F) sample from days 0, 8 and 16. Beta-diversity is based on Bray Curtis after square root transformation of relative abundance data. Sludge was sampled in triplicate and feed samples in quadruplicate on each sampling day. Aerobic, Methanog., Denitrif., \& Control: Aerobic, methanogenic, denitrifying and control treatment. Stress value is reported for the two dimensions and is indicative of the quality of fit of the data into the plot. 
Regarding the unique and shared OTUs of the four diets, Venn diagrams showed that very few bacteria were shared between different diets and not even one OTU was present in all diets during day 16 (Figure 4.2).

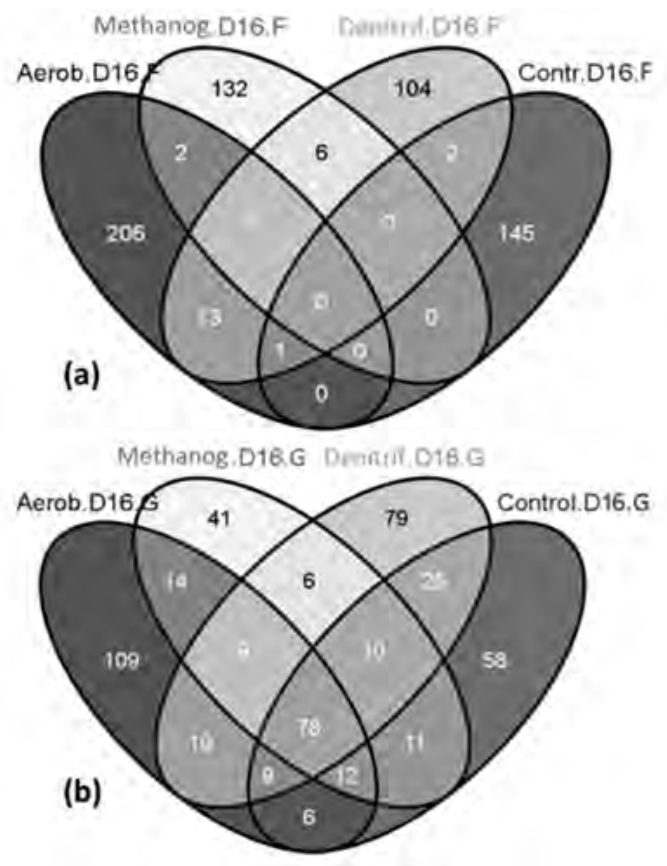

Figure 4.2. Venn diagram showing the number of shared and unique OTUs on day 16. Comparisons are made for (a) feed or (b) gut microbiota between the four treatments. Number of reads in all samples was normalized to the minimum number of reads found in one of the samples (cut off: 9719 reads) to allow a fair comparison between them. Aerob.G.16, Methanog.G.16, Denitrif.G.16 \& Control.G.16: Gut microbiota OTUs in the aerobic, methanogenic, denitrifying and control treatment G. \& F.: Gut and feed samples respectively; D16: Sampling day 16.

\subsubsection{The effect of microbial diets on gut microbiota}

Differences in the diet-associated microbiota were to a large extent observed also in the fish gut on both day 8 and 16 (Figure 4.1). Those differences were more prominent on day 16 with aerobic and methanogenic treatments clustering separately and denitrifying and control treatments overlapping (Figure 4.3). Dissimilarity of gut microbial communities due to dietary treatment was mostly attributed to the newly appeared OTUs of day 8 and 16 which instead were absent from the initial microbiota of the larvae. 
Gut MC on Day 8

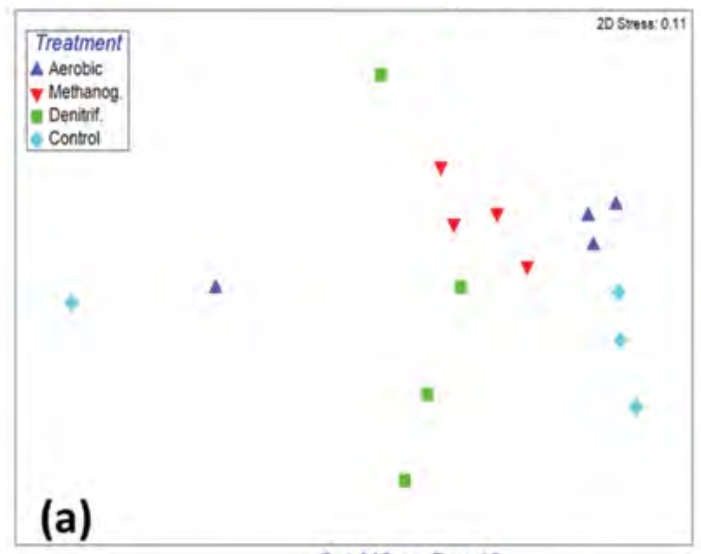

Gut MC on Day 16

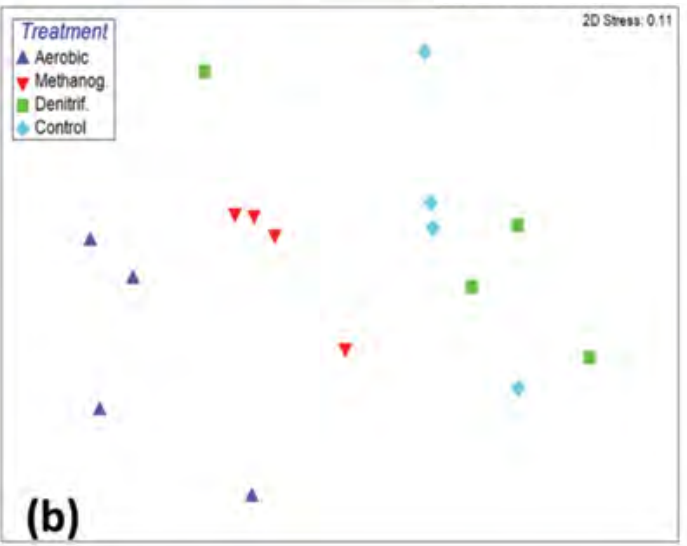

Figure 4.3. Non metric dimensional scaling (nMDS) of gut microbiota from individuals fed four different diets (complete dataset). Each point represents the gut microbiota of one individual. Plots are based on Bray Curtis distance after square root transformation of relative abundance data. Aerobic, Methanog., Denitrif., \& Control: Aerobic, methanogenic, denitrifying and control treatment on day 8 (a) and day 16 (b). Stress values are reported for the two dimensions and are indicative of the quality of fit of data into the plot.

In total, 119 to 141 new OTUs appeared in the gut of larvae in the aerobic, methanogenic and denitrifying treatment on day 8, and 121 and 173 OTUs on day 16 respectively. Interestingly, in most cases, the newly appeared gut OTUs seem to have originated from the diet, whereas in the control treatment, from the water (Table 4.2). 
Table 4.2. Numbers of gut OTUs appeared on either day 8 or 16 which were below threshold levels in the fish gut on day 0. Gut: Indicates the number of newly appeared OTUs in the gut; Water \& Feed: Indicate the number of the newly appeared gut OTUs also present in water or feed on either day 8 or 16 . Values in parenthesis show the $\%$ relative abundance of those OTUs in the gut, water or feed samples respectively on either day 8 or 16 .

\begin{tabular}{ccccc}
\hline \multirow{2}{*}{ Treatment } & Day & $\begin{array}{c}\text { Newly appeared } \\
\text { Gut OTUs }\end{array}$ & \multicolumn{2}{c}{ Newly appeared gut OTUs found only in } \\
\cline { 4 - 5 } & & $122(12.5 \%)$ & Water & Feed \\
\hline Aerobic & & $141(21.7 \%)$ & $22(11.5 \%)$ & $30(36.7 \%)$ \\
Methanogenic & 8 & $119(47.1 \%)$ & $6(1.3 \%)$ & $36(28.6 \%)$ \\
Denitrifying & & $98(13.9 \%)$ & $24(8.9 \%)$ & $44(34.2 \%)$ \\
Control & & $173(43.7 \%)$ & $9(1.1 \%)$ & $4(4.6 \%)$ \\
Aerobic & & $121(34.3 \%)$ & $14(8.6 \%)$ & $58(27.6 \%)$ \\
Methanogenic & \multirow{2}{*}{16} & $156(50.3 \%)$ & $11(8.6 \%)$ & $13(6.1)$ \\
Denitrifying & & $140(33.7 \%)$ & $13(10.2 \%)$ & $19(17.3 \%)$ \\
Control & & & &
\end{tabular}

At the end of the experiment, not a single OTU above threshold levels was shared among all four diets. However, 78 common OTUs were present in the fish gut regardless of the dietary treatment (Figure 4.3a \& b). From those, 39 and 26 highly abundant OTUs found on day 8 and 16 respectively, were already present in the gut of the initial population at day 0 (Figure S4.1). For this reason, we hypothesized that dietary effects on gut microbiota could have partly been masked by the persistent OTUs originating from the initial population (Figure 4.4). 


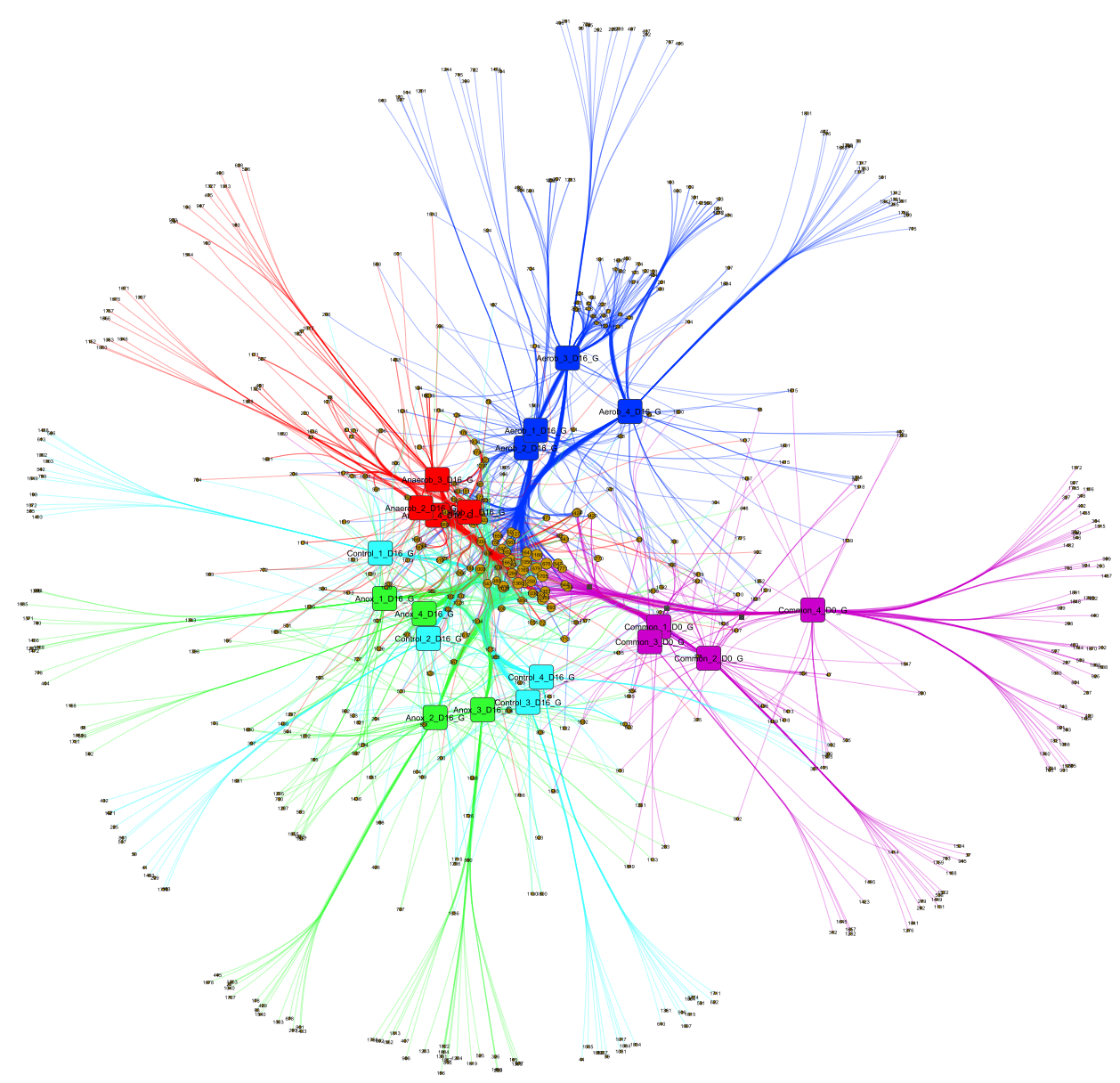

Figure 4.4. Network of shared and unique gut OTUs between all treatments on day 16 and the initial gut microbiota (Day 0). The network is color-coded by: treatment (Aerobic: blue; Methanogenic: red; Denitrifying: green; Control: cyan and initial population: purple). Sample nodes (squares) are oversized to be distinguished from OTU nodes (orange circles). Size of OTU nodes represent assignments of OTUs to sample nodes based on the amount of samples that were shared with (small nodes are shared with less samples compared to larger ones). Orange OTUs in the middle of network indicate the core OTUs found in all treatments and were also member of the initial gut microbiota.

Therefore, to obtain better insight into the changes induced by the diet, the gut OTUs, which were present in the initial population and persisted throughout the experiment, were removed from the dataset. A new dataset (subset) was plotted based on renormalized percentage relative OTU abundance. Removal of the core OTUs resulted in more pronounced differences between gut microbiota from the four dietary treatments and particularly on day 16 (Figure 4.5a \& b). 
Gut MC on Day 8

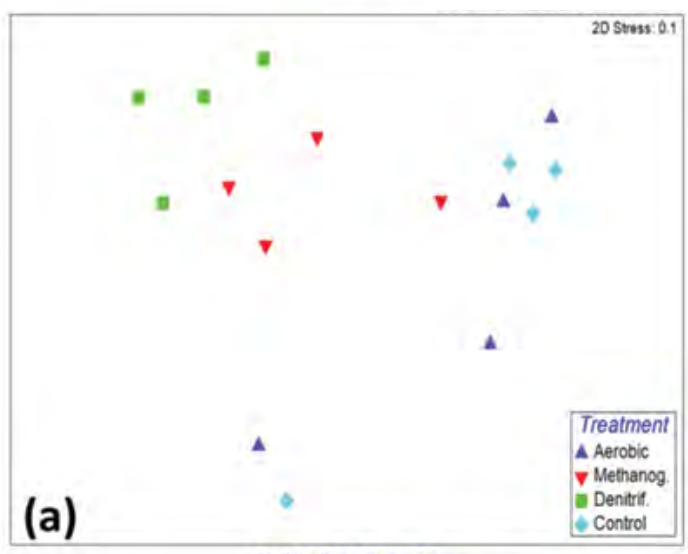

Gut MC on Day 16

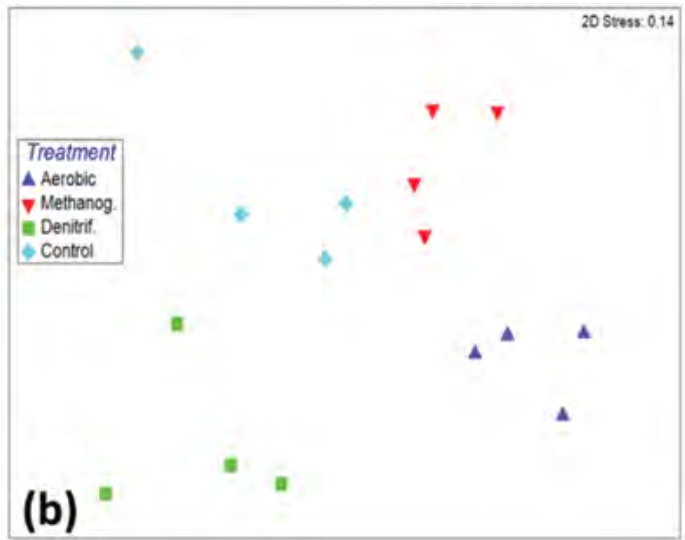

Figure 4.5. Non metric dimensional scaling (nMDS) of gut microbiota from individuals fed four different diets (reduced dataset). Each point represents the gut microbiota of one individual. Plots are based on Bray Curtis distance after square root transformation of relative abundance data. In total 26 OTUs shared between all treatments and days, and which were also present in the gut of the initial population (day 0 ), have been removed from the dataset. Aerobic, Methanog., Denitrif., \& Control: aerobic, methanogenic, denitrifying and control treatment on day 8 (a) and day 16 (b). Stress values are reported for the two dimensions and are indicative of the goodness of fit of data into the plot. 


\subsubsection{The predominant and core OTUs of the gut}

A heatmap of the most predominant gut OTUs (full dataset) on day 16 was created based on OTU relative abundance data (Figure 4.6). OTU 1416 (member of genus Ralstonia) was the most abundant OTU of the aerobic and methanogenic treatment on that day and the most abundant OTU of the initial gut microbiota (about 60\%) and day 8 . Another predominant gut OTU on day 16 was \#676 (member of the genus Rhodanobacter) that was present in all treatments but surprisingly absent from both water and diet microbiota. Rhodanobacter was already present on day 0 and relatively highly abundant in all treatments at day 8 .

The most predominant gut OTU of the denitrifying treatment at day 16 was \#1850 (member of the genus Cetobacterium), which, although it was also found in the denitrifying sludge-diet, was absent from all samples of the other treatments and days. The next most abundant OTU of the denitrifying treatment was a member of the genus Lactococcus (OTU 1680), which was again absent from all other treatments on that day.

In the control treatment, \#1032 (member of the genus Aeromonas) was the most predominant gut OTU on day 16 . However, the relative abundance of this OTU differed between replicate samples indicating the high interindividual variation within the control treatment regarding predominant bacteria. Aeromonas was not present, neither in the water nor in the diet during that day. Other highly abundant OTUs of the control treatment were: OTU 589 (member of the genus Rhizobium), OTU 584 (member of the genus Hyphomicrobium) and OTU 676 (member of the genus Rhodanobacter).

In total 26 gut OTUs from the initial population were encountered in the gut of all treatments and days. Those OTUs accounted for $79 \%$ of the total OTU relative abundance on day 0 and represent a large fraction of the initial colonization of the fish gut prior to the dietary treatments (Figure S4.1). On day 16, those initial predominant OTUs accounted for a large proportion ( 43 to $54 \%$ ) of the total OTU relative abundance in the gut of aerobic and methanogenic treatment. In the denitrifying and control treatment their abundance was lower though (37\%). The lower relative abundance of shared (core) OTUs in the denitrifying and control treatment was mostly attributed to the decrease over time in the relative abundance of OTU 1416 (member of genus Ralstonia) in these treatments. Other predominant shared OTUs of day 8 and 16 were 676 and 679 (member of the genus Rhodanobacter) as well as 1056 and 1166 (members of the genus Halomonas) (Figure 4.6). 


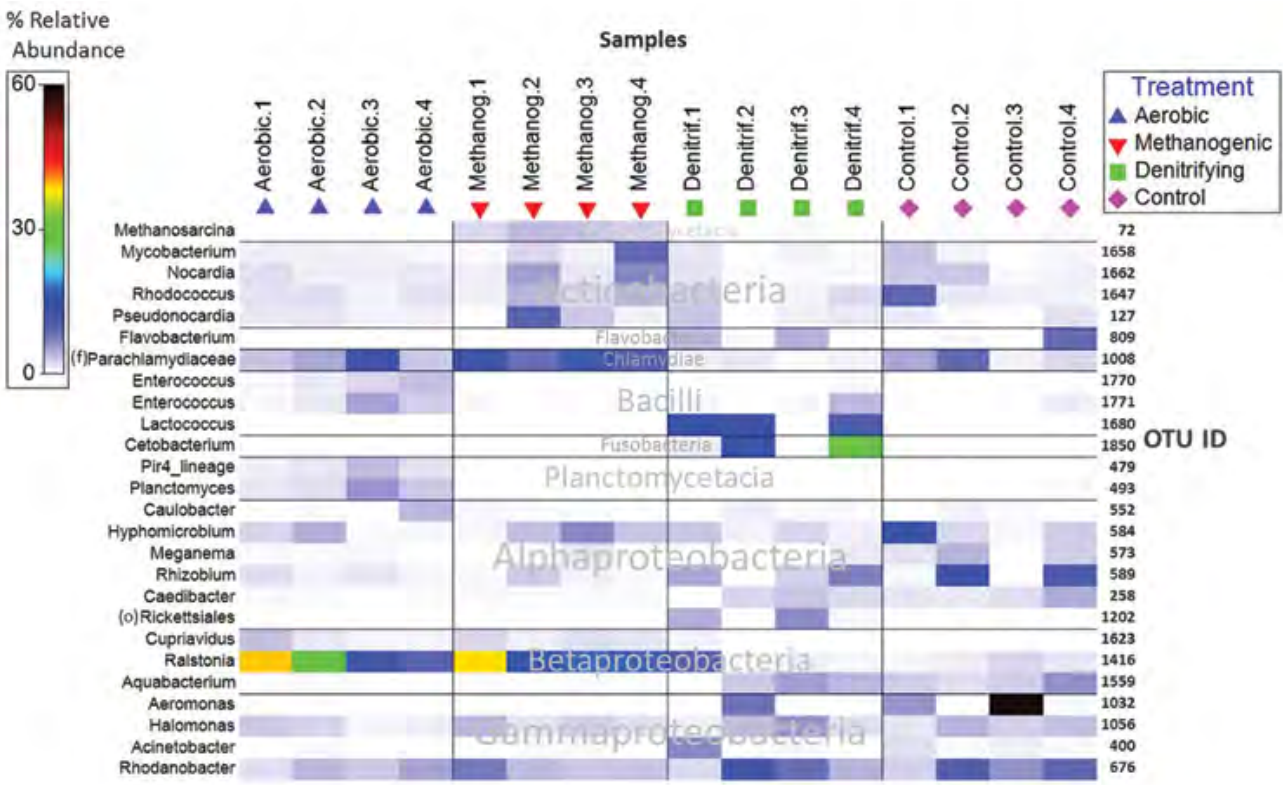

Figure 4.6. Heatmap of the five most predominant gut OTUs on day 16 in each replicate sample in the four treatments. Colours are proportional to the increasing percent relative OTU abundance (from white: lower, to red: higher) within each sample. Each column in the heatmap represents a sample, and each row represents an OTU. Columns are clustered by treatment, whereas the rows are clustered in accordance with the taxonomy of each OTU on class level. Aerobic, Methanogenic, Denitrifying \& Control 1-4: Four replicate gut samples from each of the four dietary treatments on day 16. Taxonomy (left column) indicates the genus of each OTU (right column) unless otherwise stated; (f): family, (o): order. OTUs are taxonomically clustered on class level as indicated in the middle of the heatmap.

\subsection{Discussion}

Bacteria associated with water and feed are two of the main environmental inoculum sources for gut microbiota in fish. Whilst water starts to affect the fish gut microbiota immediately after mouth opening (Reitan et al., 1998), intrusion of microbes through feeding becomes important later (Ringo and Birkbeck, 1999; Hansen and Olafsen, 1999). Bakke et al. (2013) demonstrated that the gut microbiota of cod larvae is more strongly influenced by microbiota present in the water rather than by the microbiota associated with feed (Bakke et al., 2013). In a previous study, we confirmed a high and consistent correlation between water and gut microbiota of tilapia larvae whereas less than $1 \%$ of all OTUs was shared between gut and feed (Giatsis et al., 2015). However, our present results show that bacteria associated to the diet lead to alterations in gut microbiota and that these changes are more pronounced in the sludge-based diets rather than in the control. Contrarily to previous studies (Bakke et al., 2013; Giatsis et al., 2015), gut shared a much higher number of OTUs with the feed than with the water. However, despite marked 
differences in the gut communities, the effect of the tested sludge-based diets was not sufficient to substantially modify a large core microbiota. These core gut OTUs were shared among all samples regardless the dietary treatment, and at the same time were already highly abundant in the gut of newly hatched larvae; already before the first feeding.

The concept of core gut microbiota has been proposed not only for humans (Turnbaugh and Gordon, 2009; Turnbaugh et al., 2009a), but recently also for fish. A study conducted in rainbow trout (Oncorhynchus mykiss) raised under different densities and diet composition, provided evidence for a core microbiota (Wong et al., 2013). Furthermore, Roeselers et al found that a core-microbiota was shared between wild zebrafish (Danio rerio) shared and zebrafish reared in captivity in different geographical locations (Roeselers et al., 2011). This suggests that host-specificity for particular microbial species is modulated by selective pressures within the host gut. In the present study, it was found that gut microbiota of tilapia larvae shared a large core microbiota regardless of the dietary treatments (Figure 4.4). Moreover, the 26 core OTUs that together made up 36 to $54 \%$ of the total relative abundance in the fish gut of all treatments, accounted for almost $80 \%$ of the total relative abundance on day 0 (Table S4.1). In this regard, tilapia core microbiota of this study can be defined as the group of OTUs encountered in all fish and sampling days (regardless the diet) and at the same time were also present in the gut of the initial population prior to the dietary treatments.

Regarding the phylogenetic affiliation of the core OTUs, our analysis revealed a diverse set of Proteobacteria (especially alpha and gamma), Actinobacteria and Firmicutes. The higher the taxonomic level of comparison and the diversity within each taxon, the less meaningful the definition of core microbiota becomes in terms of functionality. For this reason, our comparative phylogenetic analysis of gut microbiota in different individuals was performed on OTU level. Among the most dominant core OTUs found in this study were members of the genera Ralstonia, Halomonas and Rhodanobacter. In fish, the genus Ralstonia (OTU 1416) has been previously found in the gut of seabass (Carda-Diéguez et al., 2014), rainbow trout (Kim et al., 2007; Navarrete et al., 2012; Pond et al., 2006), yellow catfish (Wu et al., 2010), zebrafish (Rawls et al., 2004) but also in shrimp (Durand et al., 2010). The genus Halomonas (OTUs 1056 \& 1166) has been reported in the gut of Arctic charr (Ringo et al., 2006), Atlantic cod (Fjellheim et al., 2011), Midas cichlids (Franchini et al., 2014), queen conch (Carrascal et al., 2014), and Artemia brine shrimp (Tkavc et al., 2011; Riddle et al., 2013), and also in Atlantic salmon gills (Steinum et al., 2009). The "core microbiota" observed here has not been previously reported as core in tilapia. However, many of the bacterial genera found here (Mycobacterium, Nocardia, Rhodococcus, Halomonas, and Ralstonia) were also encountered in our previous study (Giatsis et al., 2016a) suggesting that tilapia specificity for particular microbial taxa could be modulated by selective pressures within the host. 
Lack of shared predominant species across studies in tilapia does not necessarily imply difference in functionality of these communities within the gut. The concept of functional redundancy suggests that functional diversity of an ecosystem is additive when species are complementary, or decreases, when species are to some extent functionally redundant (Bell et al., 2005). Metagenomics analysis of gut communities in fish could increase our knowledge of gene content and genetic variability in this microbiome. However, metatransciptomics analysis of the gut microbiota would be necessary to reveal whether functional patterns in the fish gut are complementary or redundant, and whether or not, these patterns match the genetic variability of the microbiome.

Mycobacteria are obligate aerobic, Gram-positive, non-spore forming, non-motile and prevalent in soil and water bacteria and have been previously reported in tilapia gut (Zhou et al., 2009; He et al., 2012, 2011, 2010), although not as core. The genus Rhodococcus has been reported in the gut of many fish species (Kim et al., 2007; Sanchez et al., 2012; Spanggaard et al., 2000; Huber et al., 2004). Hyphomicrobium was among the dominant genera in the gut of catfish (McDonald et al., 2012). Representatives of Hyphomicrobium are ubiquitous in nature, residing in soils, as well as fresh and wastewaters. Members of the genus have been shown to be associated with turbot and brook charr skin microbiota (Mudarris and Austin, 1988; Montes et al., 1999; Boutin et al., 2014), or water/filter microbiota (Sugita et al., 2005; Austin, 1983; Auffret et al., 2013). These findings suggest that some of the commonly found genera of this study could be involved in major metabolic functions in the fish gut. However, the observed differences in abundance of core genera across studies could to a certain degree pertain to the environmental microbiota (i.e. availability of certain bacteria), dietary interventions or other experimental treatments.

The tilapia larvae studied here were maintained under identical rearing conditions prior to the start of the experiment. The presence of the shared species is likely to be attributed to an early colonization that greatly diminished the impact of the experimental manipulations, thus reinforcing the early life imprinting hypothesis. The fact that core species of this study were not defined as core in our previous study might be related to the early exposure of larvae to different environmental microbiota (maternal or water) between the two experiments. Early microbial colonization has been suggested to have a long lasting effect on the host through early-life imprinting (Sommer and Bäckhed, 2013). The control over the composition of the gut microbiota, which is exerted by the maternal microbiota or by the exposure to the maternal environment inoculum, has been suggested in humans (Ley, 2010; Benson et al., 2010; Tannock et al., 1990) and animals (Ley et al., 2005; Zhao et al., 2013). However, the extent and the mechanisms underlying such a control, remain the major unanswered questions in gut microbiology (Flint et al., 2007). Contrary to terrestrial animals, which inherit a part of their initial microbiota through contact with the mother, fish usually spawn axenic eggs in the water (Verschuere et al., 2000). Nevertheless, the Nile tilapia is a known maternal mouth-brooder among the 
cichlids. In such context, an initial contact of eggs with the maternal mouth microbiota suggests a strong influence on initial microbial colonization of the egg surfaces, before being transferred to incubators for hatching. The early life of fish larvae in intensive rearing systems takes place in incubators with both egg debris and hatching eggs. This suggests that both egg and incubator water microbial communities are important factors in establishing the indigenous larval microbiota (Olafsen, 2001; Keskin et al., 1994) and it might explain the differences in the core microbial species between our two studies.

Besides the close phylogenetic relationships of the larvae and the legacy effects during hatching (both inheritance from mother and environment), another reason for the large number of shared OTUs among fish of this study could be the common water source among treatments. A common water source might limit the variability in microbial community composition among the larvae and between treatments. In a previous study, we demonstrated that tilapia larvae shared a large number of OTUs that originated from the water when fish were fed the same diet but exposed to different water sources (Giatsis et al., 2014). However, in our current study tilapia larvae shared only a few OTUs with water, implying that the system water was not the main drive of large fraction of bacteria shared between fish from all treatments. Yet in a few cases, some of the most abundant gut OTUs (i.e. OTU 1416 on day 16 and OTU 1031 on day 8) were also encountered in the water, though in very low abundance. This finding suggests that either there is selection of some rare water bacteria in the fish gut, and/or that, predominant bacteria in the gut end up in the water through defecation. However the latter is not a valid hypothesis for bacteria present in the feed, which means that shared bacteria between gut and feed could only originate from the feed.

Although several genera predominated the gut microbiota in all treatments, analysis of the accessory microbiota (excluding the 26 core gut OTUs shared between day 0,8 and 16), revealed that differences between gut microbial communities matched differences in dietary microbial communities. Besides the core OTUs, some of the most predominant OTUs in the gut were members of the genera Cetobacterium, Lactococcus, Aeromonas, Rhizobium, Hyphomicrobium and Aquabacterium. Both Cetobacterium (OTU 1850) and Lactococcus (OTU 1680) dominated the gut from larvae receiving the denitrifying treatment diet and were found in low abundance in the methanogenic treatment dietfed fish. These genera were also present in the feed of these two treatments on day 8 and 16 , but they were not detected in any other treatment or in the initial gut. As these two genera were not detected in the water either, it is presumed that these bacteria entered the gut through the feed. Members of the genus Cetobacterium (mostly C. somerae (Finegold et al., 2003) have been previously identified in the gut of several fish species such as catfish (Wu et al., 2012a; Di Maiuta et al., 2013), zebrafish (Roeselers et al., 2011), as well as goldfish, carp and in our previous study in tilapia larvae (Giatsis et al., 2015). The presence of this bacterium in a number of freshwater fishes suggests the importance of 
these microbes as members of the fish gut community. The presence and abundance of the genus Cetobacterium in the methanogenic and denitrifying feed and gut, confirms the preference of these organisms for oxygen depleted habitats. Other gut OTUs which possibly originated from the feed are members of the family Planctomycetaceae (OTU 199 and 493), which were detected only in the gut and feed of the aerobic treatment. Most members of this family are chemo-organotrophs and obligate aerobes and tend to be slow-growers compared to other bacteria (Op den Camp et al., 2007). Both on day 8 and 16, OTU 72 (genus Methanosarcina) was highly abundant in the gut and among the most dominant OTUs in the feed of the methanogenic treatment. Methanosarcina is a genus of methane producing euryarchaeotes. These organisms are anaerobic methanogens that produce methane using all three metabolic pathways for methanogenesis (Matarazzo et al., 2012). They have been reported in diverse environments such as in groundwater, anaerobic sludge reactors, freshwater lakes but also in human digestive tract and fish faeces (Ma et al., 2013; Angelakis et al., 2012; Samuel et al., 2007; Jones et al., 2008; Van Der Maarel et al., 1999; Dridi et al., 2011).

In a study with chicken, Yin et al. (2010), suggested that bacterial succession in the gut at a very early stage of life can be influenced by exposure to proper bacterial inocula, and that host selective pressures exert an additional role in colonization process. This statement brings out three interesting points: (a) steering gut microbiota with a microbial inoculum has higher chances to succeed during early life stages of animals compared to adult; (b) the word "proper" indicates the importance of microbial composition of the inoculum for a successful colonization and (c) regardless the previous two points, host selective pressure plays a major role in determining gut colonization.

Our data showed that the microbial composition of the sludge-based diets had corresponding effects on gut microbiota. In addition, fish gut microbiota shared more OTUs with the microbial diets, whereas fish fed the control diet shared more OTUs with water. Considering the short period of this study, one could speculate that by prolonging the experimental period, shared gut OTUs between treatments would disappear; or on the other hand that, if the administration of the experimental diets was terminated, the species composition and abundance of gut microbiota would return to an equilibrium among the treatments. These assumptions raise some very interesting considerations regarding the use of probiotics for steering fish gut microbiota. The main drawbacks of probiotic use are related to low survival and proliferation rate inside the fish gut (Gatesoupe, 1999; Grześkowiak et al., 2012). Important gaps in knowledge regarding selection criteria and colonization potential of single vs. multistrain probiotics (Pérez et al., 2010; Ramos et al., 2013), as well as competitive exclusion of the probiotic strain in the gut still exist (Martínez Cruz et al., 2012). Tackling such issues will require studies of model organisms where potentially confounding variables (host genotype) can be constrained by using for example inbred fish strains or clone lines. The use of microbial fecal transplants and/ 
or probiotics in gnotobiotic fish could help us explore the relative contributions of legacy versus habitat (including dietary components) in selecting a microbial community. This information will facilitate the development of safe and effective methods for manipulating gut microbiota composition to promote the health of humans and animals.

\section{Acknowledgements}

We are grateful to M. Ter Veld, H. Heilig and G. Bacanu for their contribution on samples collection and laboratory analysis. This work was funded by The European Community's Seventh Framework Program (FP7/2007-2013) under grant agreement no. 227197 Promicrobe "Microbes as positive actors for more sustainable aquaculture".

\section{Supplementary material}

Table S4.1. Sampling scheme with number of gut, water, sludge and feed samples taken for 16S rRNA gene sequencing analysis during the three experimental days. For determining the initial gut microbiota of the larvae, four gut samples were taken (not whole larvae) from the common batch before distributing larvae in the tanks and initiate feeding.

\begin{tabular}{|c|c|c|c|c|c|c|c|c|c|c|c|c|c|c|}
\hline & & \multicolumn{4}{|c|}{ Day 0} & \multicolumn{4}{|c|}{ Day 8} & \multicolumn{4}{|c|}{ Day 16} & \multirow[b]{2}{*}{ Total } \\
\hline \multicolumn{2}{|c|}{ Sample } & $\frac{0}{\frac{0}{0}}$ & 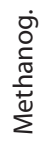 & 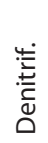 & 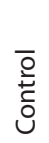 & $\frac{0}{\frac{0}{0}}$ & 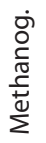 & 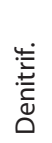 & 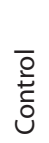 & $\frac{O}{\frac{0}{0}}$ & 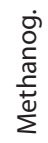 & 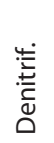 & 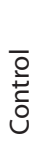 & \\
\hline \multicolumn{2}{|c|}{ Gut } & \multicolumn{4}{|c|}{4} & 4 & 4 & 4 & 4 & 4 & 4 & 4 & 4 & 36 \\
\hline \multirow{3}{*}{ Water } & Inlet & 0 & 0 & 0 & 0 & 1 & 1 & 1 & 1 & 1 & 1 & 1 & 1 & 8 \\
\hline & Tank & 1 & 1 & 1 & 1 & 1 & 1 & 1 & 1 & 1 & 1 & 1 & 1 & 12 \\
\hline & Outlet & 0 & 0 & 0 & 0 & 1 & 1 & 1 & 1 & 1 & 1 & 1 & 1 & 8 \\
\hline \multicolumn{2}{|c|}{ Sludge } & 3 & 3 & 3 & 0 & 3 & 3 & 3 & 0 & 3 & 3 & 3 & 0 & 27 \\
\hline \multicolumn{2}{|c|}{ Feed } & 4 & 4 & 4 & 4 & 4 & 4 & 4 & 4 & 4 & 4 & 4 & 4 & 48 \\
\hline \multicolumn{2}{|c|}{ Total } & \multicolumn{4}{|c|}{33} & \multicolumn{4}{|c|}{53} & \multicolumn{4}{|c|}{53} & 139 \\
\hline
\end{tabular}

Table S4.2. Metadata of samples included in the sequencing libraries. Seventy different barcodes were used per library in which the forward and reverse primer of one sample always carried the same barcode.

\begin{tabular}{lcccccccl}
\hline \#SampleID & $\begin{array}{c}\text { Barcode } \\
\text { Sequence }\end{array}$ & $\begin{array}{c}\text { Library } \\
\end{array}$ & $\begin{array}{c}\text { Forward } \\
\text { Sequence } \\
\text { length }\end{array}$ & $\begin{array}{c}\text { Reverse } \\
\text { Sequence } \\
\text { length }\end{array}$ & $\begin{array}{c}\text { Sample } \\
\text { Type }\end{array}$ & $\begin{array}{c}\text { Barcode } \\
\text { Number }\end{array}$ & Day Treatment \\
\hline Aerob.D0.W & AACCAGAA & 1 & 71 & 70 & WATER & 1 & 0 & Aerobic \\
Methanog.D0.W & AACCATGC & 1 & 71 & 70 & WATER & 2 & 0 & Methanogenic \\
Denitrif.DO.W & AACGGCGC & 1 & 71 & 70 & WATER & 3 & 0 & Denitrifying \\
Control.DO.W & AAGGAGCG & 1 & 71 & 70 & WATER & 4 & 0 & Control \\
\hline
\end{tabular}




\begin{tabular}{|c|c|c|c|c|c|c|c|c|}
\hline \#SampleID & $\begin{array}{l}\text { Barcode } \\
\text { Sequence }\end{array}$ & Library & $\begin{array}{l}\text { Forward } \\
\text { Sequence } \\
\text { length }\end{array}$ & $\begin{array}{l}\text { Reverse } \\
\text { Sequence } \\
\text { length }\end{array}$ & $\begin{array}{c}\text { Sample } \\
\text { Type }\end{array}$ & $\begin{array}{l}\text { Barcode } \\
\text { Number }\end{array}$ & Day & Treatment \\
\hline Aerob.inlet.D8.W & ACCAATTA & 1 & 71 & 70 & WATER & 5 & 8 & Aerobic \\
\hline Aerob.tank.D8.W & ACCGGAAT & 1 & 71 & 70 & WATER & 6 & 8 & Aerobic \\
\hline Methanog.inlet.D8.W & ACGCTCGC & 1 & 71 & 70 & WATER & 7 & 8 & Methanogenic \\
\hline Methanog.tank.D8.W & AGACCTGC & 1 & 71 & 70 & WATER & 8 & 8 & Methanogenic \\
\hline Denitrif.inlet.D8.W & AGCAGATT & 1 & 71 & 70 & WATER & 9 & 8 & Denitrifying \\
\hline Denitrif.tank.D8.W & AGCTTCGC & 1 & 71 & 70 & WATER & 10 & 8 & Denitrifying \\
\hline Control.inlet.D8.W & AGGTCGAA & 1 & 71 & 70 & WATER & 11 & 8 & Control \\
\hline Control.tank.D8.2.W & AGGTTATG & 1 & 71 & 70 & WATER & 12 & 8 & Control \\
\hline Aerob.inlet.D16.W & AGTCGCGC & 1 & 71 & 70 & WATER & 13 & 16 & Aerobic \\
\hline Aerob.tank.D16.W & AGTTATGC & 1 & 71 & 70 & WATER & 14 & 16 & Aerobic \\
\hline Methanog.inlet.D16.W & ATATCCGC & 1 & 71 & 70 & WATER & 15 & 16 & Methanogenic \\
\hline Methanog.tank.D16.W & ATGCCGGA & 1 & 71 & 70 & WATER & 16 & 16 & Methanogenic \\
\hline Denitrif.inlet.D16.W & ATGCGATT & 1 & 71 & 70 & WATER & 17 & 16 & Denitrifying \\
\hline Denitrif.tank.D16.W & САACCTCT & 1 & 71 & 70 & WATER & 18 & 16 & Denitrifying \\
\hline Control.inlet.D16.W & CAACGAGG & 1 & 71 & 70 & WATER & 19 & 16 & Control \\
\hline Control.tank.D16.W & CATAAGCG & 1 & 71 & 70 & WATER & 20 & 16 & Control \\
\hline Aerob.out.D8.W & CATGATGC & 1 & 71 & 70 & WATER & 21 & 8 & Aerobic \\
\hline Methanog.out.D8.W & CATTGCCA & 1 & 71 & 70 & WATER & 22 & 8 & Methanogenic \\
\hline Denitrif.out.D8.W & CCATGCGC & 1 & 71 & 70 & WATER & 23 & 8 & Denitrifying \\
\hline Control.out.D8.W & CCATTATG & 1 & 71 & 70 & WATER & 24 & 8 & Control \\
\hline Aerob.out.D16.W & CCGTACTA & 1 & 71 & 70 & WATER & 25 & 16 & Aerobic \\
\hline Methanog.out.D16.W & CCGTCTGC & 1 & 71 & 70 & WATER & 26 & 16 & Methanogenic \\
\hline Denitrif.out.D16.W & CGCAAGCT & 1 & 71 & 70 & WATER & 27 & 16 & Denitrifying \\
\hline Control.out.D16.W & CGGAAGAG & 1 & 71 & 70 & SLUDGE & 28 & 16 & Control \\
\hline Aerob.A.D0.S & CTAGGAGA & 1 & 71 & 70 & SLUDGE & 29 & 0 & Aerobic \\
\hline Aerob.B.D0.S & CTCAGCGC & 1 & 71 & 70 & SLUDGE & 30 & 0 & Aerobic \\
\hline Aerob.C.D0.S & CTCAGTCT & 1 & 71 & 70 & SLUDGE & 31 & 0 & Aerobic \\
\hline Methanog.A.D0.S & CTCGCGTA & 1 & 71 & 70 & SLUDGE & 32 & 0 & Methanogenic \\
\hline Methanog.B.D0.S & CTGCTGAA & 1 & 71 & 70 & SLUDGE & 33 & 0 & Methanogenic \\
\hline Methanog.C.D0.S & CTTGCGAG & 1 & 71 & 70 & SLUDGE & 34 & 0 & Methanogenic \\
\hline Denitrif.A.D0.S & CTTGGCCT & 1 & 71 & 70 & SLUDGE & 35 & 0 & Denitrifying \\
\hline Denitrif.B.D0.S & GAACCGTT & 1 & 71 & 70 & SLUDGE & 36 & 0 & Denitrifying \\
\hline Denitrif.C.D0.S & GAACGTAT & 1 & 71 & 70 & SLUDGE & 37 & 0 & Denitrifying \\
\hline Aerob.A.D8.S & GAACTAAG & 1 & 71 & 70 & SLUDGE & 38 & 8 & Aerobic \\
\hline Aerob.B.D8.S & GAAGCTCG & 1 & 71 & 70 & SLUDGE & 39 & 8 & Aerobic \\
\hline Aerob.C.D8.S & GAGTTATA & 1 & 71 & 70 & SLUDGE & 40 & 8 & Aerobic \\
\hline Methanog.A.D8.S & GATGAATG & 1 & 71 & 70 & SLUDGE & 41 & 8 & Methanogenic \\
\hline Methanog.B.D8.S & GATGATAA & 1 & 71 & 70 & SLUDGE & 42 & 8 & Methanogenic \\
\hline Methanog.C.D8.S & GATGCGCT & 1 & 71 & 70 & SLUDGE & 43 & 8 & Methanogenic \\
\hline Denitrif.A.D8.S & GCCAGGTT & 1 & 71 & 70 & SLUDGE & 44 & 8 & Denitrifying \\
\hline Denitrif.B.D8.S & GCCTTAAG & 1 & 71 & 70 & SLUDGE & 45 & 8 & Denitrifying \\
\hline Denitrif.C.D8.S & GCTAATCT & 1 & 71 & 70 & SLUDGE & 46 & 8 & Denitrifying \\
\hline Aerob.A.D16.S & GGAGCGCA & 1 & 71 & 70 & SLUDGE & 47 & 16 & Aerobic \\
\hline Aerob.B.D16.S & GGAGTATG & 1 & 71 & 70 & SLUDGE & 48 & 16 & Aerobic \\
\hline Aerob.C.D16.S & GGTACCAA & 1 & 71 & 70 & SLUDGE & 49 & 16 & Aerobic \\
\hline
\end{tabular}




\begin{tabular}{|c|c|c|c|c|c|c|c|c|}
\hline \#SampleID & $\begin{array}{l}\text { Barcode } \\
\text { Sequence }\end{array}$ & Library & $\begin{array}{l}\text { Forward } \\
\text { Sequence } \\
\text { length }\end{array}$ & $\begin{array}{l}\text { Reverse } \\
\text { Sequence } \\
\text { length }\end{array}$ & $\begin{array}{c}\text { Sample } \\
\text { Type }\end{array}$ & $\begin{array}{l}\text { Barcode } \\
\text { Number }\end{array}$ & Day & Treatment \\
\hline Methanog.A.D16.S & GGTAGAAT & 1 & 71 & 70 & SLUDGE & 50 & 16 & Methanogenic \\
\hline Methanog.B.D16.S & GTCCGCAA & 1 & 71 & 70 & SLUDGE & 51 & 16 & Methanogenic \\
\hline Methanog.C.D16.S & GTTAAGTT & 1 & 71 & 70 & SLUDGE & 52 & 16 & Methanogenic \\
\hline Denitrif.A.D16.S & GTTCTACG & 1 & 71 & 70 & SLUDGE & 53 & 16 & Denitrifying \\
\hline Denitrif.B.D16.S & TATTGCGC & 1 & 71 & 70 & SLUDGE & 54 & 16 & Denitrifying \\
\hline Denitrif.C.D16.S & TCAGCGAG & 1 & 71 & 70 & SLUDGE & 55 & 16 & Denitrifying \\
\hline Aerob.1.D0.F & TCATTCCG & 1 & 71 & 70 & FEED & 56 & 0 & Aerobic \\
\hline Aerob.2.D0.F & TCCGTATA & 1 & 71 & 70 & FEED & 57 & 0 & Aerobic \\
\hline Aerob.3.D0.F & TCCTCCGC & 1 & 71 & 70 & FEED & 58 & 0 & Aerobic \\
\hline Aerob.4.D0.F & TCGAATAA & 1 & 71 & 70 & FEED & 59 & 0 & Aerobic \\
\hline Methanog.1.D0.F & TCGATATT & 1 & 71 & 70 & FEED & 60 & 0 & Methanogenic \\
\hline Methanog.2.D0.F & TCTATTCG & 1 & 71 & 70 & FEED & 61 & 0 & Methanogenic \\
\hline Methanog.3.D0.F & TGACTCAA & 1 & 71 & 70 & FEED & 62 & 0 & Methanogenic \\
\hline Methanog.4.D0.F & TGATCTCA & 1 & 71 & 70 & FEED & 63 & 0 & Methanogenic \\
\hline Denitrif.1.D0.F & TGCCTGCG & 1 & 71 & 70 & FEED & 64 & 0 & Denitrifying \\
\hline Denitrif.2.D0.F & TGGTAATT & 1 & 71 & 70 & FEED & 65 & 0 & Denitrifying \\
\hline Denitrif.3.D0.F & TGGTATGA & 1 & 71 & 70 & FEED & 66 & 0 & Denitrifying \\
\hline Denitrif.4.D0.F & AACCAGAA & 2 & 71 & 70 & FEED & 1 & 0 & Denitrifying \\
\hline Control.1.D0.F & AACCATGC & 2 & 71 & 70 & FEED & 2 & 0 & Control \\
\hline Control.2.D0.F & AACGGCGC & 2 & 71 & 70 & FEED & 3 & 0 & Control \\
\hline Control.3.D0.F & AAGGAGCG & 2 & 71 & 70 & FEED & 4 & 0 & Control \\
\hline Control.4.D0.F & ACCAATTA & 2 & 71 & 70 & FEED & 5 & 0 & Control \\
\hline Aerob.1.D8.F & ACCGGAAT & 2 & 71 & 70 & FEED & 6 & 8 & Aerobic \\
\hline Aerob.2.D8.F & ACGCTCGC & 2 & 71 & 70 & FEED & 7 & 8 & Aerobic \\
\hline Aerob.3.D8.F & AGACCTGC & 2 & 71 & 70 & FEED & 8 & 8 & Aerobic \\
\hline Aerob.4.D8.F & AGCAGATT & 2 & 71 & 70 & FEED & 9 & 8 & Aerobic \\
\hline Methanog.1.D8.F & AGCTTCGC & 2 & 71 & 70 & FEED & 10 & 8 & Methanogenic \\
\hline Methanog.2.D8.F & AGGTCGAA & 2 & 71 & 70 & FEED & 11 & 8 & Methanogenic \\
\hline Methanog.3.D8.F & AGGTTATG & 2 & 71 & 70 & FEED & 12 & 8 & Methanogenic \\
\hline Methanog.4.D8.F & AGTCGCGC & 2 & 71 & 70 & FEED & 13 & 8 & Methanogenic \\
\hline Denitrif.1.D8.F & AGTTATGC & 2 & 71 & 70 & FEED & 14 & 8 & Denitrifying \\
\hline Denitrif.2.D8.F & ATATCCGC & 2 & 71 & 70 & FEED & 15 & 8 & Denitrifying \\
\hline Denitrif.3.D8.F & ATGCCGGA & 2 & 71 & 70 & FEED & 16 & 8 & Denitrifying \\
\hline Denitrif.4.D8.F & ATGCGATT & 2 & 71 & 70 & FEED & 17 & 8 & Denitrifying \\
\hline Control.1.D8.F & CAACCTCT & 2 & 71 & 70 & FEED & 18 & 8 & Control \\
\hline Control.2.D8.F & CAACGAGG & 2 & 71 & 70 & FEED & 19 & 8 & Control \\
\hline Control.3.D8.F & CATAAGCG & 2 & 71 & 70 & FEED & 20 & 8 & Control \\
\hline Control.4.D8.F & CATGATGC & 2 & 71 & 70 & FEED & 21 & 8 & Control \\
\hline Aerob.1.D16.F & CATTGCCA & 2 & 71 & 70 & FEED & 22 & 16 & Aerobic \\
\hline Aerob.2.D16.F & CCATGCGC & 2 & 71 & 70 & FEED & 23 & 16 & Aerobic \\
\hline Aerob.3.D16.F & CCATTATG & 2 & 71 & 70 & FEED & 24 & 16 & Aerobic \\
\hline Aerob.4.D16.F & CCGTACTA & 2 & 71 & 70 & FEED & 25 & 16 & Aerobic \\
\hline Methanog.1.D16.F & CCGTCTGC & 2 & 71 & 70 & FEED & 26 & 16 & Methanogenic \\
\hline Methanog.2.D16.F & CGCAAGCT & 2 & 71 & 70 & FEED & 27 & 16 & Methanogenic \\
\hline Methanog.3.D16.F & CGGAAGAG & 2 & 71 & 70 & FEED & 28 & 16 & Methanogenic \\
\hline Methanog.4.D16.F & CTAGGAGA & 2 & 71 & 70 & FEED & 29 & 16 & Methanogenic \\
\hline
\end{tabular}




\begin{tabular}{|c|c|c|c|c|c|c|c|c|}
\hline \#SampleID & $\begin{array}{l}\text { Barcode } \\
\text { Sequence }\end{array}$ & Library & $\begin{array}{l}\text { Forward } \\
\text { Sequence } \\
\text { length }\end{array}$ & $\begin{array}{l}\text { Reverse } \\
\text { Sequence } \\
\text { length }\end{array}$ & $\begin{array}{c}\text { Sample } \\
\text { Type }\end{array}$ & $\begin{array}{l}\text { Barcode } \\
\text { Number }\end{array}$ & Day & Treatment \\
\hline Denitrif.1.D16.F & CTCAGCGC & 2 & 71 & 70 & FEED & 30 & 16 & Denitrifying \\
\hline Denitrif.2.D16.F & CTCAGTCT & 2 & 71 & 70 & FEED & 31 & 16 & Denitrifying \\
\hline Denitrif.3.D16.F & CTCGCGTA & 2 & 71 & 70 & FEED & 32 & 16 & Denitrifying \\
\hline Denitrif.4.D16.F & CTGCTGAA & 2 & 71 & 70 & FEED & 33 & 16 & Denitrifying \\
\hline Control.1.D16.F & CTTGCGAG & 2 & 71 & 70 & FEED & 34 & 16 & Control \\
\hline Control.2.D16.F & CTTGGCCT & 2 & 71 & 70 & FEED & 35 & 16 & Control \\
\hline Control.3.D16.F & GAACCGTT & 2 & 71 & 70 & FEED & 36 & 16 & Control \\
\hline Control.4.D16.F & GAACGTAT & 2 & 71 & 70 & FEED & 37 & 16 & Control \\
\hline Common.1.D0.G & GAACTAAG & 2 & 71 & 70 & GUT & 38 & 0 & Common \\
\hline Common.2.D0.G & GAAGCTCG & 2 & 71 & 70 & GUT & 39 & 0 & Common \\
\hline Common.3.D0.G & GAGTTATA & 2 & 71 & 70 & GUT & 40 & 0 & Common \\
\hline Common.4.D0.G & GATGATAA & 2 & 71 & 70 & GUT & 42 & 0 & Common \\
\hline Aerob.1.D8.G & GATGCGCT & 2 & 71 & 70 & GUT & 43 & 8 & Aerobic \\
\hline Aerob.2.D8.G & GCCAGGTT & 2 & 71 & 70 & GUT & 44 & 8 & Aerobic \\
\hline Aerob.3.D8.G & GCCTTAAG & 2 & 71 & 70 & GUT & 45 & 8 & Aerobic \\
\hline Aerob.4.D8.G & GCTAATCT & 2 & 71 & 70 & GUT & 46 & 8 & Aerobic \\
\hline Methanog.1.D8.G & GGAGCGCA & 2 & 71 & 70 & GUT & 47 & 8 & Methanogenic \\
\hline Methanog.2.D8.G & GGAGTATG & 2 & 71 & 70 & GUT & 48 & 8 & Methanogenic \\
\hline Methanog.3.D8.G & GGTACCAA & 2 & 71 & 70 & GUT & 49 & 8 & Methanogenic \\
\hline Methanog.4.D8.G & GGTAGAAT & 2 & 71 & 70 & GUT & 50 & 8 & Methanogenic \\
\hline Denitrif.1.D8.G & GTCCGCAA & 2 & 71 & 70 & GUT & 51 & 8 & Denitrifying \\
\hline Denitrif.2.D8.G & GTTAAGTT & 2 & 71 & 70 & GUT & 52 & 8 & Denitrifying \\
\hline Denitrif.3.D8.G & GTTCTACG & 2 & 71 & 70 & GUT & 53 & 8 & Denitrifying \\
\hline Denitrif.4.D8.G & TATTGCGC & 2 & 71 & 70 & GUT & 54 & 8 & Denitrifying \\
\hline Control.1.D8.G & TCAGCGAG & 2 & 71 & 70 & GUT & 55 & 8 & Control \\
\hline Control.2.D8.G & TCATTCCG & 2 & 71 & 70 & GUT & 56 & 8 & Control \\
\hline Control.3.D8.G & TCCGTATA & 2 & 71 & 70 & GUT & 57 & 8 & Control \\
\hline Control.4.D8.G & TCCTCCGC & 2 & 71 & 70 & GUT & 58 & 8 & Control \\
\hline Aerob.1.D16.G & TCGAATAA & 2 & 71 & 70 & GUT & 59 & 16 & Aerobic \\
\hline Aerob.2.D16.G & TCGATATT & 2 & 71 & 70 & GUT & 60 & 16 & Aerobic \\
\hline Aerob.3.D16.G & TCTATTCG & 2 & 71 & 70 & GUT & 61 & 16 & Aerobic \\
\hline Aerob.4.D16.G & TGACTCAA & 2 & 71 & 70 & GUT & 62 & 16 & Aerobic \\
\hline Methanog.1.D16.G & TGATCTCA & 2 & 71 & 70 & GUT & 63 & 16 & Methanogenic \\
\hline Methanog.2.D16.G & TGCCTGCG & 2 & 71 & 70 & GUT & 64 & 16 & Methanogenic \\
\hline Methanog.3.D16.G & TGGTAATT & 2 & 71 & 70 & GUT & 65 & 16 & Methanogenic \\
\hline Methanog.4.D16.G & TGGTATGA & 2 & 71 & 70 & GUT & 66 & 16 & Methanogenic \\
\hline Denitrif.1.D16.G & TTAGGATG & 2 & 71 & 70 & GUT & 67 & 16 & Denitrifying \\
\hline Denitrif.2.D16.G & AACCAGAA & 3 & 71 & 70 & GUT & 1 & 16 & Denitrifying \\
\hline Denitrif.3.D16.G & AACCATGC & 3 & 71 & 70 & GUT & 2 & 16 & Denitrifying \\
\hline Denitrif.4.D16.G & AACGGCGC & 3 & 71 & 70 & GUT & 3 & 16 & Denitrifying \\
\hline Control.1.D16.G & AAGGAGCG & 3 & 71 & 70 & GUT & 4 & 16 & Control \\
\hline Control.2.D16.G & ACCAATTA & 3 & 71 & 70 & GUT & 5 & 16 & Control \\
\hline Control.3.D16.G & ACCGGAAT & 3 & 71 & 70 & GUT & 6 & 16 & Control \\
\hline Control.4.D16.G & ACGCTCGC & 3 & 71 & 70 & GUT & 7 & 16 & Control \\
\hline
\end{tabular}



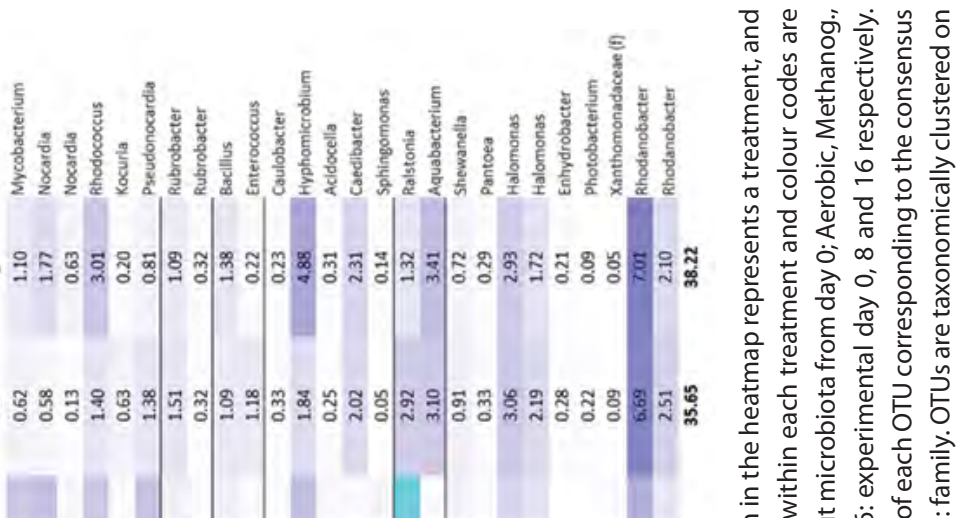

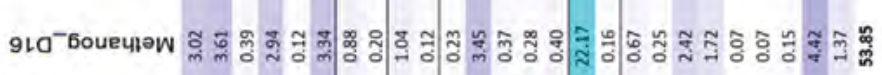

そ 3

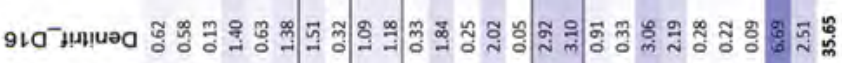

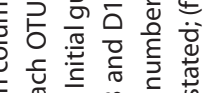

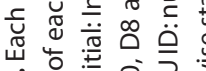

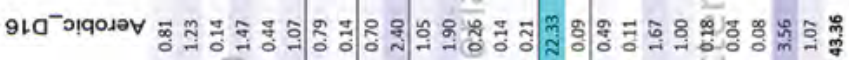

$\frac{\text { อ }}{\text { हू }}$

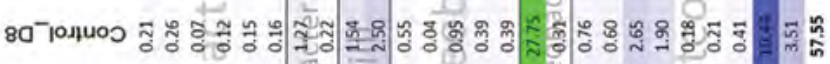

ทั่

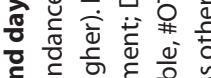

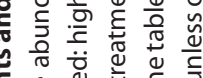

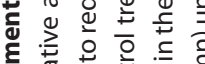

ह艹

。

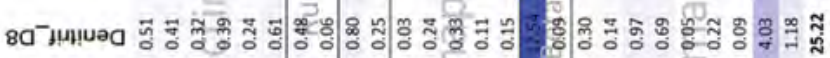

స

ฐ

范

\&

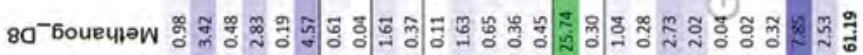

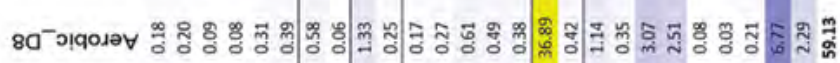

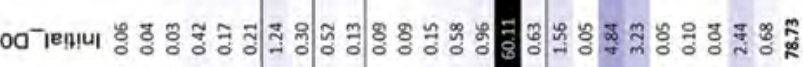

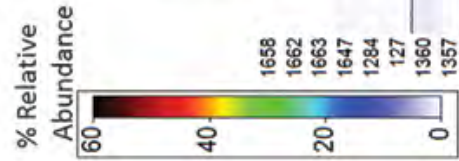

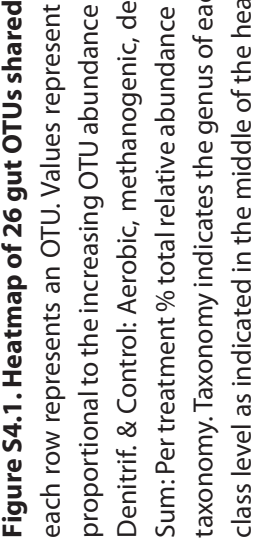




\section{Chapter 5}

\section{Probiotic legacy effects on gut microbial assembly in tilapia larvae}

i
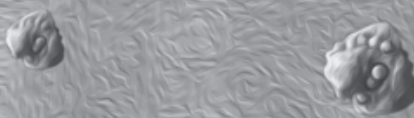

This chapter has been accepted for publication as:

Giatsis C, Ramiro-Garcia J, Abernathy J, Verreth J, Smidt H, Sipkema D, Verdegem M. (2016a). Probiotic legacy on gut microbial assembly in fish larvae. 
The exposure of fish to environmental free-living microbes and its effect on early colonization in the gut have been studied in recent years. However, little is known regarding how the host and environment interact to shape gut communities during early life. Here, we tested whether the early microbial exposure of tilapia larvae affects the gut microbiota at later life stages. The experimental period was divided into three stages: axenic, probiotic and active suspension. Axenic tilapia larvae were reared either under conventional conditions (active suspension systems) or exposed to a single strain probiotic (Bacillus subtilis) added to the water. Microbial characterization by Illumina HiSeq sequencing of $16 \mathrm{~S}$ rRNA gene amplicons showed the presence of $B$. subtilis in the gut during the seven days of probiotic application. Although $B$. subtilis was no longer detected in the guts of fish exposed to the probiotic after day 7 , gut microbiota of the exposed tilapia larvae remained significantly different from that of the control treatment. Compared with the control, fish gut microbiota under probiotic treatment was less affected by spatial differences resulting from tank replication, suggesting that the early probiotic contact contributed to the subsequent observation of low interindividual variation. 


\subsection{Introduction}

The gut microbiota influences a wide range of biological processes in humans (Rawls, 2007; Sekirov et al., 2010), domesticated terrestrial animals (Chaucheyras-Durand and Durand, 2010; McFall-Ngai et al., 2013) and fish (Nayak, 2010; Sullam et al., 2012). In fish, despite the significant contribution of several studies on gut microbiota, the current understanding of the functional significance of microbial fluctuations lags well behind that of terrestrial vertebrates. Apart from a few pathogens, host-microbe interactions in fish remain poorly understood. One reason is that the fish gut microbiota is dependent on the aquatic environment. Furthermore, compared with terrestrial animals that undergo embryonic development within an amnion, fish larvae are released into the water at an early ontogenetic stage, when their digestive tract is not yet fully developed and their immune system incomplete (Gatesoupe, 1999). Thus, the use of probiotics in aquaculture is particularly effective during early ontogenetic stages, where large mortalities are commonly observed.

Live microorganisms that confer a health benefit to the host have been demonstrated as useful in aquaculture (Pérez-Sánchez et al., 2014). Probiotics reduce infections caused by bacterial pathogens (Gatesoupe, 1999; Martínez Cruz et al., 2012) and have been successfully used as immunostimulants (Cain and Swan, 2010; Lepage et al., 2012; Song et al., 2014) and growth promoters in fish and shrimp (Pérez-Sánchez et al., 2014; Merrifield et al., 2010). However, probiotic strains often only transiently colonize the gut and quickly fall below detection limits (Gatesoupe, 1999; Grześkowiak et al., 2012; Skjermo et al., 2015; Robertson et al., 2000; Kim and Austin, 2006; Balcázar et al., 2007). For ingested bacteria to proliferate and persist within "resident" microbiota, these microorganisms must adapt to the environmental conditions inside the gut, such as nutrient availability, $\mathrm{pH}$ and digestive enzymes (Hansen and Olafsen, 1999). The ability of a probiotic strain to survive and successively proliferate in the gut after the cessation of probiotics administration is both host- and probiotic strain-dependent and is highly determined by the mode and duration of administration (through water or feed) (Pérez-Sánchez et al., 2014). Based on the present level of understanding, the colonization dynamics of fish gut microbiota remain largely stochastic and are affected by gut habitat (i.e., physiology, anatomy) and host genotype (Yan et al., 2012; Rawls et al., 2006; Navarrete et al., 2012). Yin et al. (2010) suggested that in newly hatched chicks, it is possible to steer gut microbiota by feeding bacterial diets (caecal inocula), leading to the development of distinct communities. In a recent study, we observed that tilapia larvae fed with different microbial diets (sludgebased) developed distinct gut microbiota, although all larvae also shared a large number of species (Giatsis et al., 2015). It is likely that those shared species resulted from larval contact with a common water source after hatching and prior to the first feeding. However, the host-specificity for a particular microbial species modulated by selective pressures within the host gut cannot be excluded. 
Bacillus subtilis is a Gram- and catalase-positive, rod-shaped, facultative, anaerobic and endospore-forming bacterium observed in air, water, soil and the gastrointestinal tract of humans and animals (Nakano and Zuber, 1998; Green et al., 1999; Hong et al., 2009; Tseng et al., 2009). Several Bacillus spp. (including B. subtilis) have been commonly used as probiotics in aquaculture, reflecting their antimicrobial activity against common fish and shrimp pathogens. The beneficial properties of these microbes primarily reflect immune system enhancement (phenoloxidase activity, phagocytic activity and clearance efficiency), competitive exclusion or antibacterial substance production (Wang et al., 2008; Liu et al., 2010; Balcázar and Rojas-Luna, 2007; Vaseeharan and Ramasamy, 2003; Aly et al., 2008; Kumar et al., 2006, 2008; Tseng et al., 2009; Salinas et al., 2005; Raida et al., 2003; Newaj-Fyzul et al., 2007). In addition, probiotic treatment with B. subtilis enhanced the growth and viability of beneficial lactic acid bacteria in the guts of humans and animals (Ngo Thi Hoa et al., 2000).

Prior to investigating the potential probiotic properties of B. subtilis in Nile tilapia, the scope of the present study was to assess the impact of the early microbial contact of tilapia larvae on the tilapia gut microbial assembly during later ontogenetic stages. We hypothesized that administration of the probiotic strain to fish larvae early in life, when the gut microbiota is still developing, enhances gut-colonization success and therefore leads to the development of distinct gut communities, even after the fish are exposed to conventional husbandry conditions in active suspension systems.

\subsection{Methods}

\subsubsection{Ethics statement}

This experiment was performed in accordance with Dutch regulations regarding the use of experimental animals and approved by the Ethical Committee of Wageningen University for animal experiments (Project Name: Promicrobe; Registration code: 2011076.c).

\subsubsection{Experimental Design}

The experimental period was divided into three stages: axenic, probiotic and active suspension. The first two stages were conducted under laboratory conditions, while the third stage was conducted under normal rearing conditions at the Aquatic Research Facility of CARUS, the Animal Experimental Facility of Wageningen University. The total experimental period was 28 days, which is considered sufficient for major ontogenetic changes to occur in tilapia until larvae enter the early juvenile stage (Fujimura and Okada, 2007). First, from two days post-fertilization, the eggs were reared under axenic conditions 
for seven days (Days 1 - 7). Subsequently, the axenic larvae were split into two groups. Half of the larvae was divided over three replicate active suspension tanks, i.e., C1-3 (from day 8 to 28 ), while the other half was divided over two probiotic chambers ( $\mathrm{P}-\mathrm{CH} 1$ and $\mathrm{P}-\mathrm{CH} 2$ ). Probiotic bacteria were supplied for seven consecutive days (days 8 - 14), after which the larvae from these probiotic chambers were divided over three active suspension tanks (P1-3), where these fish were further raised for another 14 days (day 15 - 28) (Figure 5.1). Throughout the text, “Control (C) treatment" refers to the axenic larvae directly transferred into xenic active suspension tanks, whereas "Probiotic $(P)$ treatment" refers to the axenic larvae initially exposed to the probiotic strain and subsequently transferred to active suspension tanks.

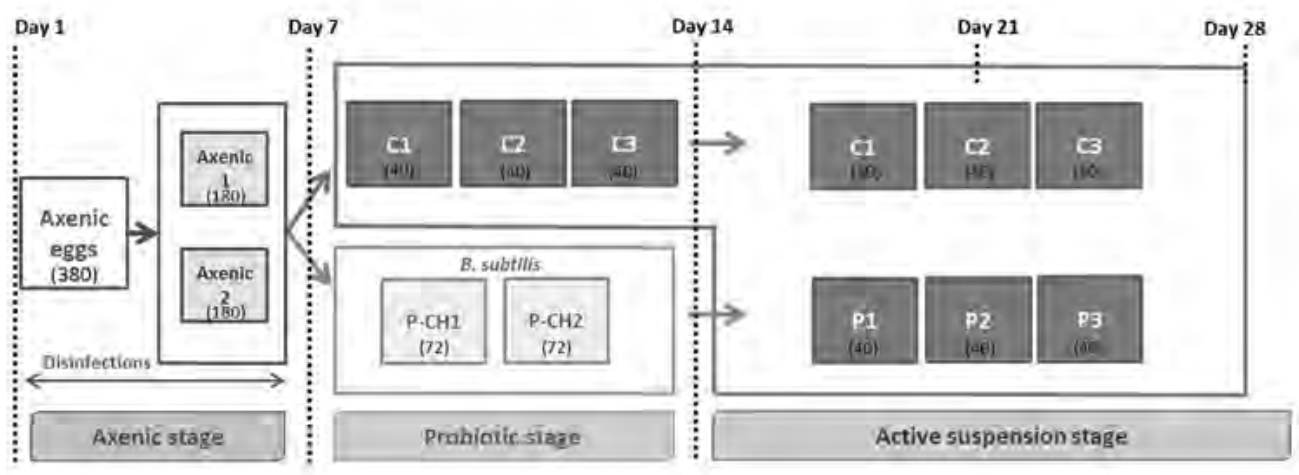

Figure 5.1. Experimental set-up during the 28-day experimental period. The period was divided into three different stages: Axenic, Probiotic and Active suspension. The numbers in parentheses indicate the initial number of eggs/larvae distributed in the tanks/chambers at each experimental stage. P-CH: probiotic chamber, C: control treatment, P: probiotic treatment. 1, 2 and 3: replicate tanks 1, 2 and 3.

\subsubsection{Axenic conditions}

To diminish the effects of water microbiota on early gut colonization, the larvae were initially reared under axenic conditions according to Situmorang et al., (2014). Briefly, two days post-fertilization eggs were washed from the mouth of an adult female Nile tilapia. Upon collection, the eggs were immersed in 30\% hydrogen peroxide (Merck-Millipore, Amsterdam, The Netherlands) and diluted in autoclaved synthetic freshwater (ASF) with a final active peroxide concentration of $2 \mathrm{~g} \mathrm{~L}^{-1}$ for $10 \mathrm{~min}$ at $26 \pm 1^{\circ} \mathrm{C}$. The synthetic freshwater contained $96 \mathrm{mg} \mathrm{L}^{-1} \mathrm{NaHCO}_{3^{\prime}} 60 \mathrm{mg} \mathrm{L}^{-1} \mathrm{CaSO}_{4} \cdot 2 \mathrm{H}_{2} \mathrm{O}, 60 \mathrm{mg} \mathrm{L}^{-1} \mathrm{MgSO}_{4}$ and 4 $\mathrm{mg} \mathrm{L}^{-1} \mathrm{KCl}$ in nanopure water (US Environmental Protection Agency, 2002). The eggs were subsequently washed four times with $250 \mathrm{~mL}$ of $0.2 \mu \mathrm{m}$-filtered ASF at $26 \pm 1^{\circ} \mathrm{C}$ to remove loose bacteria and damaged eggs. Twenty-four hours later, a second disinfection was conducted using $100 \mathrm{~mL}$ of $\mathrm{NaClO}(14 \%)$ in $1 \mathrm{~L}$ of ASF, following the same immersion protocol as described for day 1. During the immersion, the beakers were occasionally 
shaken to ensure the optimal penetration of disinfectant into the eggs. This disinfection method was applicable only prior to egg hatching, as this strategy was considered unsafe or lethal for larvae (Situmorang et al., 2014). On day 3, as soon as the eggs began to hatch, the disinfection process was conducted by the immersion of eggs/larvae into $1 \mathrm{~L}$ of ASF containing $100 \mathrm{mg} \mathrm{L}^{-1}$ Bronopol (2-bromo-2-nitropropane-1,3-diol; Sigma-Aldrich 13,4708, Zwijndrecht, The Netherlands) for $30 \mathrm{~min}$. Bronopol disinfection was performed daily from day 3 to 7 during the axenic stage. All disinfection procedures were performed in a laminar flow hood, and all equipment and tools were autoclaved prior to use.

Following disinfection on day 3 , the hatched eggs were aseptically distributed into 2-L sterile glass bottles (Duran GL45) containing $500 \mathrm{~mL}$ axenic incubation medium (Table S5.1) and incubated at a density of 300 eggs $\mathrm{L}^{-1}$. Air was provided to the bottles through a filter ( $0.25 \mu \mathrm{m}$, Whatman) from a single port safety cap (Duran DG), and the bottles were placed in a moving water-bath (Julabo SW23, $110 \mathrm{rpm}$ at $27^{\circ} \mathrm{C}$ ). During the axenic period (day 1 to 7 ), the larvae were not fed.

On day 8 , a total of 144 axenic larvae were equally distributed into the two probiotic chambers and exposed to the probiotic bacteria, whereas 120 larvae were equally distributed into the three AS aquaria and exposed to conventional (control) rearing conditions (see below for details).

\subsubsection{Probiotic conditions}

To rear the larvae during the probiotic stage a custom-made cabinet was used (Figure S5.1). The larvae were reared in two custom-made 2-L glass chambers installed inside the cabinet. The air in the cabinet was pressurized, and the internal surfaces were sterilized at hourly intervals through UV irradiation. The chambers were water-heated at $27 \pm 1^{\circ} \mathrm{C}$ using the space available within the double-layered outer glass wall. Air was supplied through $0.25-\mu \mathrm{m}$ syringe filters (Whatman) placed on single port safety caps (Duran DG). Larvae collection and water replacement were conducted through a bottom outlet valve (Figure S5.1). The larvae were sieved and washed with ASF daily and subsequently externally disinfected with Bronopol solution $\left(100 \mu \mathrm{L} \mathrm{L} \mathrm{L}^{-1}\right)$ for 30 min (see axenic stage disinfection from day 3 onwards). The incubation chambers were replaced daily with autoclaved chambers.

The probiotic strain, B. subtilis (Microbiologics 0269P, Mijdrecht, The Netherlands), was grown for $24 \mathrm{~h}$ on E-type agar containing $15 \mathrm{~g} \mathrm{~L}^{-1}$ bacteriological agar type $\mathrm{E}, 10 \mathrm{~g} \mathrm{~L}^{-1}$ Tryptone, $5 \mathrm{~g} \mathrm{~L}^{-1} \mathrm{NaCl}$, and $5 \mathrm{~g} \mathrm{~L}^{-1}$ yeast extract powder in $1 \mathrm{~L}$ of demineralized water. The bacterial colonies were further cultured in $100 \mathrm{~mL}$ of liquid medium containing $7 \mathrm{~g} \mathrm{~L}^{-1}$ $\mathrm{K}_{2} \mathrm{HPO}_{4^{\prime}} 2.0 \mathrm{~g} \mathrm{~L}^{-1} \mathrm{KH}_{2} \mathrm{PO}_{4^{\prime}} 1.0 \mathrm{~g} \mathrm{~L}^{-1}\left(\mathrm{NH}_{4}\right)_{2} \mathrm{SO}_{4^{\prime}} 1.0 \mathrm{~g} \mathrm{~L}^{-1}$ glucose, $0.5 \mathrm{~g} \mathrm{~L}^{-1}$ sodium citrate, and $0.1 \mathrm{~g} \mathrm{~L}^{-1} \mathrm{MgSO}_{4} \cdot 7 \mathrm{H}_{2} \mathrm{O}$ in demineralized water for another $24 \mathrm{~h}$. Prior to use, the cultures were centrifuged for $7 \mathrm{~min}(8000 \times \mathrm{g})$ to pellet the B. subtilis. The supernatant was 
discarded, and the cell density was adjusted to $1 \times 10^{7} \mathrm{cfu} \mathrm{mL}^{-1}$ with $1.5 \mathrm{~L}$ ASF water as spectrophotometrically determined by optical density (OD ${ }_{600}$ ) (Bio-Rad SmartSpec 3000). In the probiotic chambers, the water containing B. subtilis was replaced daily.

\subsubsection{Active suspension tanks}

Six 20-L aquaria, each connected to a separate 120-L active suspension tank (AST), were used for rearing the larvae under conventional conditions. One month prior to the experiment, 10 adult tilapias were stocked per AST to initiate microbial growth. Prior to the addition of the axenic larvae, adult tilapias were removed, and the water from all six ASTs was mixed and re-distributed over the tanks. Water and suspended solids in the ASTs were constantly mixed and aerated, while the temperature was maintained at $27^{\circ} \mathrm{C}$. Total ammonia nitrogen (TAN-N), nitrite $\left(\mathrm{NO}_{2}^{-}-\mathrm{N}\right)$, nitrate $\left(\mathrm{NO}_{3}^{-}-\mathrm{N}\right)$, dissolved oxygen (DO), $\mathrm{pH}$ and temperature $\left({ }^{\circ} \mathrm{C}\right)$ were monitored daily in each AST.

During their first week in the ASTs (day 8 to 14), the larvae from the control treatment and in the probiotic chambers were not fed (Figure 5.1). On day 14, the larvae from both probiotic chambers were mixed and redistributed over the three active suspension tanks (40 larvae/tank). Thereafter, the larvae from both treatments were fed daily to apparent satiation (30 $\mathrm{min}$ ) at 09:00, 12:30 and 16:00 with a commercial crumble larval feed (Skretting Gemma Wean M0.5, 300-500 $\mu \mathrm{m}, 58 \%$ crude protein, 17\% crude fat, $10 \%$ ash, $0.6 \%$ fibre and $1.3 \%$ phosphorus). The feed was divided into daily portions of $4 \mathrm{~g}$ in 15 $\mathrm{mL}$ Falcon tubes and subsequently sterilized with cobalt-60 gamma irradiation at $25 \mathrm{kGy}$ (Synergy Health, Ede, The Netherlands) prior to the experiment to minimize the viable microbial load entering with the feed, and the $16 \mathrm{~S}$ ribosomal RNA (rRNA) gene-targeted PCR using DNA extracted from irradiated feed did not yield any products.

\subsubsection{Verification of axenic and probiotic conditions by cultivation}

During the axenic and probiotic stages, daily samples of the culture medium, eggs and media/water were monitored for the presence of viable bacterial cells. The eggs, larvae and $1 \mathrm{~mL}$ of water were separately added into $10 \mathrm{~mL}$ of liquid medium (as previously described for B. subtilis) and incubated at $37^{\circ} \mathrm{C}$ for $24 \mathrm{~h}$. The next day, the samples were streaked onto 2YT agar (Biotrading K604P090KP, Mijdrecht, The Netherlands) and incubated for $24 \mathrm{~h}$ at $37^{\circ} \mathrm{C}$. At $48 \mathrm{~h}$ after inoculation, the agar plates were visually assessed for microbial growth.

\subsubsection{Sampling of gut and water for bacterial community profiling}

For each of the aquaria/chambers, the gut samples from four larvae were collected on days 14, 21 and 28 (Table S5.2, sample meta-data), and the water was also sampled from each aquarium/chamber after filtering $1 \mathrm{~L}$ of water through 0.45 - and $0.2-\mu \mathrm{m}$ membrane 
filters (Millipore HAWP-04700 and Millipore GTTP-04700). All samples were frozen in liquid nitrogen and stored at $-80^{\circ} \mathrm{C}$ until further analysis. All gut and water samples were stored and individually analysed. The detailed protocols on gut and water sampling are described in Giatsis et al. (2014).

\subsubsection{Bacterial community profiling}

DNA was extracted from gut samples using the DNeasy Blood \& Tissue Kit (Qiagen, Venlo, The Netherlands) according to the manufacturer's protocol, with some modifications (Giatsis et al., 2014). For DNA extraction from the water samples, the FastDNA SPIN kit for soil (MP Biomedicals, Ohio, USA) was used. The DNA concentrations were measured with a NanoDrop ND-1000 spectrophotometer (NanoDrop ${ }^{\circledR}$ Technologies, Wilmington, DE), and the DNA samples were stored at $-80^{\circ} \mathrm{C}$ until further use. Detailed protocols on gut and water DNA extraction are described elsewhere (Giatsis et al., 2014).

For 16S rRNA gene-based microbial composition profiling, barcoded amplicons from the V4 region of $16 \mathrm{~S}$ rRNA genes were generated by PCR using the $515 \mathrm{~F}$ and $806 \mathrm{R}$ primers (Caporaso et al., 2010). Seventy different barcodes were used per library, in which the forward and reverse primer of one sample always carried the same barcode. The primer sequence and barcode were separated by a 2-nucleotide linker sequence (GA for 515F and CG for 806R). The extracted DNA was diluted to a concentration of $20 \mathrm{ng} \mathrm{\mu L}^{-1}$ based on NanoDrop (NanoDrop Technologies, Wilmington, DE) spectrophotometric readings. The PCR conditions, DNA purification and library preparations were performed according to Giatsis et al. (2015). The nucleotide sequences were generated using an Illumina HiSeq 2000 sequencer at GATC-Biotech, Konstanz, Germany. Raw sequence data were deposited into the Sequence Read Archive (SRA) at the NCBI under accession number SRP062681.

NG-Tax, an in-house pipeline, was used for the analysis of the 16S rRNA gene sequencing data (Ramiro-Garcia et al., 2016). Briefly, paired-end libraries were filtered to contain only read pairs with perfectly matching barcodes, and these barcodes were used to separate reads according to sample. The operational taxonomic units (OTUs) were assigned and classified using an open reference approach and a customized SILVA 16S rRNA gene reference database (Quast et al., 2013).

\subsubsection{Data handling and statistical analysis}

The Bray Curtis dissimilarity was calculated based on square root-transformed relative abundance data. Principal coordinate analysis (PCoA) was performed to represent the samples in a low dimensional space; thus, the relative distances of all points represent the relative dissimilarities of the samples according to the Bray Curtis index. All statistical analyses were performed using the multivariate statistical software package Primer V7 
(Primer-E Ltd, Plymouth, UK). BLAST searches were used to identify the closest relatives of selected OTUs (members of the genus Bacillus) (Altschul, 1997). Multiple sequence alignments of the sequences were performed in ClustalW2-Phylogeny using neighbourjoining as the clustering method, and the corresponding Newick tree file was visualized using a phylogram constructed in Treedyn (Chevenet et al., 2006).

\subsection{Results}

\subsubsection{Axenic stage}

In total, 380 fertilized eggs were available at the start of the experiment. By the end of the hatching period, 304 larvae (80\% of all eggs) successfully hatched. On day 7, 120 of the axenic larvae were equally distributed into active suspension tanks C1, C2 and C3 (named as "control treatment"), whereas the remaining larvae were exposed to a high load of $B$. subtilis in chambers $\mathrm{P}-\mathrm{CH} 1$ and $\mathrm{P}-\mathrm{CH} 2$ (named "probiotic treatment") for one week.

Medium and egg samples cultured on agar plates showed no proliferation of microbes throughout the axenic period, and 16S rRNA gene-targeted PCR using DNA extracted from washed and antibiotic-treated eggs and larvae yielded no products, confirming the axenic conditions.

\subsubsection{Probiotic stage}

The $16 \mathrm{~S}$ rRNA profiling of water microbiota from chambers $\mathrm{P}-\mathrm{CH} 1$ and $\mathrm{P}-\mathrm{CH} 2$ on day 14 confirmed the presence of the probiotic strain in both chambers albeit at different relative abundances. P-CH2 was dominated with Bacillus, whereas $\mathrm{P}-\mathrm{CH} 1$ was dominated with Pseudomonas (Figure 5.2). In both chambers, several OTUs belonging to the genus Bacillus were present; however, the most abundant Bacillus OTU (OTU 814) had 100\% sequence identity with the added probiotic strain of B. subtilis (Figure S5.2). Pseudomonas OTU 338 was present in the water of both chambers at a relative abundance of $>20 \%$.

Subsequent analyses of the gut samples from larvae raised in the probiotic chambers showed that $B$. subtilis was among the most dominant species, regardless of the observed differences in the relative abundance of $B$. subtilis in the corresponding water samples. At the end of the probiotic treatment (day 14), B. subtilis accounted for approximately half of all bacteria in the gut (average relative abundance). A comparison between the gut samples from control ( $\mathrm{C} 1, \mathrm{C} 2$ and $\mathrm{C} 3$ tanks) and probiotic treatments ( $\mathrm{P}-\mathrm{CH} 1$ and $\mathrm{P}-\mathrm{CH} 2$ chambers) indicated a clear difference in the composition of the gut microbiota (Figure 5.3a). This difference reflected, in part, the high relative abundance of $B$. subtilis in the gut of larvae from the probiotic treatment (and the absence of these bacteria from the 
control), according to the SIMPER analysis results (contribution: 25\%). Other discriminant OTUs were members of the genera Nocardia, Mycobacterium, Rhodococcus, Rhodanobacter and Halomonas (Table S5.3).

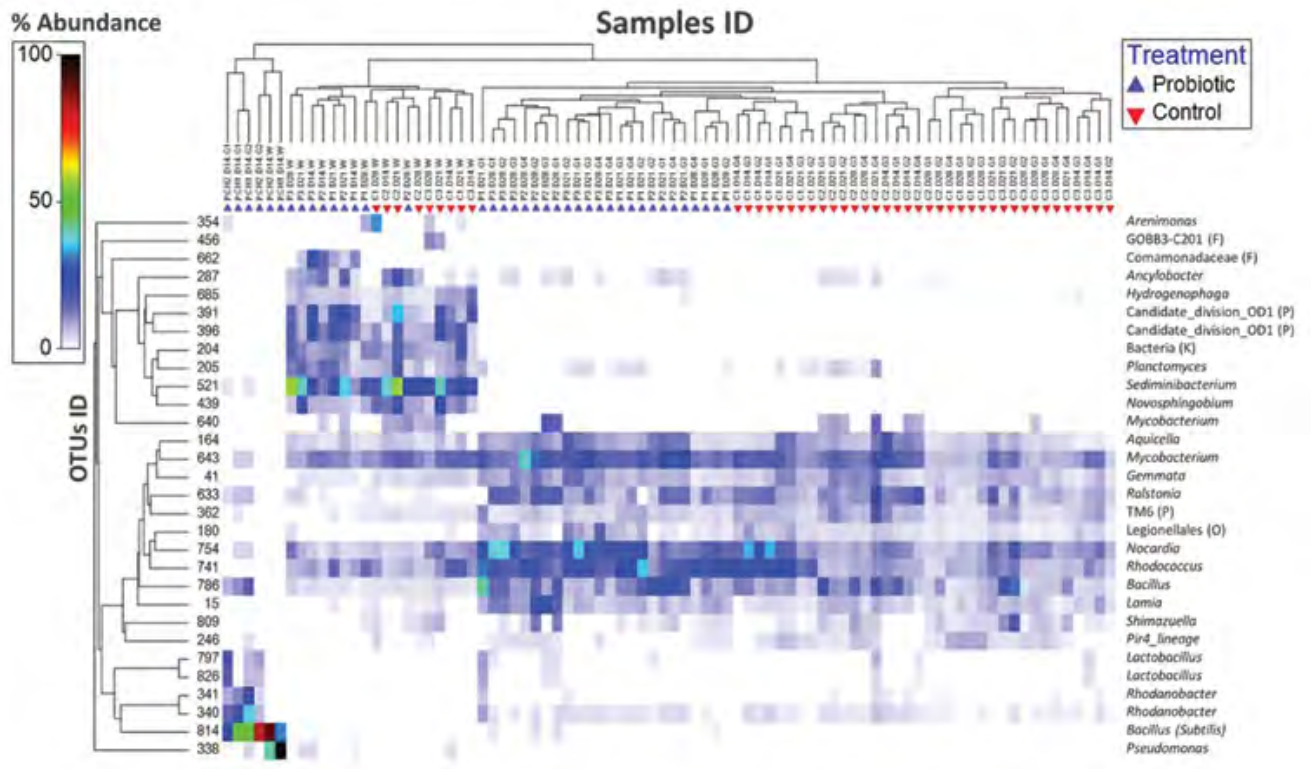

Figure 5.2. Heatmap of the 30 most predominant OTUs among all water and gut samples. Each column in the heatmap represents a sample, and each row represents an OTU. The OTUs were clustered based on group average, as groups of OTUs better define sample clusters. Only the first thirty OTUs contributing most to these clusters are displayed. The samples were clustered according to the unweighted pair group method with arithmetic mean (UPGMA) hierarchical clustering on the basis of Bray Curtis dissimilarity and based on the complete OTU dataset. The colours are proportional to the increasing percent relative OTU abundance (from white: lower, to red: higher) within each sample. P1-3 and C1-3: Replicate active suspension tanks 1-3 of the probiotic and control treatment, respectively. D14, 21 and 28: Experimental days 14, 21 and 28. G1-4: The number of replicate gut samples from each tank. G and W: Gut and water samples, respectively. P-CH1 and 2 (probiotic chamber 1 and 2) indicate that water samples were obtained from the probiotic chamber at the end of probiotic period (day 14). Taxonomy (right column) indicates the genus of each OTU ID (left column) unless otherwise stated (i.e., order (O), family (F) and phylum (P)). 

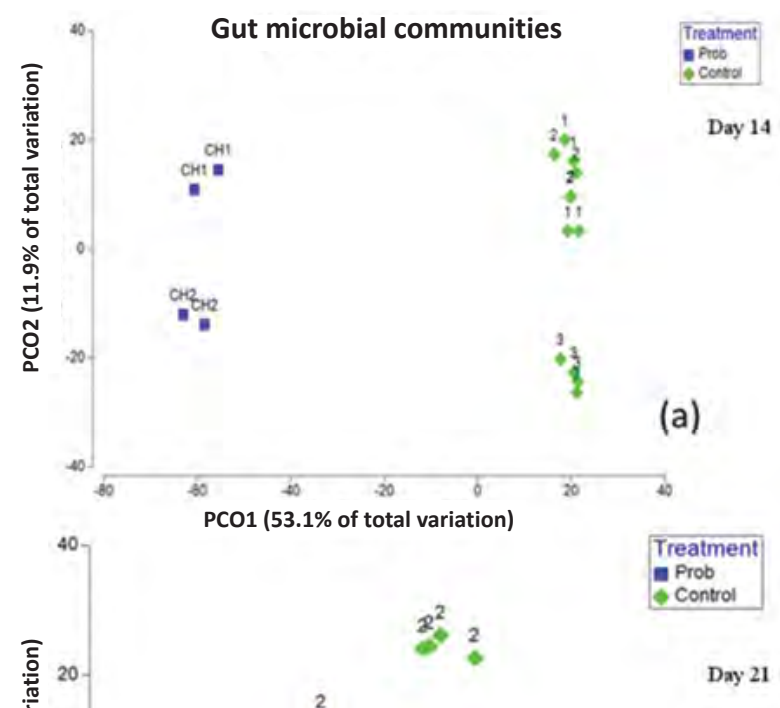

(a)

Day 14

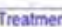

Treatment

- Costal

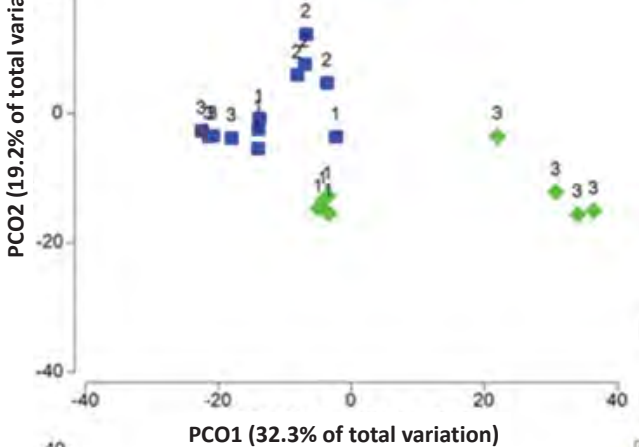

(b)

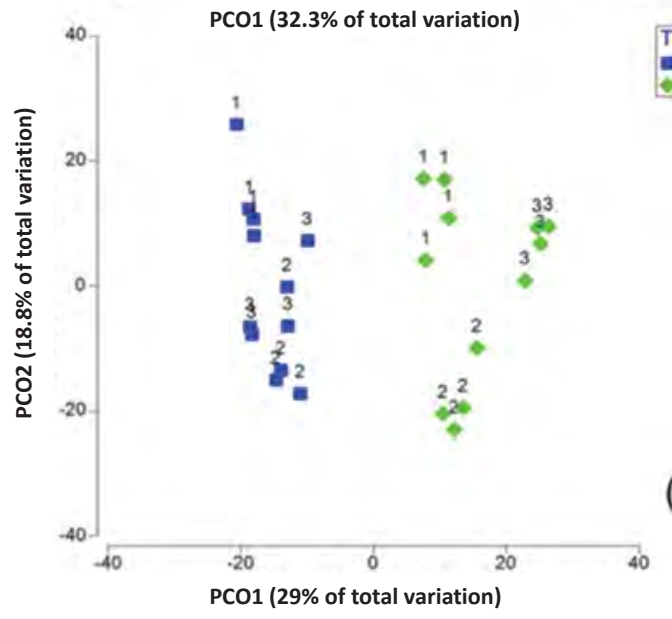

Day 28

(c)

Figure 5.3. Principal coordinate analysis ( $P C O A)$ of the gut microbial communities based on the square root-transformed relative abundance data of OTUs. The relative distances of all points represent the relative dissimilarities of the samples according to the Bray Curtis index. Plots ( $a, b$ and $c)$ : ordinations of all gut samples from probiotic and control treatment from days 14, 21 and 28, respectively. The percentage of total variation explained by each PCo axis is shown in the parentheses. 


\subsubsection{Active suspension stage}

At the end of the probiotic stage (day 14), larvae from the probiotic chambers were transferred to active suspension tanks (P1, P2 and P3). One week after exposure to conventional rearing conditions (day 21), their gut microbiota was significantly different from that of the control treatment larvae, which had been acclimated to non-sterile conditions for one week longer (Figure 5.3b and Table S5.4). The replicate aquaria of the control treatment were significantly more dispersed than those of the probiotic treatment according to multivariate permutation dispersion $\left(P_{\text {perm }}: 0.011\right)$ (Table S5.5). However, the observed lower dispersion within the probiotic treatment no longer reflected the presence of $B$. subtilis in the gut, as the relative abundance of these bacteria at day 21 was below detection level. The most predominant OTUs in the probiotic treatment were members of the genera Bacillus, Rhodococcus, Nocardia, Mycobacterium, Ralstonia and Aquicella (Figure 5.2). These taxa were also among the most predominant bacteria observed in the gut of the control treatment on day 21 but at different relative abundances. One Bacillusaffiliated OTU (OTU 786) was present in all gut samples of both treatments on day 21, with an average relative abundance of $4.1 \%(S D \pm 2.3)$. BLAST analysis showed that this bacterium was a different species than the administered strain, as its 16S rRNA gene sequence was only $94 \%$ identical to that of the probiotic strain of B. subtilis (Figure S5.2).

A comparison of the gut microbiota on day 28 showed significantly different communities in the two treatments (Figure 5.3c and Table S5.4). As on day 21, the probiotic strain remained below the detection level. The most predominant genera at day 28 in both treatments were similar to those observed at day 21 , albeit at different relative abundances. The within-treatment variability of replicate tanks was no longer significantly different $\left(P_{\text {perm }}: 0.121\right)$ between control and probiotic treatments (Table S5.5). Notably, the homogeneity of dispersion is a precondition to accurately interpret, but not perform, a PERMANOVA. On day 21, significant differences in the gut communities were detected in both PERMDISP (dispersion) and PERMANOVA (location). Thus, to uncover the nature of the differences among groups, the results are also discussed with respect to the average within- and between-group dissimilarities and the position of the samples from different groups in the PCOA.

\subsubsection{Succession of water and gut microbiota}

The effect of time on the water and gut microbiota composition was evaluated by comparing the profiles on days 14, 21 and 28. The water microbiota in the tanks was not significantly different between the two treatments on any of the sampling days, but a different pattern was revealed over time (Table S5.6), i.e., water microbial communities from the control treatment clearly clustered by tank, whereas this pattern was not observed in the probiotic treatment (Figure 5.4a and b). Sediminibacterium (OTU 521) was 
the most abundant genus in the water of both treatments on days 21 and 28, with average relative abundances between $18 \%$ and $35 \%$, respectively (Figure 5.2).

Regarding the gut samples, the cluster analysis revealed clearly different patterns between treatments. In the control treatment, the variability of the gut microbial communities was higher between tanks than between days (Figure 5.4c), consistent with the pattern observed in the water (Figure 5.4a). In the probiotic treatment, the tank effect was not as clear as in the case of the control (Figure 5.4d).

\section{Water microbial communities}
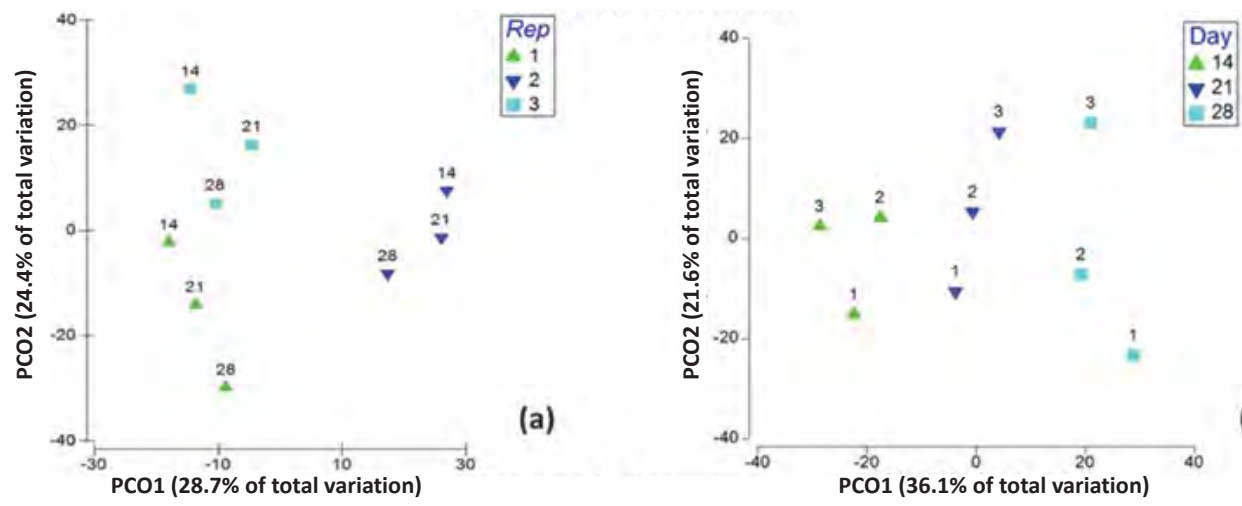

(b)
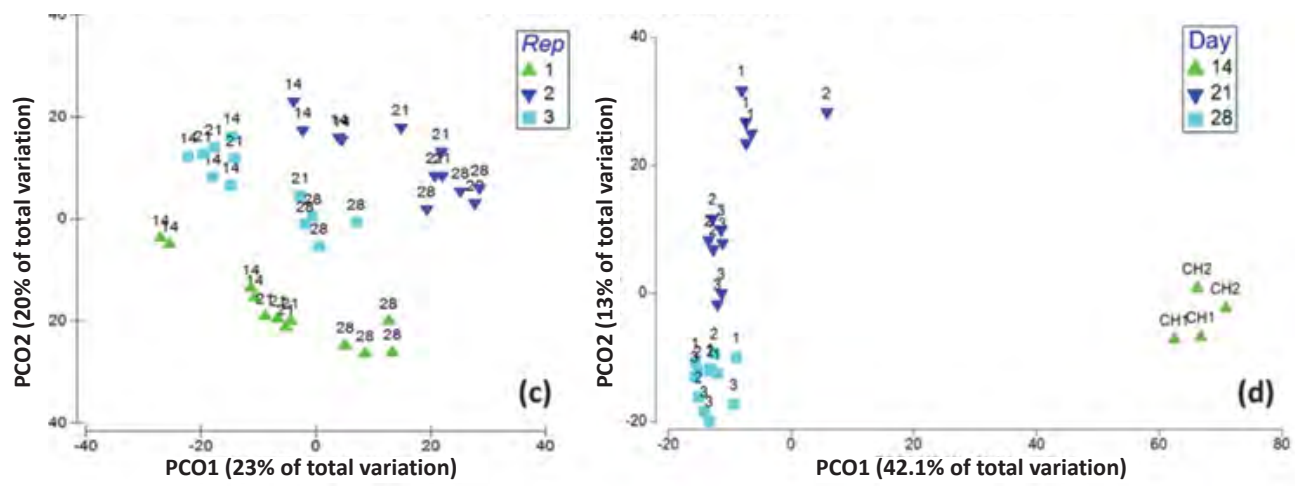

Figure 5.4. Principal coordinate analysis ( $\mathrm{PCOA}$ ) of microbial communities in the gut and water samples of the control and probiotic treatments. The plots are based on the square root-transformed relative abundance data for the OTUs. The relative distances of all points represent the relative dissimilarities of the samples according to the Bray Curtis index. Ordination plots of water ( $a$ and $b$ ) and gut ( $c$ and $d$ ) microbial communities from the control ( $a$ and $c$ ) and probiotic treatments ( $b$ and d), respectively. The numbers 14,21 and 28 indicate the three experimental days that the samples were collected. Rep 1-3: replicate tank 1 to 3 . The percentage of total variation explained by each PCo axis is shown in the parentheses. 


\subsection{Discussion}

Probiotics have been widely applied in aquaculture for many years. However, assessing the effectiveness of their use is problematic because the probiotic strains residing in the gut transiently and rapidly fall below detection limits. This effect most likely reflects the low survival and proliferation rate of probiotics in the fish gut (Gatesoupe, 1999; Grześkowiak et al., 2012). For prolonged gut colonization by probiotics, it is paramount to understand the principles governing microbial community assembly and the persistence of specific populations.

In the present study, we attempted to enhance the colonization success of the probiotics in a "virgin" gut ecosystem by maintaining the larvae in axenic conditions prior to exposure to a probiotic strain. The results indicated that $B$. subtilis was present in the waters of both probiotic chambers, albeit at different relative abundances. In the first chamber, $B$. subtilis was the most dominant water OTU; in the second chamber, populations belonging to the genus Pseudomonas were present at a higher relative abundance, although the same amount of $B$. subtilis was added daily to both chambers. Apparently, we were not successful in maintaining the gnotobiotic conditions of the water, as both chambers were contaminated with Pseudomonas.

However, regardless of the presence of Pseudomonasinthe water ofboth P-CH,Pseudomonas was barely detected in the gut at that time point. These results demonstrated that (a) $B$. subtilis can be successfully transferred to the gut through water, and (b) Pseudomonas cannot be successfully transferred to the gut irrespective of its abundance in the source water. Members of the genus Pseudomonas are non-sporulating, aerobic Gram-negative rods observed in a wide range of terrestrial and aquatic environments in addition to plant and animal-associated ecosystems. Consistent with this broad environmental distribution, these bacteria exhibit metabolic versatility (Pérez et al., 2010; Özen and Ussery, 2012). In fish, Pseudomonas is commonly observed in the faeces and gut of both salt and fresh water species (Austin, 2006; Cahill, 1990). Various phenomena, such as the competitive exclusion of Pseudomonas by $B$. subtilis, differences in the ecological preference/adaptability of the two species in the gut and host selectivity for Bacillus but not Pseudomonas, may have played a role in the recovery of $B$. subtilis but not Pseudomonas from the guts of tilapia larvae during probiotic treatment.

In the present study, B. subtilis was only transiently detected and thus was not included in the stable larval microbiota. One week after the larvae were exposed to conventional aquaculture conditions (day 21 ), the abundance of $B$. subtilis was already below detection level. This finding underscores the challenge of successfully colonizing the fish gut with a probiotic strain. The presence of this strain in the gut can be expected only until a few days after probiotic discontinuation, consistent with previous studies reporting that probiotic strains added through water or feed could be detected in the guts of fish and shrimp 
for only a few days after discontinuing the probiotic application (Kim and Austin, 2006; Vaseeharan and Ramasamy, 2003; Sharifuzzaman et al., 2014; Zhang et al., 2009; Robertson et al., 2000). In a recent study, Standen et al. (2015) observed that the presence of a multispecies probiotic containing Lactobacillus reuteri, Bacillus subtilis, Enterococcus faecium and Pediococcus acidilactici gradually declined after probiotic cessation. However, the detection of each probiotic strain in the gut varied between 6 and 18 days after reverting to the control diet, suggesting that the persistence of probiotics in the gut is speciesspecific. Furthermore, the probiotic supplemented feed was administered to adult tilapia for eight weeks, whereas here, $B$. subtilis was administered to axenic tilapia larvae for one week. The dosage and duration of supplementation and the selection of the probiotic strain/s might influence colonization success, and the persistence of the probiotic might also depend on the developmental state of the animal (Pérez et al., 2010; Ramos et al., 2013; Gerritsen et al., 2011).

In the present study, the development of gut microbial communities in the two treatments revealed different patterns. The gut microbiota in the control treatment were more affected by spatial (tank) rather than temporal differences (time), i.e., the samples clustered according to replicate tank rather than sampling day (Figure 5.4c). Interestingly, the spatiotemporal patterns observed in gut bacterial communities were also observed in the water microbiota of the control treatment. In a previous study on tilapia larvae, we observed that tank replication determined the inter-individual variation of gut microbiota (Giatsis et al. 2014). Here, this finding was differently applied for each treatment. This difference could be associated with the initial contact of larvae with the probiotic strain. At the time larvae from the probiotic treatment were introduced to conventional conditions, their guts were already colonized with certain bacteria (primarily B. subtilis), whereas the larvae from the control treatment were introduced to conventional conditions while their guts were germ-free.

The successful transfer via water and the high relative abundance of the probiotic strain in the gut indicate that it is conceivable to inoculate the gut community with bacteria during early gut development. At the end of the probiotic stage, four gut samples were collected from both probiotic chambers. The observed inter-individual variation in the abundance of the probiotic strain in the gut suggests that the results should be interpreted with caution. Studies on the use of probiotics in humans and animals have also reported high inter-individual variation, even for identically treated groups (Garcia-Mazcorro et al., 2011; Baxter et al., 2015; Montesi et al., 2005; Ling et al., 2013). The abundance of a probiotic strain in the gut or faeces is neither clear-cut proof of successful probiotic use nor evidence of probiosis, primarily reflecting the difficulty in establishing the precise relationships between the health benefits and the presence and/or relative abundance of a specific microbe (except for specific pathogens) (Gerritsen et al., 2011). Notably, inter-individual variation could certainly mask treatment effects by either type-I or type-II errors. Thus, 
more data points (higher statistical power) should be included in future studies to verify whether the observed correlations are maintained.

The observed low persistence of the probiotic strain in the gut could indicate a lack of ecological preference or adaptability of the probiotic strain in the gut and/or host selectivity against the probiotic. Nevertheless, the gut communities remained different between treatments, even after discontinuation of the probiotic and despite receiving the same diet and living in water containing similar microbial profiles (Figure 5.5).

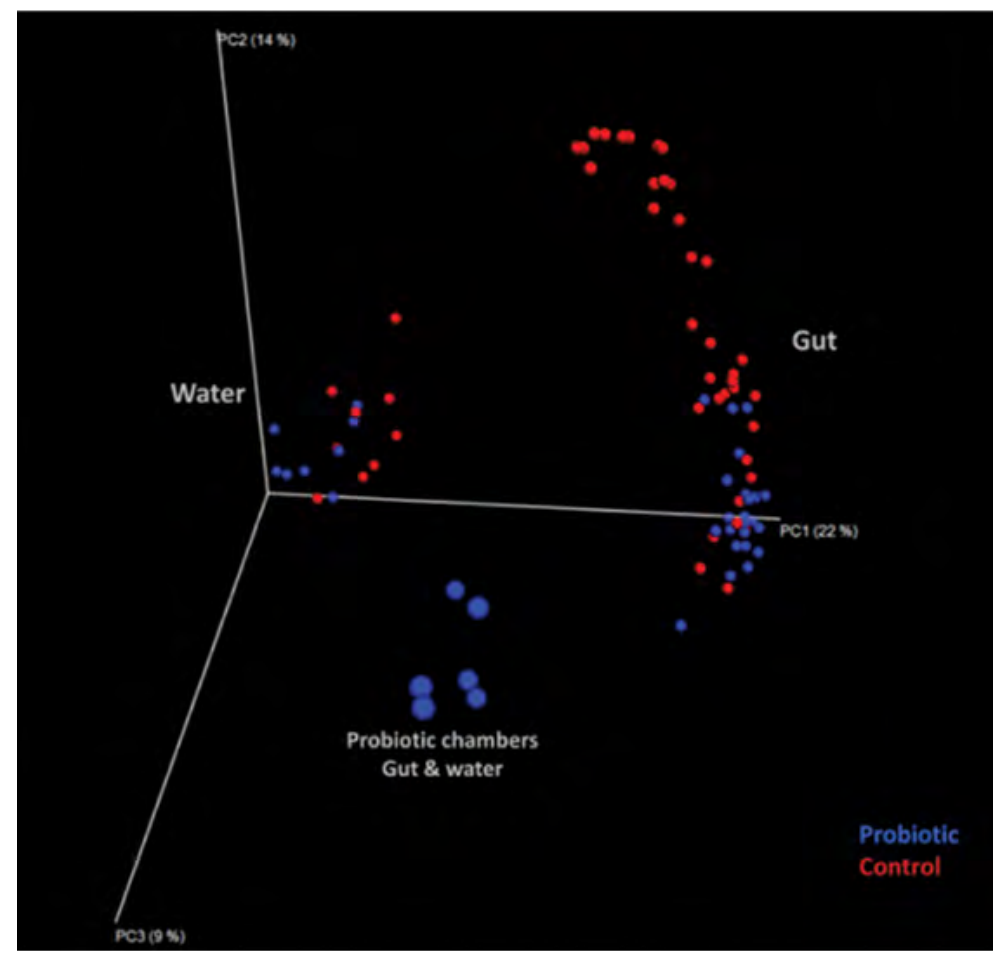

Figure 5.5. Principal coordinate analysis ( $P C O A)$ of the gut (right) and water (left) microbial communities from all sampling days (14, 21 and 28) and treatments (probiotic and control). The blue spheres along the PC3 (centre) correspond to water and gut samples from the probiotic chambers on day 14. This analysis was based on the square root-transformed relative abundance data of the OTUs. The relative distances of all points represent the relative dissimilarities of the samples according to the Bray Curtis index. The percentage of total variation explained by each of the three first PCo axes is shown in the parentheses.

It is doubtful (although there is no clear-cut evidence) that the presence of Pseudomonas in the probiotic chambers induced the observed probiotic legacy effects, as Pseudomonas, although present in the water, was nearly absent from the larval gut. It is more likely that the initial presence of $B$. subtilis led to a different sequence of events of bacterial colonization, reflecting the synergistic or antagonistic interactions between the bacteria already present and/or other bacteria entering the gut. It is also likely that the transition from axenic to either conventional or probiotic conditions differentially modulated the 
immune response and mucosal innate immunity of the larvae. The responses of $\lg A$, cytokine production and the development of mucosal T-regulatory cells were likely reduced in germ-free animals through the activation of TLR-dependent pathways (Gomez de Agüero et al., 2016; Fagundes et al., 2012; Kubinak and Round, 2012). TLR9 was expressed on the colonic apical surface in wild type but not germ-free mice (Ewaschuk et al., 2007). These results demonstrated that the gut microbiota alters the way the host reacts to infectious stimuli or particular bacterial taxa (Neish, 2014) entering the gut, and this difference could also be the case in the present study. Differences in the initial priming of the immune system in the probiotic group are certainly among the potential mechanisms (Thompson et al., 2010; Corthésy et al., 2007).

After the discontinuation of probiotic administration, differences in the gut microbiota between treatments primarily reflected differences in the relative abundance of the genera Nocardia, Mycobacterium, Rhodococcus, Rhodanobacter, Halomonas and Ralstonia. Most of these genera have been identified in previous studies on tilapia larvae and other fish species. The genus Rhodococcus has been reported in the guts of tilapia, sole, red rock fish, Norwegian mackerel, USA smelt, rainbow trout and shrimp (Giatsis et al., 2015; Kim et al., 2007; Sanchez et al., 2012; Spanggaard et al., 2000; Huber et al., 2004), and the genus Nocardia has been reported in the guts of tilapia (Kohl et al., 2014; Giatsis et al., 2015) and Atlantic salmon (Bakke-McKellep et al., 2007). Ralstonia has been observed in the guts of seabass (Carda-Diéguez et al., 2014), rainbow trout (Kim et al., 2007; Navarrete et al., 2012; Pond et al., 2006), yellow catfish (Wu et al., 2010), zebrafish (Rawls et al., 2004) and shrimp (Durand et al., 2010). Furthermore, members of the genus Halomonas have been reported in the guts of Arctic charr (Ringo et al., 2006), Atlantic cod (Fjellheim et al., 2011), Midas cichlids (Franchini et al., 2014), queen conch (Carrascal et al., 2014) and Artemia brine shrimp (Tkavc et al., 2011; Riddle et al., 2013). These findings indicate that some of the predominant genera observed in the present study could represent common members of the gut microbiota of tilapia larvae or fish in general, suggesting that (a) host-specificity for particular microbial taxa is modulated by selective pressures within the host gut, and (b) these taxa are involved in major metabolic functions in the fish gut. Host-selective capabilities have been revealed in axenic zebrafish (Danio rerio) that received a faecal transplant derived from mice. The implanted mouse community subsequently shifted towards a state resembling a native zebrafish community (Rawls et al., 2006). In addition, zebrafish originating from the wild shared a core gut microbiota with those reared in captivity, demonstrating a host-specific microbial community in the gut (Roeselers et al., 2011). The observed differences in the abundance of these genera within and between studies could reflect a certain degree of influence of the environmental microbiota (i.e., available bacteria, including the probiotic strain used in the present study), communitylevel interactions and dietary interventions, underlying powerful organizing principles in community composition. 
To what extent post-treatment gut microbial uniformity or distinctness reflects a sustained effect of the probiotic remains unknown. We suggest that future studies focus on the long-term effects of probiotic legacy during the early developmental stages of animals. To observe a general phenomenon, future experiments are needed to determine how this effect compares with that of antibiotic or dietary interventions. It has been suggested that legacy effects in humans play a role in defining the microbial structure during early life stages, and these effects can be minimized based on the diet of the host (Turnbaugh et al., 2009). If this idea also applies to animals, then the early administration of a probiotic strain, accompanied by continuous prebiotic administration, could further extend probiotic residence in the gut, even after its discontinuation.

\section{Acknowledgements}

The authors thank M.P. Rizqi, I. Tankowski, M. Ter Veld and H. Heilig for assistance with fish husbandry, sample collection and laboratory analysis. This work was funded by The European Community's Seventh Framework Programme (FP7/2007-2013) under grant agreement no. 227197 Promicrobe "Microbes as positive actors for more sustainable aquaculture". 


\section{Supplementary material}

Table S5.1. Antibiotics and antifungals used for the preparation of the incubation medium. All components were diluted in autoclaved synthetic freshwater (ASF) to the concentration indicated in the table. Original protocol has been obtained by Situmorang et al. (2014) and modifications have been applied regarding the addition of Gentamycin in the incubation medium.

\begin{tabular}{lllll}
\hline Name & Concentration & Dosage & Description & Source \\
\hline Kanamycin Sulphate & Sigma T7783 & $1 \mathrm{~mL} \mathrm{~L}^{-1}$ & Broad spectrum antibiotic & (Situmorang et al., 2014) \\
Rifampicin & Sigma K1377 & $1 \mathrm{~mL} \mathrm{~L}^{-1}$ & Broad spectrum antibiotic & (Situmorang et al., 2014) \\
Ampicillin sodium salt & Sigma A0166 & $1 \mathrm{~mL} \mathrm{~L}^{-1}$ & Broad spectrum antibiotic & (Situmorang et al., 2014) \\
Trimethoprim & Sigma 89307 & $1 \mathrm{~mL} \mathrm{~L}^{-1}$ & Bacteriostatic antibiotic & (Situmorang et al., 2014) \\
Gentamicin & Sigma G1397 & $50 \mu \mathrm{L} \mathrm{L}^{-1}$ & Gram-negative antibiotic & Rosco NeoSensitabs \\
Amphotericin B & Sigma A9528 & $100 \mathrm{LL} \mathrm{L}^{-1}$ & Polyene antifungal & (Situmorang et al., 2014) \\
Fluorescent Brightener 28 & Sigma F3543 & $2.5 \mathrm{~mL} \mathrm{~L}^{-1}$ & Antifungal & (Situmorang et al., 2014) \\
\hline
\end{tabular}

Table S5.2. Meta-data of gut $(G)$ and water $(W)$ samples from the control $(C)$ and probiotic $(P)$ treatment. Microbial communities were characterized on day (D) 14, 21 \& 28 in the probiotic chamber (P-CH) and/or the active suspension tanks. Two replicate probiotic chambers and three replicate active suspension tanks were sampled per treatment (C1-3 \& P1-3) from which a water and four replicate gut samples (G1-4) were analysed.

\begin{tabular}{|c|c|c|c|c|c|c|c|}
\hline No & Sample_ID & Treatment & Culture system & Biome & Day & Tank & Replicate \\
\hline 1 & P-CH1.D14.W & Probiotic & Probiotic chamber & Water & 14 & 1 & - \\
\hline 2 & P-CH2.D14.W & Probiotic & Probiotic chamber & Water & 14 & 2 & - \\
\hline 3 & C1.D14.W & Control & Active suspension & Water & 14 & 1 & - \\
\hline 4 & C2.D14.W & Control & Active suspension & Water & 14 & 2 & - \\
\hline 5 & C3.D14.W & Control & Active suspension & Water & 14 & 3 & - \\
\hline 6 & P1.D14.W & Probiotic & Active suspension & Water & 14 & 1 & - \\
\hline 7 & P2.D14.W & Probiotic & Active suspension & Water & 14 & 2 & - \\
\hline 8 & P3.D14.W & Probiotic & Active suspension & Water & 14 & 3 & - \\
\hline 10 & C1.D21.W & Control & Active suspension & Water & 21 & 1 & - \\
\hline 11 & C2.D21.W & Control & Active suspension & Water & 21 & 2 & - \\
\hline 12 & C3.D21.W & Control & Active suspension & Water & 21 & 3 & - \\
\hline 13 & P1.D21.W & Probiotic & Active suspension & Water & 21 & 1 & - \\
\hline 14 & P2.D21.W & Probiotic & Active suspension & Water & 21 & 2 & - \\
\hline 15 & P3.D21.W & Probiotic & Active suspension & Water & 21 & 3 & - \\
\hline 17 & C1.D28.W & Control & Active suspension & Water & 28 & 1 & - \\
\hline 18 & C2.D28.W & Control & Active suspension & Water & 28 & 2 & - \\
\hline 19 & C3.D28.W & Control & Active suspension & Water & 28 & 3 & - \\
\hline 20 & P1.D28.W & Probiotic & Active suspension & Water & 28 & 1 & - \\
\hline 21 & P2.D28.W & Probiotic & Active suspension & Water & 28 & 2 & - \\
\hline 22 & P3.D28.W & Probiotic & Active suspension & Water & 28 & 3 & - \\
\hline 24 & C1.D14.G1 & Control & Active suspension & Whole gut & 14 & 1 & 1 \\
\hline 25 & C1.D14.G2 & Control & Active suspension & Whole gut & 14 & 1 & 2 \\
\hline 26 & C1.D14.G3 & Control & Active suspension & Whole gut & 14 & 1 & 3 \\
\hline 27 & C1.D14.G4 & Control & Active suspension & Whole gut & 14 & 1 & 4 \\
\hline 28 & C2.D14.G1 & Control & Active suspension & Whole gut & 14 & 2 & 1 \\
\hline 29 & C2.D14.G2 & Control & Active suspension & Whole gut & 14 & 2 & 2 \\
\hline 30 & C2.D14.G3 & Control & Active suspension & Whole gut & 14 & 2 & 3 \\
\hline 31 & C2.D14.G4 & Control & Active suspension & Whole gut & 14 & 2 & 4 \\
\hline 32 & C3.D14.G1 & Control & Active suspension & Whole gut & 14 & 3 & 1 \\
\hline
\end{tabular}




\begin{tabular}{|c|c|c|c|c|c|c|c|}
\hline No & Sample_ID & Treatment & Culture system & Biome & Day & Tank & Replicate \\
\hline 33 & C3.D14.G2 & Control & Active suspension & Whole gut & 14 & 3 & 2 \\
\hline 34 & C3.D14.G3 & Control & Active suspension & Whole gut & 14 & 3 & 3 \\
\hline 35 & C3.D14.G4 & Control & Active suspension & Whole gut & 14 & 3 & 4 \\
\hline 36 & C1.D21.G1 & Control & Active suspension & Whole gut & 21 & 1 & 1 \\
\hline 37 & C1.D21.G2 & Control & Active suspension & Whole gut & 21 & 1 & 2 \\
\hline 38 & C1.D21.G3 & Control & Active suspension & Whole gut & 21 & 1 & 3 \\
\hline 39 & C1.D21.G4 & Control & Active suspension & Whole gut & 21 & 1 & 4 \\
\hline 40 & C2.D21.G1 & Control & Active suspension & Whole gut & 21 & 2 & 1 \\
\hline 41 & C2.D21.G2 & Control & Active suspension & Whole gut & 21 & 2 & 2 \\
\hline 42 & C2.D21.G3 & Control & Active suspension & Whole gut & 21 & 2 & 3 \\
\hline 43 & C2.D21.G4 & Control & Active suspension & Whole gut & 21 & 2 & 4 \\
\hline 44 & C3.D21.G1 & Control & Active suspension & Whole gut & 21 & 3 & 1 \\
\hline 45 & C3.D21.G2 & Control & Active suspension & Whole gut & 21 & 3 & 2 \\
\hline 46 & C3.D21.G3 & Control & Active suspension & Whole gut & 21 & 3 & 3 \\
\hline 47 & C3.D21.G4 & Control & Active suspension & Whole gut & 21 & 3 & 4 \\
\hline 48 & C1.D28.G1 & Control & Active suspension & Whole gut & 28 & 1 & 1 \\
\hline 49 & C1.D28.G2 & Control & Active suspension & Whole gut & 28 & 1 & 2 \\
\hline 50 & C1.D28.G3 & Control & Active suspension & Whole gut & 28 & 1 & 3 \\
\hline 51 & C1.D28.G4 & Control & Active suspension & Whole gut & 28 & 1 & 4 \\
\hline 52 & C2.D28.G1 & Control & Active suspension & Whole gut & 28 & 2 & 1 \\
\hline 53 & C2.D28.G2 & Control & Active suspension & Whole gut & 28 & 2 & 2 \\
\hline 54 & C2.D28.G3 & Control & Active suspension & Whole gut & 28 & 2 & 3 \\
\hline 55 & C2.D28.G4 & Control & Active suspension & Whole gut & 28 & 2 & 4 \\
\hline 56 & C3.D28.G1 & Control & Active suspension & Whole gut & 28 & 3 & 1 \\
\hline 57 & C3.D28.G2 & Control & Active suspension & Whole gut & 28 & 3 & 2 \\
\hline 58 & C3.D28.G3 & Control & Active suspension & Whole gut & 28 & 3 & 3 \\
\hline 59 & C3.D28.G4 & Control & Active suspension & Whole gut & 28 & 3 & 4 \\
\hline 60 & P1.D14.G1 & Probiotic & Probiotic chamber & Whole gut & 14 & 1 & 1 \\
\hline 61 & P1.D14.G2 & Probiotic & Probiotic chamber & Whole gut & 14 & 1 & 2 \\
\hline 62 & P2.D14.G1 & Probiotic & Probiotic chamber & Whole gut & 14 & 2 & 3 \\
\hline 63 & P2.D14.G2 & Probiotic & Probiotic chamber & Whole gut & 14 & 2 & 4 \\
\hline 64 & P1.D21.G1 & Probiotic & Active suspension & Whole gut & 21 & 1 & 1 \\
\hline 65 & P1.D21.G2 & Probiotic & Active suspension & Whole gut & 21 & 1 & 2 \\
\hline 66 & P1.D21.G3 & Probiotic & Active suspension & Whole gut & 21 & 1 & 3 \\
\hline 67 & P1.D21.G4 & Probiotic & Active suspension & Whole gut & 21 & 1 & 4 \\
\hline 68 & P2.D21.G1 & Probiotic & Active suspension & Whole gut & 21 & 2 & 1 \\
\hline 69 & P2.D21.G2 & Probiotic & Active suspension & Whole gut & 21 & 2 & 2 \\
\hline 70 & P2.D21.G3 & Probiotic & Active suspension & Whole gut & 21 & 2 & 3 \\
\hline 71 & P2.D21.G4 & Probiotic & Active suspension & Whole gut & 21 & 2 & 4 \\
\hline 72 & P3.D21.G1 & Probiotic & Active suspension & Whole gut & 21 & 3 & 1 \\
\hline 73 & P3.D21.G2 & Probiotic & Active suspension & Whole gut & 21 & 3 & 2 \\
\hline 74 & P3.D21.G3 & Probiotic & Active suspension & Whole gut & 21 & 3 & 3 \\
\hline 75 & P3.D21.G4 & Probiotic & Active suspension & Whole gut & 21 & 3 & 4 \\
\hline 80 & P1.D28.G1 & Probiotic & Active suspension & Whole gut & 28 & 1 & 1 \\
\hline 81 & P1.D28.G2 & Probiotic & Active suspension & Whole gut & 28 & 1 & 2 \\
\hline 82 & P1.D28.G3 & Probiotic & Active suspension & Whole gut & 28 & 1 & 3 \\
\hline 83 & P1.D28.G4 & Probiotic & Active suspension & Whole gut & 28 & 1 & 4 \\
\hline 84 & P2.D28.G1 & Probiotic & Active suspension & Whole gut & 28 & 2 & 1 \\
\hline 85 & P2.D28.G2 & Probiotic & Active suspension & Whole gut & 28 & 2 & 2 \\
\hline 86 & P2.D28.G3 & Probiotic & Active suspension & Whole gut & 28 & 2 & 3 \\
\hline 87 & P2.D28.G4 & Probiotic & Active suspension & Whole gut & 28 & 2 & 4 \\
\hline 88 & P3.D28.G1 & Probiotic & Active suspension & Whole gut & 28 & 3 & 1 \\
\hline 89 & P3.D28.G2 & Probiotic & Active suspension & Whole gut & 28 & 3 & 2 \\
\hline 90 & P3.D28.G3 & Probiotic & Active suspension & Whole gut & 28 & 3 & 3 \\
\hline 91 & P3.D28.G4 & Probiotic & Active suspension & Whole gut & 28 & 3 & 4 \\
\hline
\end{tabular}


Table S5.3. Similarity percentages (SIMPER) analysis of gut microbiota on day 14. Table indicates the foremost three characteristic OTUs from each group contributing to the discrimination between the control and probiotic treatment. Contribution values indicate the importance of each OTU (percentage) in increasing Bray Curtis dissimilarity between the two treatments.

\begin{tabular}{|c|c|c|c|c|c|}
\hline \multicolumn{6}{|c|}{$\begin{array}{l}\text { Similarity Percentages - species contribu } \\
\text { Data type: Abundance } \\
\text { Resemblance: Bray-Curtis dissimilarity }\end{array}$} \\
\hline \multicolumn{6}{|c|}{ Groups Control \& Probiotic } \\
\hline \multicolumn{6}{|c|}{ Average dissimilarity $=\mathbf{9 6 . 8 2}$} \\
\hline \multirow{2}{*}{ Genus (OTUs) } & Group Control & Group Probiotic & & & \\
\hline & Av. Abundance & Av. Abundance & Av. Dissimilarity & Contrib\% & Cum.\% \\
\hline Bacillus (814) & 0 & 47.95 & 23.97 & 24.76 & 24.76 \\
\hline Nocardia (754) & 12.45 & 0.12 & 6.16 & 6.37 & 31.13 \\
\hline Mycobacterium (643) & 11.53 & 0.18 & 5.67 & 5.86 & 36.99 \\
\hline Rhodococcus (741) & 9.21 & 0 & 4.61 & 4.76 & 41.75 \\
\hline Rhodanobacter (340) & 0.34 & 8.2 & 3.94 & 4.07 & 45.81 \\
\hline Halomonas (496) & 0.25 & 3.6 & 1.69 & 1.74 & 47.55 \\
\hline
\end{tabular}

Table S5.4. Permutational MANOVA test and pairwise comparisons of gut microbiota for main factors effect and interaction terms. Analysis is based on Bray Curtis similarity of square root transformed relative abundance data. Permutation method was used on unrestricted permutations of raw data and 999 permutations. A pseudo- $F$ statistic was computed for each permutation and the $\mathrm{P}$ (perm) values give the proportion of permuted pseudo- $\mathrm{F}$ statistics that are equal to or greater than the original (un-permuted) pseudo- $F$ statistic. A multivariate analogue to the univariate t-statistic was used for the pairwise comparisons. The effect on gut microbiota was tested for factors "treatment" (control and probiotic) and "day" (day 21 and 28). df: Degrees of freedom, SS and MS: Sum and mean of the squares.

\begin{tabular}{|c|c|c|c|c|c|c|}
\hline \multicolumn{7}{|c|}{ PERMANOVA main test results } \\
\hline Source & df & SS & MS & Pseudo-F & $\mathbf{P}($ perm) & Unique perms \\
\hline Treatment & 1 & 7269.3 & 7269.3 & 8.8805 & 0.001 & 998 \\
\hline Day & 1 & 6784.7 & 6784.7 & 8.2885 & 0.001 & 999 \\
\hline Treatment x Day & 1 & 2036.1 & 2036.1 & 2.4874 & 0.012 & 999 \\
\hline Residual & 44 & 36017 & 818.57 & & & \\
\hline Total & 47 & 52107 & & & & \\
\hline \multicolumn{7}{|c|}{ PERMANOVA pairwise comparisons } \\
\hline \multicolumn{7}{|c|}{ Term 'Treatment x Day' for pairs of levels of factor 'Treatment' } \\
\hline \multicolumn{7}{|c|}{ Within level 'Day 21' of factor 'Day' } \\
\hline Groups & df & $\mathbf{t}$ & & rm) & Unique perr & \\
\hline Control vs. Probiotic & 22 & 2.1573 & & & 996 & \\
\hline \multicolumn{7}{|c|}{ Within level 'Day 28' of factor 'Day' } \\
\hline Control vs. Probiotic & 22 & 2.5883 & & & 999 & \\
\hline \multicolumn{7}{|c|}{ Term 'Treatment x Day' for pairs of levels of factor 'Day' } \\
\hline \multicolumn{7}{|c|}{ Within level 'Control' of factor 'Treatment' } \\
\hline Day 21 vs 28 & 22 & 1.9031 & & & 996 & \\
\hline \multicolumn{7}{|c|}{ Within level 'Probiotic' of factor 'Treatment' } \\
\hline Day 21 vs 28 & 22 & 2.6979 & & & 998 & \\
\hline
\end{tabular}


Table S5.5. Multivariate permutation dispersion (PermDisp) testing for heterogeneity of community structure within each group. Analysis is based on square root relative abundance OTU data. Analysis tested the null hypothesis of no difference between groups' dispersion by computing the dissimilarity of each sample from its group centroid and assessing by permutation analysis whether these dissimilarities differ between the two groups. Significant effects on group dispersion were tested for factor "Treatment" (control \& probiotic) on experimental days 21 and 28 .

\begin{tabular}{|c|c|c|c|c|}
\hline $\begin{array}{l}\text { Day } 21 \text { of factor } \\
\text { "Treatment" }\end{array}$ & F-statistic & Sample Size & \multicolumn{2}{|c|}{ Average Dispersion } \\
\hline Control & & 12 & \multicolumn{2}{|c|}{$28.25(0.73)$} \\
\hline Probiotic & & 12 & \multicolumn{2}{|c|}{$23.84(1.32)$} \\
\hline Deviation from centroid & & $\mathrm{t}$ & df & $\mathrm{P}$ (perm) \\
\hline Control vs. Probiotic & 8.4965 & 2.915 & 22 & 0.011 \\
\hline $\begin{array}{l}\text { Day } 28 \text { of factor } \\
\text { "Treatment" }\end{array}$ & F-statistic & Sample Size & \multicolumn{2}{|c|}{ Average Dispersion } \\
\hline Control & & 12 & \multicolumn{2}{|c|}{$27.22(0.81)$} \\
\hline Probiotic & & 12 & \multicolumn{2}{|c|}{$24.79(1.21)$} \\
\hline Deviation from centroid & & $\mathrm{t}$ & $\mathrm{df}$ & $\mathrm{P}$ (perm) \\
\hline Control vs. Probiotic & 2.7827 & 1.6681 & 22 & 0.121 \\
\hline
\end{tabular}

Table S5.6. Permutational MANOVA table testing for differences in water microbiota between the two treatments on each experimental day. Analysis was based on Bray Curtis similarity of square root transformed relative abundance data. Permutation method was used on unrestricted permutations of raw data. A pseudo- $F$ statistic was computed for each permutation and the $P$ (perm) values give the proportion of permuted pseudo- $F$ statistics that are equal to or greater than the original (un-permuted) pseudo-F statistic. SS and MS: Sum and mean of the squares, df: degrees of freedom, P(MC): P-values calculated based on 9999 Monte Carlo permutations drawn from the theoretical asymptotic permutation distribution.

\begin{tabular}{lccccccc}
\hline \multicolumn{7}{c}{ PERMANOVA table for factor “Treatment” (Probiotic vs Control) } \\
\hline $\begin{array}{l}\text { Water Day 14 } \\
\text { Source }\end{array}$ & df & SS & MS & Pseudo-F & P(perm) & Unique Perms & P(MC) \\
Treatment & 1 & 1440.9 & 1440.9 & 1.3695 & $\mathbf{0 . 1 9}$ & 10 & $\mathbf{0 . 2 9}$ \\
Residual & 4 & 4208.3 & 1052.1 & & & & \\
Total & 5 & 5649.2 & & & & & \\
Water Day 21 & & & & & & & \\
Source & df & SS & MS & Pseudo-F & P(perm) & Unique Perms & P(MC) \\
Treatment & 1 & 1197 & 1197 & 1.2964 & $\mathbf{0 . 3 1 3}$ & 10 & $\mathbf{0 . 3 1 1}$ \\
Residual & 4 & 3693.3 & 923.33 & & & & \\
Total & 5 & 4890.4 & & & & & \\
Water Day 28 & & & & & & & \\
Source & df & SS & MS & Pseudo-F & P(perm) & Unique Perms & P(MC) \\
Treatment & 1 & 1440.9 & 1440.9 & 1.3695 & $\mathbf{0 . 1 9}$ & 10 & $\mathbf{0 . 2 9}$ \\
Residual & 4 & 4208.3 & 1052.1 & & & & \\
Total & 5 & 5649.2 & & & & & \\
\hline
\end{tabular}



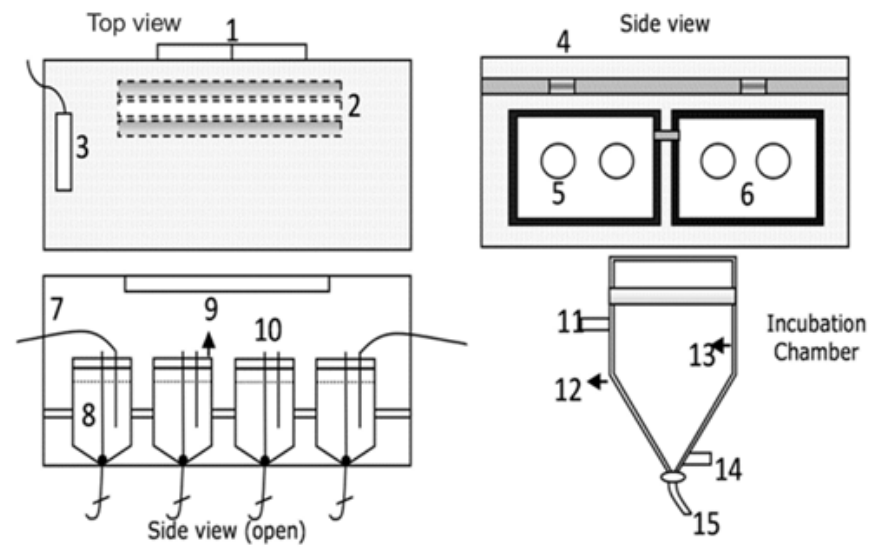

Figure S5.1. Customized cabinet used in probiotic stage. Dimensions LxWxH: 1500x65x70 (mm). (1) HEPA filter $(0.3 \mu \mathrm{m}),(2)$ UV ( 1 x 30W Ultraviolet $253.7 \mathrm{~nm}$ ) and daylight (TLD-30W) lamps; (3) Built-in electric sockets, (4) Airtight locks, (5) Gloves, (6) Plexiglas doors (UV-protection), (7) air flow inlet (0.25 $\mu \mathrm{m}$ filter), (8) Custom-built glass chamber (incubation), $\varnothing 12 \mathrm{~cm}, \mathrm{~V}: 2 \mathrm{~L}$, (9) PVC chamber lid $\varnothing 16 \mathrm{~cm},(10)$ Bottom sieve remover, (11) Heated water inlet, (12 \& 13) Outer/inner double wall glass, (14) Heated water outlet and (15) Discharge valve. 


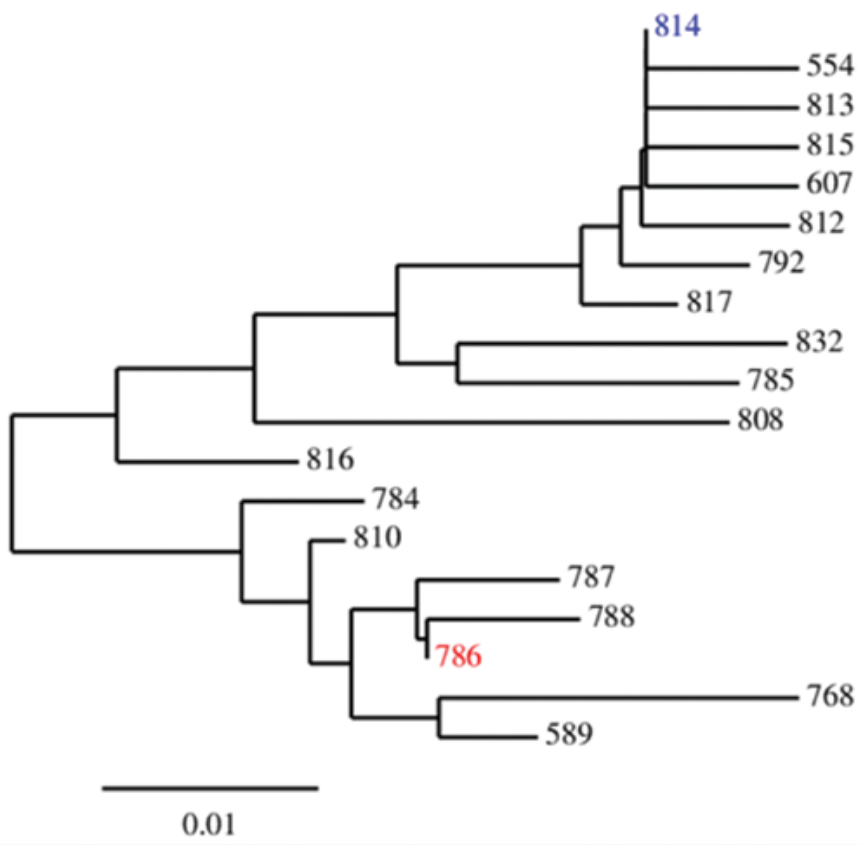

BLASTN 2.2.31+

RID: NOXKAKB9014

Database: Nucleotide collection ( $\mathrm{nt}$ )

$29,980,884$ sequences; $93,445,391,531$ total letters

\begin{tabular}{|c|c|c|}
\hline \multicolumn{3}{|l|}{ OTU $=814$} \\
\hline \multicolumn{3}{|l|}{ Length $=141$} \\
\hline Sequences producing significant alignments: & Score E & (Bits) Value \\
\hline gb|KF318848.1| Bacillus subtilis subsp. subtilis strain I12 1... & 134 & $3.00 E-28$ \\
\hline gb|KF318844.1| Bacillus subtilis subsp. subtilis strain I8 16... & 134 & $3.00 E-28$ \\
\hline gb|KF318817.1| Bacillus subtilis subsp. subtilis strain I51 1... & 134 & 3.00E-28 \\
\hline gb|KF318806.1| Bacillus subtilis strain I45 16S ribosomal RNA... & 131 & $3.00 E-27$ \\
\hline \multicolumn{3}{|l|}{ OTU= 786} \\
\hline \multicolumn{3}{|l|}{ Length=141 } \\
\hline Sequences producing significant alignments: & Score E & (Bits) Value \\
\hline gb|KM205823.1| Bacillus sp. RB05 16S ribosomal RNA gene, part... & 136 & $8.00 E-29$ \\
\hline gb|KJ606963.1| Uncultured bacterium clone Aer5_12C_T307 16S r... & 131 & $3.00 E-27$ \\
\hline gb|CP009628.1| Bacillus cereus ATCC 4342, complete genome & 131 & $3.00 E-27$ \\
\hline gb|KP142408.1| Uncultured bacterium clone PT_1 16S ribosomal ... & 131 & $3.00 E-27$ \\
\hline gb|JX901106.1| Bacillus cereus strain 8B 16S ribosomal RNA ge... & 131 & $3.00 E-27$ \\
\hline
\end{tabular}

Figure S5.2. Phylogenetic tree phylogram of Bacillus related OTUs and BLAST analysis results for the two most abundant OTUs. Numbers at the branch's end indicate the OTU ID. OTU 814 (blue) is the added B. subtilis and 786 (red) is the second most abundant OTU member of the genus Bacillus belonging to another species, as indicated in the table. Tree branches give an estimate of a phylogeny where branch lengths are proportional to the amount of inferred evolutionary change. 


\title{
Chapter 6
}

\author{
General Discussion
}

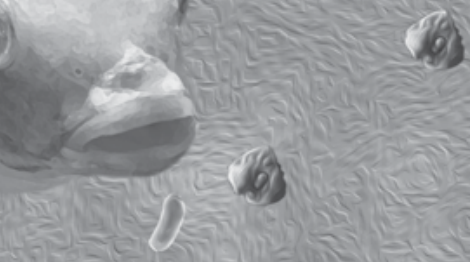

(6)

48

(2)
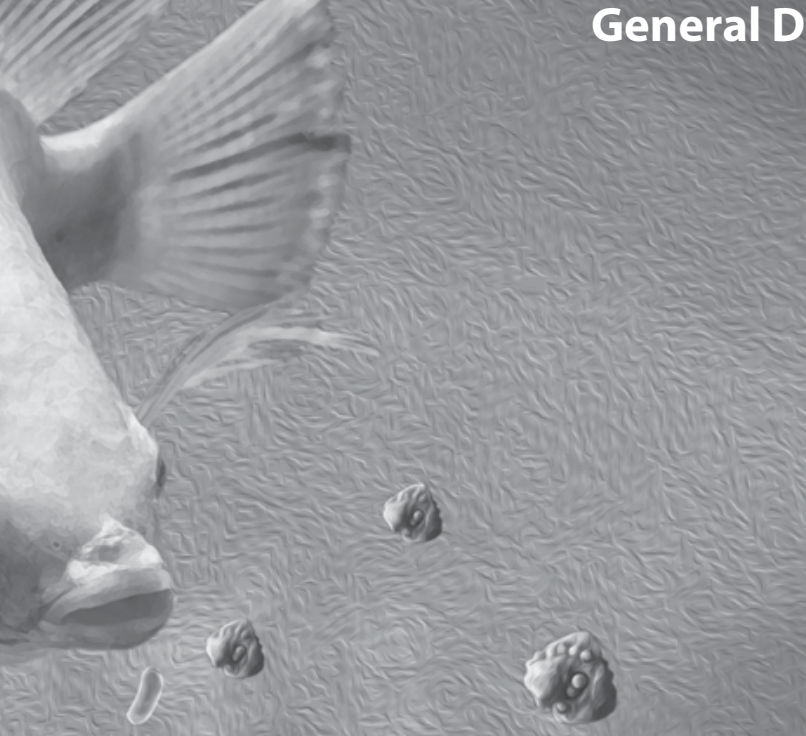


\subsection{Introduction}

Aquaculture has been has evolved as the fastest growing food-producing sector over the last 20 years (FAO, 2014). Nevertheless, the aquaculture production sector is still facing major bottlenecks such as suboptimal growth and high mortality (Attramadal et al., 2012). Fish-microbe interaction has been suggested to be a key factor related to the aforementioned limitations (Vadstein et al., 2013). Exploration of the possible beneficial roles of intestinal microbiota in fish started very recently looking into various aspects, such as nutrient metabolism, innate immune response and epithelial proliferation (Roeselers et al., 2011).

The underlying mechanisms that drive spatio-temporal dynamics of abundance and diversity of intestinal microbiota have not received sufficient attention. Traditional microbial management techniques (e.g. antibiotics, hygiene protocols), and more recently probiotic administration, have been applied empirically (Martínez Cruz et al., 2012) and still lack adequate and consistent scientific evidence to validate the proposed health benefits of microbial management. In order to design innovative and effective microbial management strategies, we need to elucidate the metabolic potential of microbial diversity, disentangle the host-microbe interactions and clarify the environmental impact on gut microbial assembly.

Intestinal colonization is largely a stochastic process, with successful bacteria being those that happened to be present at the right moment and place (Fjellheim et al., 2007). However, environmental, nutritional and physiological influences can lead to the disturbance of diversity, abundance and/or functionality of the gut microbiota (Verschuere et al., 1997; De Schryver and Vadstein, 2014). The microbial composition in the gut is also influenced by microbiota in the environment and by the host itself (Rawls et al., 2006; Navarrete et al., 2012; Verschuere et al., 2000).

The main objective of this thesis was to deepen our understanding on the principal factors driving gut microbiota development. In particular, we determined the impact of rearing environment (e.g. free-living bacteria), early life microbial exposure and probiotic use on the microbial composition and abundance inside the gut of developing tilapia larvae. The sequencing libraries presented in this thesis represent the most comprehensive collection of $16 \mathrm{~S}$ rRNA gene sequences from gut microbiota of a commercial fish species and its environment. This chapter discusses the research needed to implement more sustainable and applicable microbial management strategies for aquaculture. In addition, a metaanalysis is presented in which our main findings are compared to publically available gut microbiome datasets of different species. In this way, we aimed to provide a broader perspective on the forces that structure gut microbiota, including free-living communities from different environments. 


\subsection{Temporal variation of gut microbiota}

The gut microbiota of fish larvae evolves fast into a complex and dynamic community, which differs by location in the gastro-intestinal tract. Differences in the microbial communities over time are defined as temporal variation. In chapter $\mathbf{2}$ we showed that time was the most significant factor related to microbial shifts in the fish gut. Temporal variation of fish gut microbiota is more pronounced during early development when the fish gut is not yet fully developed and the immune system is immature (Gatesoupe, 1999). Thus, early microbial fluctuations in the fish gut are primarily driven by ontogenetic changes (i.e. genetic, physiological), whereas adaptation to nutritional and environmental situations should be expected at later developmental stages (Nayak, 2010b; Romero and Navarrete, 2006; Navarrete et al., 2012; Li et al., 2012b).

The observed rapid and stochastic changes of microbiota in the gut over time suggest that results should be interpreted carefully since observations of start and endpoint do not provide information about the temporal variation in between. Additionally, ontogeny associated to the alimentary canal (morphology and function), immune system, feeding habits (behavior and type) and habitat preference (fresh or saltwater, pelagic or demersal) can be very different between fish species (Hansen and Olafsen, 1999; Bowden et al., 2005; Zapata et al., 2006; Tytler et al., 1990; Noakes and Barlow, 1976; German et al.). Thus, ontogenetic traits should be considered carefully when gut microbial shifts are studied over different developmental stages or between different fish species.

\subsection{The impact of feed and water microbiota on gut microbial composition}

Regarding within rearing system tank-to-tank variation, our results suggest that variation of gut microbiota between individuals is low as far as fish receive the same diet and share the same water (Chapter 2). On the contrary, studies with marine species show that variation between identically reared individuals could be higher than the one observed here (Bakke et al., 2013; Fjellheim et al., 2011; Gatesoupe and Covès, 2012). This can possibly be attributed to the fact that in the studies with marine species larvae are fed live feed, which was not the case in our studies. Since feed associated bacteria affect gut microbiota in fish larvae (Mclntosh et al., 2008; Gatesoupe and Covès, 2012; Brunvold et al., 2007), higher batch-to-batch variation should be expected in larvae fed live feed, compared to larvae receiving formulated feed.

Water and feed microbiota are the only two microbial sources for fish. We found that gut microbiota from individuals reared in replicate production systems differed significantly even though systems were handled identically and larvae were fed the same feed. Observed differences of water microbiota between replicate systems is the only plausible 
explanation for the observed differences in the fish gut communities. Therefore, we recommend to investigate treatment effects (e.g. different diets) on gut microbiota within the same system (fish share the same water source), rather than between replicate systems. In fish production facilities, this would mean that producers should not always expect similar microbial composition neither in the gut of fish nor in the water of systems managed similarly.

The effect of different rearing techniques i.e. recirculating (RAS) vs active suspension (AS) systems on the bacterial community composition of the larvae was evaluated in chapters $\mathbf{2}$ and $\mathbf{3}$. We found that the choice of culture system strongly determined the composition and development of bacterial communities in the water, which in turn, triggered changes in the fish gut communities. Our data showed that the water microbiota had a greater impact on the composition of gut communities than feed microbiota, which concurs with the findings of Bakke et al. (2013). These results can be of great significance for aquaculture production as the choice of production system could, to a certain degree, determine the composition of the microbiota in the gut. Thus, one could explore the possibility of water microbial management customized for each production system (e.g. use of probiotics) to realize health benefits for the host and/or environmental benefits by improving water quality.

Stochastic factors also influenced early colonization in the gut. Stochastic phenomena imply that the composition of the microbiota is determined by probability. Because of this stochasticity, sampling power and pooling of samples should be taken into consideration. To obtain a reliable representation of the gut microbial composition, sufficient numbers of individuals need to be sampled. In many studies, individual gut or larvae samples are pooled, running the risk that inter-individual variation could be completely masked. Analyzing individuals is recommended as long as the causes for the inter-individual variability are insufficiently understood. The minimum sample size for hypothesis testing should be estimated from beforehand and the method used for such power calculations should be explained carefully in scientific papers and reports.

The small impact of feed microbiota compared to the impact of water microbiota on gut microbiota found in chapters $\mathbf{2}$ and $\mathbf{3}$ could be a consequence of the fact that tilapia larvae were fed artificial diets that all passed through the same processing steps and which were partially sterilized during processing (e.g. heating, extrusion). However, when deliberately making large contrasts in feed microbiota by mixing commercial feed with different types of sludge as described in chapter 4, feed-associated bacteria did affect the gut microbiota. Contrarily to the previous chapters, gut microbiota shared a much higher number of OTUs (operational taxonomic units) with feed than with water microbiota. There are two elements which might explain this outcome. First, the microbial load of the sludge-based diets used in the chapter 4 was higher and more diverse compared to 
the commercial diet. This could explain why gut microbiota of larvae fed the control diet were mainly influenced by the water rather than by the feed microbiota. Second, to make the sludge-based diets, sludge produced under aerobic, methanogenic and denitrifying conditions was mixed with the commercial diet, which resulted in a higher relative species abundance and diversity in feed microbiota than for the water microbiota. Therefore, we believe that the level of (environmental) influence on the gut microbiota highly depends on the degree of diversity and abundance of the microbiota in the environment as well as the metabolic and ecological compatibility of the latter inside the fish gut.

In a study with chicken, Yin et al., (2010) suggested that, at a very early stage of life, bacterial succession in the gut can be influenced by a combination of exposure to proper bacterial inocula and host selective pressures. Depending on the production system, fish can have access to taxonomically and metabolically diverse microbial communities which in turn have a higher or lower chance in adapting to the gut habitat. Nile tilapia has an omnivorous feeding strategy including detritivory (El-Sayed, 2006). The close association with sediments during feeding may suggest that sediment associated microbes are taken up by Nile tilapia. The fact that anaerobic (obligate and facultative) bacteria are among the principal colonizers of the fish gut indicates the preference of these bacterial groups for anaerobic habitats. To which extent species in sediment microbiota could successively establish themselves in the fish gut, depends on their adaptability within the gut environment and the host's preferences. Our experiment with sludge-based diets suggests to further exploring this route.

Summarizing, this shows that the microbial assembly in the fish gut is a skewed selection of feed and water bacteria, as a result of a random sampling from the available environmental bacterial pool, determined by the historical contingency (timing and order of species inflow), species interactions within the gut and host preferences. Understanding how microbiota in water, feed and gut interact still needs further research. Insights are still too limited to be applied in improving the design, microbial management and nutrient cycling of fish rearing systems. Future research should first focus on disentangling the key processes governing selection and propagation of water and feed microbiota, which ultimately will become important species of the microbial community in the adult fish gut. 


\subsection{The impact of probiotic use on gut composition during early development}

Probiotics have been shown to reduce the risk and severity of infections caused by bacterial pathogens (Gatesoupe, 1999; Martínez Cruz et al., 2012) and have been successfully used as immunostimulants (Cain and Swan, 2010; Lepage et al., 2012; Song et al., 2014) and as growth promoters in fish and shrimp (Pérez-Sánchez et al., 2014; Merrifield et al., 2010). Currently, delivery of probiotics through feed in larval stages is the most common strategy for management of larval gut microbiota in aquaculture (Nayak, 2010a). Here, the successful transfer of a probiotic strain via the water into the gut indicates that inoculation of the gut community with bacteria during early gut development is conceivable. To enhance the colonization success of the probiotic inside a "virgin" gut ecosystem, we maintained the larvae under axenic conditions before exposure to a probiotic strain. Although for research purposes the use of gnotobiotic or axenic systems is an excellent tool to extend our understanding on host-microbe interactions and microbial community assembly, in commercial production of fish larvae maintaining axenic conditions is practically impossible. Nevertheless, an early application of probiotics during the yolk sac stage could increase the chances for a successful colonization of the larval gut.

Further, in chapter $\mathbf{5}$ we observed two interesting patterns. First, the final concentration of probiotic cells in the water was different between replicate tanks, although the same number of probiotic cells was added daily. Second, the gut microbiota of the larvae was always dominated by $B$. subtilis, the probiotic administrated in our study. These findings demonstrate that application of probiotics through water led to successful transfer of the strain into the gut. Nevertheless, the abundance in the water was not a good predictor of the abundance in the fish gut.

After discontinuing the administration of the probiotic strain, its abundance declined. This could indicate a lack of adaptability of the probiotic strain inside the gut and/or strong host selectivity against the probiotic strain. It means that successful colonization of a probiotic strain in the fish gut remains very challenging. The presence of the strain in the gut can only be expected during the period of supplementation or shortly after discontinuation (Kim and Austin, 2006; Vaseeharan and Ramasamy, 2003; Sharifuzzaman et al., 2014; Zhang et al., 2009; Robertson et al., 2000). Dosage and duration of probiotic supplementation may influence colonization success, but the persistence of the probiotic after entering the gut remains largely a black box. Possible factors influencing persistence include the probiotic species, the host species, environmental factors and the developmental stage of the fish (Pérez et al., 2010; Ramos et al., 2013; Standen et al., 2015). Following a similar approach as in fecal microbiota transplantation (FMT), (Khoruts and Sadowsky, 2011), transmitting donor phenotypes of interest could help us understand the mechanisms of colonization and persistence of microbial transplants in the recipient gut. 
Regardless of the low survival of the probiotic strain, gut microbiota remained different between treatments even after the discontinuation of probiotic administration and even though the fish were receiving the same diet and swimming in water with a similar microbial profile. The initial presence of $B$. subtilis may have led to a different sequence of events of bacterial colonization due to synergistic or antagonistic interactions the probiotic strain and/or other bacteria entering the gut. We suggest that future studies should focus on the long term effects of probiotic exposure during early developmental stages of fish. It has been suggested that in humans, legacy effects can play a role in defining microbial structure, but that these effects can be quickly minimized by the diet of the host (Turnbaugh et al., 2009b). If this applies also to fish, we suggest that early administration of a probiotic strain/s during the larval stage, accompanied by a continuous prebiotic administration (synbiotics) or dietary management could further extend the probiotic residence in the gut even after its discontinuation.

\subsection{Meta-analyses of the fish gut microbiota}

Several publications have focused on the impact of nutritional and environmental changes on fish gut microbial communities. In this context, the meta-analysis study of Sullam et al. (2012) has been a valuable contribution towards identifying factors determining microbial assembly inside the fish gut. Authors suggested that gut microbial composition is strongly correlated with species trophic level and habitat salinity. Comparison of gut microbiota with other habitats revealed that fish gut communities are often similar to those of other animals and rarely reflect the communities of their surrounding habitats. However, in that meta-analysis data from both culture-dependent and independent methods were used which could introduce apparent variation between samples and/or different studies.

To determine the relative importance of factors on shaping the microbial communities in the fish gut, we performed a meta-analysis of $16 \mathrm{~S}$ rRNA gene sequence data from 12 different studies on gut microbiota of different fish species. The meta-analysis incorporated datasets from the three studies included in this thesis, unpublished datasets from two of our previous studies on tilapia larvae and seven publically available gut microbiome datasets from studies performed in trout, carp, zebrafish and tilapia. Data in these studies were generated by using different sequencing platforms (454 Titanium, Illumina HiSeq 2000 and lon-torrent) and primers targeting different regions of the 16S rRNA gene. 


\subsubsection{Methodology}

Datasets were selected through a review of published studies on fish gut microbiota and their environments. Only data from freshwater species were used due to the lack of publically available data on gut microbiome of saltwater fish species generated by sequencing platforms other than Sanger sequencing. We initially analyzed datasets generated only by the Illumina sequencing platform i.e. from the two studies included in this thesis (chapter 3 and $\mathbf{4}$ ) and from another two of our unpublished studies on tilapia larvae. From that dataset we sought to determine the relationship between gut microbial communities and those present in their feed and water. The next step was to analyze all available datasets with sequences originating from the gut without including data from non-gut samples. We examined the phylogenetic similarity among gut bacteria of the same and different fish species from different habitats, diets, facilities and developmental stage. Sample metadata and study supplementary information are shown in Table S6.1, 6.2 and 6.3.

Sequencing data were analyzed using NG-Tax, an in-house pipeline for 16S rRNA amplicon analysis, including the prediction of sequence regions not covered by the original sequence data to improve comparability across different platforms (Ramiro Garcia et al., 2016). Statistical analyses were performed using Primer 6 (version 6.1.11) (Primer-E Ltd., Plymouth, United Kingdom) and its PERMANOVA add-on package (Gorley and Clarke, 2006).

\subsubsection{Relationships between gut, water and feed microbiota: Illumina dataset of this thesis}

We sought to determine the relationship between gut microbial communities of tilapia larvae with those present in the water and feed. Unweighted Unifrac was used to examine phylogenetic distance between fish gut and environmental microbial communities. Principal coordinates analysis ( $P C O A$ ) showed that communities from different environments i.e. gut, water, and feed were significantly different (PERMANOVA test: $P_{\text {perm }}=0.001$ ), (Fig. 6.1a). We also found that gut microbial communities were less dispersed than the corresponding water or feed communities between experiments $\left(P_{\text {perm }}: 0.001\right)$. Within the illumina datasets, the lowest variation of gut communities was observed between samples from the probiotic study and the highest in the study where the sludge-based diets were used (Fig. 6.1b), $\left(\mathrm{P}_{\text {perm }}: 0.001\right)$. 

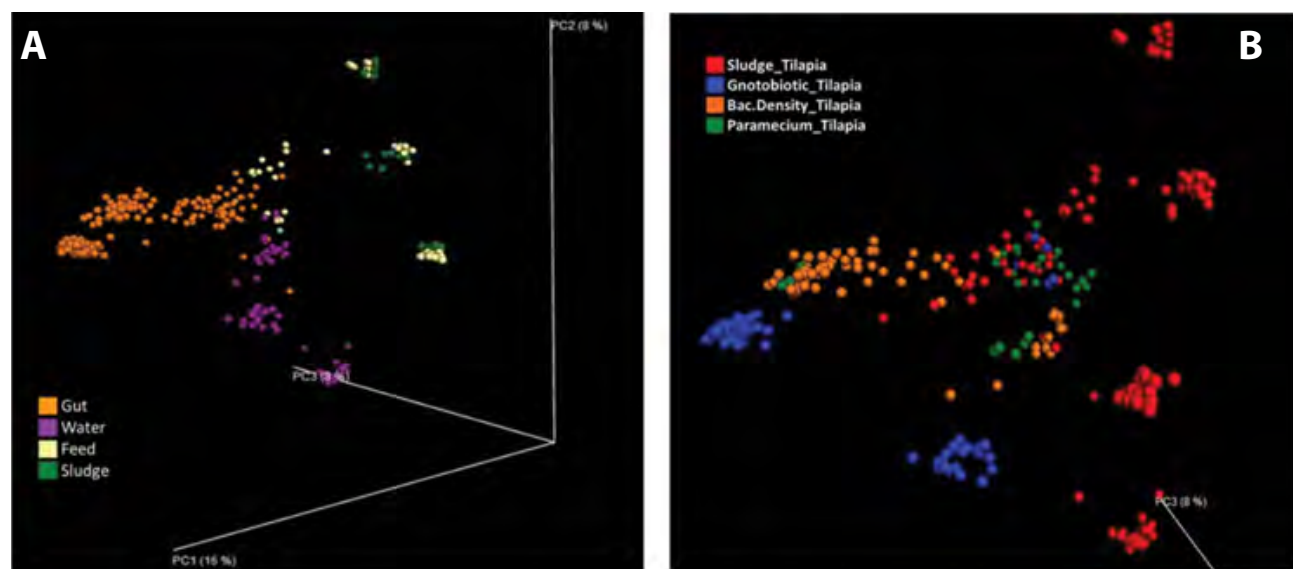

Figure 6.1 Principal coordinates analysis of microbiota from different sample types and studies. Relative distances of all points represent the relative dissimilarities of microbiota between samples as measured by Unweighted Unifrac. Spheres are color coded by: (A) Sample type; gut (orange), water (purple), feed (yellow) or sludge (green), and (B) Study; Sludge (red), Gnotobiotic (blue), Bac.Density (orange) or Paramecium (green). The percentage of total variation that is explained by each PCo axis is given in parentheses.

\subsubsection{Relationship of fish gut microbiota between different fish species}

A total of about $16.9 \mathrm{M}$ reads were selected from twelve next generation sequencing libraries on fish gut microbiota resulting in 2417 unique sequences. Among these sequences the dominant phylum (average abundance from all studies) was generally Proteobacteria (Fig. 6.2). In contrast, Fusobacteria was the most abundant phylum in the study of different carps raised in ponds (Li et al., 2014a), Firmicutes in the gut of tilapia fed with probiotics (Standen et al., 2015), and Actinobacteria, Planctomycetes and Firmicutes were the most dominant bacterial phyla in the gut of tilapia larvae in 3/5 of our studies i.e. the "Gnotobiotic", "Bac.Density" and "RAS-AS" studies (Fig. 6.2).

Based on unweighted Unifrac, a PCoA was performed and the 10 most discriminant genera were plotted in a biplot (Fig. 6.3). Gut samples from the probiotic study (Probiotics_Tilapia in Fig. 6.3) consisted mainly of species belonging to the genera Nocardia, Bacillus and Rhodococcus. In our unpublished study in tilapia larvae ("Bac.Density_Tilapia"), the larval gut was enriched with species from the genus Paenibacillus, whereas larvae fed with sludge-based diets ("Sludge_Tilapia") tended to have more species from the genus Ralstonia. From the studies which were principally discriminated along the PCoA1, species from the genera Bradyrhizobium and Sphingomonas (41 to 93\% of the total abundance) outnumbered all other species in the gut of rainbow trout, the only carnivore fish species of this dataset. 


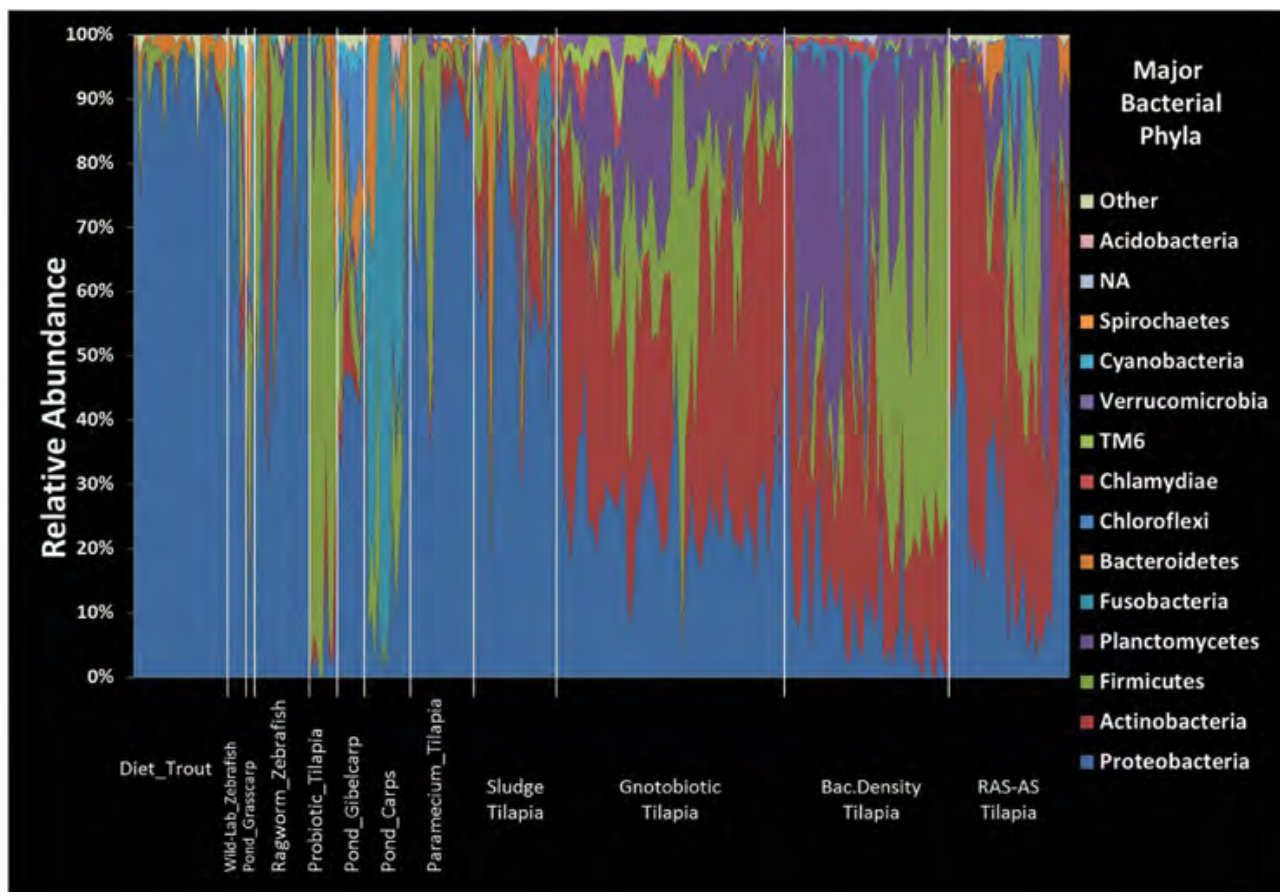

Figure 6.2. Area plot with percentage relative abundance of main bacterial phyla (y-axis, right) found in the gut samples of individuals from the different studies ( $\mathrm{x}$-axis)

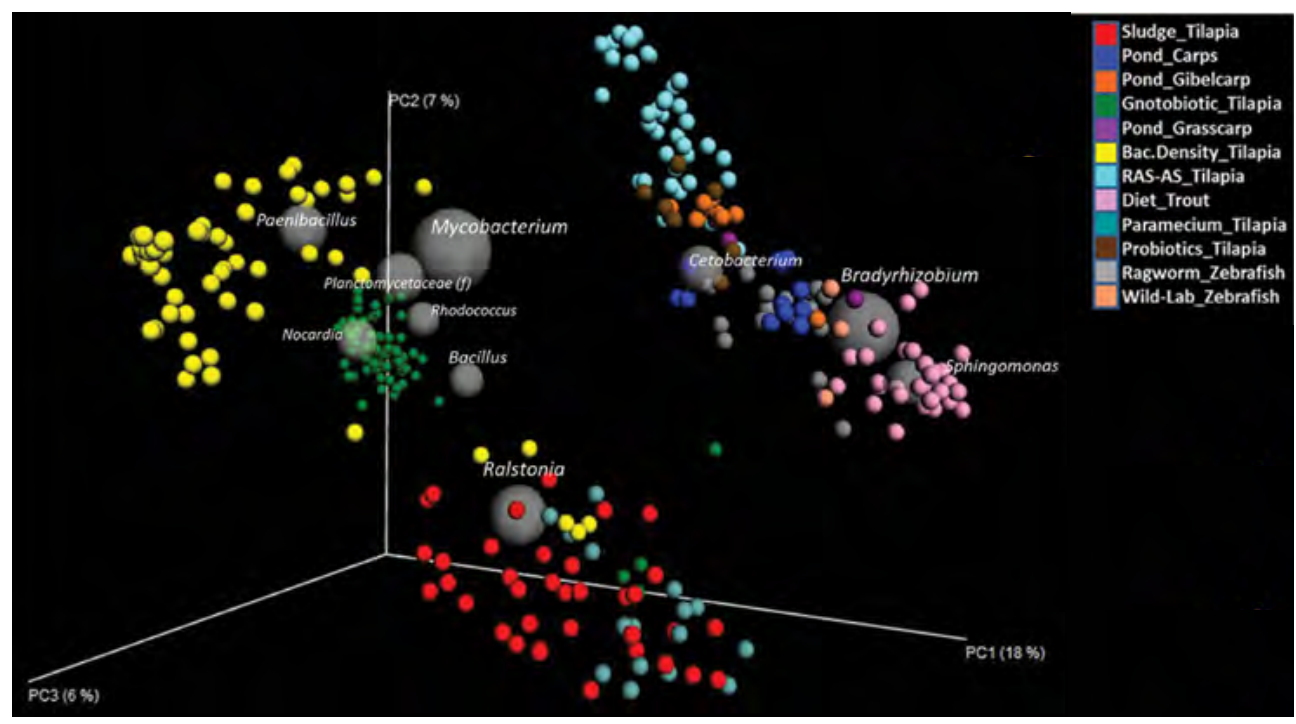

Figure 6.3. Principal coordinates biplot of gut microbiota of individuals from the different studies. Relative distances of all points represent the relative dissimilarities of gut microbiota between samples as measured by Unweighted Unifrac. Grey spheres represent the 10 most discriminant genera in explaining the observed variation between samples (colored spheres) and their size is proportional to the relative contribution of the genus in discriminating its space associated samples. The percentage of total variation that is explained by each PCo axis is given in parentheses. 


\subsubsection{Final Reflection}

The meta-analysis showed that the gut microbiota has only a limited overlap within those found in their surrounding environment. This is in agreement with Wong and Rawls, (2012) who stated that communities inside the gut do not directly reflect the ones present in their habitat. Obviously, the pressure responsible for the selection of microbiota in the gut highly depends on the host (Li et al., 2012b). Further, regardless of the different treatments applied across our studies, gut communities clustered closely together, compared to microbiota in water, feed and sludge. This indicates that gut is a habitat which is less amenable to manipulation than water or feed. The host contributes to a fairly stable environment in the gut, leading to a homeostatic interaction between host and gut microbiota. On the other hand, it also shows that strict selection criteria operate inside the gut regarding which bacteria flourish and which are extirpated. Habitat related selective pressures are attributed to $\mathrm{pH}$, gastric and bile secretions, nutrients availability and digestive enzymes activity, as well as host immune responses (Hansen and Olafsen, 1999; Verschuere et al., 1997; Sullam et al., 2012).

The lower variation of gut microbiota observed between samples from the probiotic study could be associated with the plasticity of gut microbiota in returning to a stable state after termination of a perturbation. Compared to the rest of our studies, the antibiotic and later probiotic treatments were terminated after one week, and from that point onwards, fish were receiving the same diet and swimming in water with a similar microbial profile. Thus, the time window of our observations could have coincided with the post-perturbation period of microbial community recovery. In that study, phylogenetically related genera tend to be abundant across most of the gut samples after discontinuation of the treatments. This resulted in a lower dispersion of gut samples when Unifrac distance was used (meta-analysis). Without considering the phylogenetic similarity of the taxa present in the gut, at a fine taxonomic resolution, gut microbiota was more clearly clustered between treatments as shown in chapter 5 . These results have both methodological and biological inference. Measures of $\beta$-diversity should be chosen carefully depending on the research question. Additionally, phylogenetically different bacteria can develop in the gut; yet, the functionality of these different communities might be highly similar, or vice versa. Literature regarding relationship between genetic and functional diversity of bacteria are controversial (Mouchet et al., 2012 and literature cited therein), indicating that future research should focus on unraveling this relationship in more detail.

The most abundant phylum in all analyzed studies was that of Proteobacteria, indicating that the majority of fish gut bacteria are members of this taxon. This corroborates the results of (Rawls et al., 2006; Nayak, 2010b). Apparently, Proteobacteria are well adapted to the fish gut habitat, despite phylogenetic differences between their hosts, habitat heterogeneity and differences in hypotheses tested, resulting in highly different treatments. Differences between the prevalent phyla were mostly due to the dominance of the phyla Actinobacteria, Planctomycetes and Firmicutes. These results disclose a separation between our own 
datasets and those acquired from other studies. Interlocation variation (variation between labs or facilities) of gut microbiota has been previously reported in zebrafish (Roeselers et al., 2011) but also in lab-reared mice (Friswell et al., 2010). The causes of the observed patterns could include differences in the choice of rearing system, water properties, handling and diets used.

It should be noted that almost half of the datasets incorporated in the meta-analysis were originated from our studies on tilapia larvae where the same methodology was used. This suggests that extrapolation of results from the meta-analysis presented here should be interpreted with caution due to the higher relative weight of the own work to the complete dataset. Nevertheless, within our own datasets spatial variation was observed between the "AS-RAS"-"Gnotobiotic"-“Bac.Density" and the "Paramecium"-"Sludge" studies. In particular, we found that Mycobacterium was the most discriminant genus in the larval gut of the first three studies. This difference could be attributed to the use of flow-through systems in the latter studies where the microbial load of the water was significantly reduced (lower carrying capacity due to water exchange and reduction of water microbial load due to UV sterilization).

Our phylogenetic analysis of gut communities revealed, in most cases, a clear clustering by study. To this end, differences in microbiota composition between experiments could be related to the experimental treatments and their effects on gut communities. However, also differences in methodological approaches could explain the differences found in microbiota composition. Choice of PCR primers, 16S rRNA region targeted, DNA extraction protocol, and sequencing platforms have been previously associated with significant differences between studies in human gut microbiome (Lozupone et al., 2013). It will therefore be important that future studies comparing gut microbiota of different fish species should be consistent with respect to the methods used for sample collection and analysis (Wong and Rawls, 2012).

Our results revealed a significant effect of rearing environment on gut microbiota. This observation was only valid within our facilities but not throughout the studies included in the meta-analysis. No significant effect of different rearing environments was observed in gut communities between animals raised in artificial or natural habitats (Wong and Rawls, 2012; Roeselers et al., 2011). In these studies, authors revealed that gut bacterial community membership in fish is mostly associated with their trophic level and matches their respective phylogenetic relationships, as has been also proposed for mammals (Ley et al., 2008). Here, we observed no shared constituents of the gut microbiota between the same fish species of the different studies. The clear dominance of the two genera (Bradyrhizobium and Sphingomonas) unique for the only carnivore fish species of this dataset (rainbow trout) could indicate host-microbe relationships attributed to the host's trophic level. Nonetheless, to draw clear conclusions it is important in the future to include additional fish from taxa, trophic levels and water salinities not included in this study. 


\subsection{Concluding remarks}

The aim of microbial management is to steer gut microbiota towards a desirable state. To do so, we need to understand the ecological mechanisms and forces in the larval gut that regulate community composition. The results presented in this thesis have contributed to this knowledge and facilitate the development of safe and effective methods for manipulating gut microbial composition to promote the health of fish. Guidelines for optimization of experimental design and methodological conformity are given together with suggestions and ideas for future research themes.

A major outcome of our work is that the initial microbial contact is leading in determining gut microbial assembly. This encourages the development of strategies to steer initial colonization through the microbial management of water and feed. Gut microbiota of larvae can be manipulated towards improved intestinal function and immune development through the application of probiotics at the critical early developmental stage. Live prey can be an effective way of delivering probiotics but also prebiotics to the larvae through bio-encapsulation and/or bio-enrichment. The use of a selected mixture of beneficial strains combined with dietary ingredients (synbiotics) may prove more effective in different situations and more stable over time compared to a single strain probiotic.

The reduction of wild fish stocks, the high demand for fish protein and the need to develop sustainable aquaculture production methods are challenges which offer also opportunities. The areas of research interest have been defined and the technology is slowly shifting towards the era that next generation sequencing will become routine. The coming years will be an exciting time for fish microbiome research. 


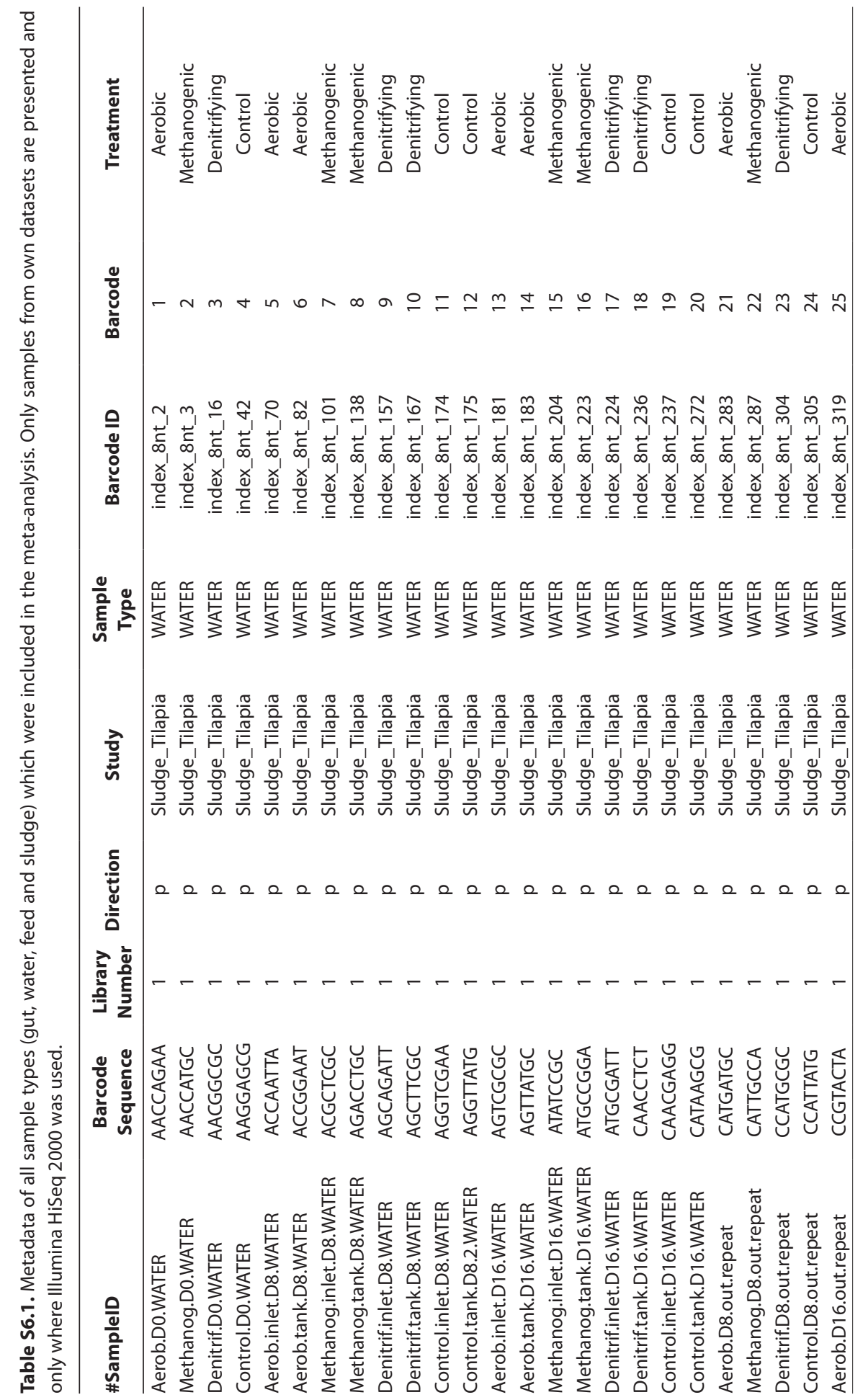




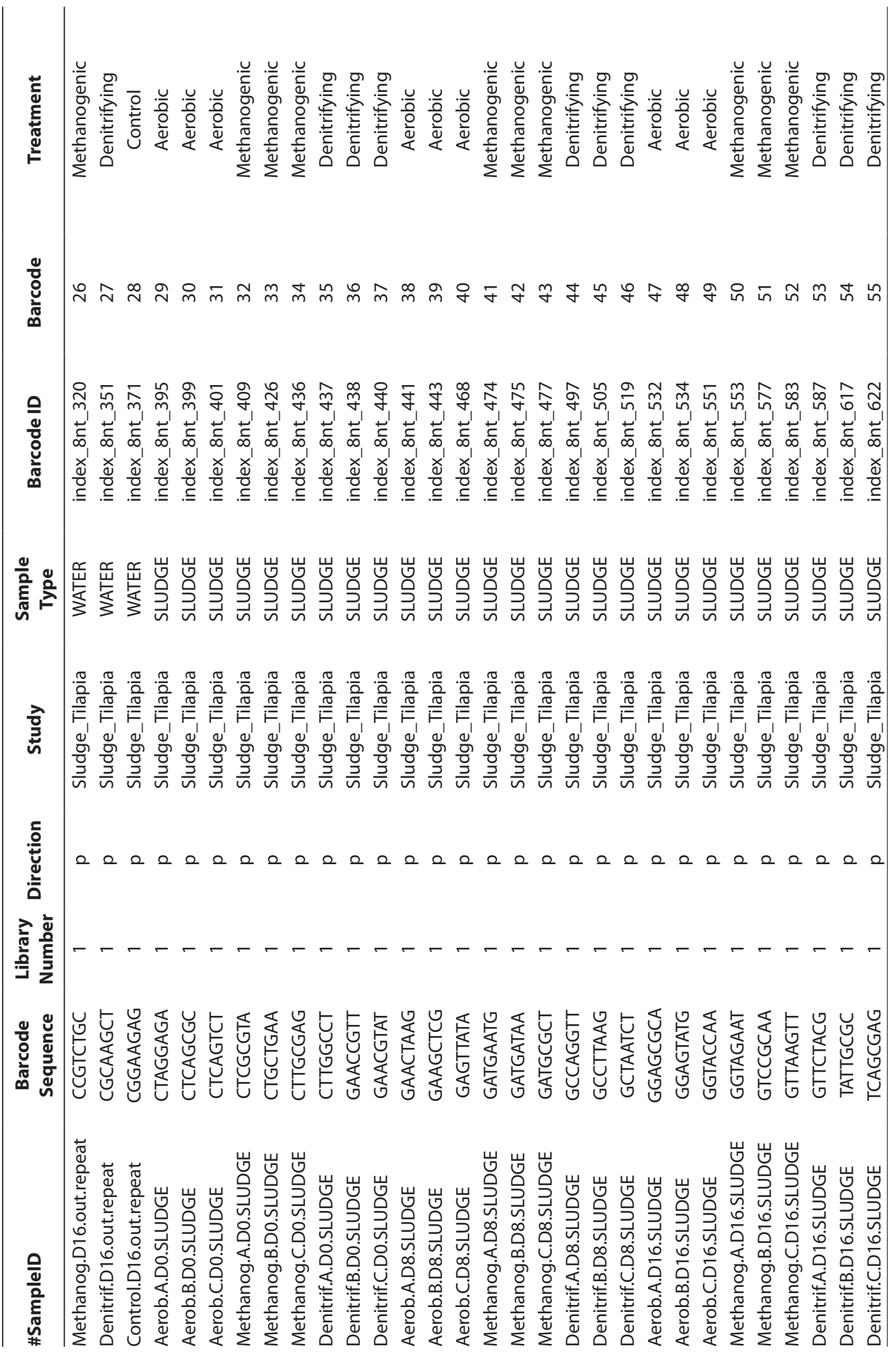




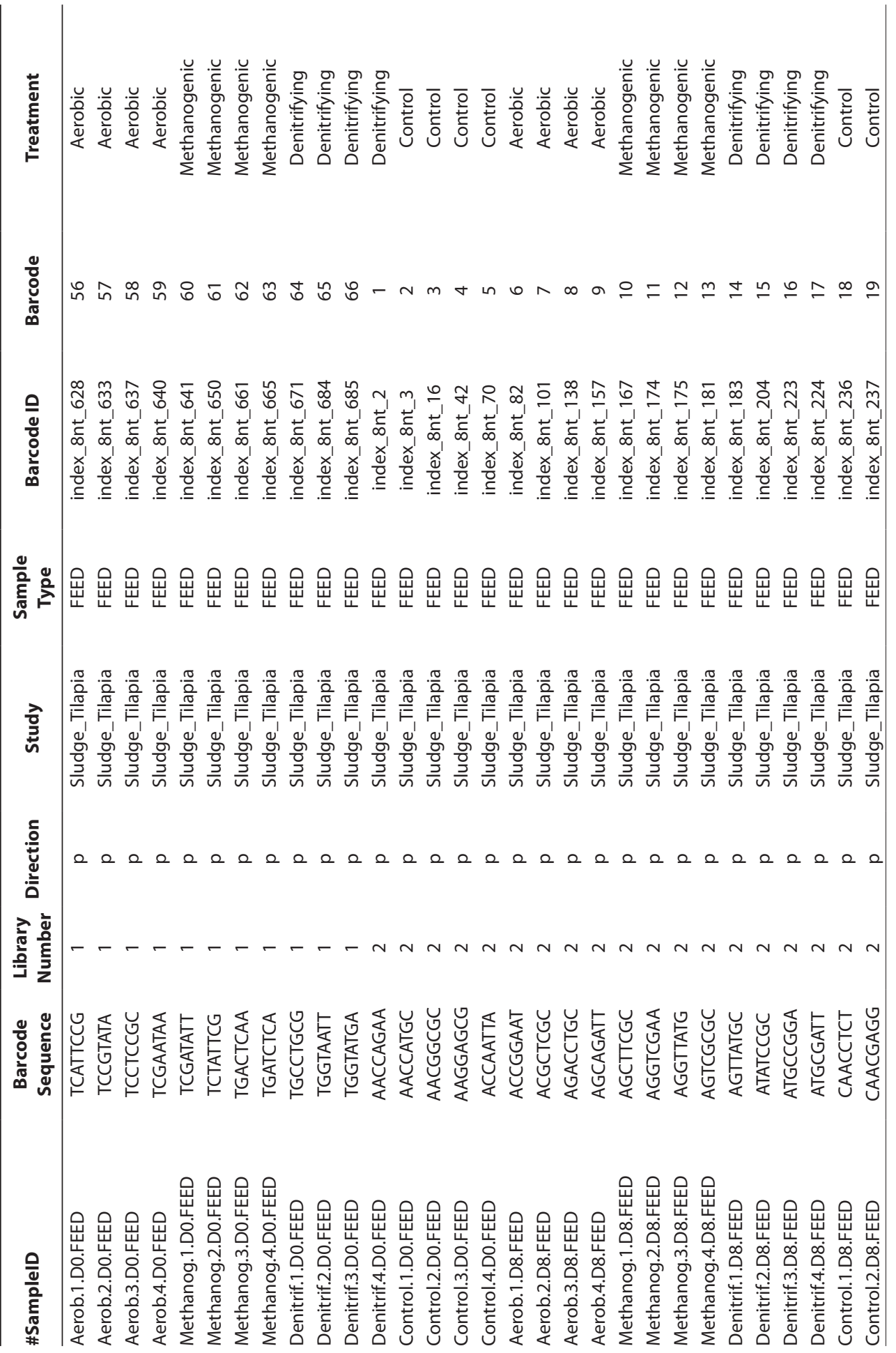




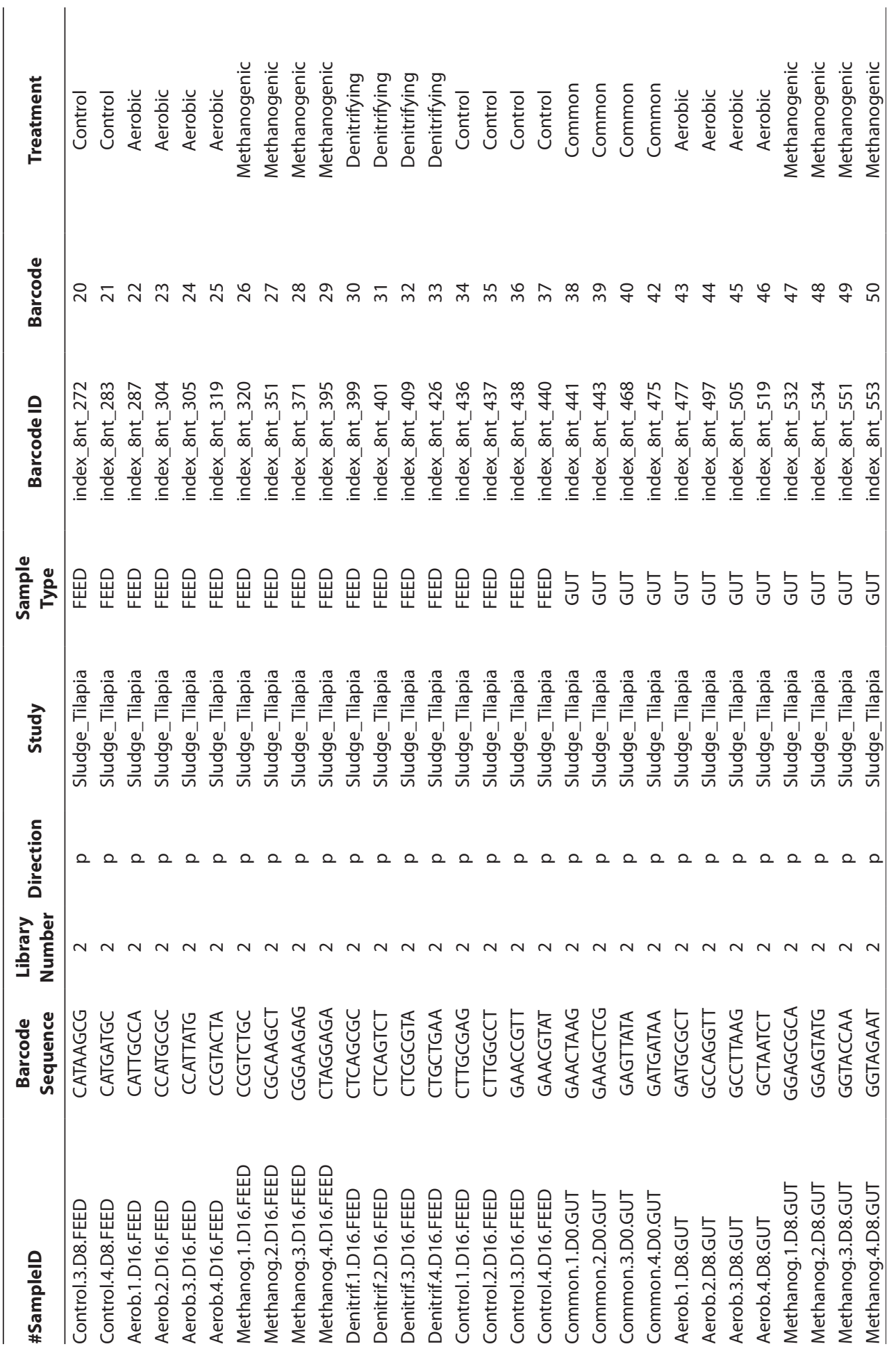




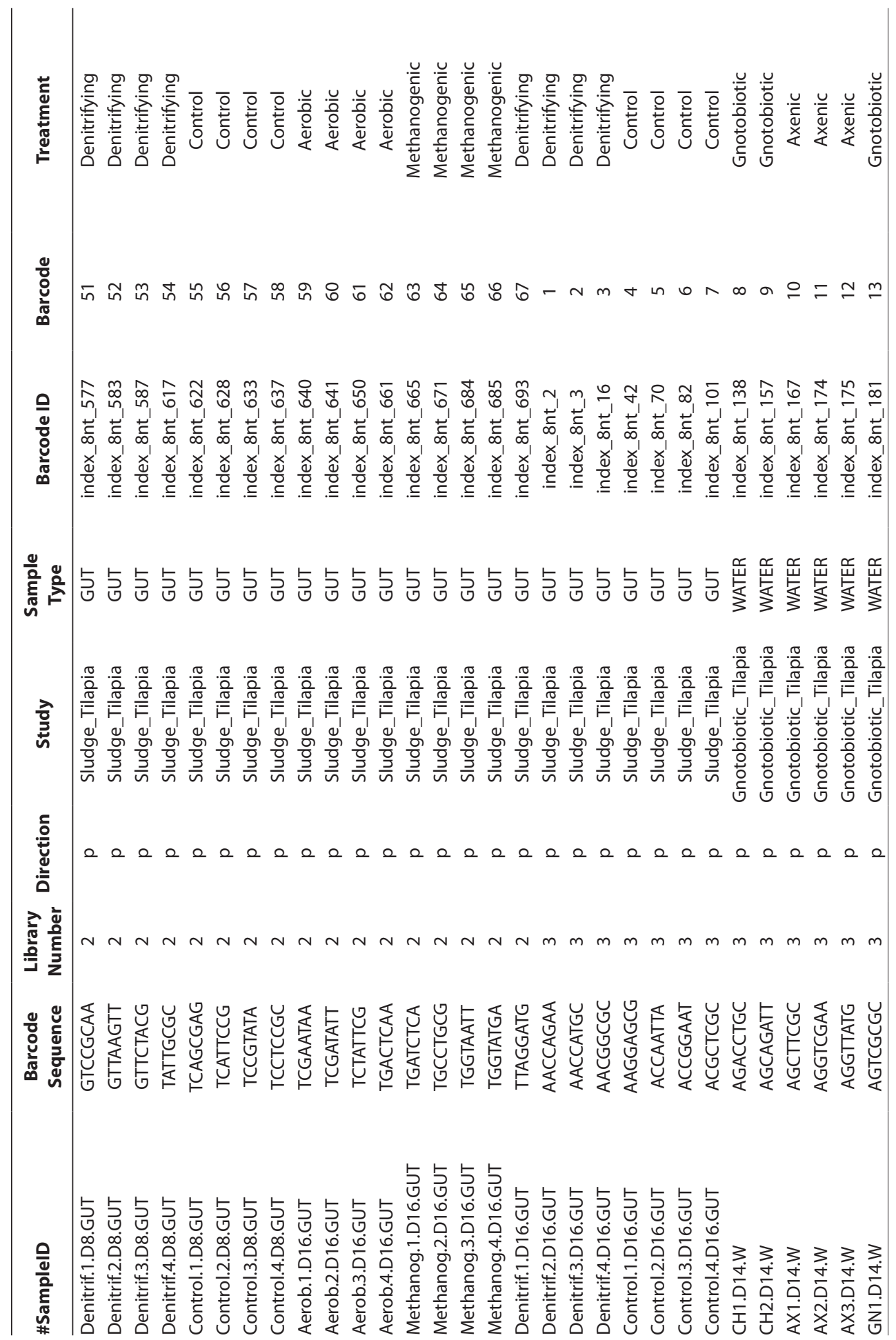




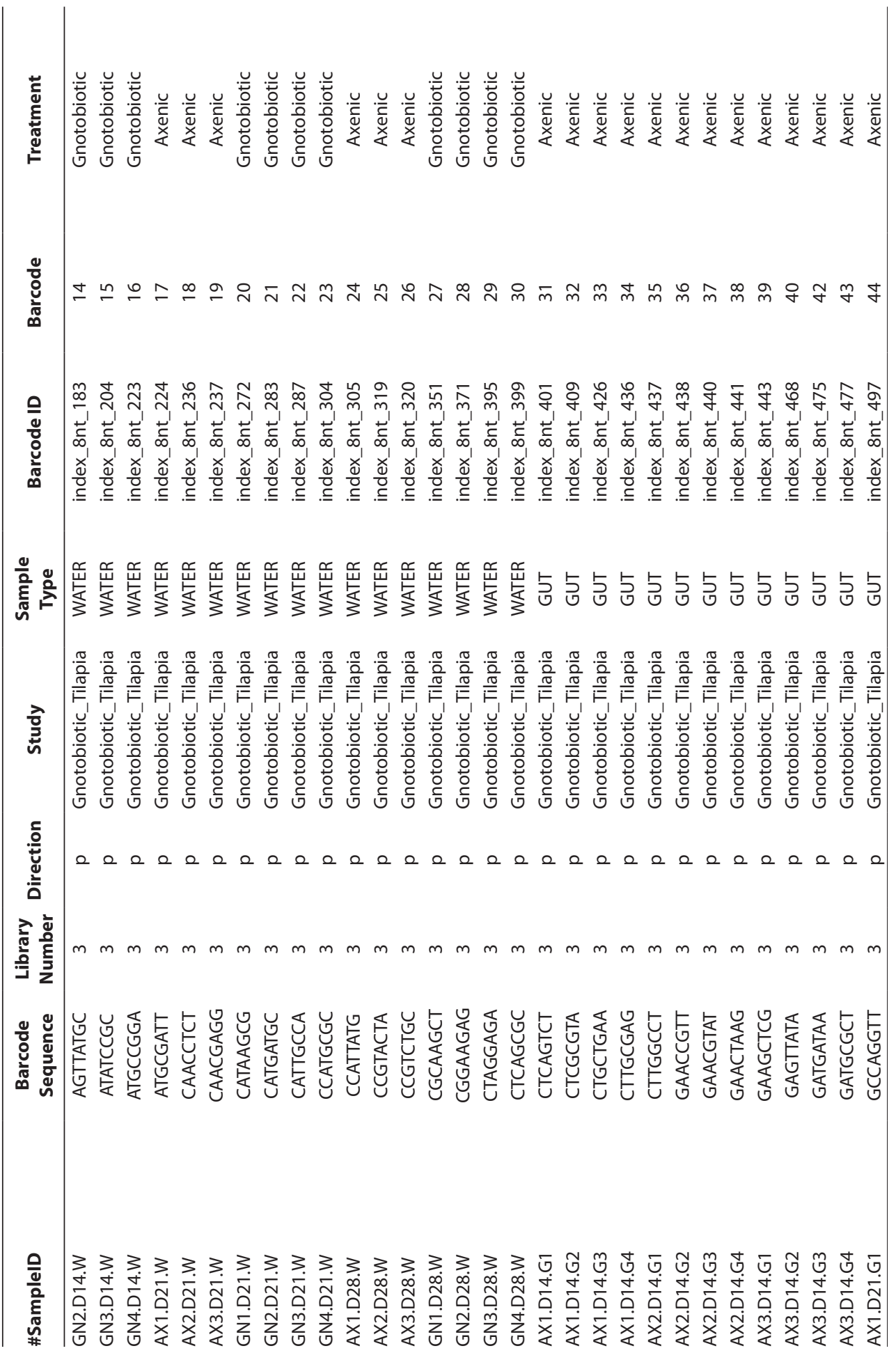




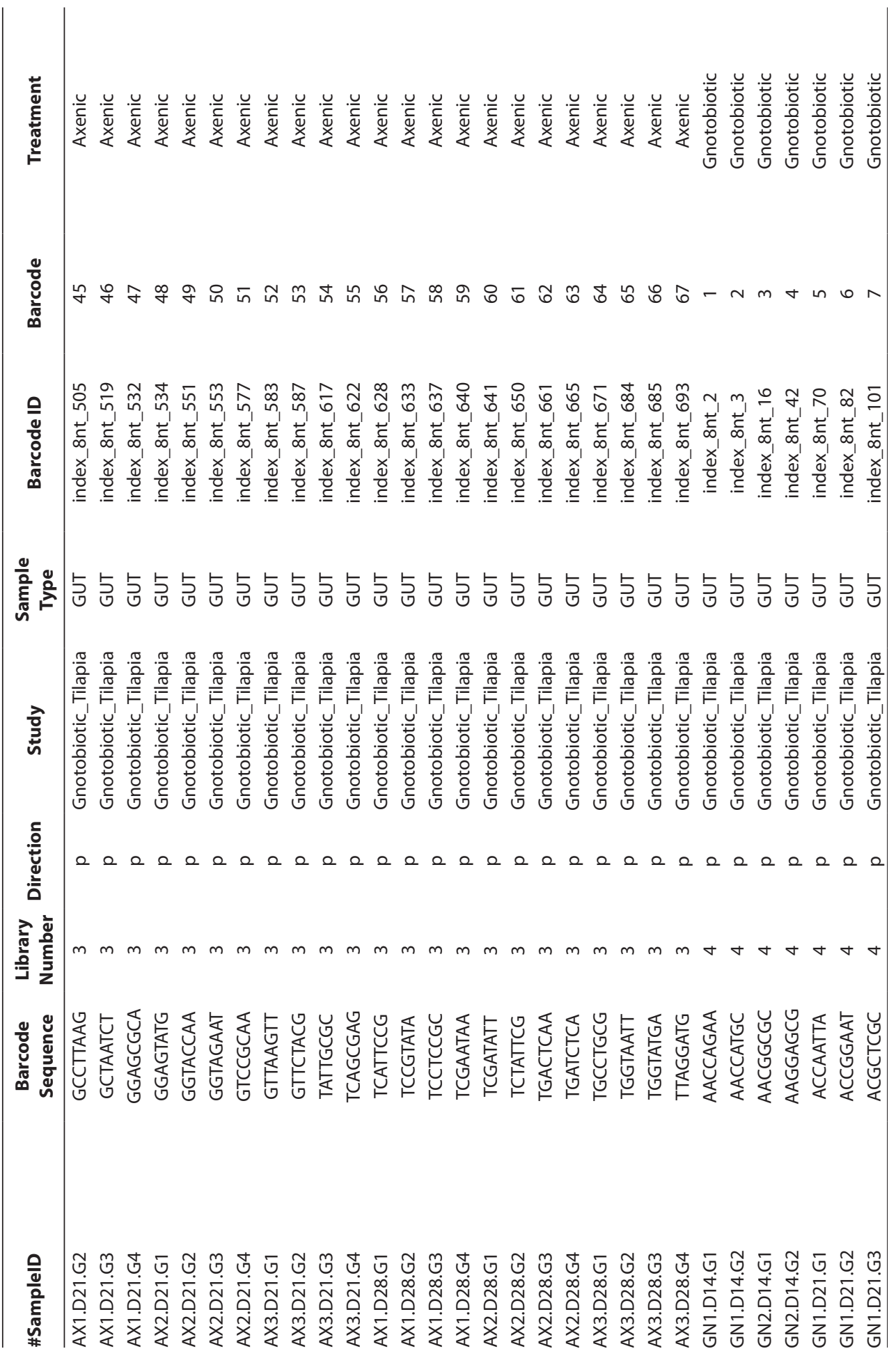




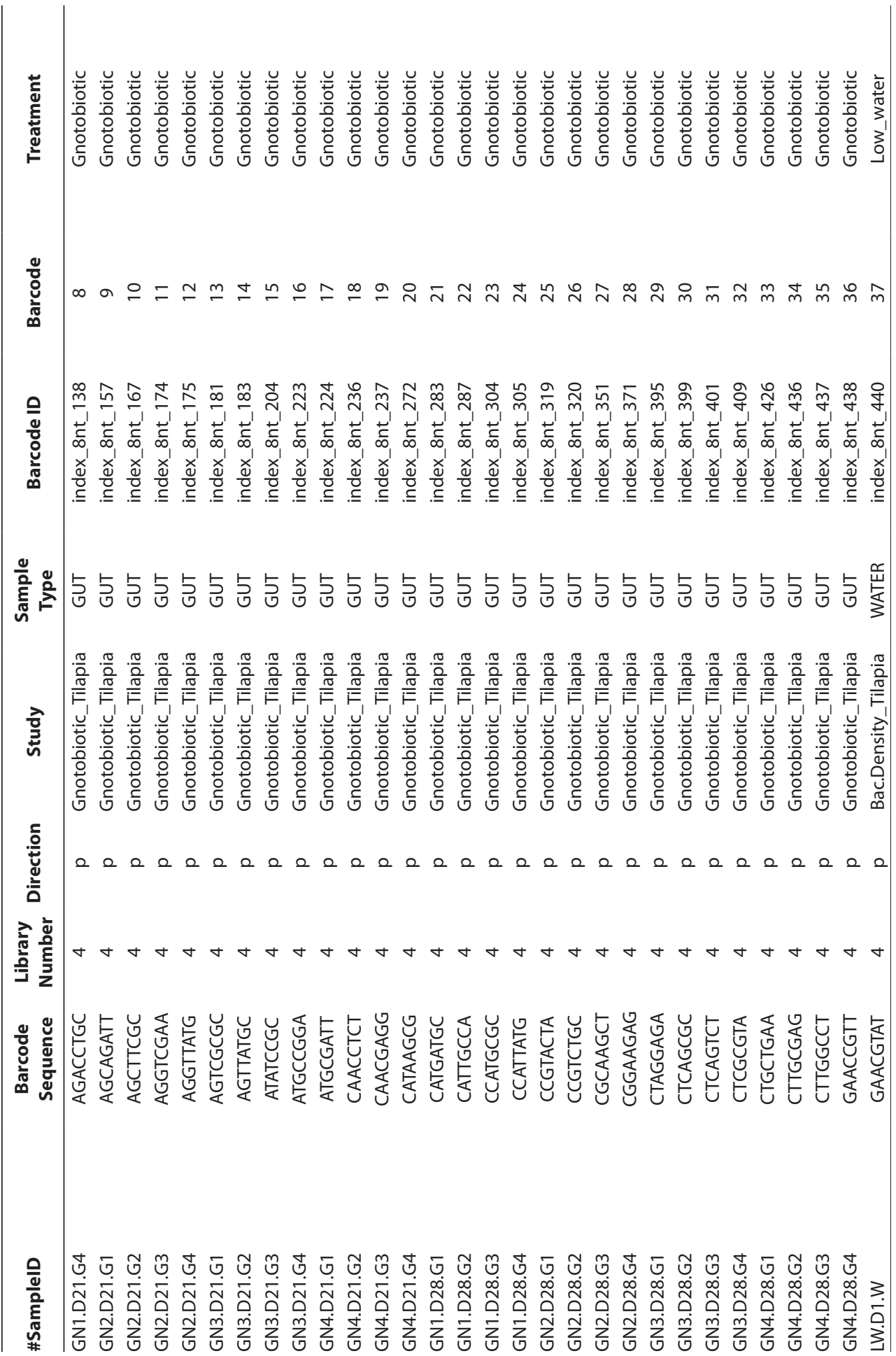




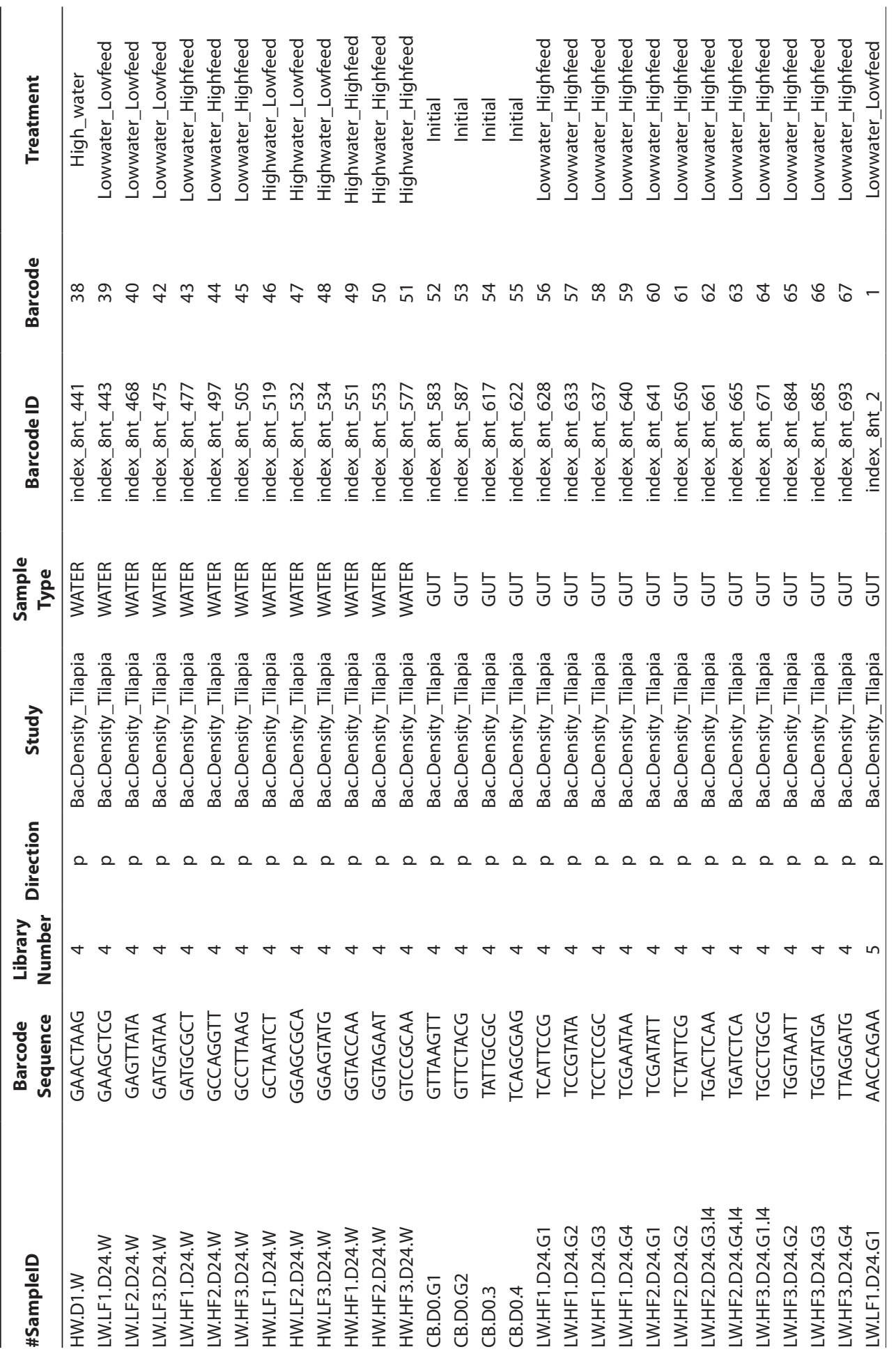




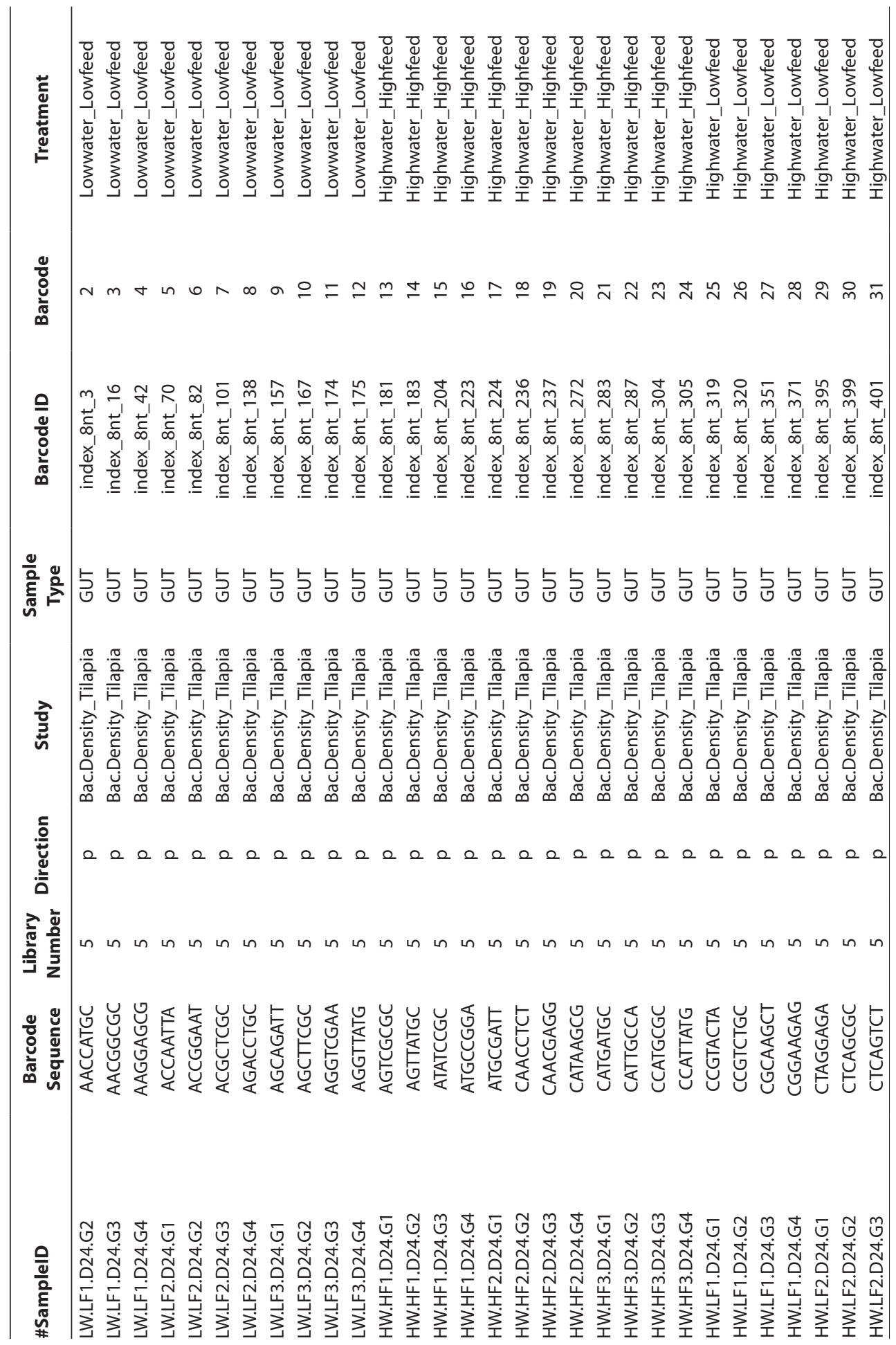




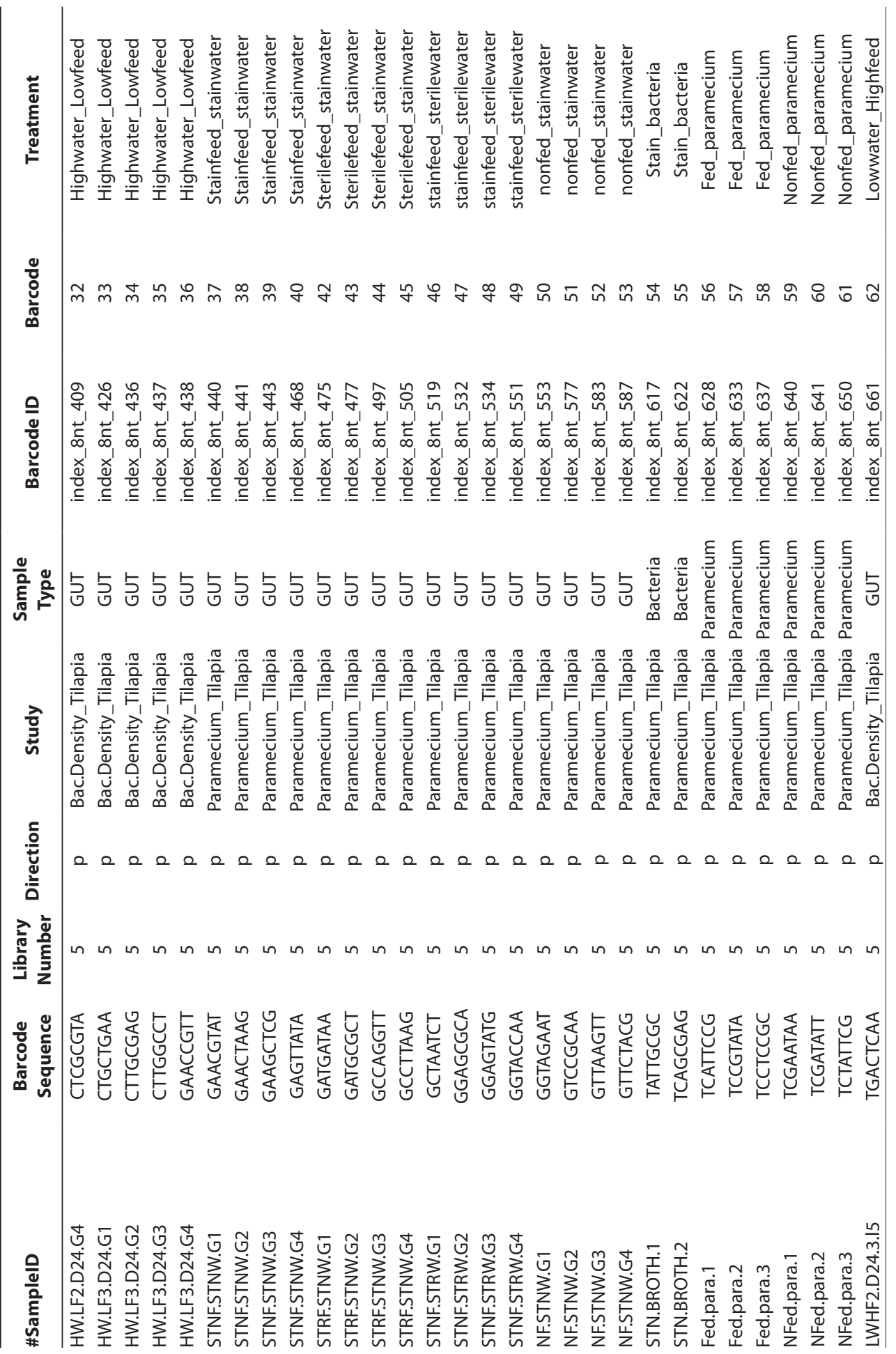




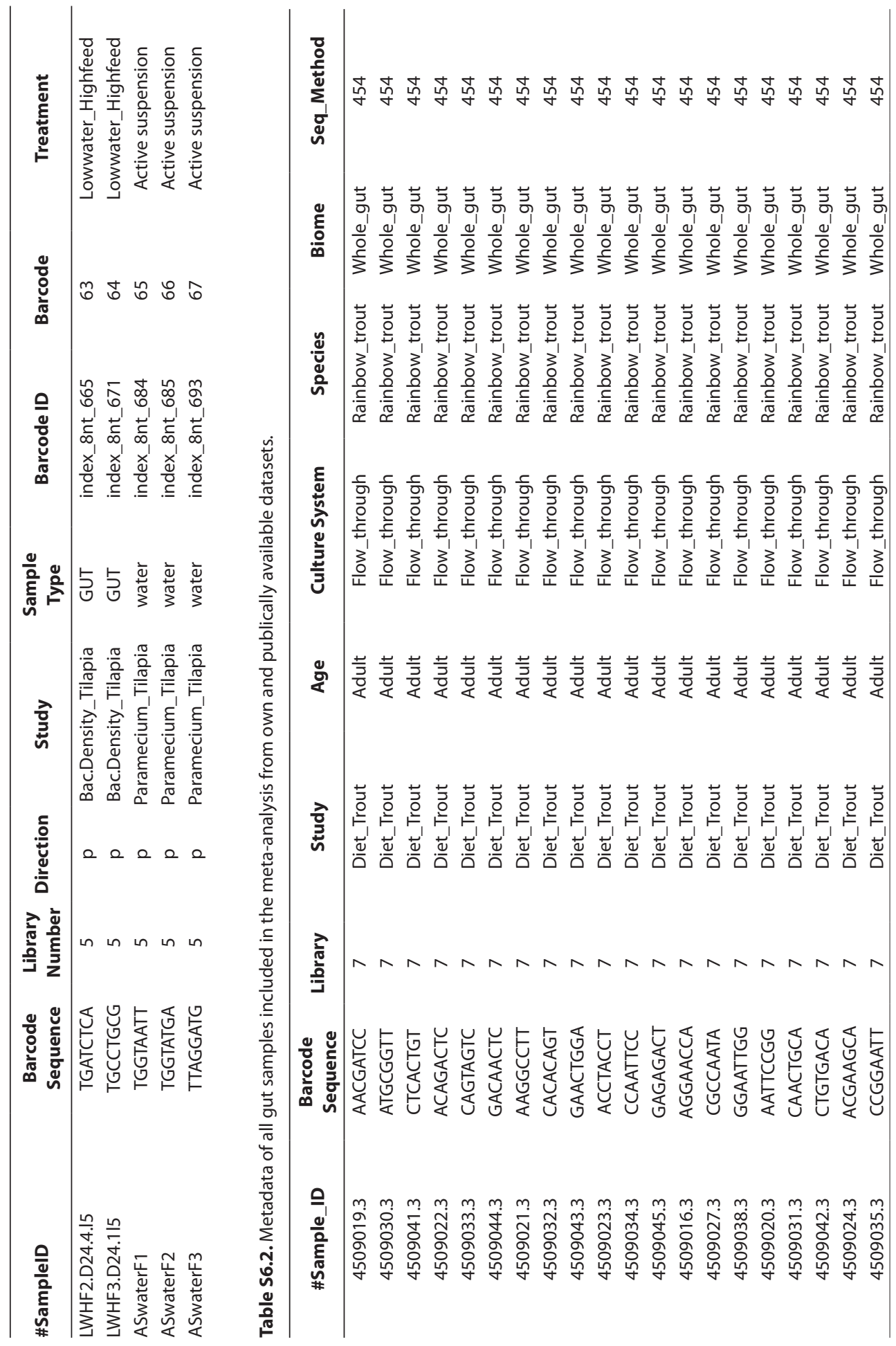




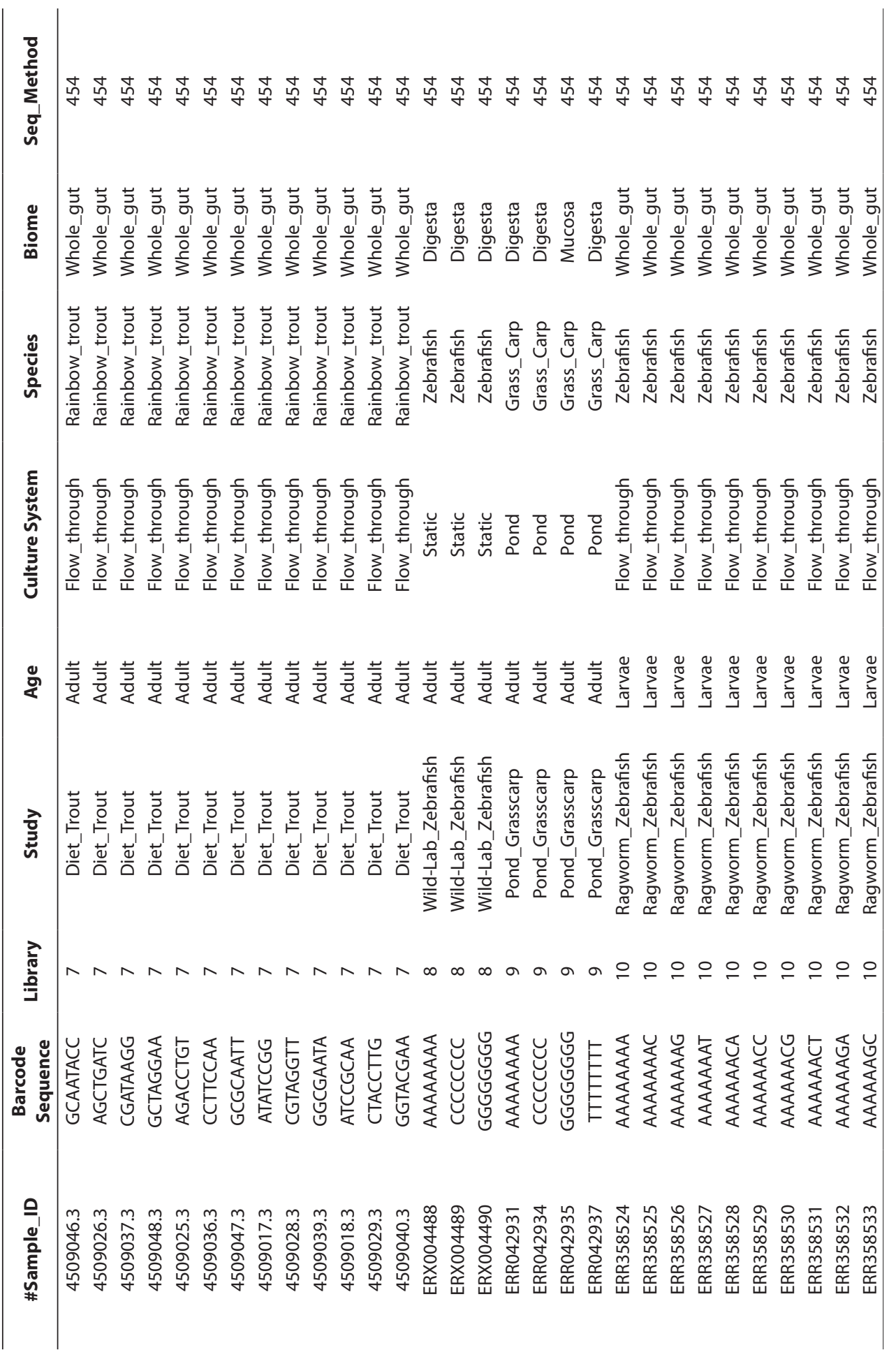




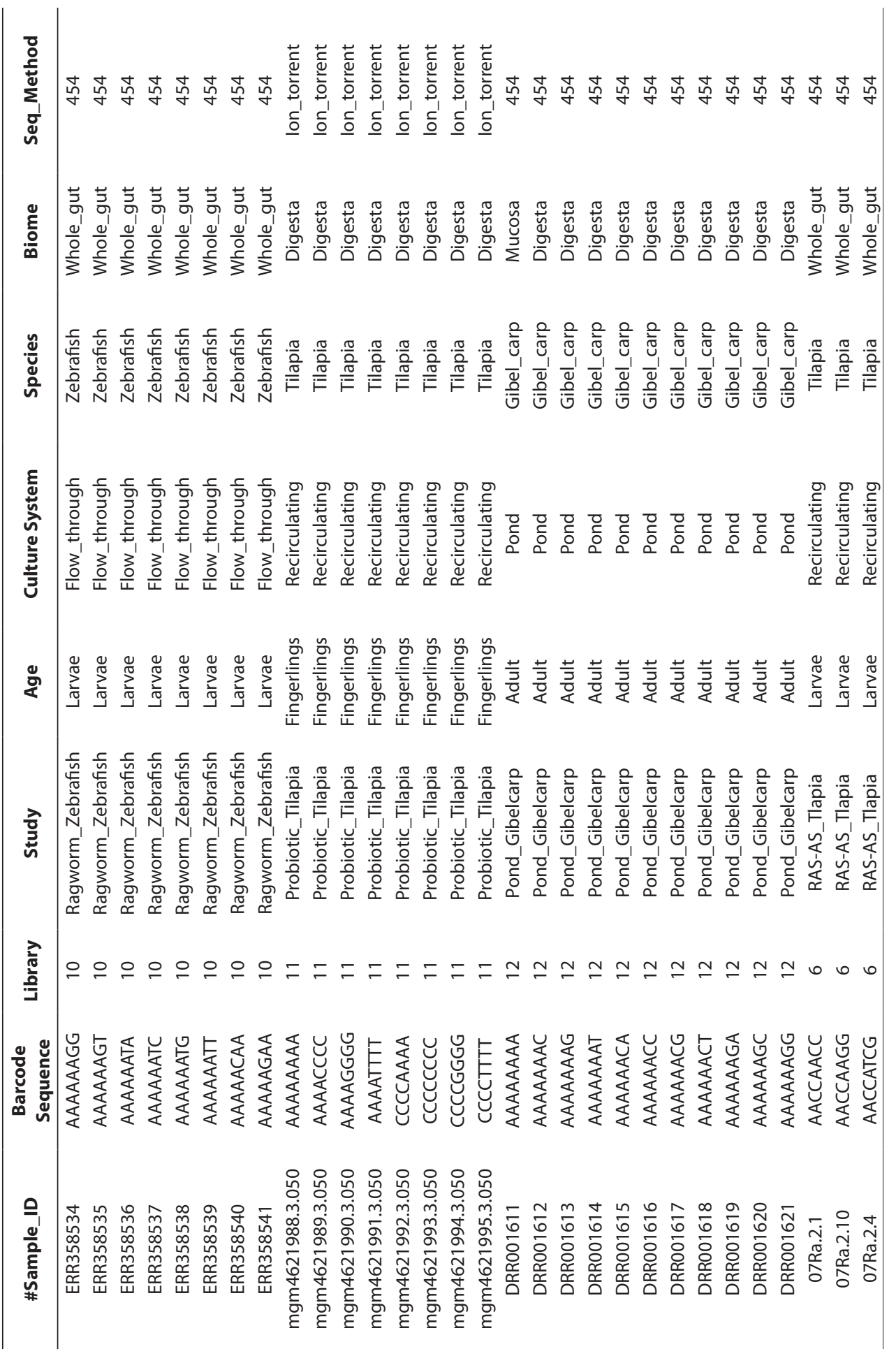




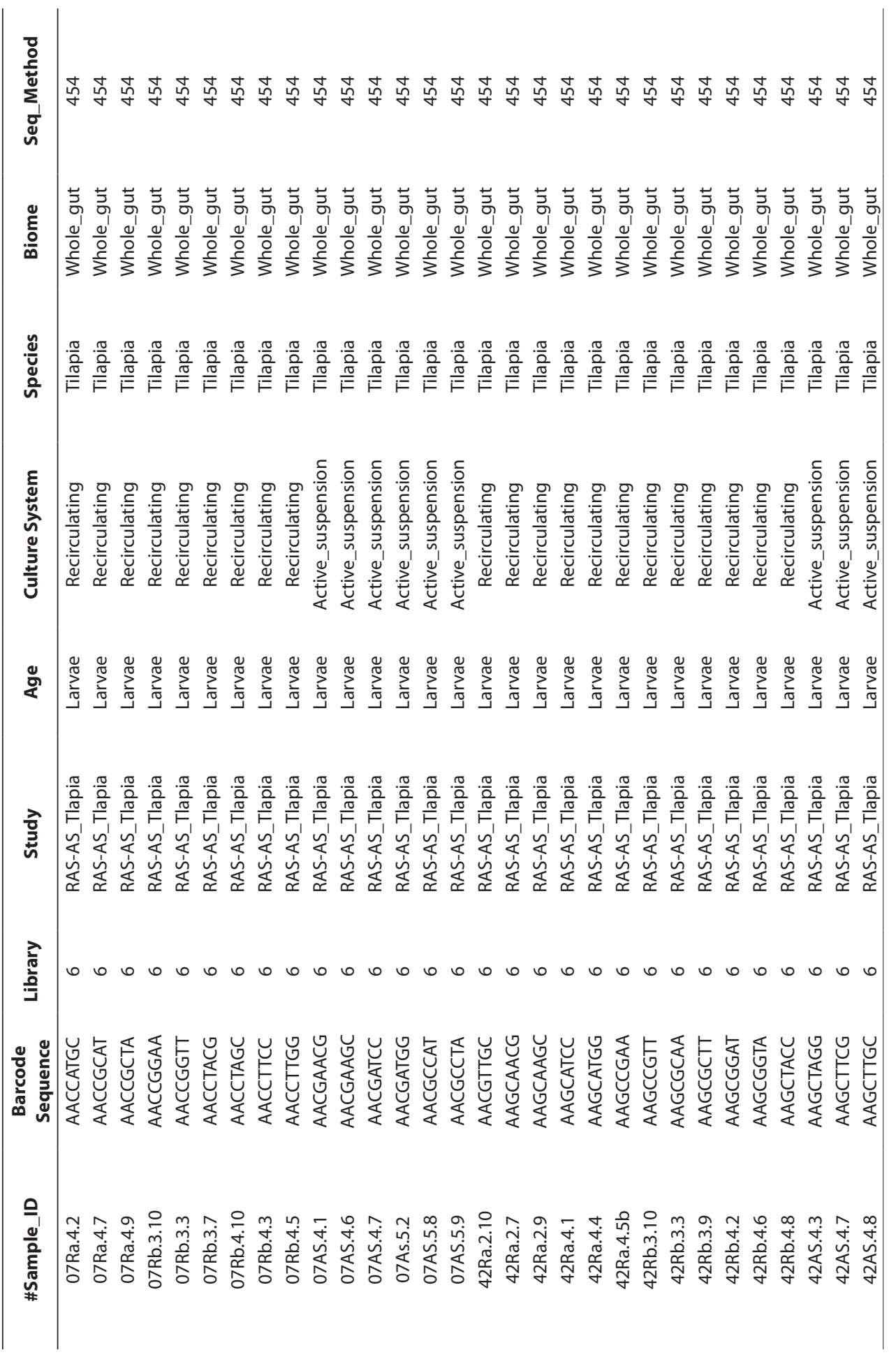




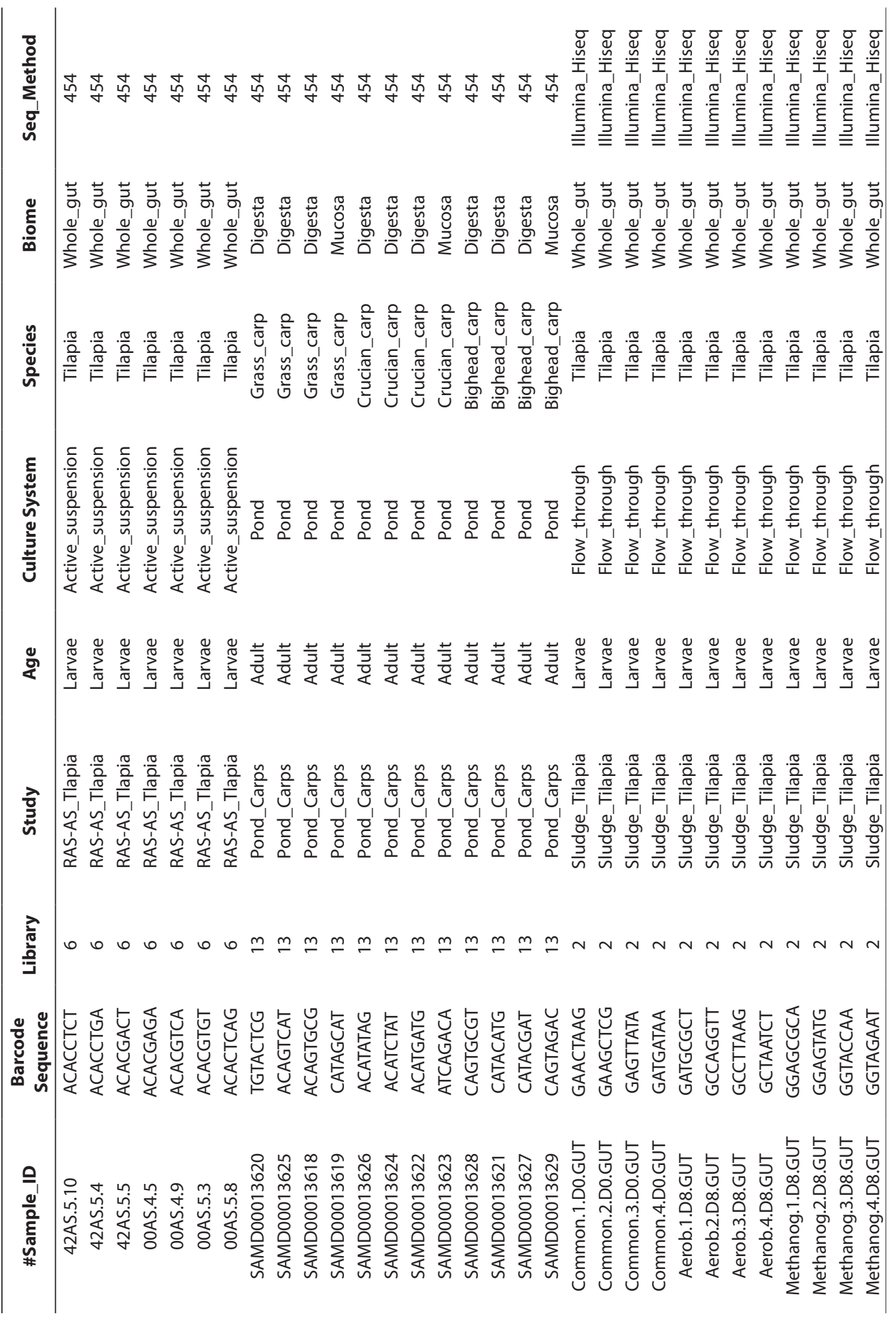




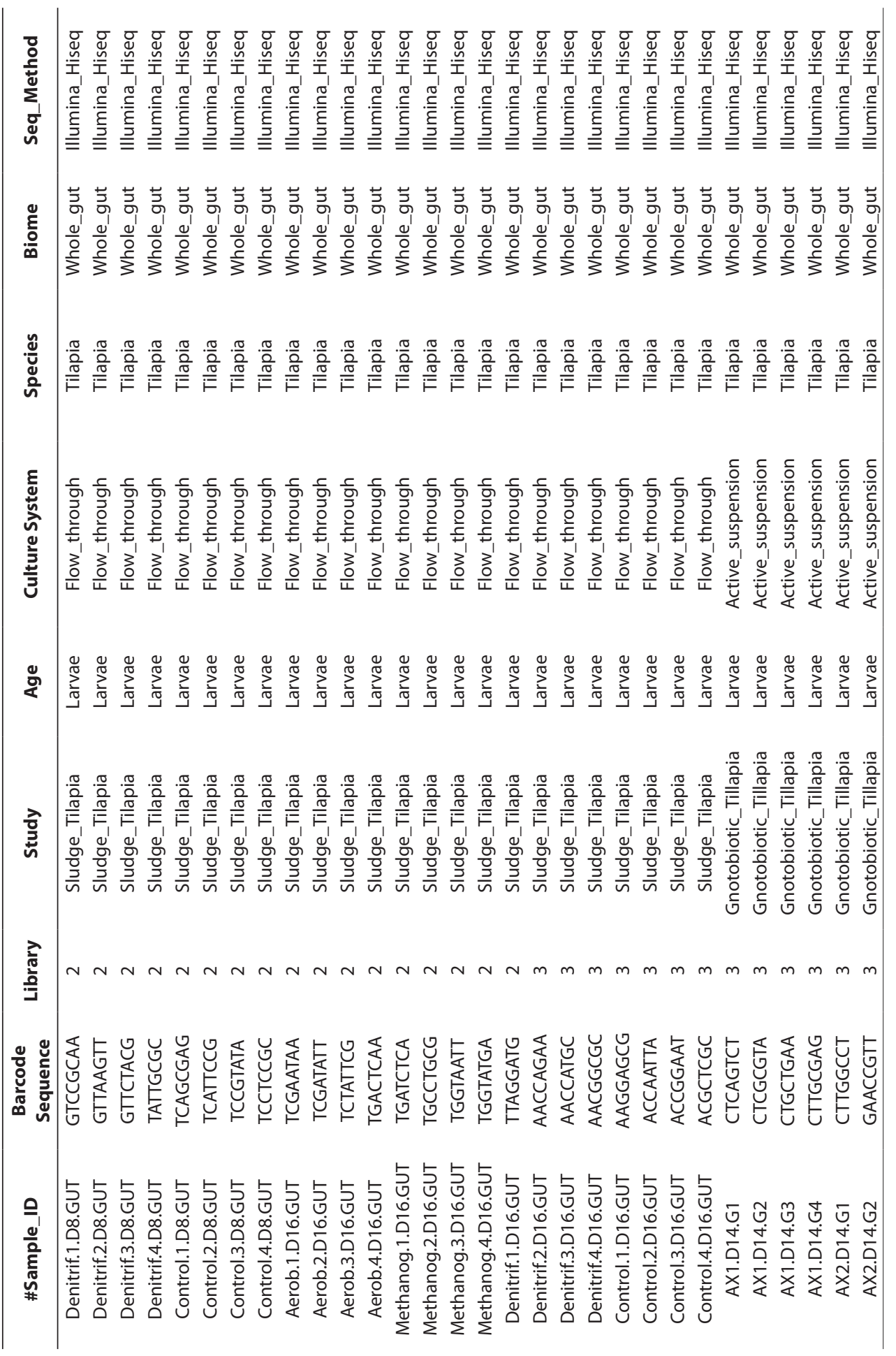









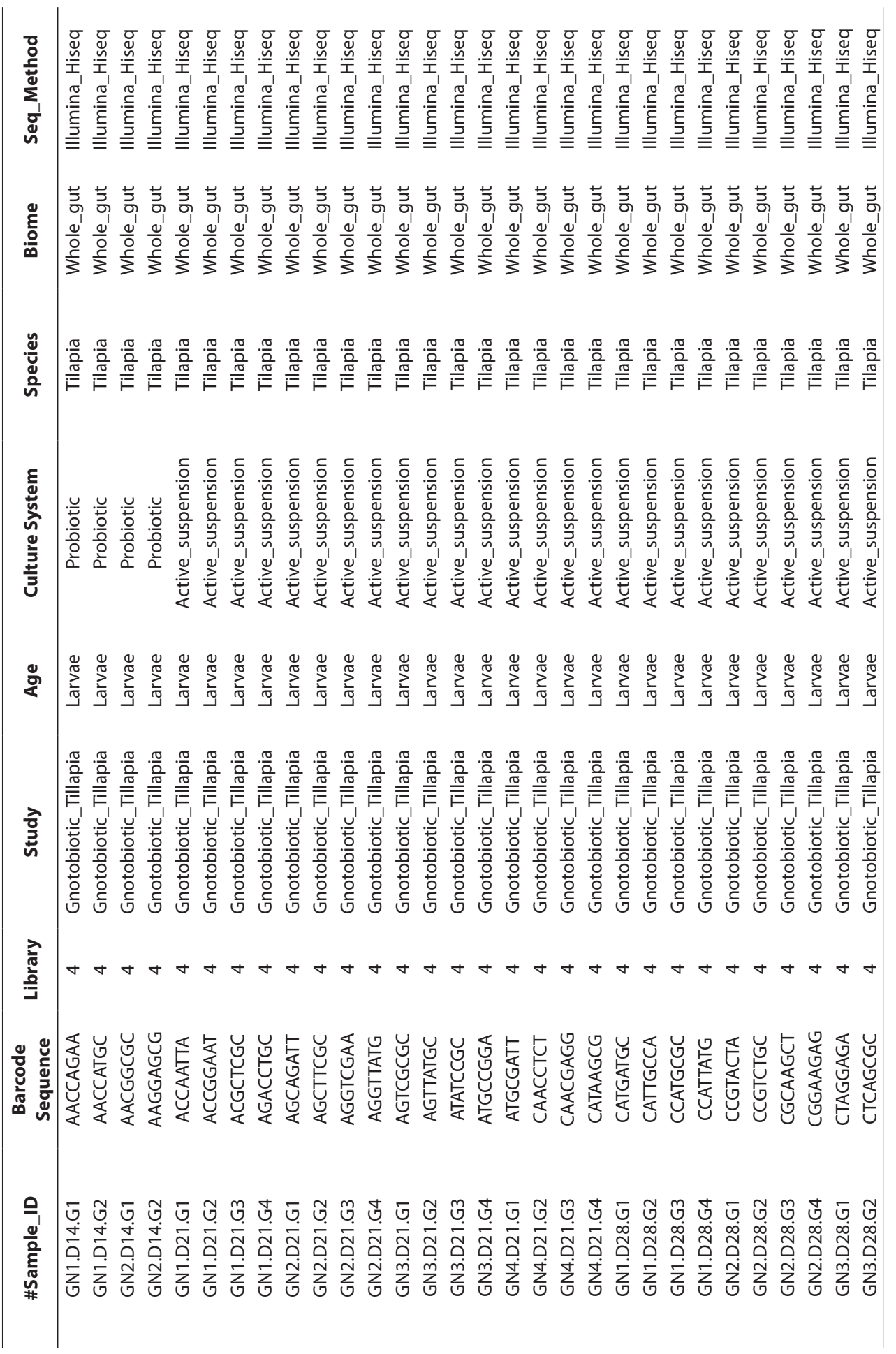




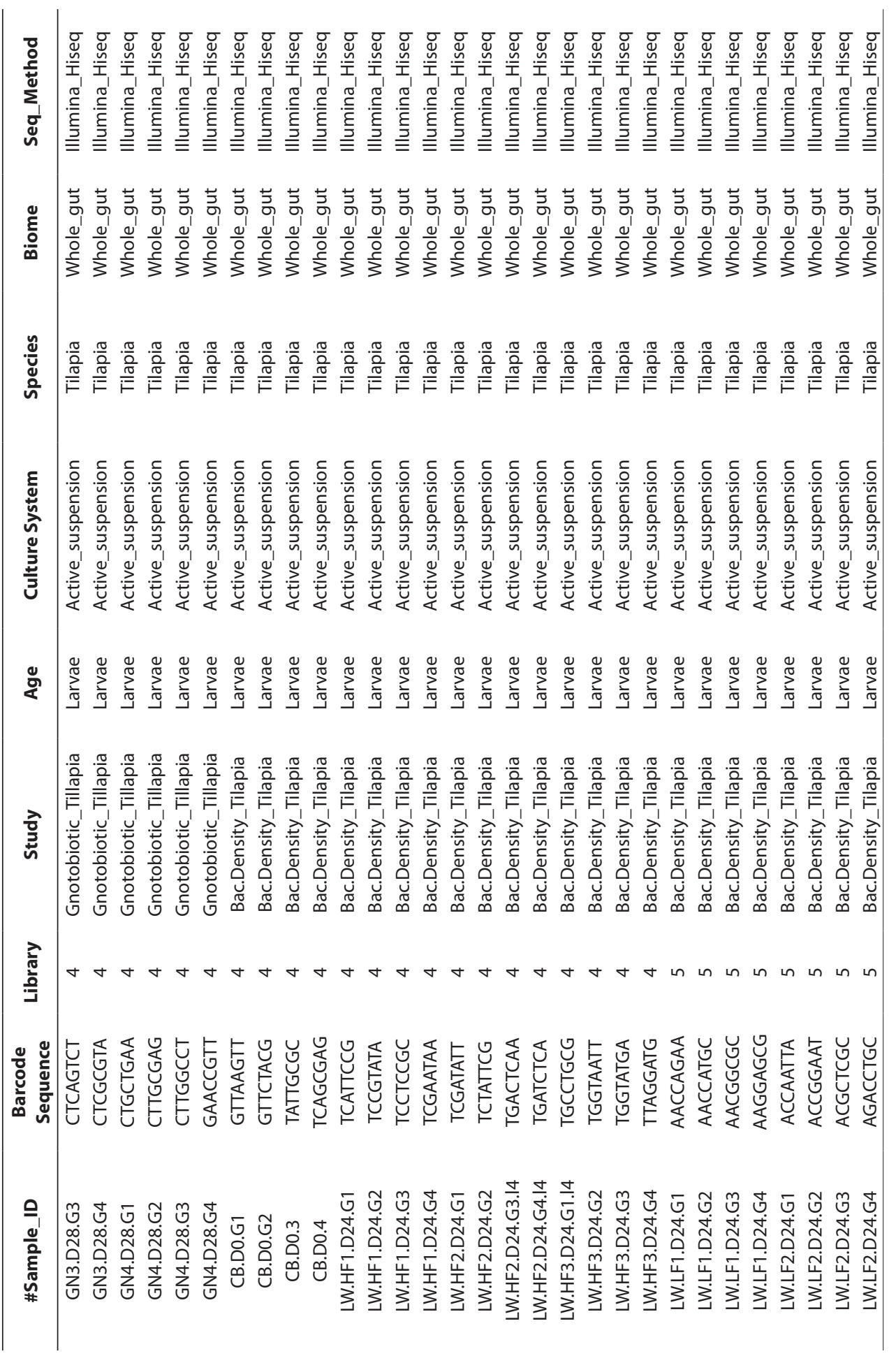




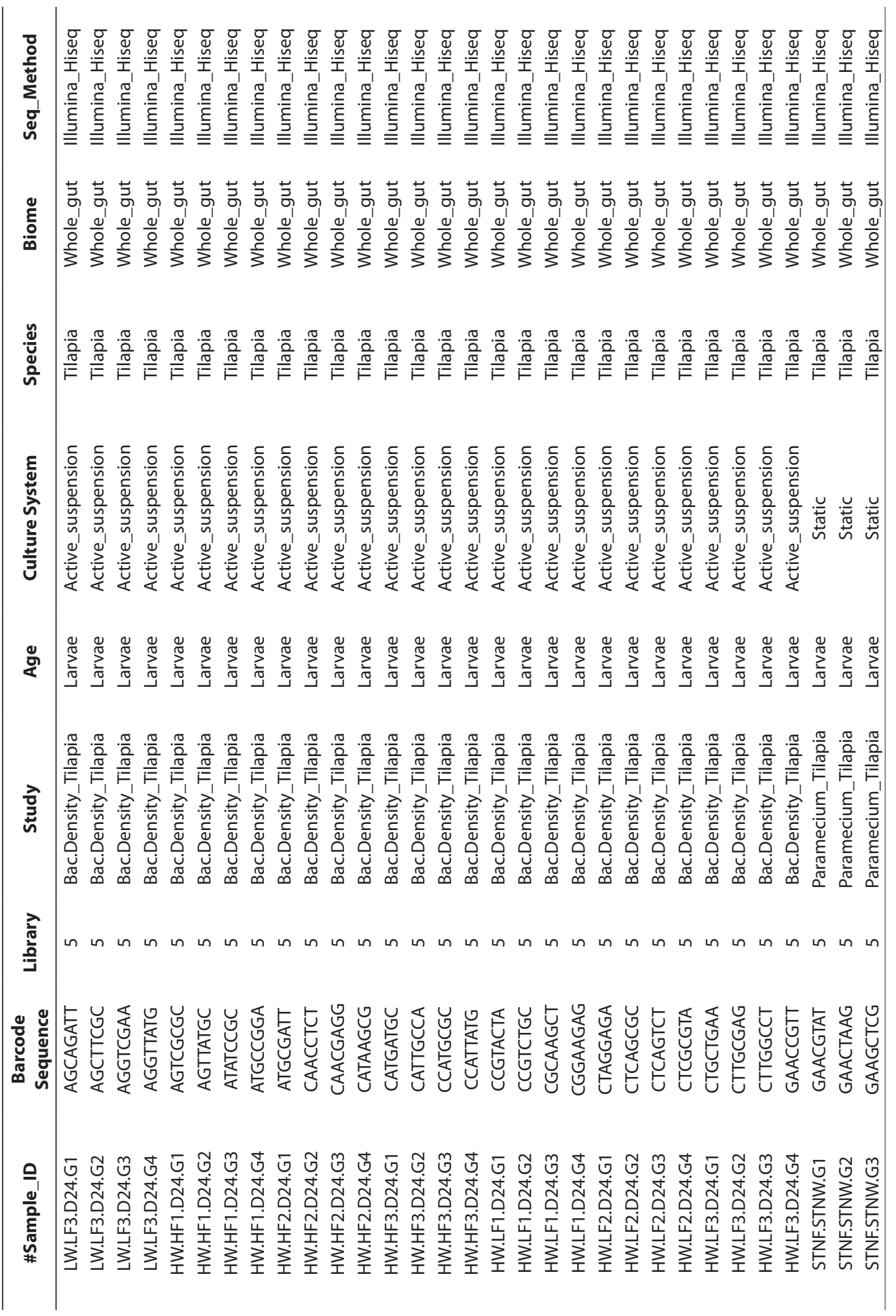







Table S6.3. References and sequence databases of the studies included in the meta-analysis

\begin{tabular}{|c|c|c|}
\hline Study & Study Reference & Sequence Database \\
\hline Diet_Trout & (Wong et al., 2013) & $\begin{array}{l}\text { http://metagenomics.anl.gov/?page=Metageno } \\
\text { meProject\&project=2870 }\end{array}$ \\
\hline Wild-Lab_Zebrafish & (Roeselers et al., 2011) & $\begin{array}{l}\text { http://www.ncbi.nlm.nih.gov/Traces/ } \\
\text { study/?acc=ERP000213 }\end{array}$ \\
\hline Pond_Grasscarp & (Wu et al., 2012b) & http://www.ebi.ac.uk/ena/data/view/ERP000842 \\
\hline Ragworm_Zebrafish & (Rurangwa et al., 2015) & $\begin{array}{l}\text { http://www.ncbi.nlm.nih.gov/Traces/ } \\
\text { study/?acc=ERP004104 }\end{array}$ \\
\hline Probiotic_Tilapia & (Standen et al., 2015) & $\begin{array}{l}\text { http://metagenomics.anl.gov/metagenomics.cgi? } \\
\text { page=MetagenomeProject\&project=12845\#jobs }\end{array}$ \\
\hline Pond_Gibelcarp & (Wu et al., 2013) & $\begin{array}{l}\text { http://www.ncbi.nlm.nih.gov/Traces/ } \\
\text { study/?acc=DRP000498 }\end{array}$ \\
\hline Pond_Carps & (Li et al., 2014a) & $\begin{array}{l}\text { http://www.ncbi.nlm.nih.gov/Traces/ } \\
\text { study/?acc=DRP001329 }\end{array}$ \\
\hline Paramecium_Tilapia & Unpublished data & NA \\
\hline Sludge_Tilapia & (Giatsis et al., 2016, under review) & SRA/NCBI accession number SRP062681 \\
\hline Gnotobiotic_Tilapia & (Giatsis et al., 2016) & SRA/NCBI accession number SRP062676 \\
\hline Bac.Density_Tilapia & Unpublished data & NA \\
\hline RAS-AS_Tilapia & (Giatsis et al., 2015) & http://www.ebi.ac.uk/ena/data/view/PRJEB4462 \\
\hline
\end{tabular}




\section{References}

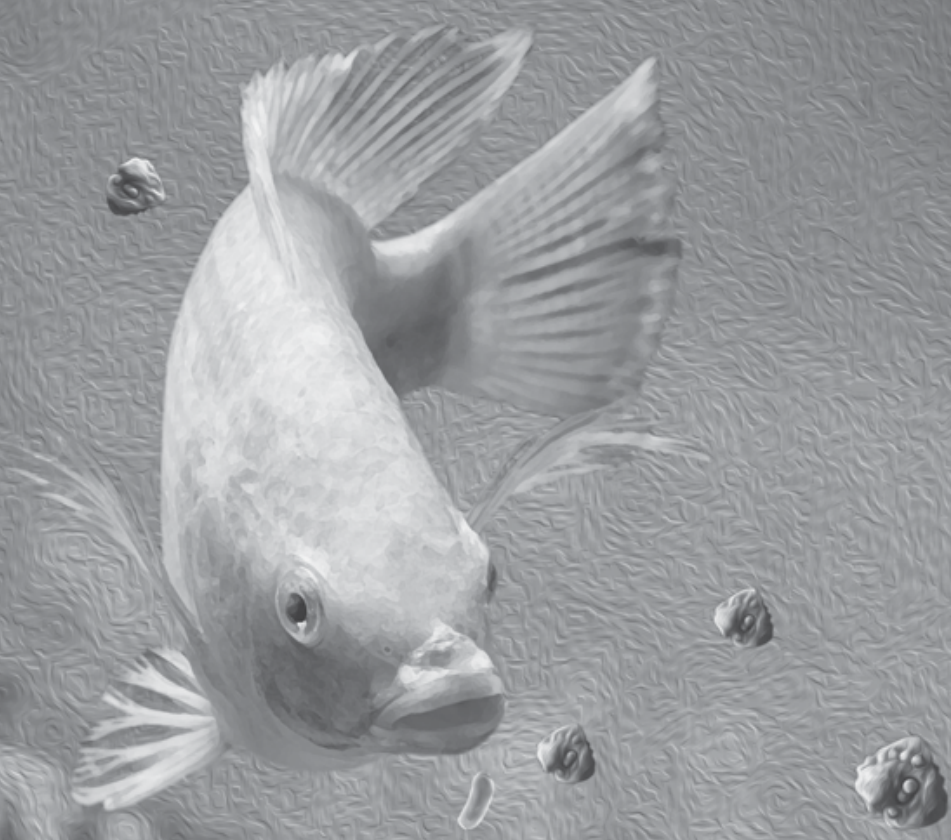

dat

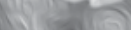

yoto

$2 \cos ^{2}$.

8

9

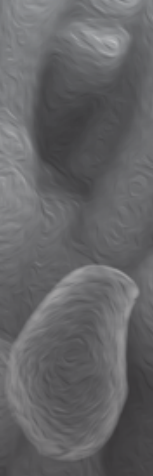

.
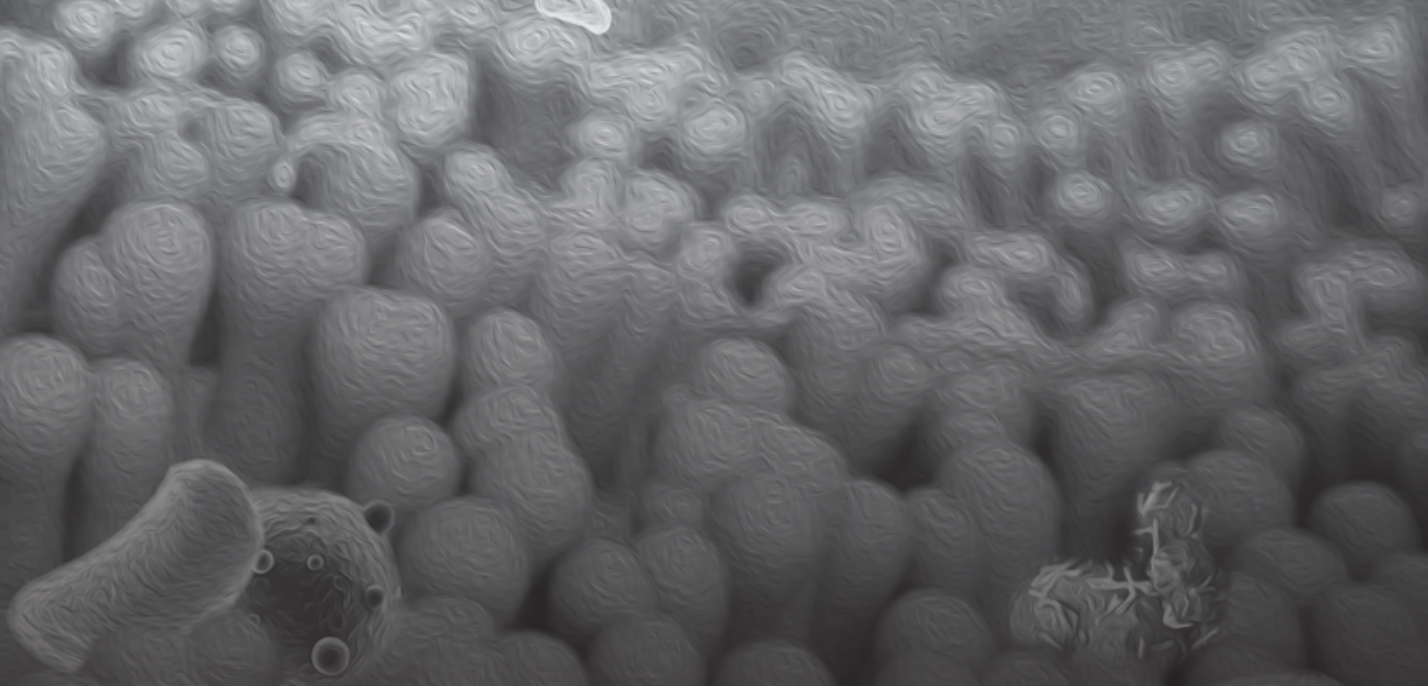

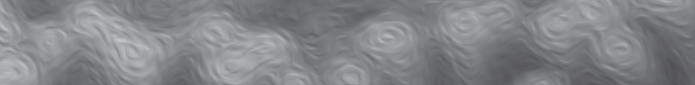





\section{References}

Adav SS, Lee D-J, Lai JY. (2010a). Microbial community of acetate utilizing denitrifiers in aerobic granules. Applied microbiology and biotechnology 85: 753-62.

Adav SS, Lee D-J, Lai J-Y. (2010b). Potential cause of aerobic granular sludge breakdown at high organic loading rates. Applied microbiology and biotechnology 85: 1601-10.

Altmann D, Stief P, Amann R, Beer D De, Schramm A. (2003). Brief report In situ distribution and activity of nitrifying bacteria in freshwater sediment. Environmental microbiology 5: 798-803.

Altschul S. (1997). Gapped BLAST and PSI-BLAST: a new generation of protein database search programs. Nucleic Acids Research 25: 3389-3402.

Aly SM, Abdel-Galil Ahmed Y, Abdel-Aziz Ghareeb A, Mohamed MF. (2008). Studies on Bacillus subtilis and Lactobacillus acidophilus, as potential probiotics, on the immune response and resistance of Tilapia nilotica (Oreochromis niloticus) to challenge infections. Fish \& shellfish immunology 25: 128-36.

Amato L, Ritschard JS, Kurtz O, Arias-Roth E, Lacroix C, Schuppler M, Meile L. (2012). Microbial composition of defect smear - A problem evolving during foil-prepacked storage of red-smear cheeses. International Dairy Journal 27: 77-85.

Anderson MJ. (2001). A new method for non-parametric multivariate analysis of variance. Austral Ecology 26: 32-46.

Anderson MJ. (2006). Distance-based tests for homogeneity of multivariate dispersions. Biometrics 62: 245-253.

Anderson MJ, Ellingsen KE, McArdle BH. (2006). Multivariate dispersion as a measure of beta diversity. Ecology Letters 9: 683-693.

Anderson MJ, Robinson J. (2003). Generalized discriminant analysis based on distances. Aust NZJ Stat 45: $301-318$. Angelakis E, Armougom F, Million M, Raoult D. (2012). The relationship between gut microbiota and weight gain in humans. Future microbiology 7: 91-109.

Arenskötter M, Bröker D, Steinbüchel A. (2004). Biology of the metabolically diverse genus Gordonia. Applied and environmental microbiology 70: 3195-204.

Arias CR, Koenders K, Larsen AM. (2013). Predominant bacteria associated with red snapper from the Northern Gulf of Mexico. Journal of aquatic animal health 25: 281-9.

Askarian F, Zhou Z, Olsen RE, Sperstad S, Ringø E. (2012). Culturable autochthonous gut bacteria in Atlantic salmon (Salmo salar L.) fed diets with or without chitin. Characterization by $16 \mathrm{~S}$ rRNA gene sequencing, ability to produce enzymes and in vitro growth inhibition of four fish pathogens. Aquaculture 326-329: 1-8.

Attramadal KJKK, Salvesen I, Xue R, Øie G, Størseth TR, Vadstein O, Olsen Y. (2012). Recirculation as a possible microbial control strategy in the production of marine larvae. Aquacultural Engineering 46: 27-39.

Auffret M, Yergeau É, Pilote A, Proulx É, Proulx D, Greer CW, Vandenberg G, Villemur R. (2013). Impact of water quality on the bacterial populations and off-flavours in recirculating aquaculture systems. FEMS Microbiology Ecology 84: 235-247.

Austin B. (1983). Bacterial microflora associated with a coastal, marine fish-rearing unit. Journal of the Marine Biological Association of the United Kingdom 63: 585.

Austin B. (2006). The bacterial microflora of fish, revised. TheScientificWorldJournal 6: 931-45.

Avnimelech Y. (2007). Feeding with microbial flocs by tilapia in minimal discharge bio-flocs technology ponds. Aquaculture 264: 140-147.

Azim ME, Little DC. (2008). The biofloc technology (BFT) in indoor tanks: Water quality, biofloc composition, and growth and welfare of Nile tilapia (Oreochromis niloticus). Aquaculture 283: 29-35. 
Bakke I, Skjermo J, Vo TA, Vadstein O. (2013). Live feed is not a major determinant of the microbiota associated with cod larvae (Gadus morhua). Environmental Microbiology Reports 5: 537-548.

Bakke-McKellep AM, Penn MH, Salas PM, Refstie S, Sperstad S, Landsverk T, Ringø E, Krogdahl A. (2007). Effects of dietary soyabean meal, inulin and oxytetracycline on intestinal microbiota and epithelial cell stress, apoptosis and proliferation in the teleost Atlantic salmon (Salmo salar L.). The British journal of nutrition 97: 699-713.

Balcázar JL, de Blas I, Ruiz-Zarzuela I, Vendrell D, Gironés O, Muzquiz JL. (2007). Sequencing of variable regions of the 16S rRNA gene for identification of lactic acid bacteria isolated from the intestinal microbiota of healthy salmonids. Comparative immunology, microbiology and infectious diseases 30: 111-8.

Balcázar JL, Rojas-Luna T. (2007). Inhibitory activity of probiotic Bacillus subtilis UTM 126 against vibrio species confers protection against vibriosis in juvenile shrimp (Litopenaeus vannamei). Current microbiology $\mathbf{5 5}$ 409-12.

Baxter NT, Wan JJ, Schubert AM, Jenior ML, Myers P, Schloss PD. (2015). Intra- and Interindividual Variations Mask Interspecies Variation in the Microbiota of Sympatric Peromyscus Populations. Applied and Environmental Microbiology 81: 396-404.

Bell T, Newman JA, Silverman BW, Turner SL, Lilley AK. (2005). The contribution of species richness and composition to bacterial services. Nature 436: 1157-60.

Benson AK, Kelly S a, Legge R, Ma F, Low SJ, Kim J, Zhang M, Oh PL, Nehrenberg D, Hua K, Kachman SD, Moriyama EN, Walter J, Peterson D a, Pomp D. (2010). Individuality in gut microbiota composition is a complex polygenic trait shaped by multiple environmental and host genetic factors. Proceedings of the National Academy of Sciences of the United States of America 107: 18933-18938.

Besemer K, Peter H, Logue JB, Langenheder S, Lindström ES, Tranvik LJ, Battin TJ. (2012). Unraveling assembly of stream biofilm communities. The ISME journal 6: 1459-68.

van den Bogert B, Erkus O, Boekhorst J, de Goffau M, Smid EJ, Zoetendal EG, Kleerebezem M. (2013). Diversity of human small intestinal Streptococcus and Veillonella populations. FEMS microbiology ecology 85: 376-88.

Van den Bogert B, de Vos WM, Zoetendal EG, Kleerebezem M. (2011). Microarray analysis and barcoded pyrosequencing provide consistent microbial profiles depending on the source of human intestinal samples. Applied and environmental microbiology 77: 2071-80.

Bolnick DI, Snowberg LK, Hirsch PE, Lauber CL, Org E, Parks B, Lusis AJ, Knight R, Caporaso JG, Svanbäck R. (2014). Individual diet has sex-dependent effects on vertebrate gut microbiota. Nature communications 5: 4500 .

Boscaro V, Felletti M, Vannini C, Ackerman MS, Chain PSG, Malfatti S, Vergez LM, Shin M, Doak TG, Lynch M, Petroni G. (2013). Polynucleobacter necessarius, a model for genome reduction in both free-living and symbiotic bacteria. Proceedings of the National Academy of Sciences of the United States of America 110: 18590-5.

Boutin S, Audet C, Derome N. (2013a). Probiotic treatment by indigenous bacteria decreases mortality without disturbing the natural microbiota of Salvelinus fontinalis. Canadian journal of microbiology 59: 662-70.

Boutin S, Bernatchez L, Audet C, Derôme N. (2013b). Network analysis highlights complex interactions between pathogen, host and commensal microbiota. PloS one 8: e84772.

Boutin S, Sauvage C, Bernatchez L, Audet C, Derome N. (2014). Inter individual variations of the fish skin microbiota: Host genetics basis of mutualism? PLOS ONE 9: e102649.

Bowden TJ, Cook P, Rombout JHWM. (2005). Development and function of the thymus in teleosts. Fish \& shellfish immunology 19: 413-27.

Bräuer SL, Adams C, Kranzler K, Murphy D, Xu M, Zuber P, Simon HM, Baptista a M, Tebo BM. (2011). Culturable Rhodobacter and Shewanella species are abundant in estuarine turbidity maxima of the Columbia River. Environmental microbiology 13: 589-603.

Bray JR, Curtis JT. (1957). An Ordination of the Upland Forest Communities of Southern Wisconsin. Ecological Monographs 27: 325.

Brito LO, Arantes R, Magnotti C, Derner R, Pchara F, Olivera A, Vinatea L. (2013). Water quality and growth of Pacific white shrimp Litopenaeus vannamei (Boone) in co-culture with green seaweed Ulva lactuca (Linaeus) in intensive system. Aquaculture International 22: 497-508. 
Brown MN, Briones A, Diana J, Raskin L. (2013). Ammonia-oxidizing archaea and nitrite-oxidizing nitrospiras in the biofilter of a shrimp recirculating aquaculture system. FEMS microbiology ecology 83: 17-25.

Brunvold L, Sandaa R-A, Mikkelsen H, Welde E, Bleie H, Bergh Ø. (2007). Characterisation of bacterial communities associated with early stages of intensively reared cod (Gadus morhua) using Denaturing Gradient Gel Electrophoresis (DGGE). Aquaculture 272: 319-327.

Burford M, Thompson P, McIntosh R. (2003). Nutrient and microbial dynamics in high-intensity, zero-exchange shrimp ponds in Belize. Aquaculture 219: 393-411.

Cahill MM. (1990). Bacterial flora of fishes: A review. Microbial Ecology 19: 21-41.

Cain K, Swan C. (2010). Barrier function and immunology. Fish Physiology 30: 111-134.

Caipang CMA, Brinchmann MF, Kiron V. (2010). Antagonistic activity of bacterial isolates from intestinal microbiota of Atlantic cod, Gadus morhua, and an investigation of their immunomodulatory capabilities. Aquaculture Research 41: 249-256.

Canakci S, Kacagan M, Inan K, Belduz AO, Saha BC. (2008). Cloning, purification, and characterization of a thermostable alpha-L-arabinofuranosidase from Anoxybacillus kestanbolensis AC26Sari. Applied microbiology and biotechnology 81: 61-8.

Caporaso JG, Kuczynski J, Stombaugh J, Bittinger K, Bushman FD, Costello EK, Fierer N, Peña AG, Goodrich JK, Gordon Jl, Huttley GA, Kelley ST, Knights D, Koenig JE, Ley RE, Lozupone CA, McDonald D, Muegge BD, Pirrung M, Reeder J, Sevinsky JR, Turnbaugh PJ, Walters WA, Widmann J, Yatsunenko T, Zaneveld J, Knight R. (2010). QIIME allows analysis of high-throughput community sequencing data. Nature methods 7: 335-6.

Carda-Diéguez M, Mira A, Fouz B. (2014). Pyrosequencing survey of intestinal microbiota diversity in cultured sea bass (Dicentrarchus labrax) fed functional diets. FEMS Microbiology Ecology 87: 451-459.

Cardinali-Rezende J, Araújo JC, Almeida PGS, Chernicharo C a L, Sanz JL, Chartone-Souza E, Nascimento AM a. (2013). Organic loading rate and food-to-microorganism ratio shape prokaryotic diversity in a demoscale up-flow anaerobic sludge blanket reactor treating domestic wastewater. Antonie van Leeuwenhoek 104: 993-1003.

Carrascal OMP, Elorza MP, Restrepo GEC, Herrera CXM. (2014). Assessment of the bacterial community diversity associated with the queen conch Strombus gigas (Linnaeus, 1758) from the Caribbean coast of Colombia using denaturing gradient gel electrophoresis and culturing. Aquaculture Research 45: 773-786.

Castresana J. (2000). Selection of conserved blocks from multiple alignments for their use in phylogenetic analysis. Molecular biology and evolution 17: 540-552.

Cébron A, Berthe T, Garnier J. (2003). Nitrification and Nitrifying Bacteria in the Lower Seine River and Estuary (France) Nitrification and Nitrifying Bacteria in the Lower Seine River and Estuary ( France ). 69. e-pub ahead of print, doi: 10.1128/AEM.69.12.7091.

Chapman MG, Underwood AJ. (1999). Ecological patterns in multivariate assemblages: information and interpretation of negative values in ANOSIM tests. Marine ecology progress series 180: 257-265.

Chaucheyras-Durand F, Durand H. (2010). Probiotics in animal nutrition and health. Beneficial microbes 1: 3-9.

Chevenet F, Brun C, Bañuls A-L, Jacq B, Christen R. (2006). TreeDyn: towards dynamic graphics and annotations for analyses of trees. BMC bioinformatics 7: 439.

Clarke KR. (1993). Non-parametric multivariate analyses of changes in community structure. Australian journal of ecology.

Clements KD, Angert ER, Montgomery WL, Choat JH. (2014). Intestinal microbiota in fishes: what's known and what's not. Molecular ecology 23: 1891-8.

Corthésy B, Gaskins HR, Mercenier A. (2007). Cross-talk between probiotic bacteria and the host immune system. The Journal of nutrition 137: 781S-90S.

Crab R, Avnimelech Y, Defoirdt T, Bossier P, Verstraete W. (2007). Nitrogen removal techniques in aquaculture for a sustainable production. Aquaculture 270: 1-14.

Crab R, Defoirdt T, Bossier P, Verstraete W. (2012). Biofloc technology in aquaculture: Beneficial effects and future challenges. Aquaculture 356-357: 351-356. 
Daims H, Brühl A, Amann R, Schleifer KH, Wagner M. (1999). The domain-specific probe EUB338 is insufficient for the detection of all Bacteria: development and evaluation of a more comprehensive probe set. Systematic and applied microbiology 22: 434-444.

Delsuc F, Metcalf JL, Wegener Parfrey L, Song SJ, González A, Knight R. (2014). Convergence of gut microbiomes in myrmecophagous mammals. Molecular ecology 23: 1301-17.

DeSantis TZ, Hugenholtz P, Keller K, Brodie EL, Larsen N, Piceno YM, Phan R, Andersen GL. (2006). NAST: a multiple sequence alignment server for comparative analysis of 16S rRNA genes. Nucleic acids research 34: W3949.

Dimitroglou A, Merrifield DL, Carnevali O, Picchietti S, Avella M, Daniels C, Güroy D, Davies SJ. (2011). Microbial manipulations to improve fish health and production - A Mediterranean perspective. Fish \& shellfish immunology 30: 1-16.

Dridi B, Raoult D, Drancourt M. (2011). Archaea as emerging organisms in complex human microbiomes. Anaerobe 17: 56-63.

Dubrou S, Konjek J, Macheras E, Welté B, Guidicelli L, Chignon E, Joyeux M, Gaillard JL, Heym B, Tully T, Sapriel G. (2013). Diversity, community composition, and dynamics of nonpigmented and late-pigmenting rapidly growing mycobacteria in an urban tap water production and distribution system. Applied and environmental microbiology 79: 5498-508.

Dulger S, Demirbag Z, Belduz AO. (2004). Anoxybacillus ayderensis sp. nov. and Anoxybacillus kestanbolensis sp. nov. International journal of systematic and evolutionary microbiology 54: 1499-503.

Durand L, Zbinden M, Cueff-Gauchard V, Duperron S, Roussel EG, Shillito B, Cambon-Bonavita MA. (2010). Microbial diversity associated with the hydrothermal shrimp Rimicaris exoculata gut and occurrence of a resident microbial community. FEMS Microbiology Ecology 71: 291-303.

Ebeling JM, Timmons MB, Bisogni JJ. (2006). Engineering analysis of the stoichiometry of photoautotrophic, autotrophic, and heterotrophic removal of ammonia-nitrogen in aquaculture systems. Aquaculture 257: 346-358.

Ebrahimi S, Gabus S, Rohrbach-Brandt E, Hosseini M, Rossi P, Maillard J, Holliger C. (2010). Performance and microbial community composition dynamics of aerobic granular sludge from sequencing batch bubble column reactors operated at 20 degrees C, 30 degrees C, and 35 degrees C. Applied microbiology and biotechnology 87: 1555-68.

El-Sayed A-FM. (2006). Tilapia culture. CABI.

Ercolini D. (2004). PCR-DGGE fingerprinting: novel strategies for detection of microbes in food. Journal of microbiological methods 56: 297-314.

Ewaschuk JB, Backer JL, Churchill TA, Obermeier F, Krause DO, Madsen KL. (2007). Surface Expression of Toll-Like Receptor 9 Is Upregulated on Intestinal Epithelial Cells in Response to Pathogenic Bacterial DNA. Infection and Immunity 75: 2572-2579.

Fagundes CT, Amaral FA, Vieira AT, Soares AC, Pinho V, Nicoli JR, Vieira LQ, Teixeira MM, Souza DG. (2012). Transient TLR Activation Restores Inflammatory Response and Ability To Control Pulmonary Bacterial Infection in Germfree Mice. The Journal of Immunology 188: 1411-1420.

FAO. (2014). The State of World Fisheries and Aquaculture 2014. Rome. 223 pp.

Feng J-B, Hu C-Q, Luo P, Zhang L-P, Chen C. (2010). Microbiota of yellow grouper (Epinephelus awoora Temminck \& Schlegel, 1842) fed two different diets. Aquaculture Research 41: 1778-1790. 
Fernandez AS, Hashsham SA, Dollhopf SL, Raskin L, Glagoleva O, Dazzo FB, Hickey RF, Criddle CS, Tiedje JM. (2000). Flexible community structure correlates with stable community function in methanogenic bioreactor communities perturbed by glucose. Applied and environmental microbiology 66: 4058-67.

Fernández N, Sierra-Alvarez R, Amils R, Field J a, Sanz JL. (2009). Compared microbiology of granular sludge under autotrophic, mixotrophic and heterotrophic denitrification conditions. Water science and technology 59: 1227-36.

Fernandez-Piquer J, Bowman JP, Ross T, Tamplin ML. (2012). Molecular analysis of the bacterial communities in the live Pacific oyster (Crassostrea gigas) and the influence of postharvest temperature on its structure. Journal of applied microbiology 112: 1134-43.

Ferris M, Muyzer G, Ward D. (1996). Denaturing gradient gel electrophoresis profiles of 16S rRNA-defined populations inhabiting a hot spring microbial mat community. Applied and environmental microbiology 62: 340.

Finegold SM, Vaisanen M-L, Molitoris DR, Tomzynski TJ, Song Y, Liu C, Collins MD, Lawson PA. (2003). Cetobacterium somerae sp. nov. from human feces and emended description of the genus Cetobacterium. Systematic and applied microbiology 26: 177-81.

Finnegan L, Garcia-Melgares M, Gmerek T, Huddleston WR, Palmer A, Robertson A, Shapiro S, Unkles SE. (2011). A survey of culturable aerobic and anaerobic marine bacteria in de novo biofilm formation on natural substrates in St. Andrews Bay, Scotland. Antonie van Leeuwenhoek 100: 399-404.

Fjellheim AJ, Playfoot KJ, Skjermo J, Vadstein O. (2011). Inter-individual variation in the dominant intestinal microbiota of reared Atlantic cod (Gadus morhua L.) larvae. Aquaculture Research 1-10.

Fjellheim AJ, Playfoot KJ, Skjermo J, Vadstein O. (2007). Vibrionaceae dominates the microflora antagonistic towards Listonella anguillarum in the intestine of cultured Atlantic cod (Gadus morhua L.) larvae. Aquaculture 269: 98-106.

Flint HJ, Duncan SH, Scott KP, Louis P. (2007). Interactions and competition within the microbial community of the human colon: links between diet and health. Environmental microbiology 9: 1101-1111.

Franchini P, Fruciano C, Frickey T, Jones JC, Meyer A. (2014). The gut microbial community of Midas cichlid fish in repeatedly evolved limnetic-benthic species Pairs. PLoS ONE 9: e95027.

Friswell MK, Gika H, Stratford IJ, Theodoridis G, Telfer B, Wilson ID, McBain AJ. (2010). Site and Strain-Specific Variation in Gut Microbiota Profiles and Metabolism in Experimental Mice Ahmed N (ed). PLoS ONE 5: e8584.

Fujimura K, Okada N. (2007). Development of the embryo, larva and early juvenile of Nile tilapia Oreochromis niloticus (Pisces: Cichlidae). Developmental staging system. Development, growth \& differentiation 49: 301-24.

Garcia-Mazcorro JF, Lanerie DJ, Dowd SE, Paddock CG, Grützner N, Steiner JM, Ivanek R, Suchodolski JS, Vos W de, et al. (2011). Effect of a multi-species synbiotic formulation on fecal bacterial microbiota of healthy cats and dogs as evaluated by pyrosequencing. FEMS microbiology ecology 78: 542-54.

Gatesoupe FJ, Covès D. (2012). A spatiotemporal study of bacterial community profiles associated with Atlantic bluefin tuna larvae, Thunnus thynnus L., in three Mediterranean hatcheries. Aquaculture Research 1-13.

Gatesoupe FJJ. (1999). The use of probiotics in aquaculture. Aquaculture 180: 147-165.

Geraylou Z, Souffreau C, Rurangwa E, Maes GE, Spanier KI, Courtin CM, Delcour J a., Buyse J, Ollevier F. (2013). Prebiotic effects of arabinoxylan oligosaccharides on juvenile Siberian sturgeon (Acipenser baerii) with emphasis on the modulation of the gut microbiota using 454 pyrosequencing. FEMS Microbiology Ecology 86: 357-371.

German DP, Horn MH, Gawlicka A. Digestive enzyme activities in herbivorous and carnivorous prickleback fishes (Teleostei: Stichaeidae): ontogenetic, dietary, and phylogenetic effects. Physiological and biochemical zoology: PBZ 77: 789-804.

Gerritsen J, Smidt H, Rijkers GT, de Vos WM. (2011). Intestinal microbiota in human health and disease: the impact of probiotics. Genes \& nutrition 6: 209-40. 
Giatsis C, Ramiro-Garcia J, Abernathy J, Verreth J, Smidt H, Sipkema D, Verdegem M. (2016a). Probiotic legacy on gut microbial assembly in fish larvae. Scientific Reports (online).

Giatsis C, Sipkema D, Smidt H, Heilig H, Benvenuti G, Verreth J, Verdegem M. (2015). The impact of rearing environment on the development of gut microbiota in tilapia larvae. Scientific reports 5: 18206.

Giatsis C, Sipkema D, Smidt H, Verreth J, Verdegem M. (2014). The Colonization Dynamics of the Gut Microbiota in Tilapia Larvae. PLoS ONE 9: e103641.

Giatsis C, Verdegem M, Loo J van, Rohaan B, Verreth J, Smidt H, Ramiro-Garcia J, Sipkema D. (2016b). Steering the gut microbiome of tilapia larvae with microbial diets (under review).

Ginige MP, Keller J, Blackall LL. (2005). Investigation of an Acetate-Fed Denitrifying Microbial Community by Stable Isotope Probing , Full-Cycle rRNA Analysis, and Fluorescent In Situ Hybridization-Microutoradiography. Applied and environmental microbiology 71: 8683.

Glöckner FO, Fuchs BM, Glo FO, Amann R. (1999). Bacterioplankton Compositions of Lakes and Oceans : a First Comparison Based on Fluorescence In Situ Hybridization Bacterioplankton Compositions of Lakes and Oceans : a First Comparison Based on Fluorescence In Situ Hybridization.

Gomez de Agüero M, Ganal-Vonarburg SC, Fuhrer T, Rupp S, Uchimura Y, Li H, Steinert A, Heikenwalder M, Macpherson AJ, et al. (2016). The maternal microbiota drives early postnatal innate immune development. Science (New York, NY) 351: 1296-302.

Gómez GD, Balcázar JL. (2008). A review on the interactions between gut microbiota and innate immunity of fish. FEMS immunology and medical microbiology 52: 145-54.

Gomila M, Ramirez A, Gascó J, Lalucat J. (2008). Mycobacterium llatzerense sp. nov., a facultatively autotrophic, hydrogen-oxidizing bacterium isolated from haemodialysis water. International journal of systematic and evolutionary microbiology 58: 2769-73.

Goodhead AK, Head IM, Snape JR, Davenport RJ. (2013). Standard inocula preparations reduce the bacterial diversity and reliability of regulatory biodegradation tests. Environmental science and pollution research international In Press.

Gorley RN, Clarke RK. (2006). PRIMER v6: User manual/tutorial.

Gram L, Melchiorsen J, Bruhn JB. (2010). Antibacterial activity of marine culturable bacteria collected from a global sampling of ocean surface waters and surface swabs of marine organisms. Marine biotechnology (New York, NY) 12: 439-51.

Graue J, Engelen B, Cypionka H. (2012). Degradation of cyanobacterial biomass in anoxic tidal-flat sediments: a microcosm study of metabolic processes and community changes. The ISME journal 6: 660-9.

Green DH, Wakeley PR, Page A, Barnes A, Baccigalupi L, Ricca E, Cutting SM. (1999). Characterization of two Bacillus probiotics. Applied and environmental microbiology 65: 4288-91.

Grześkowiak $Ł$, Collado MC, Salminen S. (2012). Evaluation of aggregation abilities between commensal fish bacteria and pathogens. Aquaculture 356-357: 412-414.

Guo F, Zhang S-H, Yu X, Wei B. (2011). Variations of both bacterial community and extracellular polymers: the inducements of increase of cell hydrophobicity from biofloc to aerobic granule sludge. Bioresource technology 102: 6421-8.

Guss AM, Roeselers G, Newton ILG, Young CR, Klepac-Ceraj V, Lory S, Cavanaugh CM. (2011). Phylogenetic and metabolic diversity of bacteria associated with cystic fibrosis. The ISMEjournal 5: 20-29.

Gutierrez-Wing MT, Malone RF. (2006). Biological filters in aquaculture: Trends and research directions for freshwater and marine applications. Aquacultural Engineering 34: 163-171.

Haenen D, Zhang J, Souza da Silva C, Bosch G, van der Meer IM, van Arkel J, van den Borne JJGC, Pérez Gutiérrez O, Smidt H, Kemp B, Müller M, Hooiveld GJEJ. (2013). A diet high in resistant starch modulates microbiota composition, SCFA concentrations, and gene expression in pig intestine. The Journal of nutrition 143: 274-83.

Hahn MW, Kasalický V, Jezbera J, Brandt U, Jezberová J, Simek K. (2010). Limnohabitans curvus gen. nov., sp. nov., a planktonic bacterium isolated from a freshwater lake. International journal of systematic and evolutionary microbiology 60: 1358-65. 
Hahn MW, Scheuerl T, Jezberová J, Koll U, Jezbera J, Šimek K, Vannini C, Petroni G, Wu QL. (2012). The passive yet successful way of planktonic life: genomic and experimental analysis of the ecology of a free-living polynucleobacter population. PloS one 7: e32772.

Hahn MW, Stadler P, Wu QL, Pöckl M. (2004). The filtration-acclimatization method for isolation of an important fraction of the not readily cultivable bacteria. Journal of microbiological methods 57: 379-90.

Hamady M, Walker JJ, Harris JK, Gold NJ, Knight R. (2008). Error-correcting barcoded primers for pyrosequencing hundreds of samples in multiplex. Nature methods 5: 235-7.

Han S, Liu Y, Zhou Z, He S, Cao Y, Shi P, Yao B, Ringø E. (2010). Analysis of bacterial diversity in the intestine of grass carp (Ctenopharyngodon idellus) based on 16S rDNA gene sequences. Aquaculture Research 42: 47-56.

Hansen GH, Olafsen JA. (1999). Bacterial Interactions in Early Life Stages of Marine Cold Water Fish. Microbial Ecology 38: 1-26.

Hargreaves J a. (2006). Photosynthetic suspended-growth systems in aquaculture. Aquacultural Engineering 34: 344-363.

Hartviksen M, Vecino JLG, Ringø E, Bakke A-M, Wadsworth S, Krogdahl Å, Ruohonen K, Kettunen A. (2014). Alternative dietary protein sources for Atlantic salmon (Salmo salar L.) effect on intestinal microbiota, intestinal and liver histology and growth. Aquaculture Nutrition 20: 381-398.

He S, Zhou Z, Liu Y, Cao Y, Meng K, Shi P, Yao B, Ringø E. (2012). Do dietary betaine and the antibiotic florfenicol influence the intestinal autochthonous bacterial community in hybrid tilapia (Oreochromis niloticus $q \times$ O. aureus (J)? World Journal of Microbiology and Biotechnology 28: 785-791.

He S, Zhou Z, Liu Y, Cao Y, Meng K, Shi P, Yao B, Ringø E. (2010). Effects of the antibiotic growth promoters flavomycin and florfenicol on the autochthonous intestinal microbiota of hybrid tilapia (Oreochromis niloticus $q \times 0$. aureus ${ }^{3}$ ). Archives of Microbiology 192: 985-994.

He S, Zhou Z, Meng K, Zhao H, Yao B, Ringø E, Yoon I. (2011). Effects of dietary antibiotic growth promoter and Saccharomyces cerevisiae fermentation product on production, intestinal bacterial community, and nonspecific immunity of hybrid tilapia (Oreochromis niloticus female $\times$ Oreochromis aureus male). Journal of Animal Science 89: 84-92.

Hess-Erga O-K, Blomvågnes-Bakke B, Vadstein O. (2010). Recolonization by heterotrophic bacteria after UV irradiation or ozonation of seawater; a simulation of ballast water treatment. Water research 44: 5439-49.

Hiraishi A, Muramatsu K, Urata K. (1995). Characterization of new denitrifying Rhodobacter strains isolated from photosynthetic sludge for wastewater treatment. Journal of Fermentation and Bioengineering 79: 39-44.

Holinger EP, Ross K a, Robertson CE, Stevens MJ, Harris JK, Pace NR. (2014). Molecular analysis of point-of-use municipal drinking water microbiology. Water research 49: 225-35.

Hong HA, Khaneja R, Tam NMK, Cazzato A, Tan S, Urdaci M, Brisson A, Gasbarrini A, Barnes I, Cutting SM. (2009). Bacillus subtilis isolated from the human gastrointestinal tract. Research in microbiology 160: 134-43.

Hovanec TA, Taylor LT, Blakis A, Delong F. (1998). Nitrospira -Like Bacteria Associated with Nitrite Oxidation in Freshwater Aquaria Nitrospira -Like Bacteria Associated with Nitrite Oxidation in Freshwater Aquaria. 64.

Huber I, Spanggaard B, Appel KF, Rossen L, Nielsen T, Gram L. (2004). Phylogenetic analysis and in situ identification of the intestinal microbial community of rainbow trout (Oncorhynchus mykiss, Walbaum). Journal of Applied Microbiology 96: 117-132.

Hurlbert SHS. (1971). The nonconcept of species diversity: a critique and alternative parameters. Ecology 52: 577586.

Ingerslev HC, von Gersdorff Jørgensen L, Lenz Strube M, Larsen N, Dalsgaard I, Boye M, Madsen L. (2014). The development of the gut microbiota in rainbow trout (Oncorhynchus mykiss) is affected by first feeding and diet type. Aquaculture 424-425: 24-34.

Inui M, Murakami S, Okino S, Kawaguchi H, Vertès A a., Yukawa H. (2004). Metabolic analysis of Corynebacterium glutamicum during lactate and succinate productions under oxygen deprivation conditions. Journal of Molecular Microbiology and Biotechnology 7: 182-196. 
Itoi S, Ebihara N, Washio S, Sugita H. (2007). Nitrite-oxidizing bacteria, Nitrospira, distribution in the outer layer of the biofilm from filter materials of a recirculating water system for the goldfish Carassius auratus. Aquaculture 264: 297-308.

Jezbera J, Jezberová J, Kasalický V, Šimek K, Hahn MW. (2013). Patterns of Limnohabitans microdiversity across a large set of freshwater habitats as revealed by Reverse Line Blot Hybridization. PloS one 8: e58527.

Jones B V, Begley M, Hill C, Gahan CGM, Marchesi JR. (2008). Functional and comparative metagenomic analysis of bile salt hydrolase activity in the human gut microbiome. Proceedings of the National Academy of Sciences of the United States of America 105: 13580-5.

Joossens M, Huys G, Van Steen K, Cnockaert M, Vermeire S, Rutgeerts P, Verbeke K, Vandamme P, De Preter V. (2011). High-throughput method for comparative analysis of denaturing gradient gel electrophoresis profiles from human fecal samples reveals significant increases in two bifidobacterial species after inulintype prebiotic intake. FEMS microbiology ecology 75: 343-9.

Kahlisch L, Henne K, Gröbe L, Brettar I, Höfle MG. (2012). Assessing the viability of bacterial species in drinking water by combined cellular and molecular analyses. Microbial ecology 63: 383-97.

Kasalický V, Jezbera J, Hahn MW, Šimek K. (2013). The diversity of the Limnohabitans genus, an important group of freshwater bacterioplankton, by characterization of 35 isolated strains. PloS one 8: e58209.

Kasalický V, Jezbera J, Simek K, Hahn MW. (2010). Limnohabitans planktonicus sp. nov. and Limnohabitans parvus sp. nov., planktonic betaproteobacteria isolated from a freshwater reservoir, and emended description of the genus Limnohabitans. International journal of systematic and evolutionary microbiology 60: 2710-4.

Kawasaki H, Hoshino Y, Yamasato K. (1993). Phylogenetic diversity of phototrophic purple non-sulfur bacteria in the Proteobacteria Î group. FEMS Microbiology Letters 112: 61-66.

Keskin M, Keskin M, Rosenthal H. (1994). Pathways of bacterial contamination during egg incubation and larval rearing of turbot, Scophthalmus maximus. Journal of Applied Ichthyology 93: 1-9.

van Kessel MA, Dutilh BE, Neveling K, Kwint MP, Veltman J a, Flik G, Jetten MS, Klaren PH, Op den Camp HJ. (2011). Pyrosequencing of $16 \mathrm{~S}$ rRNA gene amplicons to study the microbiota in the gastrointestinal tract of carp (Cyprinus carpio L.). AMB Express 1:41.

Khan A, Mandal S, Samanta D, Chatterjee S, Ghosh K. (2011). Phytase-Producing Rhodococcus sp. (MTCC 9508) from Fish Gut: A Preliminary Study. Proceedings of the Zoological Society 64: 29-34.

Khan ST, Hiraishi A. (2002). Diaphorobacter nitroreducens gen nov, sp nov, a poly(3-hydroxybutyrate)-degrading denitrifying bacterium isolated from activated sludge. The Journal of general and applied microbiology 48: 299-308.

Khan ST, Horiba Y, Yamamoto M, Hiraishi A. (2002). Members of the Family Comamonadaceae as Primary Poly(3Hydroxybutyrate-co-3-Hydroxyvalerate)-Degrading Denitrifiers in Activated Sludge as Revealed by a Polyphasic Approach. Applied and environmental microbiology 68: 3206-3214.

Khardenavis A a, Kapley A, Purohit HJ. (2007). Simultaneous nitrification and denitrification by diverse Diaphorobacter sp. Applied microbiology and biotechnology 77: 403-9.

Khemkhao M, Nuntakumjorn B, Techkarnjanaruk S, Phalakornkule C. (2011). Effect of chitosan on UASB treating POME during a transition from mesophilic to thermophilic conditions. Bioresource technology 102: 467481.

Khoruts a, Sadowsky MJ. (2011). Therapeutic transplantation of the distal gut microbiota. Mucosal immunology 4: 4-7.

Kim D-H, Austin B. (2006). Innate immune responses in rainbow trout (Oncorhynchus mykiss, Walbaum) induced by probiotics. Fish \& shellfish immunology 21: 513-24.

Kim DH, Brunt J, Austin B. (2007). Microbial diversity of intestinal contents and mucus in rainbow trout (Oncorhynchus mykiss). Journal of Applied Microbiology 102: 1654-1664. 
Kim T, Yu GH, Jung J, Park H. (2010). Bacterial Community Composition and Diversity of a Full-Scale Integrated Fixed-Film Activated Sludge System as Investigated by Pyrosequencing. 20: 1717-1723.

Kim Y-J, Nguyen N-L, Weon H-Y, Yang D-C. (2013). Sediminibacterium ginsengisoli sp. nov., isolated from soil of a ginseng field, and emended descriptions of the genus Sediminibacterium and of Sediminibacterium salmoneum. International journal of systematic and evolutionary microbiology 63: 905-12.

Kohl KD, Amaya J, Passement C a., Dearing MD, McCue MD. (2014). Unique and shared responses of the gut microbiota to prolonged fasting: a comparative study across five classes of vertebrate hosts. FEMS Microbiology Ecology 90: 883-894.

Kowalchuk G, Hol W, Vanveen J. (2006). Rhizosphere fungal communities are influenced by Senecio jacobaea pyrrolizidine alkaloid content and composition. Soil Biology and Biochemistry 38: 2852-2859.

de Kreuk MK, Heijnen JJ, van Loosdrecht MCM. (2005). Simultaneous COD, nitrogen, and phosphate removal by aerobic granular sludge. Biotechnology and bioengineering 90: 761-9.

Kruse M, Keuter S, Bakker E, Spieck E, Eggers T, Lipski A. (2013). Relevance and diversity of Nitrospira populations in biofilters of brackish RAS. PloS one 8: e64737.

Kubinak JL, Round JL. (2012). Toll-like receptors promote mutually beneficial commensal-host interactions. PLoS pathogens 8: e1002785.

Kumar R, Mukherjee SC, Prasad KP, Pal AK. (2006). Evaluation of Bacillus subtilis as a probiotic to Indian major carp Labeo rohita (Ham.). Aquaculture Research 37: 1215-1221.

Kumar R, Mukherjee SC, Ranjan R, Nayak SK. (2008). Enhanced innate immune parameters in Labeo rohita (Ham.) following oral administration of Bacillus subtilis. Fish \& shellfish immunology 24: 168-72.

Laclaire LL, Facklam RR. (2000). Comparison of three commercial rapid identification systems for the unusual gram-positive cocci Dolosigranulum pigrum, Ignavigranum ruoffiae, and Facklamia species. Journal of Clinical Microbiology 38: 2037-2042.

Langlois TJ, Anderson MJ, Babcock RC, Kato S. (2006). Marine reserves demonstrate trophic interactions across habitats. Oecologia 147: 134-40.

Legendre P, Anderson MJ. (1999). Distance-based redundancy analysis: testing multispecies responses in multifactorial ecological experiments. Ecological Monographs 69: 1-24.

Legendre P, Gallagher E. (2001). Ecologically meaningful transformations for ordination of species data. Oecologia 129: $271-280$.

Lepage P, Leclerc MC, Joossens M, Mondot S, Blottière HM, Raes J, Ehrlich D, Doré J, Blottiere HM, Raes J, Ehrlich D, Dore J. (2012). A metagenomic insight into our gut's microbiome. Gut 62: 146-58.

Lever MA, Torti A, Eickenbusch P, Michaud AB, Šantl-Temkiv T, Jørgensen BB. (2015). A modular method for the extraction of DNA and RNA, and the separation of DNA pools from diverse environmental sample types. Frontiers in Microbiology 6. e-pub ahead of print, doi: 10.3389/fmicb.2015.00476.

Ley RE. (2010). Obesity and the human microbiome. Current opinion in gastroenterology 26: 5-11.

Ley RE, Bäckhed F, Turnbaugh P, Lozupone CA, Knight RD, Gordon Jl. (2005). Obesity alters gut microbial ecology. Proceedings of the National Academy of Sciences of the United States of America 102: 11070-5.

Ley RE, Hamady M, Lozupone C. (2008). Evolution of mammals and their gut microbes. Science 320: 1647-1652.

Li J, Tan B, Mai K, Ai Q. (2008). Responses and Resistance against Vibrio parahaemolyticus Induced by Probiotic Bacterium Arthrobacter XE-7 in Pacific White Shrimp, Litopenaeus vannamei. Journal of the world aquaculture society 39: 477-489.

Li J, Tan B, Mai K, Ai Q, Zhang W, Xu W, Liufu Z, Ma H. (2006). Comparative study between probiotic bacterium Arthrobacter XE-7 and chloramphenicol on protection of Penaeus chinensis post-larvae from pathogenic vibrios. Aquaculture 253: 140-147.

LiS, Sun L, Wu H, Hu Z, Liu W, LiY, Wen X. (2012a). The intestinal microbial diversity in mud crab (Scyllaparamamosain) as determined by PCR-DGGE and clone library analysis. Journal of applied microbiology 113: 1341-51. 
Li T, Long M, Gatesoupe F-J, Zhang Q, Li A, Gong X. (2014a). Comparative Analysis of the Intestinal Bacterial Communities in Different Species of Carp by Pyrosequencing. Microbial Ecology 69: 25-36.

Li W, Han L, Yu P, Ma C, Wu X, Xu J. (2014b). Nested PCR-denaturing gradient gel electrophoresis analysis of human skin microbial diversity with age. Microbiological research In Press.

Li X, Yu Y, Feng W, Yan Q, Gong Y. (2012b). Host species as a strong determinant of the intestinal microbiota of fish larvae. Journal of microbiology 50: 29-37.

Ling Z, Liu X, Chen W, Luo Y, Yuan L, Xia Y, Nelson KE, Huang S, Zhang S, Wang Y, Yuan J, Li L, Xiang C. (2013). The Restoration of the Vaginal Microbiota After Treatment for Bacterial Vaginosis with Metronidazole or Probiotics. Microbial Ecology 65: 773-780.

Liu K-F, Chiu C-H, Shiu Y-L, Cheng W, Liu C-H. (2010). Effects of the probiotic, Bacillus subtilis E20, on the survival, development, stress tolerance, and immune status of white shrimp, Litopenaeus vannamei larvae. Fish \& shellfish immunology 28: 837-44.

Liu R, Yu Z, Guo H, Liu M, Zhang H, Yang M. (2012a). Pyrosequencing analysis of eukaryotic and bacterial communities in faucet biofilms. The Science of the total environment 435-436: 124-31.

Liu R, Yu Z, Zhang H, Yang M, Shi B, Liu X. (2012b). Diversity of bacteria and mycobacteria in biofilms of two urban drinking water distribution systems. 270: 261-270.

Lozupone CA, Stombaugh J, Gonzalez A, Ackermann G, Wendel D, Vázquez-Baeza Y, Jansson JK, Gordon Jl, Knight R. (2013). Meta-analyses of studies of the human microbiota. Genome research 23: 1704-14.

Luo G, Gao Q, Wang C, Liu W, Sun D, Li L, Tan H. (2014). Growth, digestive activity, welfare, and partial costeffectiveness of genetically improved farmed tilapia (Oreochromis niloticus) cultured in a recirculating aquaculture system and an indoor biofloc system. Aquaculture 422-423: 1-7.

Ma J, Zhao B, Frear C, Zhao Q, Yu L, Li X, Chen S. (2013). Methanosarcina domination in anaerobic sequencing batch reactor at short hydraulic retention time. Bioresource technology 137: 41-50.

Van Der Maarel MJEC, Sprenger W, Haanstra R, Forney LJ. (1999). Detection of methanogenic archaea in seawater particles and the digestive tract of a marine fish species. FEMS Microbiology Letters 173: 189-194.

Machiels K, Joossens M, Sabino J, De Preter V, Arijs I, Eeckhaut V, Ballet V, Claes K, Van Immerseel F, Verbeke K, Ferrante M, Verhaegen J, Rutgeerts P, Vermeire S. (2013). A decrease of the butyrate-producing species Roseburia hominis and Faecalibacterium prausnitzii defines dysbiosis in patients with ulcerative colitis. Gut In press.

Madigan M, Cox SS, Stegeman R a. (1984). Nitrogen fixation and nitrogenase activities in members of the family Rhodospirillaceae. Journal of bacteriology 157: 73-8.

Madigan TL, Bott NJ, Torok V a, Percy NJ, Carragher JF, de Barros Lopes M a, Kiermeier A. (2014). A microbial spoilage profile of half shell Pacific oysters (Crassostrea gigas) and Sydney rock oysters (Saccostrea glomerata). Food microbiology 38: 219-27.

Di Maiuta N, Schwarzentruber P, Schenker M, Schoelkopf J. (2013). Microbial population dynamics in the faeces of wood-eating loricariid catfishes. Letters in Applied Microbiology 56: 401-407.

Mansfield GS, Desai a R, Nilson S a, Van Kessel a G, Drew MD, Hill JE. (2010). Characterization of rainbow trout (Oncorhynchus mykiss) intestinal microbiota and inflammatory marker gene expression in a recirculating aquaculture system. Aquaculture 307: 95-104.

Martínez Cruz P, Ibáñez AL, Monroy Hermosillo OA, Ramírez Saad HC. (2012). Use of probiotics in aquaculture. ISRN microbiology 2012: 916845.

Matarazzo F, Ribeiro AC, Faveri M, Taddei C, Martinez MB, Mayer MPA. (2012). The domain Archaea in human mucosal surfaces. Clinical microbiology and infection: the official publication of the European Society of Clinical Microbiology and Infectious Diseases 18: 834-40.

McDonald R, Schreier HJ, Watts JEM. (2012). Phylogenetic analysis of microbial communities in different regions of the gastrointestinal tract in Panaque nigrolineatus, a wood-eating fish. PloS one 7: e48018. 
McFall-Ngai M, Hadfield MG, Bosch TCG, Carey H V, Domazet-Lošo T, Douglas AE, Dubilier N, Eberl G, Fukami T, Gilbert SF, Hentschel U, King N, Kjelleberg S, Knoll AH, Kremer N, Mazmanian SK, Metcalf JL, Nealson K, Pierce NE, Rawls JF, Reid A, Ruby EG, Rumpho M, Sanders JG, Tautz D, Wernegreen JJ. (2013). Animals in a bacterial world, a new imperative for the life sciences. Proceedings of the National Academy of Sciences of the United States of America 110: 3229-36.

McIntosh D, Ji B, Forward BS, Puvanendran V, Boyce D, Ritchie R. (2008). Culture-independent characterization of the bacterial populations associated with cod (Gadus morhua L.) and live feed at an experimental hatchery facility using denaturing gradient gel electrophoresis. Aquaculture 275: 42-50.

Merrifield DL, Burnard D, Bradley G, Davies SJ, Baker RTM. (2009a). Microbial community diversity associated with the intestinal mucosa of farmed rainbow trout (Oncoryhnchus mykiss Walbaum). Aquaculture Research 40: 1064-1072.

Merrifield DL, Dimitroglou a, Bradley G, Baker RTM, Davies SJ. (2009b). Soybean meal alters autochthonous microbial populations, microvilli morphology and compromises intestinal enterocyte integrity of rainbow trout, Oncorhynchus mykiss (Walbaum). Journal of fish diseases 32: 755-66.

Merrifield DL, Dimitroglou A, Foey A, Davies SJ, Baker RTM, Bøgwald J, Castex M, Ringø E. (2010). The current status and future focus of probiotic and prebiotic applications for salmonids. Aquaculture 302: 1-18.

Merrifield DL, Olsen RE, Myklebust R, Ringo E. (2011). Dietary Effect of Soybean (Glycine max) Products on Gut Histology and Microbiota of Fish. In: Hany El-Shemy (ed). Soybean and Nutrition. InTech, pp 231-250.

Meziti A, Mente E, Kormas KA. (2012). Gut bacteria associated with different diets in reared Nephrops norvegicus. Systematic and applied microbiology 35: 473-82.

Montes M, Pérez MJ, Nieto TP. (1999). Numerical taxonomy of gram-negative, facultative anaerobic bacteria isolated from skin of turbot (Scophthalmus maximus) and surrounding water. Systematic and applied microbiology 22: 604-618.

Montesi A, García-Albiach R, Pozuelo MJ, Pintado C, Goñi I, Rotger R. (2005). Molecular and microbiological analysis of caecal microbiota in rats fed with diets supplemented either with prebiotics or probiotics. International Journal of Food Microbiology 98: 281-289.

Mouchet M a, Bouvier C, Bouvier T, Troussellier M, Escalas A, Mouillot D. (2012). Genetic difference but functional similarity among fish gut bacterial communities through molecular and biochemical fingerprints. FEMS Microbiology Ecology 79: 568-580.

Mudarris M, Austin B. (1988). Quantitative and qualitative studies of the bacterial microflora of turbot, Scophthalmus maximus L., gills. Journal of Fish Biology 32: 223-229.

Muegge BD, Kuczynski J, Knights D, Clemente JC, González A, Fontana L, Henrissat B, Knight R, Gordon Jl. (2011). Diet drives convergence in gut microbiome functions across mammalian phylogeny and within humans. Science 332: 970-4.

Mußmann M, Ribot M, von Schiller D, Merbt SN, Augspurger C, Karwautz C, Winkel M, Battin TJ, Martí E, Daims H. (2013). Colonization of freshwater biofilms by nitrifying bacteria from activated sludge. FEMS microbiology ecology 85: 104-15.

Muyzer G. (1999). DGGE/TGGE a method for identifying genes from natural ecosystems. Current opinion in microbiology 2: 317-22.

Muyzer G, Smalla K. (1998). Application of denaturing gradient gel electrophoresis (DGGE) and temperature gradient gel electrophoresis (TGGE) in microbial ecology. Antonie van Leeuwenhoek 73: 127-41.

Muyzer G, de Waal EC, Uitterlinden a G. (1993). Profiling of complex microbial populations by denaturing gradient gel electrophoresis analysis of polymerase chain reaction-amplified genes coding for 16S rRNA. Applied and environmental microbiology 59: 695-700.

Nakano MM, Zuber P. (1998). Anaerobic growth of a 'strict aerobe' (Bacillus subtilis). Annual review of microbiology 52: 165-90. 
Namsaraev ZB, Babasanova OB, Dunaevsky YE, Akimov VN, Barkhutova DD, Gorlenko VM, Namsaraev BB. (2010). Anoxybacillus mongoliensis sp. nov., a novel thermophilic proteinase producing bacterium isolated from alkaline hot spring, Central Mongolia. Microbiology 79: 491-499.

Navarrete P, Magne F, Araneda C, Fuentes P, Barros L, Opazo R, Espejo R, Romero J. (2012). PCR-TTGE analysis of $16 \mathrm{~S}$ rRNA from rainbow trout (Oncorhynchus mykiss) gut microbiota reveals host-specific communities of active bacteria. PLoS ONE 7: e31335-e31335.

Nayak SK. (2010a). Probiotics and immunity: a fish perspective. Fish \& shellfish immunology 29: 2-14.

Nayak SK. (2010b). Role of gastrointestinal microbiota in fish. Aquaculture Research 41: 1553-1573.

Neish AS. (2014). Mucosal immunity and the microbiome. Annals of the American Thoracic Society 11 Suppl 1: S28-32.

Newaj-Fyzul A, Adesiyun AA, Mutani A, Ramsubhag A, Brunt J, Austin B. (2007). Bacillus subtilis AB1 controls Aeromonas infection in rainbow trout (Oncorhynchus mykiss, Walbaum). Journal of applied microbiology 103: 1699-706.

Ngo Thi Hoa, Baccigalupi L, Huxham A, Smertenko A, Pham Hung Van, Ammendola S, Ricca E, Cutting SM. (2000). Characterization of Bacillus species used for oral bacteriotherapy and bacterioprophylaxis of gastrointestinal disorders. Applied and Environmental Microbiology 66: 5241-5247.

Noakes DL, Barlow G. (1976). Ontogeny of Parent-Contacting in Young Cichlasoma Citrinellum (Pisces, Cichlidae). Investigations of the Ichthyofauna of Nicaraguan Lakes.

Nubel U, Engelen B, Felske A, Snaidr J, Wieshuber A, Amann Rl, Ludwig W, Backhaus H. (1996). Sequence heterogeneities of genes encoding $16 \mathrm{~S}$ rRNAs in Paenibacillus polymyxa detected by temperature gradient gel electrophoresis. J Bacteriol 178: 5636-5643.

O'Hara AM, Shanahan F. (2006). The gut flora as a forgotten organ. EMBO reports 7: 688-93.

Olafsen JA. (2001). Interactions between fish larvae and bacteria in marine aquaculture. Aquaculture 200: $223-247$.

Oliveros JC. (2007). VENNY. An interactive tool for comparing lists with Venn Diagrams. BioinfoGP of CNB-CSIC. http://bioinfogp.cnnb.csic.es/tools/venny/index.html.

Op den Camp HJM, Jetten MSM, Strous M. (2007). Anammox. In: Biology of the Nitrogen Cycle. Elsevier, pp $245-262$.

Özen Al, Ussery DW. (2012). Defining the Pseudomonas genus: where do we draw the line with Azotobacter? Microbial ecology 63: 239-48.

Paungfoo C, Prasertsan P, Burrell PC, Intrasungkha N, Blackall LL. (2007). Nitrifying Bacterial Communities in an Aquaculture Wastewater Treatment System Using Fluorescence In Situ Hybridization ( FISH ), 16S rRNA Gene Cloning, and Phylogenetic Analysis. 97: 985-990.

Pérez Alonso a. JJ, Husein-El Ahmed H, Del Olmo Rivas C, Caballero Marcos L, Pérez Ramon J a. A. (2012). Facklamia sourekii necrotizing gangrene. Médecine et Maladies Infectieuses 42: 283-284.

PérezT, Balcázar JL, Ruiz-Zarzuela I, Halaihel N, Vendrell D, de Blas I, Múzquiz JL. (2010). Host-microbiota interactions within the fish intestinal ecosystem. Mucosal immunology 3: 355-360.

Pérez-Sánchez T, Ruiz-Zarzuela I, de Blas I, Balcázar JL. (2014). Probiotics in aquaculture: a current assessment. Reviews in Aquaculture 6: 133-146.

Pérez-Zánchez T, Ruiz-zarzuela I, Blas I De, Balcazar JL, Pérez-Sánchez T, Ruiz-zarzuela I, de Blas I, Balcázar JL, PérezZánchez T, Ruiz-zarzuela I, Blas I De, Balcazar JL. (2013). Probiotics in aquaculture: a current assessment. Reviews in Aquaculture 5: 1-14.

Piterina A V, Bartlett J, Pembroke JT. (2012). Phylogenetic analysis of the bacterial community in a full scale autothermal thermophilic aerobic digester (ATAD) treating mixed domestic wastewater sludge for land spread. Water research 46: 2488-504. 
Pond MJ, Stone DM, Alderman DJ. (2006). Comparison of conventional and molecular techniques to investigate the intestinal microflora of rainbow trout (Oncorhynchus mykiss). Aquaculture 261: 194-203.

Pruesse E, Peplies J, Glöckner FO. (2012). SINA: accurate high-throughput multiple sequence alignment of ribosomal RNA genes. Bioinformatics (Oxford, England) 28: 1823-1829.

Qu J, Yuan H. (2008). Sediminibacterium salmoneum gen. nov., sp. nov., a member of the phylum Bacteroidetes isolated from sediment of a eutrophic reservoir. International journal of systematic and ... 2191-2194.

Quan Z-X, Im W-T, Lee S-T. (2006). Azonexus caeni sp. nov., a denitrifying bacterium isolated from sludge of a wastewater treatment plant. International journal of systematic and evolutionary microbiology 56: 1043-6.

Quast C, Pruesse E, Yilmaz P, Gerken J, Schweer T, Yarza P, Peplies J, Glöckner FO. (2013). The SILVA ribosomal RNA gene database project: improved data processing and web-based tools. Nucleic acids research 41: D5906.

Raida MK, Larsen JL, Nielsen ME, Buchmann K. (2003). Enhanced resistance of rainbow trout, Oncorhynchus mykiss (Walbaum), against Yersinia ruckeri challenge following oral administration of Bacillus subtilis and $B$. licheniformis (BioPlus2B). Journal of Fish Diseases 26: 495-498.

Ramiro-Garcia J, Hermes GDA, Giatsis C, Sipkema D, Zoetendal EG, Schaap PJ, Smidt H. (2016). NG-Tax, a highly accurate and validated pipeline for analysis of $16 \mathrm{~S}$ rRNA amplicons from complex biomes. F1000 Research 5: 1791.

Ramos M a., Weber B, Gonçalves JF, Santos G a., Rema P, Ozório RO a. (2013). Dietary probiotic supplementation modulated gut microbiota and improved growth of juvenile rainbow trout (Oncorhynchus mykiss). Comparative Biochemistry and Physiology - A Molecular and Integrative Physiology 166: 302-307.

Rasolofo EA, St-Gelais D, LaPointe G, Roy D. (2010). Molecular analysis of bacterial population structure and dynamics during cold storage of untreated and treated milk. International Journal of Food Microbiology 138: $108-118$.

Rawls JF. (2007). Enteric infection and inflammation alter gut microbial ecology. Cell host \& microbe 2: 73-74.

Rawls JF, Mahowald MA, Ley RE, Gordon J. (2006). Reciprocal Gut Microbiota Transplants from Zebrafish and Mice to Germ-free Recipients Reveal Host Habitat Selection. Cell 127: 423-433.

Rawls JF, Samuel BS, Gordon JI. (2004). Gnotobiotic zebrafish reveal evolutionarily conserved responses to the gut microbiota. Proceedings of the National Academy of Sciences of the United States of America 101: 4596-601.

Ray a. K, Ghosh K, Ringø E. (2012). Enzyme-producing bacteria isolated from fish gut: a review. Aquaculture Nutrition 18: 465-492.

Ray AJ, Dillon KS, Lotz JM. (2011). Water quality dynamics and shrimp (Litopenaeus vannamei) production in intensive, mesohaline culture systems with two levels of biofloc management. Aquacultural Engineering 45: $127-136$.

Reid HI, Treasurer JW, Adam B, Birkbeck TH. (2009). Analysis of bacterial populations in the gut of developing cod larvae and identification of Vibrio logei, Vibrio anguillarum and Vibrio splendidus as pathogens of cod larvae. Aquaculture 288: 36-43.

Reitan KI, Natvik CM, Vadstein O. (1998). Drinking rate, uptake of bacteria and microalgae in turbot larvae. Journal of Fish Biology 53: 1145-1154.

Riddle MR, Baxter BK, Avery BJ. (2013). Molecular identification of microorganisms associated with the brine shrimp Artemia franciscana. Aquatic biosystems 9: 7.

Ringo E, Birkbeck TH. (1999). Intestinal microflora of fish larvae and fry. Aquaculture research 30: $73-93$.

Ringø E, Sperstad S, Kraugerud OF, Krogdahl Å. (2008). Use of 16 S rRNA gene sequencing analysis to characterize culturable intestinal bacteria in Atlantic salmon (Salmo salar) fed diets with cellulose or non-starch polysaccharides from soy. Aquaculture Research 39: 1087-1100. 
Ringo E, Sperstad S, Myklebust R, Mayhew TM, Olsen RE. (2006). The effect of dietary inulin on aerobic bacteria associated with hindgut of Arctic charr (Salvelinus alpinus L.). Aquaculture Research 37: 891-897.

Ringø E, Sperstad S, Myklebust R, Refstie S, Krogdahl Å. (2006). Characterisation of the microbiota associated with intestine of Atlantic cod (Gadus morhua L.). Aquaculture 261: 829-841.

Ringø E, Strøm E, Tabachek J. (1995). Intestinal microflora of salmonids: a review. Aquaculture Research 26: 773-789.

Road SP. (1987). Anaerobic respiration in the Rhodospirillaceae " characterisation of pathways and evaluation of roles in redox balancing during photosynthesis. 46: 117-143.

Robertson PAW, O'Dowd C, Burrells C, Williams P, Austin B. (2000). Use of Carnobacterium sp. as a probiotic for Atlantic salmon (Salmo salar L.) and rainbow trout (Oncorhynchus mykiss, Walbaum). Aquaculture 185: 235-243.

Roeselers G, Mittge EK, Stephens WZ, Parichy DM, Cavanaugh CM, Guillemin K, Rawls JF. (2011). Evidence for a core gut microbiota in the zebrafish. The ISMEjournal 5: 1595-608.

Romero J, Navarrete P. (2006). 16S rDNA-based analysis of dominant bacterial populations associated with early life stages of coho salmon (Oncorhynchus kisutch). Microbial ecology 51: 422-30.

Ronquist F, Teslenko M, van der Mark P, Ayres DL, Darling A, Höhna S, Larget B, Liu L, Suchard M a, Huelsenbeck JP. (2012). MrBayes 3.2: efficient Bayesian phylogenetic inference and model choice across a large model space. Systematic biology 61: 539-542.

Roth E, Schwenninger SM, Eugster-Meier E, Lacroix C. (2011). Facultative anaerobic halophilic and alkaliphilic bacteria isolated from a natural smear ecosystem inhibit Listeria growth in early ripening stages. International Journal of Food Microbiology 147: 26-32.

Rurangwa E, Sipkema D, Kals J, Ter Veld M, Forlenza M, Bacanu GM, Smidt H, Palstra AP. (2015). Impact of a novel protein meal on the gastrointestinal microbiota and the host transcriptome of larval zebrafish Danio rerio. Frontiers in physiology 6: 133.

Rurangwa E, Verdegem MCJJ. (2014). Microorganisms in recirculating aquaculture systems and their management. Reviews in Aquaculture 5: 1-14.

Sadaie T, Sadaie A, Takada M, Hamano K, Ohnishi J, Ohta N, Matsumoto K, Sadaie Y. (2007). Reducing Sludge Production and the Domination of Comamonadaceae by Reducing the Oxygen Supply in the Wastewater Treatment Procedure of a Food-Processing Factory. Bioscience, Biotechnology, and Biochemistry 71: 791799.

Saito R, Smoot ME, Ono K, Ruscheinski J, Wang P, Lotia S, Pico AR, Bader GD, Ideker T. (2012). A travel guide to Cytoscape plugins. Nature methods 9: 1069-1076.

Salinas I, Cuesta A, Esteban MA, Meseguer J. (2005). Dietary administration of Lactobacillus delbrüeckii and Bacillus subtilis, single or combined, on gilthead seabream cellular innate immune responses. Fish \& shellfish immunology 19: 67-77.

Samuel BS, Hansen EE, Manchester JK, Coutinho PM, Henrissat B, Fulton R, Latreille P, Kim K, Wilson RK, Gordon J. (2007). Genomic and metabolic adaptations of Methanobrevibacter smithii to the human gut. Proceedings of the National Academy of Sciences of the United States of America 104: 10643-8.

Sanchez LM, Wong WR, Riener RM, Schulze CJ, Linington RG. (2012). Examining the fish microbiome: vertebratederived bacteria as an environmental niche for the discovery of unique marine natural products. PloS one 7: e35398.

Sanguinetti CJ, Neto ED, Simpson a. JG. (1994). Rapid silver staining and recovery of PCR products separated on polyacrylamide gels. BioTechniques 17: 914-21.

Satoh H, Nakamura Y, Ono H, Okabe S. (2003). Effect of oxygen concentration on nitrification and denitrification in single activated sludge flocs. Biotechnology and bioengineering 83: 604-7.

Schreier HJ, Mirzoyan N, Saito K. (2010). Microbial diversity of biological filters in recirculating aquaculture systems. Current opinion in biotechnology 21: 318-25.

De Schryver P, Crab R, Defoirdt T, Boon N, Verstraete W. (2008). The basics of bio-flocs technology: The added value for aquaculture. Aquaculture 277: 125-137. 
De Schryver P, Vadstein O. (2014). Ecological theory as a foundation to control pathogenic invasion in aquaculture. The ISME journal 8: 2360-8.

Scott KP, Duncan SH, Louis P, Flint HJ. (2011). Nutritional influences on the gut microbiota and the consequences for gastrointestinal health. Biochemical Society transactions 39: 1073-8.

Sekirov I, Russell SL, Antunes LCM, Finlay BB. (2010). Gut microbiota in health and disease. Physiological reviews 90: 859-904.

Serkebaeva YM, Kim Y, Liesack W, Dedysh SN. (2013). Pyrosequencing-based assessment of the bacteria diversity in surface and subsurface peat layers of a northern wetland, with focus on poorly studied phyla and candidate divisions. Neufeld J (ed). PloS one 8: e63994.

Sethi S, Datta A, Gupta BL, Gupta S. (2013). Optimization of Cellulase Production from Bacteria Isolated from Soil. ISRN Biotechnology 2013: 1-7.

Sharifuzzaman SM, Al-Harbi AH, Austin B. (2014). Characteristics of growth, digestive system functionality, and stress factors of rainbow trout fed probiotics Kocuria SM1 and Rhodococcus SM2. Aquaculture 418-419: 55-61.

Sheu S-Y, Chen Y-L, Young C-C, Chen W-M. (2013). Lacibacterium aquatile gen. nov., sp. nov., a new member of the family Rhodospirillaceae isolated from a freshwater lake. International journal of systematic and evolutionary microbiology 63: 4797-804.

da Silva KR, Wasielesky W, Abreu PC. (2013). Nitrogen and Phosphorus Dynamics in the Biofloc Production of the Pacific White Shrimp, Litopenaeus vannamei. Journal of the World Aquaculture Society 44: 30-41.

Šimek K, Kasalický V, Jezbera J, Horňák K, Nedoma J, Hahn MW, Bass D, Jost S, Boenigk J. (2013). Differential freshwater flagellate community response to bacterial food quality with a focus on Limnohabitans bacteria. The ISME journal 7: 1519-30.

Singleton DR, Richardson SD, Aitken MD. (2011). Pyrosequence analysis of bacterial communities in aerobic bioreactors treating polycyclic aromatic hydrocarbon-contaminated soil. Biodegradation 22: 1061-73.

Situmorang ML, Dierckens K, Mlingi FT, Van Delsen B, Bossier P. (2014). Development of a bacterial challenge test for gnotobiotic Nile tilapia Oreochromis niloticus larvae. Diseases of Aquatic Organisms 109: 23-34.

Skjermo J, Bakke I, Dahle SW, Vadstein O. (2015). Probiotic strains introduced through live feed and rearing water have low colonizing success in developing Atlantic cod larvae. Aquaculture 438: 17-23.

Smith DJ, Timonen HJ, Jaffe DA, Griffin DW, Birmele MN, Perry KD, Ward PD, Roberts MS. (2013). Intercontinental dispersal of bacteria and archaea by transpacific winds. Applied and environmental microbiology 79: 1134-9.

Smriga S, Sandin S a, Azam F. (2010). Abundance, diversity, and activity of microbial assemblages associated with coral reef fish guts and feces. FEMS microbiology ecology 73: 31-42.

Sommer F, Bäckhed F. (2013). The gut microbiota--masters of host development and physiology. Nature reviews Microbiology 11: 227-38.

Song SK, Beck BR, Kim D, Park J, Kim J, Kim HD, Ringø E. (2014). Prebiotics as immunostimulants in aquaculture: a review. Fish \& shellish immunology 40: 40-8.

Spanggaard B, Huber I, Nielsen J, Nielsen T, Appel K., Gram L. (2000). The microflora of rainbow trout intestine: a comparison of traditional and molecular identification. Aquaculture 182: 1-15.

Standen BT, Rodiles A, Peggs DL, Davies SJ, Santos GA, Merrifield DL. (2015). Modulation of the intestinal microbiota and morphology of tilapia, Oreochromis niloticus, following the application of a multi-species probiotic. Applied microbiology and biotechnology 99: 8403-17.

Stapleton RD, Taylor DE, Sayler GS. (2000). Quantification of Hyphomicrobium Populations in Activated Sludge from an Industrial Wastewater Treatment System as Determined by 16S rRNA Analysis Quantification of Hyphomicrobium Populations in Activated Sludge from an Industrial Wastewater Treatment Sys. e-pub ahead of print, doi: 10.1128/AEM.66.3.1167-1174.2000.Updated.

Steinum T, Sjåstad K, Falk K, Kvellestad A, Colquhoun DJJ. (2009). An RT PCR-DGGE survey of gill-associated bacteria in Norwegian seawater-reared Atlantic salmon suffering proliferative gill inflammation. Aquaculture 293: 172-179.

Sugita H, Mizuki H, Itoi S. (2012). Diversity of siderophore-producing bacteria isolated from the intestinal tracts of fish along the Japanese coast. Aquaculture Research 43: 481-488. 
Sugita H, Nakamura H, Shimada T. (2005). Microbial communities associated with filter materials in recirculating aquaculture systems of freshwater fish. Aquaculture 243: 403-409.

Sugita H, Shibuya K, Shimooka H, Deguchi Y. (1996). Antibacterial abilities of intestinal bacteria in freshwater cultured fish. Aquaculture 145: 195-203.

Sullam KE, Essinger SD, Lozupone CA, O'Connor MP, Rosen GL, Knight R, Kilham SS, Russel JA. (2012). Environmental and ecological factors that shape the gut bacterial communities of fish: a meta-analysis. Molecular Ecology 21: 3363-3378.

Svanevik CS, Lunestad BT. (2011). Characterisation of the microbiota of Atlantic mackerel (Scomber scombrus). International journal of food microbiology 151: 164-70.

Tabrez Khan S, Hiraishi a. (2001). Isolation and characterization of a new poly(3-hydroxybutyrate)-degrading, denitrifying bacterium from activated sludge. FEMS microbiology letters 205: 253-7.

Takahashi M, Yamada T, Tanno M, Tsuji H, Hiraishi A. (2011). Nitrate Removal Efficiency and Bacterial Community Dynamics in Denitrification Processes Using Poly (L-lactic acid) as the Solid Substrate. Microbes and Environments 26: 212-219.

Takamatsu D, Ide H, Osaki M, Sekizaki T. (2006). Identification of Facklamia sourekii from a lactating cow. The Journal of veterinary medical science / the Japanese Society of Veterinary Science 68: 1225-1227.

Tamaki H, Sekiguchi Y, Hanada S, Nomura N, Matsumura M, Nakamura K, Kamagata Y. (2005). Comparative Analysis of Bacterial Diversity in Freshwater Sediment of a Shallow Eutrophic Lake by Molecular and Improved Cultivation-Based Techniques Comparative Analysis of Bacterial Diversity in Freshwater Sediment of a Shallow Eutrophic Lake by Molecul. e-pub ahead of print, doi: 10.1128/AEM.71.4.2162.

Tang Y, Tao P, Tan J, Mu H, Peng L, Yang D, Tong S, Chen L. (2014). Identification of bacterial community composition in freshwater aquaculture system farming of Litopenaeus vannamei reveals distinct temperature-driven patterns. International journal of molecular sciences 15: 13663-80.

Tannock GW, Fuller R, Smith SL, Hall MA. (1990). Plasmid profiling of members of the family Enterobacteriaceae, lactobacilli, and bifidobacteria to study the transmission of bacteria from mother to infant. Journal of clinical microbiology 28: 1225-8.

Temmerman R, Scheirlinck I. (2003). Culture-independent analysis of probiotic products by denaturing gradient gel electrophoresis. Applied and environmental microbiology 69: 220.

Third Ka, Burnett N, Cord-Ruwisch R. (2003). Simultaneous nitrification and denitrification using stored substrate (PHB) as the electron donor in an SBR. Biotechnology and bioengineering 83: 706-20.

Thompson A, Van Moorlehem E, Aich P. (2010). Probiotic-Induced Priming of Innate Immunity to Protect Against Rotaviral Infection. Probiotics and Antimicrobial Proteins 2: 90-97.

Thompson CL. (2014). Analysis of Community Dynamics in Environmental Samples Using Denaturing Gradient Gel Electrophoresis. Humana Press: Totowa, NJ.

Tinh NTN, Asanka Gunasekara R a YS, Boon N, Dierckens K, Sorgeloos P, Bossier P. (2007). N-acyl homoserine lactone-degrading microbial enrichment cultures isolated from Penaeus vannamei shrimp gut and their probiotic properties in Brachionus plicatilis cultures. FEMS microbiology ecology 62: 45-53.

Tkavc R, Ausec L, Oren A, Gunde-Cimerman N. (2011). Bacteria associated with Artemia spp. along the salinity gradient of the solar salterns at Eilat (Israel). FEMS Microbiology Ecology 77: 310-321.

Toivonen RK, Emani R, Munukka E, Rintala A, Laiho A, Pietilä S, Pursiheimo JP, Soidinsalo P, Linhala M, Eerola E, Huovinen P, Hänninen A. (2014). Fermentable fibres condition colon microbiota and promote diabetogenesis in NOD mice. Diabetologia 57: 2183-92.

Torrentó C, Urmeneta J, Otero N, Soler A, Viñas M, Cama J. (2011). Enhanced denitrification in groundwater and sediments from a nitrate-contaminated aquifer after addition of pyrite. Chemical Geology 287: 90-101.

Tourlomousis P, Kemsley EK, Ridgway KP, Toscano MJ, Humphrey TJ, Narbad A. (2010). PCR-denaturing gradient gel electrophoresis of complex microbial communities: a two-step approach to address the effect of gel-togel variation and allow valid comparisons across a large dataset. Microbial ecology 59: 776-86. 
Tsai Y-P, You S-J, Pai T-Y, Chen K-W. (2005). Effect of cadmium on composition and diversity of bacterial communities in activated sludges. International Biodeterioration \& Biodegradation 55: 285-291.

Tseng D-Y, Ho P-L, Huang S-Y, Cheng S-C, Shiu Y-L, Chiu C-S, Liu C-H. (2009). Enhancement of immunity and disease resistance in the white shrimp, Litopenaeus vannamei, by the probiotic, Bacillus subtilis E20. Fish \& shellfish immunology 26: 339-44.

Turnbaugh PJ, Gordon J. (2009). The core gut microbiome, energy balance and obesity. The Journal of physiology 587: 4153-8.

Turnbaugh PJ, Hamady M, Yatsunenko T, Cantarel BL, Duncan A, Ley RE, Sogin ML, Jones WJ, Roe B a, Affourtit JP, Egholm M, Henrissat B, Heath AC, Knight R, Gordon Jl. (2009a). A core gut microbiome in obese and lean twins. Nature 457: 480-4.

Turnbaugh PJ, Ridaura VK, Faith JJ, Rey FE, Knight R, Gordon Jl. (2009b). The effect of diet on the human gut microbiome: a metagenomic analysis in humanized gnotobiotic mice. Science translational medicine 1: 6ra14.

Tytler P, Tatner M, Findlay C. (1990). The ontogeny of drinking in the rainbow trout, Oncorhynchus mykiss (Walbaum). Journal of Fish Biology 36: 867-875.

Ultee a., Souvatzi N, Maniadi K, Konig H. (2004). Identification of the culturable and nonculturable bacterial population in ground water of a municipal water supply in Germany. Journal of Applied Microbiology $\mathbf{9 6}$ : 560-568.

US Environmental Protection Agency. (2002). US EPA: Methods for Measuring the Acute Toxicity of Effluents and Receiving Waters to Freshwater and Marine Organisms. Environmental Protection 232: 266.

Vadstein O, Bergh $\varnothing$, Gatesoupe F-J, Galindo-Villegas J, Mulero V, Picchietti S, Scapigliati G, Makridis P, Olsen Y, Dierckens K, Defoirdt T, Boon N, De Schryver P, Bossier P. (2013). Microbiology and immunology of fish larvae. Reviews in Aquaculture 5: S1-S25.

Vanhoutte T, Huys G, Brandt E, Swings J. (2004). Temporal stability analysis of the microbiota in human feces by denaturing gradient gel electrophoresis using universal and group-specific $16 \mathrm{~S}$ rRNA gene primers. FEMS microbiology ecology 48: 437-46.

Vannini C, Pöckl M, Petroni G, Wu QL, Lang E, Stackebrandt E, Schrallhammer M, Richardson PM, Hahn MW. (2007). Endosymbiosis in statu nascendi: close phylogenetic relationship between obligately endosymbiotic and obligately free-living Polynucleobacter strains (Betaproteobacteria). Environmental microbiology 9: 347-59.

Vaseeharan B, Ramasamy P. (2003). Control of pathogenic Vibrio spp. by Bacillus subtilis BT23, a possible probiotic treatment for black tiger shrimp Penaeus monodon. Letters in Applied Microbiology 36: 83-87.

Verschuere L, Dhont J, Sorgeloos P, Verstraete W. (1997). Monitoring Biolog patterns and r/K-strategists in the intensive culture of Artemia juveniles. Journal of Applied Microbiology 83: 603-612.

Verschuere L, Rombaut G, Sorgeloos P, Verstraete W. (2000). Probiotic Bacteria as Biological Control Agents in Aquaculture. Microbiology and Molecular Biology Reviews 64: 655-671.

Vílchez R, Gómez-Silván C, Purswani J, González-López J, Rodelas B. (2011). Characterization of bacterial communities exposed to $\mathrm{Cr}(\mathrm{III})$ and $\mathrm{Pb}(\mathrm{II})$ in submerged fixed-bed biofilms for groundwater treatment. Ecotoxicology (London, England) 20: 779-92.

Walker AW, Ince J, Duncan SH, Webster LM, Holtrop G, Ze X, Brown D, Stares MD, Scott P, Bergerat A, Louis P, McIntosh F, Johnstone AM, Lobley GE, Parkhill J, Flint HJ. (2011). Dominant and diet-responsive groups of bacteria within the human colonic microbiota. The ISME journal 5: 220-30. 
Wang B, Xiao BB, Shang CG, Wang K, Na RS, Nu XX, Liao Q. (2014). Molecular analysis of the relationship between specific vaginal bacteria and bacterial vaginosis metronidazole therapy failure. European Journal of Clinical Microbiology \& Infectious Diseases 33: 1749-56.

Wang C, Zhu G, Wang Y, Wang S, Yin C. (2013). Nitrous oxide reductase gene (nosZ) and N2O reduction along the littoral gradient of a eutrophic freshwater lake. Journal of Environmental Sciences (China) 25: 44-52.

Wang H, Hu C, Hu X, Yang M, Qu J. (2012). Effects of disinfectant and biofilm on the corrosion of cast iron pipes in a reclaimed water distribution system. Water research 46: 1070-8.

Wang Q, Garrity GM, Tiedje JM, Cole JR. (2007). Naive Bayesian classifier for rapid assignment of rRNA sequences into the new bacterial taxonomy. Applied and Environmental Microbiology 73: 5261-5267.

Wang Y, Hammes F, Boon N, Chami M, Egli T. (2009). Isolation and characterization of low nucleic acid (LNA)content bacteria. The ISME journal 3: 889-902.

Wang Y-B, Li J-R, Lin J. (2008). Probiotics in aquaculture: Challenges and outlook. Aquaculture 281: 1-4.

Weissbrodt DG, Schneiter GS, Fürbringer J-M, Holliger C. (2013). Identification of trigger factors selecting for polyphosphate- and glycogen-accumulating organisms in aerobic granular sludge sequencing batch reactors. Water research 47: 7006-18.

Wendisch VF, De Graaf A a., Sahm H, Eikmanns BJ. (2000). Quantitative determination of metabolic fluxes during coutilization of two carbon sources: Comparative analyses with Corynebacterium glutamicum during growth on acetate and/or glucose. Journal of Bacteriology 182: 3088-3096.

Wilson J, Castro L. (2010). Morphological diversity of the gastrointestinal tract in fishes. Fish Physiology 30: 1-55.

Wong S, Rawls JF. (2012). Intestinal microbiota composition in fishes is influenced by host ecology and environment. Molecular Ecology 21: 3100-3102.

Wong S, Waldrop T, Summerfelt S, Davidson J, Barrows F, Kenney BB, Welch T, Wiens GD, Snekvi K, Rawls JF, Good C. (2013). Aquacultured Rainbow Trout (Oncorhynchus mykiss) Possess a Large Core Intestinal Microbiota That Is Resistant to Variation in Diet and Rearing Density. Applied and Environmental Microbiology 79: 4974-4984.

Wu QL, Hahn MW. (2006). High predictability of the seasonal dynamics of a species-like Polynucleobacter population in a freshwater lake. Environmental microbiology 8: 1660-6.

Wu S, Gao T, Zheng Y, Wang W, Cheng Y, Wang G. (2010). Microbial diversity of intestinal contents and mucus in yellow catfish (Pelteobagrus fulvidraco). Aquaculture 303: 1-7.

Wu S, Tian J, Wang G, Li W, Zou H. (2012a). Characterization of bacterial community in the stomach of yellow catfish (Pelteobagrus fulvidraco). World journal of microbiology \& biotechnology 28: 2165-74.

Wu S, Wang G, Angert ER, Wang W, Li W, Zou H. (2012b). Composition, diversity, and origin of the bacterial community in grass carp intestine. Bereswill S (ed). PloS one 7: e30440.

Wu S-G, Tian J-Y, Gatesoupe F-J, Li W-X, Zou H, Yang B-J, Wang G-T. (2013). Intestinal microbiota of gibel carp (Carassius auratus gibelio) and its origin as revealed by 454 pyrosequencing. World journal of microbiology \& biotechnology 29: 1585-95.

Xia Z, Zhu M, Zhang Y. (2014). Effects of the probiotic Arthrobacter sp. CW9 on the survival and immune status of white shrimp (Penaeus vannamei). Letters in applied microbiology 58: 60-4.

Yan Q, van der Gast CJ, Yu Y. (2012). Bacterial community assembly and turnover within the intestines of developing zebrafish. PLoS ONE 7: e30603.

Yang Y, Wang X, Shi J, Li J. (2012). The influence of the discharging sewage on microbial diversity in sludge from Dongting Lake. World journal of microbiology \& biotechnology 28: 421-30. 
Yassin a. F, Kroppenstedt RM, Ludwig W. (2003). Corynebacterium glaucum sp. nov. International Journal of Systematic and Evolutionary Microbiology 53: 705-709.

Ye L, Zhang T. (2013). Bacterial communities in different sections of a municipal wastewater treatment plant revealed by $16 \mathrm{~S}$ rDNA 454 pyrosequencing. Applied microbiology and biotechnology 97: 2681-90.

Yin Y, Lei F, Zhu L, Li S, Wu Z, Zhang R, Gao GF, Zhu B, Wang X. (2010). Exposure of different bacterial inocula to newborn chicken affects gut microbiota development and ileum gene expression. The ISME journal 4: 367-376.

Zapata A, Diez B, Cejalvo T, Gutiérrez-de Frías C, Cortés A. (2006). Ontogeny of the immune system of fish. Fish \& shellfish immunology 20: 126-36.

ZengY, KasalickýV, ŠimekK, Koblížeka M. (2012). Genome sequences of two freshwater betaproteobacterial isolates, Limnohabitans species strains Rim 28 and Rim47, indicate their capabilities as both photoautotrophs and ammonia oxidizers. Journal of bacteriology 194: 6302-3.

Zhang L, Mai K, Tan B, Ai Q, Qi C, Xu W, Zhang W, Liufu Z, Wang X, Ma H. (2009). Effects of dietary administration of probiotic Halomonas sp. B12 on the intestinal microflora, immunological parameters, and midgut histological structure of shrimp, Fenneropenaeus chinensis. Journal of the World Aquaculture Society 40: 58-66.

Zhang M, Sun Y, Chen K, Yu N, Zhou Z, Chen L, Du Z, Li E. (2014). Characterization of the intestinal microbiota in Pacific white shrimp, Litopenaeus vannamei, fed diets with different lipid sources. Aquaculture 434: 449-455.

Zhang T, Shao M-F, Ye L. (2012). 454 Pyrosequencing Reveals Bacterial Diversity of Activated Sludge From 14 Sewage Treatment Plants. The ISME journal 6: 1137-47.

Zhao J-S, Manno D, Hawari J. (2009). Psychrilyobacter atlanticus gen. nov., sp. nov., a marine member of the phylum Fusobacteria that produces $\mathrm{H} 2$ and degrades nitramine explosives under low temperature conditions. International journal of systematic and evolutionary microbiology 59: 491-7.

Zhao L, Wang G, Siegel P, He C, Wang H, Zhao W, Zhai Z, Tian F, Zhao J, Zhang H, Sun Z, Chen W, Zhang Y, Meng H. (2013). Quantitative genetic background of the host influences gut microbiomes in chickens. Scientific reports 3: 1163.

Zhou ZG, He S, Liu Y, Shi P, Huang G, Yao B. (2009). The effects of dietary yeast culture or short-chain fructooligosaccharides on the intestinal autochthonous bacterial communities in juvenile hybrid tilapia, oreochromis niloticus x oreochromis aureus. Journal of the World Aquaculture Society 40: 450-459. 



\section{Summary}

Aquaculture has realized considerable growth over the past years while the world demand on seafood has been increasing. As aquaculture intensifies, the production sector needs to tackle major bottlenecks related to suboptimal growth and high and unpredictable mortality, especially in larval cultures. Attempts in overcoming those problems mostly focused on broodstock management, optimization of water quality, nutrition and manufacturing technology of the feeds. However, microbial processes might also be involved.

Fish-microbe interactions are closely related to overall fish health. Innate immune response and nutrient metabolism depend on microorganisms that colonize the gastrointestinal tract. Therefore it is important to understand the underlying mechanisms of microbial colonization in the fish gut, which will lead to a healthy and resilient microbial community (MC) and thus contribute to fish health.

The goal of this thesis was to investigate the role of water and feed microbial communities on shaping gut communities during early development of fish.

In chapter $\mathbf{2}$ we characterized the variation in MC composition between identically reared individuals to determine the contribution of stochasticity to the overall variation. For this, we quantified the spatio-temporal variation of gut microbiota of Nile tilapia larvae, reared in recirculating or active suspension systems (RAS vs. AS). Individuals were reared for six weeks within the same or in replicate tanks, and within replicate and different production systems i.e. RAS and AS. Highly similar MCs developed in the gut when larvae shared the same water and diet. Rearing larvae in replicate production systems resulted in significantly different gut communities indicating that compositional replication of the MCs of an ecosystem is not fully predictable.

In chapter 3 the relative contribution of water and feed microbiota to the development of gut MCs was evaluated. For this, we characterized the composition of gut microbiota of larvae reared in RAS and AS systems. Observed changes in gut MCs over time and between systems were highly correlated with shifts in water MCs. Differences in water physico-chemical properties corresponded with differences in water MC microbial composition. Mainly water MCs, and to a lesser degree feed MCs, were associated with changes in gut communities. Steering gut MCs could therefore be possible through water MC management tailored on the specifications of the rearing system in use.

The possibility of early life steering of gut communities via microbial manipulations of feed MCs was explored in chapter 4. We hypothesized that gut microbial composition is strongly shaped by selective pressures in the gut and by the MCs present in the water. Thus similar MCs should develop between treatments regardless the dietary treatments. 
Fish larvae were fed a control feed or the control feed containing MCs derived from aerobic, methanogenic or denitrifying sludge reactors. We found that gut microbiota shared a much higher number of operational taxonomic units (OTUs) with microbiota in sludge-based feeds than with water, resulting in distinct gut MCs between treatments. Our findings suggest that Nile tilapia gut $M C$ has a certain plasticity, which makes it amenable to interventions through proper feed microbial management.

In chapter $\mathbf{5}$ we tested the imprinting effect of early exposure to the probiotic Bacillus subtilis on shaping gut MC composition even after the administration of the probiotic discontinues. For this, we constrained the initial contact with microbes from the environment by producing axenic tilapia larvae. Subsequently, the imprinting hypothesis was tested by exposing the axenic larvae to normal husbandry conditions. $B$. subtilis was detected in the gut during the administration period. Early life probiotic exposure affected gut $\mathrm{MC}$ composition during $B$. subtilis administration but also after discontinuing administration of the probiotic. Although $B$. subtilis abundance dropped below the detection limit within 2 weeks after stopping administration, our findings indicated that the early exposure to the probiotic strain via the water had a sustained impact on gut MC composition.

Finally, in chapter 6 the main results of this thesis were discussed within the context of existing literature, and overall conclusions and practical implications for aquaculture production were presented. Additionally, a meta-analysis was performed with the dual purpose of examining (a) the phylogenetic similarity among gut MCs of the same and different fish species reared in different habitats, fed different diets and at different developmental stages and (b) the factors primarily shaping gut MCs. We showed that the selective pressure responsible in shaping gut MC composition highly depends on the host as gut communities clustered primarily together by host and to a lesser extend reflected differences in habitat and diet. The phylogenetic analysis of gut communities revealed a clear clustering by study indicating that manipulation of gut communities is conceivable. Study-to-study variation could be attributed to the methodology used for MC analysis highlighting also the importance of methodological uniformity when comparisons between studies are made.

Overall, this thesis provided fundamental knowledge on MC composition and development in aquaculture rearing systems. The insights generated are still too premature to fully explain, predict or steer MC composition. Additional studies are needed extending the experiments described here to other species and environments, executed following guidelines for optimization of experimental design and methodological conformity as suggested in this thesis. In the long run, this approach will facilitate the development of safe and effective methods for manipulating gut microbial composition to promote fish health in aquaculture rearing systems. 


\section{Acknowledgements}

This thesis marks the end of a long and important journey and there are many people that I would like to thank for their support along the way.

I would like to thank, first and foremost, my promotor, Johan Verreth. Dear Johan, thank you for giving me the privilege of conducting research in your group. I still remember every single question of yours during my first interview; both the serious and the teasing ones. It has been challenging, exciting and inspiring to work with you; from the beginning till the end. I am grateful for you being available when it was really necessary. You door was open, and though I was always asking for a minute, we ended up talking for one hour. I have learned a lot from you that will follow me in life. I have become better and stronger.

Dear Marc, thank you for your guidance, patience and endless support throughout my PhD project. Your encouragement kept me going. You provided me with the peace of mind in the times of stress. You spent a tremendous amount of hours on scientific discussions and many more. I will not forget our discussions about family, economy, politics, stoics, technology. I will always be thankful for your generosity, inspiration, and willingness to grant me more intellectual autonomy than I was ever entitled to.

Dear Detmer, I will always be grateful for having you in the project. Things could have been way different if you were not a part of the team. With you I realized that to the academic world, time is one of the most valuable resources, but there is still plenty of generosity therein. Thank you for the vibrant, collaborative and positive aura you brought in the project. Thank you also for your scientific contribution and for sharing with me your vision and passion for scientific discovery.

Dear Hauke, thank you for taking intense academic interest in this study and for providing valuable suggestions that improved the quality of this study. You have been there always caring to help. You are one of the busiest scientists I have ever met, but the fastest in responding; even after midnight. Thank you for generously giving me amounts of your time, energy and thoughts over the years. Thank you for your patience and composure when I was frustrated the most (and I bet it was often).

The AFI staff members, Johan S., Ep, Leon, Leo, Roel, Paul, Ronald O., Geertje, Tino, Ronald B., Tim, Arjen, Menno, Wian, Aart, Sietze, Betina, Sander, Truus. Thank you for the nice and collaborative atmosphere at work.

Gera, Linda, Annet thank you very much for helping me at every moment with any "bureaucratic" matter making the flow of my work and my time at AFI easier. Eugenia, thank you also for: "prioritizing", translating, booking, paying, making the Dutch calls, and for sharing in general. 
Special thank is for the students I supervised, Amin, Paulo, Catarina S., Hera, Papius, Pandu, Ivan, Bram, Joost. Thanks for your enthusiasm; you all contributed a piece to the work of this thesis.

A big thank for all the colleagues and friends I made among the AFI PhD fellows. Thank you Davood, Kim, Roel, Joost, Marit, Tu, Devi, Thomas, Happy, Goncalo, Miriam, Catarina M., Katharina, Grace, Desrina, Konrad, Stephan, Ilse for the in and out office hours we had together. Thank you for listening, for sharing your coffee and lunch breaks, for being upset and frustrated, for asking and giving advice. We were on the same boat, but here, I am getting out. I will miss you all!

I would like to express my heartiest thanks to all the colleagues from the Microbiology group (my second home). I am particularly indebted to Gianina and Hans, for all the exceptional molecular work, the long collaboration and for making me feel welcome every day in the lab. Javi, thank you for your help in bioinformatics and for always answering my endless questions; your contribution to my research has been fundamental.

In the following I would like to thank the Promicrobe colleagues, without which, this project would not have been possible. My dearest acknowledgments go to P. Bossier, P. Sorgeloos, and K. Dierckens from Gent University; J. Gatesoupe and S. Kaushik (for his scientific support and advice for life) from INRA; O. Vadstein, Y. Olsen, I. Bakke from NTNU; I.M. Aasen and J. Skjermo from SINTEF. Thank you all for the scientific input, the endless discussions and the possibility to study the amazing fish gut microbiome.

My gratitude goes to all people in the Aquaculture Research Institute, Hagerman Fish Culture Experimental Station, University of Idaho, USA. In particular I would like to thank R. Hardy and K. Overturf for giving me the opportunity to work together, for showing trust to my abilities and for contributing to my development as a scientist. Andreas, I would like to thank you for what you have done for me so far. It all started back in Athens during the M.Sc. applying for Ph.D. positions and dreaming. We had to be careful with the dreams because they come true sometimes, right? There we are! I am grateful for all the smoking brainstorming and for arguing over a cup of coffee (occasionally gin).

Julia, Saravanan, Vasco, Andre, Mahmoud, I met you at work and with time you have become friends for life, you have always been very supportive, you have been there with jokes and serious talks. You have made my life abroad a pleasure, no matter how tough a Ph.D., or life, has gotten at times. Thank you for all the coffees we shared outside AFI, for getting dizzy at conferences, for preparing our presentation the night before, for partying, for crying and for sharing personal things, for keeping still in contact, for being friends! I am grateful I have met you. Mahmoud, thank you for making the honor of being my paranymph, my colleague, my friend and for the priceless hours outside AFI. 
Pavlos and Manos, thank you for being my brothers. I am grateful to you for the four day and four country cycling tour (Nikos N. too) that we never managed to accomplish! Thank you for the dinners, the coffees, the endless talks (especially due to Manos), the arguments, the ups and the downs. Pavlos, without you we would not have succeeded in business, real estate, IT, BCT, farming and investments. Manos, without you I would not know about the modern history of Greece, politics, economics, financial crisis, CrossFit, Wikipedia. Thank you for the fail videos, the 80's advertisements, Nikolouli, the puzzles, MMA, the music nights with bad quality shampoo. Kostas K., Karolos and Stavros M. you have been indispensable to those too.

How not to mention my international and Dutch friends, Fabian, Mario, Ewout, Teo, Jodit, Diego, Edoardo, Teresa, Valentina, Hanna, Ale, lago, Derk, Jeroen, Stefano, thank you! We shared a lot throughout these years, dinners, drinks, fun and talks; you have become my family abroad. You guys have always been there to help and to make me feel home. Rafa, thank you for your friendship, your positive vibe and for the times you told me that it will be "alright". You know, you have someone to count on; just press the triangle!

Stavros N., Stavros K., Giannis, Kostas, Lambros, Makis, Nikos K., you have always been there and always be! Thank you for all the support you have been giving me despite the distance and the years that have passed since we last shared our youth.

I would also like to thank and apologize to all people that have somehow contributed to my thesis and, due to my terrible memory, are not included in these acknowledgements.

I would like to acknowledge all the sacrifices that my parents made to ensure that I had an excellent education. For this and much more, I am forever in their debt. Máva кaı патв́pa,

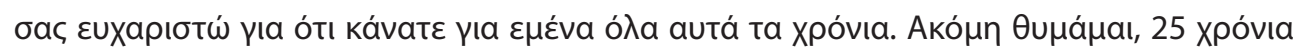

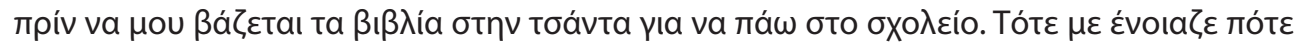

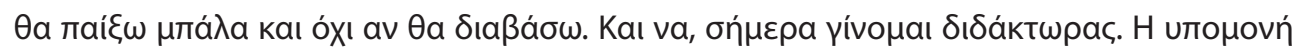

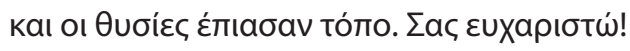

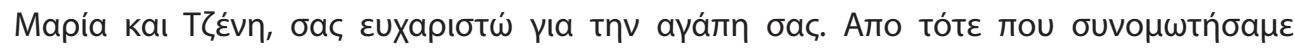

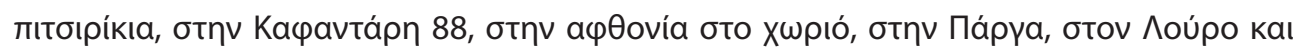

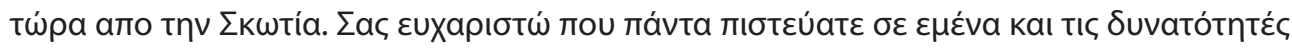

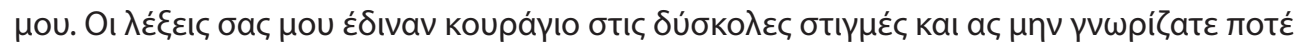

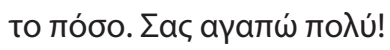

Lorenz and Allan, thank you for your moral support, for asking about my research and my life in the Netherlands; for visiting, for your hospitality, for your words of encouragement and for your love. Lorena and Jacob, your smiles were the oasis in the Ph.D. desert. Thank you for reminding me how carefree, warm and honest it feels to be child. 
Roberto, Paola, Chiara e Tea, grazie per avermi dimostrato come gli "in laws" possano essere una vera famiglia. Grazie per i vostri consigli e per il vostro sostegno. Sono grato per le vostre visite frequenti al Nord e la vostra ospitalità al Sud. Grazie per l'eccezionale cucina italiana (Roberto in particolare), l'alcol, il caffè normale (al mattino) e corretto (il resto del giorno) e per il letto a castello di Quercianella! Grazie a tutti per le lunghe chiaccherate intorno al tavolo (a cui partecipa anche Tea) e per la traduzione in inglese a seguire. Grazie per la vostra generosità e il vostro altruismo.

Beta, thank you for teaching me the meaning of trust, patience and hope. Thank you for finding fun in every little thing and encourage me to do the same. I don't thank you for the humiliation in streets, airports, cars, restaurant etc., but I still love you. I am grateful having you as a friend!

Giulia mia, where should I start from; how can I thank you for what you have done? Thank you for your love, your support, your devotion and your encouragement. Thank you for being you and standing always by my side! Thank you for being a rebel and being a stubborn one; I learned a lot from you. Thank you for arguing with me (even when you agree), for laughing with my bad jokes, for listening to Greek music on Sundays, for waking me up in the cold mornings, for proofreading my manuscripts, for occasionally cooking, for taking Beta out more than I do, for the "to do lists"... Without you, I wouldn't

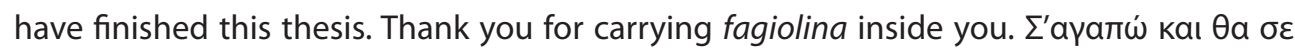
aүarí yıa návta! 


\section{About the author}

Christos Giatsis was born on August $13^{\text {th }}, 1981$ in Athens, Greece. After high school he started in 2001 his studies on Agricultural technology at the Technological Institute of Messolonghi, Greece. He graduated in 2005 completing a B.Sc. thesis on "Environmental state of Messolonghi lagoon complex" which was carried out at the Aquaculture and Fisheries department. In 2008 he obtained his M.Sc. degree from the department of Applied Hydrobiology of the Agricultural University of Athens with a thesis entitled "Partial replacement

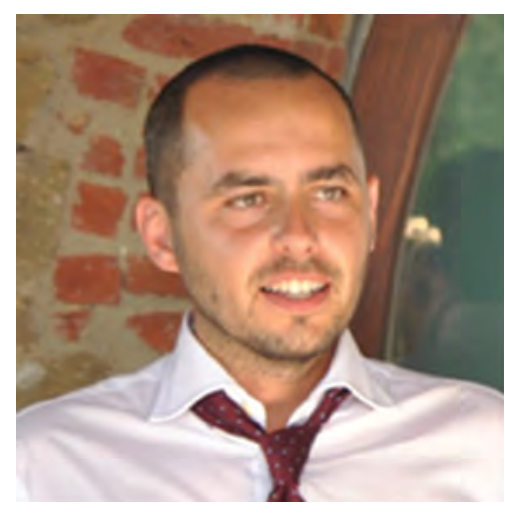
of commercial diet by housefly pupae in Rainbow trout fingerlings". In 2009 he started his Ph.D. research at the Aqauaculture and Fisheries group of Wageningen University as a part of the EU project "Promicrobe - Microbes as positive actors for more sustainable aquaculture". The results of his Ph.D. research are described in this thesis. In both 2013 and 2014 he collaborated as visiting scholar at Hagerman Fish Culture Experimental Station, University of Idaho, USA, working on "Examining the gut microbiome of rainbow trout fed plant protein diets". In the last year he has worked as a research assistant at the Aquaculture and Fisheries group of Wageningen University in a project entitled "Unlocking non-starch polysaccharides to improve feed utilization and probiotic benefits". He is currently employed as Project Head Probiotics of the Animal Nutrition and Care, by Evonik industries in Germany. 


\section{List of publications}

Giatsis C, Sipkema D, Smidt H, Verreth J, Verdegem M. (2014). The Colonization Dynamics of the Gut Microbiota in Tilapia Larvae. PLoS ONE 9: e103641.

Giatsis C, Sipkema D, Smidt H, Heilig H, Benvenuti G, Verreth J, et al. (2015). The impact of rearing environment on the development of gut microbiota in tilapia larvae. Scientific reports 5: 18206.

Giatsis C, Ramiro-Garcia J, Abernathy J, Verreth J, Smidt H, Sipkema D, Verdegem M (2016). Probiotic legacy on gut microbial assembly in fish larvae. Scientific reports (online).

Giatsis C, Verdegem M, Loo J van, Rohaan B, Verreth J, Smidt H, Ramiro-Garcia J, Sipkema D. (2016). Steering the gut microbiome of tilapia larvae with microbial diets (under review).

Giatsis C, Abernathy J, Loo J van, Borgos S. E, Degnes K.F, Aasen I.M, Untargeted metabolite profiling as a tool to understand the function of the gut microbiota in fish larvae (in preparation).

Ramiro-Garcia J, Hermes GDA, Giatsis C, Sipkema D, Zoetendal EG, Schaap PJ, et al. (2016). NG-Tax, a highly accurate and validated pipeline for analysis of 16S rRNA amplicons from complex biomes. F1000 Research 5: 1791.

Ramli Norulhuda M, Yusoff F.M, Giatsis C, Tan G.Y.A, Verreth J, Verdegem M. (2016). Inclusion effects of microalgae on water quality and bacterial community of recirculating aquaculture system (RAS) (submitted). 


\section{Training and Supervision Plan}

Education and training
The Basic Package
WIAS Introduction Course
Course on philosophy of science and/or ethics
Introduction interview with WIAS scientific director and secretary: $27 / 04$
Introduction interview with WIAS education coordinator: $16 / 04$
Introduction interview with WIAS PhD students confidant: $16 / 04$

Subtotal Basic Package

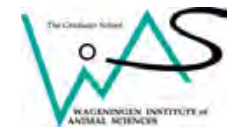

year

credits

2009

2010

2009

2009

2009

3.0

\section{Scientific Exposure}

year

credits

\section{International conferences}

Aquaculture Europe, Porto, Portugal, Oct 6-8

2010

Aquaculture Europe, Rhodes, Greece, Oct 18-21

2011

Aquaculture Europe, Prague, Czech Republic, Sept 1-5

2012

Aquaculture Europe, Rotterdam, Netherlands, Oct 20-23

2015

US Trout Farmers Association \& Idaho Aquaculture Association, Twin Falls, USA

2014

\section{Seminars and workshops}

Sole culture in the Netherlands, past, present and future, November $5 \quad 2010$

Resilience of fish in intensive systems, November 4

2010

\section{Presentations}

Oral presentation in Aquaculture Europe, Porto, Portugal, Oct 6-8

2010

Oral presentation in Aquaculture Europe, Rhodes, Greece, Oct 18-21

2011

Oral presentation in Prague, Czech Republic, Sept 1-5

2012

Oral presentation in Rotterdam, Netherlands, Oct 20-23

2015

2010

2011

WIAS Science Day-poster presentation

2012

WIAS Science Day-poster presentation

\section{In-Depth Studies}

year

credits

\section{Disciplinary and interdisciplinary courses}

Larvita training School, Algarve, Portugal, Nov 22-26

2010

Bionumerics-Applied maths course, Gent, Belgium Sept 5,6 \& 7

2011

Promicrobe course, Gent, Aug 20-22

2012

Technology for novel fish feeds, Olhao, Portugal, Oct 26-29

2014

Long term effects of diets low in fish oil and fish meal, Gran Canaria, Spain, Jan 13-15th

2016

\section{Advanced statistics courses}

Advanced Statistics course: Design of Experiments

\section{MSc level courses}

Aquaculture production systems (APS) 2009 


\begin{tabular}{|ll|}
\hline Statutory Courses & year credits \\
Use of Laboratory & 2009 \\
\hline
\end{tabular}

Subtotal Statutory Courses

3.0

\begin{tabular}{|ll}
\hline Professional Skills Support Courses & year \\
Teaching and Supervising Thesis Students & 2010 \\
Project and Time Management & 2011 \\
Techniques for Writing and Presenting a Scientific Paper & 2011 \\
Scientific Publishing & 2011 \\
Reviewing a scientific paper & 2011
\end{tabular}

Subtotal Professional Skills Support Courses

4.2

\begin{tabular}{|lc|}
\hline Research Skills Training & year \\
Preparing own PhD research proposal & 2009 \\
Advanced molecular techniques, University of Idaho, USA & 2014 \\
External training period & \\
Visiting scholar University of Idaho, USA & 2013 \\
\hline
\end{tabular}

Subtotal Research Skills Training $\quad 11.0$

\begin{tabular}{|lc|}
\hline Didactic Skills Training & year \\
Supervising practicals and excursions & \\
Research master cluster YAS 60312 supervision & 2010 \\
QAP course. Queens products excursion & 2011 \\
Supervising theses & 2009 \\
MSc Student (major) Amin Muhamad & 2010 \\
MSc Student (major) Paulo Mira Fernandes & 2010 \\
MSc Student (major) Catarina Silva & 2010 \\
MSc Student (major) Hera Suan & 2011 \\
MSc Student (major) Papius Tibihika & 2011 \\
MSc Student (major) Pandu Rizqi & 2012 \\
MSc Student (minor) Pandu Rizqi & 2012 \\
MSc Student (major) Ivan Tankowski & 2012 \\
MSc Student (major) Bram Rohaan & 2012 \\
MSc Student (major) Joost van Loo & \\
\hline
\end{tabular}

$\begin{array}{lr}\text { Subtotal Didactic Skills Training } & 20.1\end{array}$

\begin{tabular}{ll}
\hline Management Skills Training & year \\
Organisation of seminars and courses & credits \\
Membership of boards and committees & \\
Aquarius board member (EAS-SG Netherlands website administration) &
\end{tabular}

Subtotal Management Skills Training 3

Education and Training Total (minimum 30, maximum 60 credits) 

This study was carried out at the Aquaculture and Fisheries Group of Wageningen University, Wageningen, the Netherlands. The research described in this thesis was funded by The European Community's Seventh Framework Program (FP7/2007-2013) under grant agreement no. 227197 Promicrobe "Microbes as positive actors for more sustainable aquaculture".

Cover \& layout design : Iliana Boshoven-Gkini | AgileColor.com

Printed by: GVO drukkers \& vormgevers, Ede (NL) | gvo.nl 
